University of New Hampshire

University of New Hampshire Scholars' Repository

Spring 2020

\title{
Isospin dependence of Nucleon-Nucleon Short-range Correlations in Inclusive Scattering with Tritium and Helium-3
}

Shujie Li

University of New Hampshire, Durham

Follow this and additional works at: https://scholars.unh.edu/dissertation

\section{Recommended Citation}

$\mathrm{Li}$, Shujie, "Isospin dependence of Nucleon-Nucleon Short-range Correlations in Inclusive Scattering with Tritium and Helium-3" (2020). Doctoral Dissertations. 2522.

https://scholars.unh.edu/dissertation/2522

This Dissertation is brought to you for free and open access by the Student Scholarship at University of New Hampshire Scholars' Repository. It has been accepted for inclusion in Doctoral Dissertations by an authorized administrator of University of New Hampshire Scholars' Repository. For more information, please contact Scholarly.Communication@unh.edu. 


\title{
Isospin dependence of Nucleon-Nucleon Short-range Correlations in Inclusive Scattering with Tritium and Helium-3
}

\author{
BY \\ Shujie Li \\ B.S.,Sichuan University, 2012 \\ M.S.,University of New Hampshire, 2014 \\ DISSERTATION \\ Submitted to the University of New Hampshire \\ in partial fulfillment of \\ the requirements for the degree of
}

Doctor of Philosophy

in

Physics

May 2020 
This dissertation has been examined and approved in partial fulfillment of the requirements for the degree of Doctor of Philosophy in Physics by:

Dissertation director, Karl Slifer

Associate Professor of Physics

John Arrington

Senior Scientist at Argonne National Lab

Per Berglund

Professor of Physics

Maurik Holtrop

Professor of Physics

James Ryan

Professor of Physics

on May 14, 2020

Approval signatures are on file with the University of New Hampshire Graduate School. 


\section{Dedication}

For Patricia. 


\section{Acknowledgments}

The work demonstrated in this thesis is supported by the DOE Award DE-FG02-88ER40410 and DE-SC0014168. I would like to express my sincere gratitude to my Ph.D. advisors Patricia Solvignon and Karl Slifer for introducing me to the field of nuclear physics, and for their great support and guidance. I came to the University of New Hampshire through the graduate program of the Integrated and Applied Mathematics (IAM) in 2012. In 2014 I met Patricia when she came to UNH to give a seminar. That was also the first time I was exposed to JLAB physics. Patricia is the instructor of my nuclear physics class, and the spokesperson for my thesis experiment. In every aspect she is the most important person in my career so far. As advisors, Patricia and Karl are always friendly and supportive, I cannot be more grateful to have the opportunity to work with them.

I want to thank Dr. John Arrington and Douglas Higinbotham for both the physics they taught me and the support and care they offered to me. Thank my Master project advisor Kai Germaschewski, and my undergraduate project advisor Zhang, Shiqing for their guidance. Thank the IAM program for providing me the chance to explore both math and physics. Thank Professor Greg Chini, John Gibson, Mark Lyon, Jimmy Reader, Kevin Short, Dave Mattingly, John McHugh, Eberhard Moebius, Dawn Meredith, Karstern Pohl for their instructions. Thank Elena Long, Toby Badman, Ryan Zielinski, Nathaly Santiesteban, Leiqaa Kurbany, Bradley Yale from the UNH nuclear physics group for their friendship and support.

During my first four years of grad school I was lucky to met my friends and classmates, Jia 
Gao, Shan Wang, Dong Lin, Xiaoyan Chong, Feifei Wang, Gen Li, Liang Wang, Lei Wu, Minyi Chen, and many others. I still miss the time we discussed homework together, or went fishing, hiking, and BBQ together.

In 2016 I moved to Jefferson Lab to prepare for my thesis experiment, and met my fellow tritium students, Dien Nguyen, Tyler Hague, Jason Bane, Mike Nycz, Amanda Hoebel, Tong Su, Hanjie Liu, Bishnu Pandey, Hongxia Dai, Rey Cruz, Tyler Kutz, Sheren Alsalmi. We spent a lot of time in the testlab to clean HV cables, and also in the counting house staring at the beamtrip chart. We shared the anxiety of waiting, the excitement of data taking, and many important moments in our Ph.D. study and also our lives. Thank them for being in the great tritium experiment team with me and cover my back. During the tritium experiments I received a lot help from the postdocs in this project. They are Luke Meyers, Zhihong Ye, and Florian Hauenstein. Thank them for their excellent work on carrying the experiment forward.

I also need to thank students and scientists from the $G_{M}^{p}$ and DVCS collaborations, Longwu Ou, Barak Schmookler, Kalyan Allada, Eric Christy for sharing their experience with us. Thank the tritium experiments spokesperson for their excellent idea and their effort to make the project happen. Thank JLAB Hall A/C staff scientists and engineers, Ole Hansen, Bob Michael, Javier Gomez, Jessie Butler, Jack Segal, and many others for their support to this experiment. Thank Dave Meekins for the tritium target design and program management. During the time I spent at JLAB I also got a chance to work with the JLAB-CTEQ collaboration on the structure function F2 extraction. I learned a lot about both DIS experiments and QCD fittings from working with Alberto Accardi, Jeff Owen, Thia Keppel, Wally Melnitchouk, Nobuo Sato, Peter Monaghan, Ioana Niculescu, Gabriel Niculescu, Ishara Fernando, and Xiaoxian Jing. I met many friends at JLAB, including Danning Di, Shuang 
Han, Xinzhan Bai, Yan Huang, Yang Wang. Thank them for their friendship. And thank David Mack for taking me to kayaking.

Finally I want to thank my dissertation committee members Per Berglund, Maurik Holtrop, and James Ryan for their support and help during my thesis project. Thank my parents and husband for (not just) emotional support. Thank my cat for distracting me from starring at the computer screen. 


\section{Table of Contents}

Dedication. ....................................... ii

Acknowledgments................................ iv

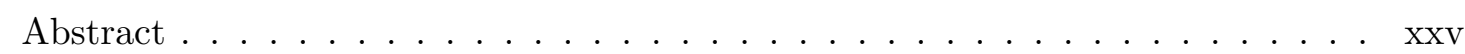

\begin{tabular}{lll}
\hline & Introduction & 1
\end{tabular}

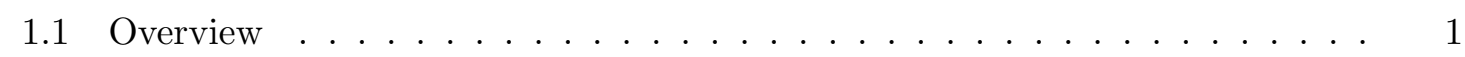

1.2 Nuclear Structure $\ldots \ldots \ldots \ldots \ldots \ldots \ldots$

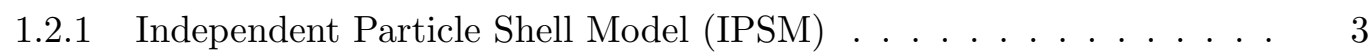

1.2 .2 NN Interactions $\ldots \ldots \ldots \ldots \ldots \ldots \ldots$

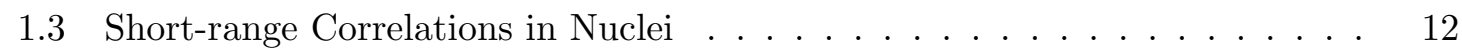

$1.3 .1 \quad$ Scaling variables for $\mathrm{QE}$ scattering $\ldots \ldots \ldots \ldots \ldots . \ldots \ldots$

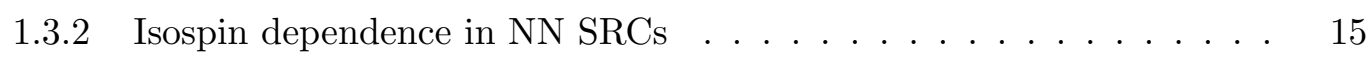

1.4 The E12-11-112 Experiment . . . . . . . . . . . . . . . . . . . . . . . . 19

$1.4 .1 \quad$ Physics Goals $\ldots \ldots \ldots \ldots \ldots$

1.4 .2 Connections to Other Experiments . . . . . . . . . . . 25

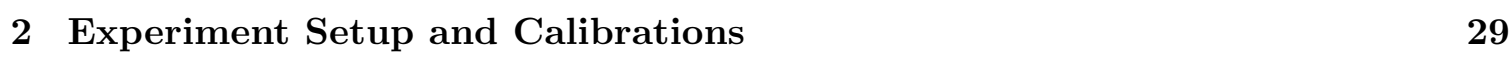

$2.1 \quad$ E12-11-112 Overview $\ldots \ldots \ldots \ldots \ldots \ldots$

2.2 Experiment site . . . . . . . . . . . . . . . . . . . . 30

2.3 Hall A Beamline . . . . . . . . . . . . . . . . . . . . . . . . . . . . . . 34

$2.3 .1 \quad$ Arc and Beam Energy Measurement . . . . . . . . . . . . . . . 35 
$2.3 .2 \quad$ Beam Current measurement . . . . . . . . . . . . . . . . . 36

$2.3 .3 \quad$ Beam Position $\ldots \ldots \ldots \ldots \ldots$. . . . . . . . . . . . . . . 38

2.4 The Target System . . . . . . . . . . . . . . . . . . . . . . . 42

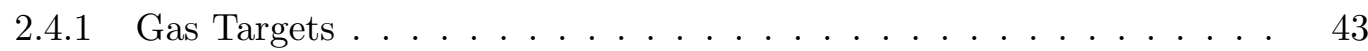

2.4 .2 Solid Targets $\ldots \ldots \ldots \ldots \ldots$. . . . . . . . . . . . . . 44

2.5 High Resolution Spectrometers $\ldots \ldots \ldots \ldots$. . . . . . . . . . . 45

2.6 Detector Package and Triggers $\ldots \ldots \ldots \ldots$

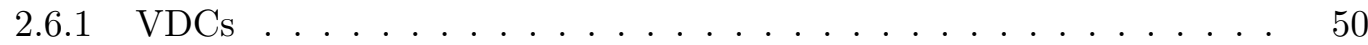

2.6 .2 Cherenkov . . . . . . . . . . . . . . . . . . 52

$2.6 .3 \quad$ Scintillators . . . . . . . . . . . . . . . . . . . . 54

2.6 .4 Pion Rejectors $\ldots \ldots \ldots \ldots \ldots \ldots$

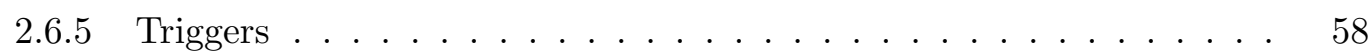

2.7 Data Acquisition $\ldots \ldots \ldots \ldots \ldots \ldots \ldots$

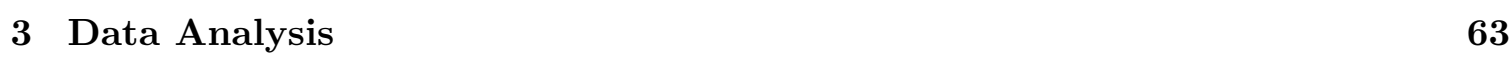

3.1 Hall A Analyzer Software . . . . . . . . . . . . . . . . . . . . . . . . 63

3.2 Event Reconstruction $\ldots \ldots \ldots \ldots \ldots$. . . . . . . . . . . . . 63

$3.2 .1 \quad$ Optics Calibration $\ldots \ldots \ldots \ldots$. . . . . . . . . . . . . . 64

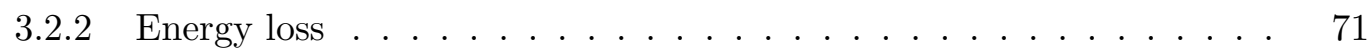

3.3 Event Selection and Efficiencies $\ldots \ldots \ldots \ldots$. . . . . . . . . . . . 75

$3.3 .1 \quad$ Beam Quality Check . . . . . . . . . . . . . . . . . . 75

3.3 .2 Trigger Efficiency $\ldots \ldots \ldots \ldots \ldots$. . . . . . . . . . . . . 77

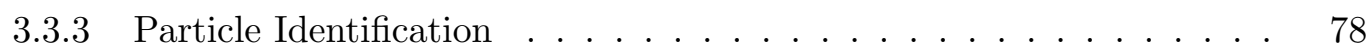

3.3 .4 Tracking Efficiency $\ldots \ldots \ldots \ldots$. . . . . . . . . . . . . . . . . . 83 


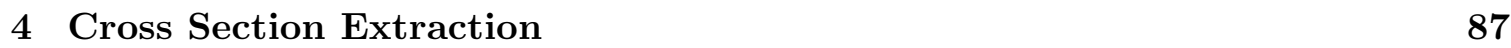

4.1 Yield Calculation . . . . . . . . . . . . . . . . . . . . . . . . . . . . 87

4.2 Gas Target Corrections . . . . . . . . . . . . . . . . . . . . . 90

$4.2 .1 \quad$ Endcap Contamination $\ldots \ldots \ldots$. . . . . . . . . . . . . 90

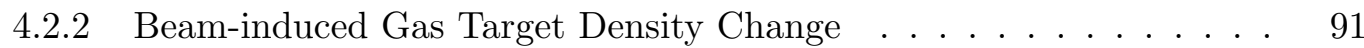

$4.2 .3 \quad$ Hydrogen Contamination in the Second Tritium Cell . . . . . . . . . 93

4.2 .4 Tritium Decay $\ldots \ldots \ldots$. . . . . . . . . . . . . . . 94

4.3 Monte-Carlo Simulation . . . . . . . . . . . . . . . . . . . . . . . 96

$4.3 .1 \quad$ Phase-space Generator . . . . . . . . . . . . . . . . . . . . . 96

$4.3 .2 \quad$ Cross Section Model . . . . . . . . . . . . . . . . . . . . . . . . . . . 97

$4.3 .3 \quad$ Data to Simulation Comparison $\ldots \ldots \ldots$. . . . . . . . . . 98

$4.3 .4 \quad$ Acceptance Cuts $\ldots \ldots \ldots \ldots$. . . . . . . . . . . . . . . . 999

4.3 .5 Binning $\ldots \ldots \ldots \ldots \ldots \ldots$

4.4 Absolute Cross Section Extraction . . . . . . . . . . . . . . . . . . . . . . 103

4.5 Cross Section Ratio Extraction $\ldots \ldots \ldots \ldots$

$\begin{array}{lll}5 & \text { Results and Discussions } & 109\end{array}$

5.1 Systematic Uncertainties . . . . . . . . . . . . . . . . . . . . . . 109

5.2 Absolute Cross Sections $\ldots \ldots \ldots$. . . . . . . . . . . . . . 112

$5.2 .1 \quad F(y)$ Fitting $\ldots \ldots \ldots \ldots \ldots \ldots \ldots$

5.3 Cross Section Ratios $\ldots \ldots \ldots \ldots$. . . . . . . . . . . . . 117

$5.3 .1 \quad$ NN-SRC Plateaus $\ldots \ldots \ldots \ldots$. . . . . . . . . . . 117

$5.3 .2 \quad$ The Isospin Preference in $\mathrm{A}=3$ Nuclei $\ldots \ldots \ldots$. . . . . . . . . . . 123

$5.3 .3 \quad$ Beyond $2 \mathrm{~N} \mathrm{SRCs} \ldots \ldots \ldots \ldots$ 
$\begin{array}{lll}6 & \text { Conclusions } & 130\end{array}$ 


\section{List of Tables}

2.1 List of kinematics. The first letter indicates L(eft) or R(ight) HRS. LS, PK, HS stand for lower momentum side, peak, and higher momentum side of the

QE peak. Momentum in $G e V / c$, quoted $Q^{2}$ in $\mathrm{GeV}^{2} / \mathrm{c}^{2}$. Those settings can be categorized into DIS region $(x<1)$, quasi-elastic region $(x=1)$, SRC region $(1<x<3)$, and $\mathrm{A}=3$ nuclei elastic region $(x=3)$. The beam energy and spectrometer angle calibration will be discussed in following sections. . 31

2.2 Energy correction factors for different beam passes from [34]. . . . . . . . 36

2.3 Target thickness from [46]. Note that the entrance window thickness of the 1st tritium cell is updated from 0.253 to 0.208 as suggested by Dave Meekins.

The 2nd tritium cell thickness shown here didn't include the hydrogen contamination (see section 4.2 .3 for details. $\ldots \ldots \ldots \ldots$

3.1 Carbon foil $\mathrm{z}$ position from L17 kinematics. . . . . . . . . . . . . . . 67

4.1 List of systematic uncertainties in the extracted yield. . . . . . . . . . . . 90

4.2 Tritium target density details. . . . . . . . . . . . . . . . . . 95

$4.3 \quad F(y)$ fitting parameters and other informations used in the XEMC cross section model. . . . . . . . . . . . . . . . . . . . . . 98

4.4 List of resolutions related to $x_{b j}$. The $y_{t g}$ resolution is from optics calibration. Angle and momentum resolutions are from $\mid 2-19] \ldots \ldots$. . . . . . . . 102

$5.1 \quad F(y)$ fitting results from extracted cross sections at L17 and EP. . . . . 115 
5.2 The heights of tritium (helium-3) to deuterium, as well as tritium to helium-3 plateaus fitted with L17 and EP data. Fits were performed with different ranges of $x_{b j}$ for the stability check. Fluctuations of the result are included in the fitting uncertainties. 


\section{List of Figures}

1-1 The lowest two energy shells in nuclei with odd mass number, Fig.17.6 from [6]. 4

1-2 PWIA diagram of QE electron scattering off a nucleus $A$. . . . . . . . 5

1-3 $\quad$ Momentum distributions from the $\left(\mathrm{e}, \mathrm{e}^{\prime} \mathrm{p}\right)$ measurement of the valence shell

(upper marks) and the next deeper shell (lower marks). Curves from Con-

tinuum Distorted Wave Impulse Approximation (CDWIA) calculations with

Woods-Saxon bound-state wave functions are scaled to fit the data. Figure

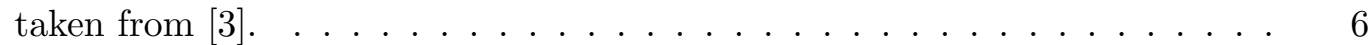

1-4 Spectroscopic strength for valence orbitals (left) and for states just above the

Fermi level (right) as measured at NIKHEF-K [3]. . . . . . . . . . . . 8

1-5 Nucleon momentum distributions from experimental data (symbols), shell model calculation (dotted line), and calculation including short-range correlation (solid line) $[2] .1 \mathrm{fm}^{-1} \approx 200 \mathrm{MeV} / \mathrm{c} . \ldots \ldots \ldots$

1-6 Three examples of modern NN potentials in the ${ }^{1} S_{0}$ (spin singlet and s-wave) channel: CD-Bonn [7], Reid93 [8], and AV18 [9]. Figure taken from [10]. . . 10

1-7 The NN potential calculated from AV18 [11]. . . . . . . . . . . . . . . . 10

1-8 The momentum distributions of $n p$ (lines) and $p p$ (symbols) pairs with relative momentum $q$ and total momentum $Q=0[12] \ldots \ldots \ldots . \ldots 11$ 
1-9 $\quad F(y)$ extracted from ${ }^{3} \mathrm{He}$ cross sections. Data are taken from SLAC and JLAB. The inset shows $F(y)$ at $0.7>y>1.1$ which corresponds to the region of interest for 3N-SRCs. For purely QE scattering $F(y)$ is symmetric about $y=0$. Data on the right side of the peak show deviations due to inelastic contributions. Figure taken from [16]. . . . . . . . . . . . . 13

1-10 Schematic drawing of $\left(\mathrm{e}, \mathrm{e}^{\prime}\right)$ total cross section as a function of energy transfer.

With fixed beam energy $x_{b j}$ decreases with $\omega \cdot \omega=Q^{2} / 2 m$ is the QE peak.

Figure taken from $[17] . \ldots \ldots \ldots$. . . . . . . . . . . . . 14

1-11 Feynman diagram of electron scattering on the SRC pair. Figure 17 from [18]. 15

$1-12$ Illustratiion of $\mathrm{C} 12\left(\mathrm{e}, \mathrm{e}^{\prime} \mathrm{pN}\right)$ reaction. [4] . . . . . . . . . . . 16

1-13 $n n, n p$, and $p p$ pair fractions in ${ }^{12} \mathrm{C}$ from JLAB E01-015 experiment [4. . . 16

1-14 the angle between the initial momentum of the scattered proton and the observed momentum of its paired neutron $\mathbf{p}_{n}$ as a function of the neutron momentum. Markers indicate data with different beam energies. . . . . . . 17

1-15 The relation between $p_{m} i n$ and $x_{b j}$ in electron scattering from ${ }^{2} \mathrm{H}$ (left) and $\mathrm{Au}$ (right) at $Q^{2}=0.5,1.5,3$, and $10 \mathrm{GeV} / \mathrm{c}^{2} \cdot p_{\min }$ is the minimum parallel momentum of the initial nucleon. Red lines indicate the Fermi momentum. Figure taken from $[18] . \ldots \ldots \ldots . \ldots \ldots$

1-16 The per-nucleon cross section ratios of various nuclei to deuterium from JLAB E02-019 experiment [20]. In general the ratios are flat between $1.4<x_{b j}<$ 1.8 in light nuclei. In heavy nuclei the smearing of the central momentum of the SRC pair is more complicated such that a direct observation of plateau is difficult, But $a_{2}(A)$ can still be extracted from the cross section ratio to provide information on the isospin dependence. . . . . . . . . . . . . . . 20 
1-17 The ${ }^{4} \mathrm{He}$ to ${ }^{2} \mathrm{H}$ cross section ratio with respect to $x_{b j}$ at $Q^{2}=0.9-2.3 \mathrm{GeV}^{2} / c^{2}[21]$. The vertical dashed line on each panel correspond to a final state that is 50 MeV greater than the deuteron rest mass. Data were taken at SLAC [22].

1-18 Cross section ratios of heavy nuclei over helium-3 from previous inclusive measurements. Results are inconsistent at $x_{b j}>2$. [11] . . . . . . . . . 24

1-19 Illustration of two extreme case of possible 3N SRCs configurations. [23] . . 25

1-20 Per-nucleon cross section ratios of ${ }^{48} \mathrm{Ca} /{ }^{40} \mathrm{Ca}$ from experiment E08-14 [25]. 26

1-21 Nuclei to deuterium per-nucleon cross section ratios. Figure taken from [26] 27

1-22 Top: Illustration of SRC and EMC ratios from [26]. Botom: A linear fit of EMC ratio versus $a_{2}(A)$ from $[29] . \ldots \ldots \ldots \ldots . \ldots \ldots$

2-1 Kinematics coverage of the E12-11-112 experiment. . . . . . . . . . . 32

2-2 $\quad$ Schematic of CEBAF after the $12 \mathrm{GeV}$ upgrade. Figure 1 from [31]. . . . . 33

$2-3$ Side view of Hall A. Figure 2 from $[32] . \ldots \ldots \ldots \ldots$

2-4 Schematic drawing of Hall A during E12-11-112 experiment. Adapted from [33].

$2-5 \quad$ Drawing of the arc section of the Hall A beam line from [35]. . . . . . . . . 35

$2-6 \quad$ Schematics of beam current instruments from Ref. [37]. . . . . . . . . . 37

2-7 A linear fit of UNSER frequency against the wire current, and the residual test. Figure taken from $[39] . \ldots \ldots \ldots \ldots$. . . . . . . . . . . 38

2-8 Linear fit of the UNSER-measured beam current against the BCM dnew frequency from $[40] . \ldots \ldots \ldots \ldots$

2-9 $\quad$ BPMs and Harps position in Hall Coordinate System. Figure 3.7 from [35] 39

2-10 Diagrams of the BPM chamber from [43]. . . . . . . . . . . 40

$2-11$ The harp scanner. [43]. . . . . . . . . . . . . . . . 40 
2-12 Beam positions read from the calibrated BPMs comparing to Harp scan results 42

2-13 The reconstructed reaction vertex distribution on the target plane with the carbon hole target. The hole in the center has a known diameter of $2 \mathrm{~mm}$. The ratio between the fitted diameter from the plot and the actual hole diameter provides a scaling factor for the raster calibration. Figure taken from [4]. . . . . . . . . . . . . . . . . . . . . . . . . . . . . . . . . . 42 42

2-14 A linear fit of the raster current with respect to the reconstructed vertex $z$ position. The slope is 0 within uncertainties, which indicates no correlation between the raster current and the reconstructed vertex $\mathrm{z}$ position, as it supposed to be. Figure taken from [44]

2-15 Phase lag between the BPM-measured beam position and the raster current. 43

2-16 A diagram of target ladder from the target control GUI. . . . . . . . . . . . 44

$2-17$ An engineer drawing of target design details. . . . . . . . . . . . . . . . . . 45

2-18 HRS layout. Figure 5 from [32]. . . . . . . . . . . . . . . . . . . . . . . . . 47

2-19 HRS design characteristics. Table 1 from [32]. . . . . . . . . . . . . . . . . 48

2-20 LHRS acceptance from the Hall A Monte-Carlo simulation (see Sec. 4.3) as

a function of in-plane angle $\left(-0.04 \mathrm{rad}<\phi_{t g}<0.04 \mathrm{rad}\right)$ and out-of-plane angle $\left(-0.06 \mathrm{rad}<\theta_{t g}<0.06 \mathrm{rad}\right)$ at L17-PK. Each subplot covers $1 \%$ of $\delta_{t g}$ range. ........................... . . . 49

2-21 Schematic drawing of Left HRS detector package. Letters and numbers correspond to each PMT signal as is indexed in Analyzer. . . . . . . . . . . . . 50

$2-22$ Schematic configuration of VDCs from [50] . . . . . . . . . . . . . . . . . . 51

2-23 A typical 5-cell track event. . . . . . . . . . . . . . . . . . . 52 
$2-24$ Time spectrum of a VDC wire from figure 17 in [50]. . . . . . . . . . 53

2-25 3D and open view of Cherenkov from Ref. [52]. 10 curved mirrors on the

back of Cherenkov chamber is used to redirect Cherenkov light to 10 PMTs (five on each side). $\ldots \ldots \ldots \ldots \ldots \ldots \ldots$

2-26 Tracks of events fired each PMT projected to the Cherenkov plane. . . . . 55

$2-27$ The Cherenkov PMT raw ADC spectrum. . . . . . . . . . . . . . . 56

$2-28$ The Cherenkov PMT ADC spectrum after calibration. . . . . . . . . . 57

$2-29$ Schematic drawing of LHRS pion rejectors from [53]. . . . . . . . . . 58

2-30 The sum of electron energy deposit in two layers of pion rejectors divided the track momentum is plotted with respect to the vertical projection of the track on the detector plane. . . . . . . . . . . . . . . . . . 58

2-31 ADC singal amplitude from S2, PRL1, and PRL2 PMTs are plotted at each detector plane. Star Marker: the particle hit position estimated from ADC signals. Red Marker: the track projection on each detector plane. . . . . . . 59

$2-32$ Schematics of LHRS triggers design by Florian Hauenstein. . . . . . . . . . 62

3-1 Schematics of LHRS misspointing information provided by the encoder. . . $\quad 66$

3-2 Code to calculate LHRS angle and offset from encoder and lvdt signals at each start of run on adaq computer (filename: tritium_db_L.C). The angle and offset are written into run database and used by Analyzer to reconstruct reaction point. Note that by convention the offset is positive when the spectrometer is pointing upstream. However during this experiment run period the variable HacL_CalcPoint is recorded in the opposite way. . . . . . . . . 66 
3-3 Schematic of LHRS pointing. Grey dashed line is the nominal central ray. orange solid line is the actual electron track. The setting as shown has a positive HRS offset since the central ray is pointing to the upstream of hall center, and the target position is negative. . . . . . . . . . . . 68

3-4 The reconstructed vertex $\mathrm{z}$ position of the multifoil target. The $25 \mathrm{~cm}$ long target consists 11 carbon foils evenly spaced. But in the target installation for the 2018 fall run period one foil was damaged. So we see only 10 peaks

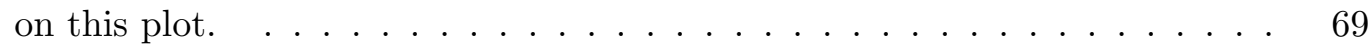

3-5 The optimized $y_{t g}$ of each foil (red) comparing to the nominal foil position (green) for L17-SRC2. . . . . . . . . . . . . . . . . . 70

3-6 Left: The sieve is installed at the entrance of RHRS. Right: A drawing of sieve design. . . . . . . . . . . . . . . . . . 71

3-7 Sieve pattern of the central foil before (left) and after (right) $\phi_{t g}$ calibration for L17-SRC2 kinematics. . . . . . . . . . . . . . . . . . . . . . . 71

3-8 Hydrogen elastic calibration run 111702 at 13.2 degree during $\mathrm{e}^{\prime} \mathrm{K}$ experiment, momentum was set at $3.93 \mathrm{GeV}$ to match the L17-SRC2 kinematics.

Plots show hydrogen invariant mass with respect to the out-of-plane angle $\theta_{t g}$ before (top) and after (bottom) manually tuning D100, D200 terms. After calibration the invariant mass becomes uncorrelated with $\theta_{t g} . \ldots . . .72$

3-9 The hydrogen invariant mass from L17-PK (top) and L17-SRC1 (bottom) after calibration . . . . . . . . . . . . . . . . . 73

3-10 Schematic of incident and scattered electron energy loss [60] . . . . . . . 74 
3-11 The energy loss rate of $500 \mathrm{MeV}$ pion goes through silicon. Figure taken from figure 32.8 in[61] to show that the most probable energy loss is a better approximation to the struggling function comparing with the mean energy

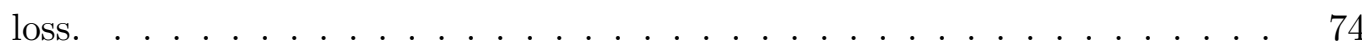

3-12 Flow chart on selecting events with stable beam. . . . . . . . . . . . 76

3-13 Example of event selection with run 3605. Top: the 1-d spectrum of beam current. Bottom: a zoomed-in plot of beam current versus time to show how the beamtrips were excluded. Events that fall into $\pm 1.5 \mu \mathrm{A}$ of mean current (between two dashed line, with red markers) are the events that were used

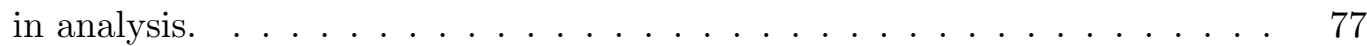

3-14 Trigger type distribution from run 100684. The $\mathrm{x}$ axis is the binary sum of trigger type. For example, an event with only T3 fired would have evtypebits $=2^{3}=8$. More than $99 \%$ of electron events fired all three triggers. $\ldots \ldots$. . 79

3-15 Distributions of T3-only events on Q3 exit aperture and the S0 plane, and the corresponding $\mathrm{S} 2$ signals. $\ldots \ldots \ldots$. . . . . . . . . . . . 79

3-16 The Cherenkov signal versus $\mathrm{E} / \mathrm{P}$ ratio after calibration. . . . . . . . . . . 80 3-17 The peak of Cherenkov sum signal was fitted to a Poisson distribution. . . . 81 3-18 Upper: the first versus second layer of pion rejector signals before (left) and after (right) Cherenkov cut. Lower: the ratio of pion rejector sum signal to the track momentum. The red line indicates a linear extrapolation of the electron distribution to $E / P<0.7$. The blue peak at $E / P \nmid 0.1$ are mostly pions 82 
3-19 Left: distribution of no-track events with respect to cluster numbers. Right: TDC signals from U2 plane of a no-track event with three clusters. The algorithm failed to identify the "V" shape due to the unexpected rise on the

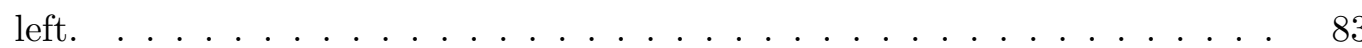

3-20 Total number of clusters from four VDC planes. Shaded area represents all events after PID cuts. No track events in red, one track events in blue, and multi-track events in purple. $\ldots \ldots \ldots$. . . . . . . . . . . 84

3-21 The amount of multi-track events with loose acceptance cuts and vertex $\mathrm{z}$ cut is less than $0.4 \%$ of all good electron events. . . . . . . . . . . 84

3-22 The percentage of no-track events versus the U1 plane. In the Spring 2018 run period the wire \#240 on U1 plane was noisy, which caused an inefficiency spike. This wire was fixed before the fall 2018 run period. This effect was demonstrated to be cancelled in the ratio analysis. . . . . . . . . . . 85

3-23 All good electron events (blue) and good electron events with U1 wire \#240 fired (red) are plotted with respect to momentum (top) and $x_{b j}$ (bottom). . $\quad 86$

4-1 Normalized yield, efficiencies, livetime, and beam current for every L17-SRC2 run with Helium-3 target. . . . . . . . . . . . . . . . . . . . . . . 89

4-2 Gaussian fits of entrance (top) and exit (bottom) window yield distributions \begin{tabular}{|c|c|c|}
\hline along vertex $z$ for various targets at L17-PK kinematics. Note that the yield \\
\hline
\end{tabular} is normalized by charge and also the quoted window thickness. Though a significant difference in amplitude is shown between the exit windows of the empty cell and dummy target, their integrals between $-8 \mathrm{~cm}<z<0$ agree

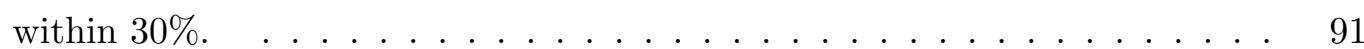


4-3 Helium-3 yield with respect to $x_{b j}$ from four overlapped kinematics at 17 degrees. The lower group of markers are the total endcap contributions. Dashed lines are Monte-Carlo simulations, see Sec.|4.3| for details. . . . . . 92

4-4 Yield distributions from gas targets and empty cell along vertex $z$ at L17-PK. Numbers shown on the legend are the integrated yield between $-8 \mathrm{~cm}<z<8$ cm.

4-5 Subtracting the hydrogen contamination peak with real hydrogen elastic data at the same kinematics. . . . . . . . . . . . . . . . . . . 94

4-6 Data to Monte-Carlo simulation yield comparison with the carbon foil at L17PK kinematics with loose acceptance cuts. The integrated data to MonteCarlo yield ratio is $1.034 . \quad \ldots \ldots \ldots \ldots$

4-7 Data to Monte-Carlo simulation yield comparison with helium-3 target at L17-PK kinematics with loose acceptance cuts and vertex $z$ cut. The inte-

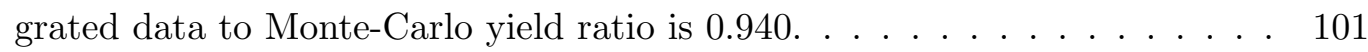

4-8 Data to Monte-Carlo simulation yield comparison with helium-3 target at L17-PK kinematics with tight acceptance cuts and vertex $z$ cut. The inte-

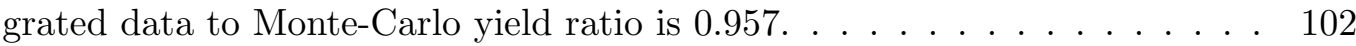


4-9 Top left: Data to Monte-Carlo simulation yield comparison with helium3 target at four overlapped L17 kinematics with respect to the scattered electron momentum $E^{\prime}$. Dashed lines represent yield from simulation. Lower markers are contributions from endcaps. Bottom Left: data to simulation ratio. Top (bottom) right: bin-by-bin endcap contamination from the target entrance (exit) window with respect to $E^{\prime}$. The discrepancy between L17SRC1 and L17-SRC2 entrance window contribution was absorbed into the $30 \%$ uncertainty in the endcap subtraction. . . . . . . . . . . . . . 103

4-10 Data to Monte-Carlo simulation yield comparison, and endcap contamination of helium-3 target at four overlapped L17 kinematics with respect to $x_{b j} . \quad$. 104

4-11 2D Event distribution in scattering angle and momentum. Red box: tight acceptance cuts. . . . . . . . . . . . . . . . 105

4-12 $x_{b j}$ resolution for L17 kinematics. Other settings have similar or higher res-

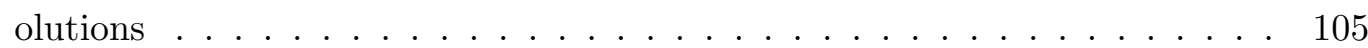

4-13 Data to Monte-Carlo simulation yield comparison of Carbon foil at L17-PK kinematics with tight acceptance cuts. The simulation is scaled by a factor of 1.05 to check the shapes of distributions. . . . . . . . . . . 106

4-14 Compare the angle(left) and $Q^{2}$ (right) distribution within a given $x_{b j}$ bin.

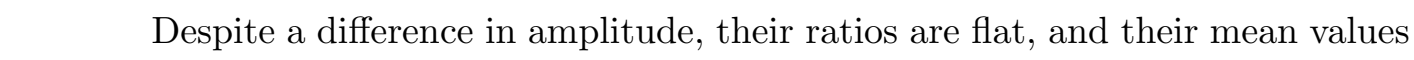
are consistent within resolution. . . . . . . . . . . . . . . . . 107

4-15 Top: the Born to radiative cross section ratio with tritium and helium-3 \begin{tabular}{|c|c|}
\hline target. Bottom: the radiative correction factors from two targets agree within \\
\hline
\end{tabular}

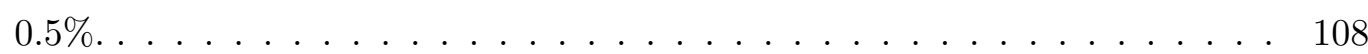


$5-2{ }^{3} \mathrm{H} /{ }^{3} \mathrm{He}$ raw event counts ratio with different acceptance cuts and there difference in percentage with respect to the loose cuts(red) from kinematics L17-SRC1(left) and EP-SRC(right). . . . . . . . . . . . . . . . . 111

5-3 Extracted Deuterium nucleus Born cross sections. The error bars represent all point-to-point uncertainties (statistical and systematic) added in quadrature. Comparing to L17 (green), the L30 data (blue) has lower $Q^{2}$ hence smaller inelastic contamination but larger QE contribution. The combination of those two effects happen to create similar total cross sections for L17 and L30 at the $x_{b j}=1$ QE peak. . . . . . . . . . . . . . . 113

5-4 Extracted Tritium Born cross sections from various kinematics. . . . . . . . . 114

5-5 Extracted Helium-3 Born cross sections from various kinematics. . . . . . . 114

5-6 Left: extracted deuterium $F(y)$ from this experiment. Right: the high $Q^{2}$ data (L17 and EP) are used for new $F(y)$ fitting. . . . . . . . . . . . 116

5-7 Left: extracted tritium $F(y)$ from this experiment. Right: the high $Q^{2}$ data (L17 and EP) are used for new $F(y)$ fitting. $\ldots \ldots \ldots \ldots$

5-8 Left: extracted helium-3 $F(y)$ from this experiment. Right: the high $Q^{2}$ data (L17 and EP) are used for new $F(y)$ fitting. . . . . . . . . . . . . . 117

5-9 Deuterium yield (not normalized) from L21-SRC in log scale. The bump at $x_{b j}=2$ corresponds to deuterium elastic scattering. The red curve is the sum of a Landau distribution and a mirror-inverted Landau distribution. The blue curve is the fitted elastic peak. . . . . . . . . . . . . . . . . . 118 
5-10 Tritium to deuterium (top), and helium-3 to deuterium (bottom) cross section ratios with respect to $x_{b j}$ from various kinematics settings. $Q^{2}$ is quoted at the QE peak. The double error bars represent the statistical uncertainties and the sum of statistical and systematic point-to-point uncertainties. . . . 119

5-11 Fit the 2N-SRC plateaus with tritium to deuterium (top), and helium-3 to deuterium (bottom) cross section ratios from L17 and EP. . . . . . . . . 121

5-12 Tritium to deuterium (top), and helium-3 to deuterium (bottom) cross section ratios with respect to $\alpha_{2 N} . \ldots \ldots \ldots \ldots \ldots$

5-13 Tritium to helium-3 cross section ratio with respect to $x_{b j}$ from various kinematics setting. . . . . . . . . . . . . . . . . . . . . 124

5-14 Fit the 2N-SRC plateaus with helium-3 to tritium cross section ratios from L17 and EP.

5-15 $n p$ to $p p$ SRC pair ratio extracted from the inclusive ${ }^{3} \mathrm{H} /{ }^{3} \mathrm{He}$ cross section ratio in this thesis (top), and the exclusive $e^{\prime} p$ experiment [28] (bottom). . . 126

5-16 The nucleon momentum distribution between nucleon-nucleon pairs as a function of the pair's relative momentum $q=k_{12}=(k 1-k 2) / 2$. The distribution is projected into $n n$ and $n p$ pairs (left) and spin-isospin (ST) configurations (right). The figure on the right also gives the density integral of each configuration. Figures taken from $[74]$. 


\author{
ABSTRACT \\ Isospin dependence of Nucleon-Nucleon Short-range Correlations in \\ Inclusive Scattering with Tritium and Helium-3 \\ by \\ Shujie Li \\ University of New Hampshire
}

The nucleon-nucleon (NN) potential has a strong repulsive core. When a two-nucleon (sub)system falls into this range, they will interact strongly at short distance, and move away from each other with momenta above the Fermi level. This is called the NN Shortrange Correlations (SRCs). Previous experiments reported a neutron-proton pair (isosinglet) dominance in high-momentum nucleons. In inclusive electron scattering, this $n p$ dominance will cause a scaling behavior of cross sections at $1.4<x_{b j}=Q^{2} / 2 m \omega<2$ where the high-momentum nucleons dominate. At Jefferson Lab Hall A we checked this hypothesis with electron Quasi-elastic (QE) scattering on $A=3$ nuclei systems. Our unique gas cell design made electron-tritium scattering possible for the first time in a decade. The ${ }^{2} \mathrm{H},{ }^{3} \mathrm{H}$, and ${ }^{3} \mathrm{He}$ inclusive cross sections at $1<x_{b j}<3$ were measured in two experiments (E12-11-112 and E12-14-011) with a wide $Q^{2}$ range $\left(0.4<Q^{2}<3 \mathrm{GeV}^{2}\right)$. This thesis discusses the physics goals, experiment setup, and data analysis of the E12-11-112 experiment. Ratios of ${ }^{3} \mathrm{H} /{ }^{2} \mathrm{H},{ }^{3} \mathrm{He} /{ }^{2} \mathrm{H}$ and ${ }^{3} \mathrm{H} /{ }^{3} \mathrm{He}$ as well as absolute cross sections are presented at $0.4<Q^{2}<1.8 \mathrm{GeV}^{2}$. NN SRC plateaus are observed in the cross section ratios at high $Q^{2}$. The ${ }^{3} \mathrm{H} /{ }^{3} \mathrm{He}$ ratio of $0.84 \pm 0.01$ at NN SRC dominant kinematics indicates a $n p$ to $p p$ SRC pair ratio of $2.2 \pm 0.2$ in $\mathrm{A}=3$ nuclei. That implies a preference in $T=0$ isospin singlet configuration. No plateaus are observed at $x_{b j}>2$. 


\section{Chapter 1}

\section{Introduction}

\subsection{Overview}

In 1911, Ernst Rutherford discovered the small dense and positively charged nucleus inside the atom by scattering $\alpha$ particles off thin gold foils. Later experiments revealed that the nucleus consists of positively-charged protons and charge-neutral neutrons with almost the same mass. Werner Heisenberg and other scientists applied the new formality of quantum mechanics to explain the nucleus as a collection of almost identical nucleons (protons and neutrons) bond by the strong nuclear force at short distance. Though the origin of such a nuclear force was not known, the system of nucleons could be described with many-body Schrödinger equations as wave functions that are subject to inter-nucleon potentials. This framework is still the base of nuclear physics today. After the discovery of quarks in the 1970s, Quantum-chromo-dynamics (QCD) was developed to describe the strong interaction of quarks and gluons inside the nucleons, and people began to study how nuclear forces emerged from quark-level interactions.

The phenomenological free nucleon-nucleon $(\mathrm{NN})$ potential is weakly attractive at long distances, strongly attractive at intermediate distances, and has a hard repulsive core. The attractive force at long-to-intermediate distances is responsible for the nuclear bound state. And the repulsive core prevents a nucleus from collapsing. In low energy nuclear 
physics, the nucleon interactions are usually described by the exchange of mesons, which is fairly successful in explaining interactions at low momentum transfer and matches the QCD approach qualitatively. However, it could not describe nucleon interactions at short distance where the pion-nucleon distance is comparable to the nucleon size of $\sim 0.8 \mathrm{fm}$. The meson exchange models are also not in agreement with some basic QCD predictions [1]. Early calculations showed that the nucleon-nucleon interaction at short distance plays an important role in generating the high momentum component in the nucleon momentum distribution [2]. Probabilities of finding nucleons above the Fermi level is as large as $35 \%$ in medium and heavier nuclei [3]. Those high momentum nucleons can be identified through $\mathrm{A}\left(\mathrm{e}, \mathrm{e}^{\prime}\right) \mathrm{X}$ experiments. It has been shown in exclusive scattering experiments that a high momentum nucleon knocked out from the nucleus is always observed in coincidence with another high momentum nucleon emitted with an almost opposite initial momentum. Those correlated nucleons are dominated by proton-neutron pairs [4]. This indicates a strong isospin preference in the nucleon-nucleon short-range correlations (NN SRCs) due to the presence of the tensor force in the isospin singlet state.

The experiment E12-11-112 at Jefferson Lab (JLAB) is designed to investigate the isospin dependence of NN SRCs in inclusive electron scattering. Cross sections from mirror nuclei ${ }^{3} \mathrm{H}$ and ${ }^{3} \mathrm{He}$ were measured at $0.7<x_{b j}=Q^{2} / 2 m \omega<3$ (See Sec. 1.3.1). In the range of $1.4<x_{b j}<2$, the scaling behavior from NN SRCs leads to an asymptotic plateau in the ${ }^{3} \mathrm{H}$ to ${ }^{3} \mathrm{He}$ cross section ratio. In other words, the ratio goes to a constant value. A precise measurement of the plateau height would reveal the isospin preference in the $A=3$ nuclei system. The $x_{b j}>2$ cross section ratios are expected to provide information on momentum sharing configurations in the possible 3N SRCs. 


\section{$1.2 \quad$ Nuclear Structure}

\subsubsection{Independent Particle Shell Model (IPSM)}

Assuming that nucleons are structure-less particles, the dynamics of a nuclear system with A nucleons could be described by the non-relativistic schrödinger equation:

$$
\left[\sum_{i}-\frac{\hbar^{2}}{2 M_{N}} \nabla_{i}^{2}+\sum_{i<j} v_{2}\left(\mathbf{x}_{i}, \mathbf{x}_{j}\right)+\sum_{i<j<k} v_{3}\left(\mathbf{x}_{i}, \mathbf{x}_{j}, \mathbf{x}_{k}\right)+\ldots\right] \Psi_{A}=E_{A} \Psi_{A}
$$

where $\Psi_{A}\left(\mathbf{x}_{\mathbf{1}}, \mathbf{x}_{\mathbf{2}}, \ldots\right)$ is the nuclear wave function and $v_{2(3)}$ is the two (three)-nucleon potential. Knowledge of the Hamiltonian and analytical or numerical solvers are required to understand this nuclear system. The Quantum Monte-Carlo calculations (QMC) of light nuclei and nuclear matter is possible with realistic nuclear Hamiltonians that fit nucleonnucleon scattering data [5]. For heavy nuclei, a classic recipe is provided by the mean field theory, which assumes that nucleons move independently in a summed potential $U(x)$ of all nucleon interactions. With this zeroth-order approximation, Eq. 1.1 reduces to

$$
\left[-\frac{\hbar^{2}}{2 M_{N}} \nabla_{i}^{2}+U\left(\mathbf{x}_{\mathbf{i}}\right)\right] \phi_{\alpha}\left(\mathbf{x}_{i}\right)=E_{\alpha} \phi_{\alpha}\left(\mathbf{x}_{i}\right)
$$

where $\phi_{\alpha}$ is the single particle wave function, and $E_{\alpha}$ is the energy in a given state characterized by the angular momentum, parity, and isospin. States with similar energies form a shell. Nucleons fill shells from the lowest energy up to the Fermi level $\left(k_{F} \approx 250 \mathrm{MeV}\right)$. An illustrative picture of the shell model is shown in Fig. 1-1.

The shell model provides complete information on the momentum distribution and number of protons or neutrons in each orbit (so-called occupation number). In order to compare those quantities to experimental data, the spectral function is introduced. 


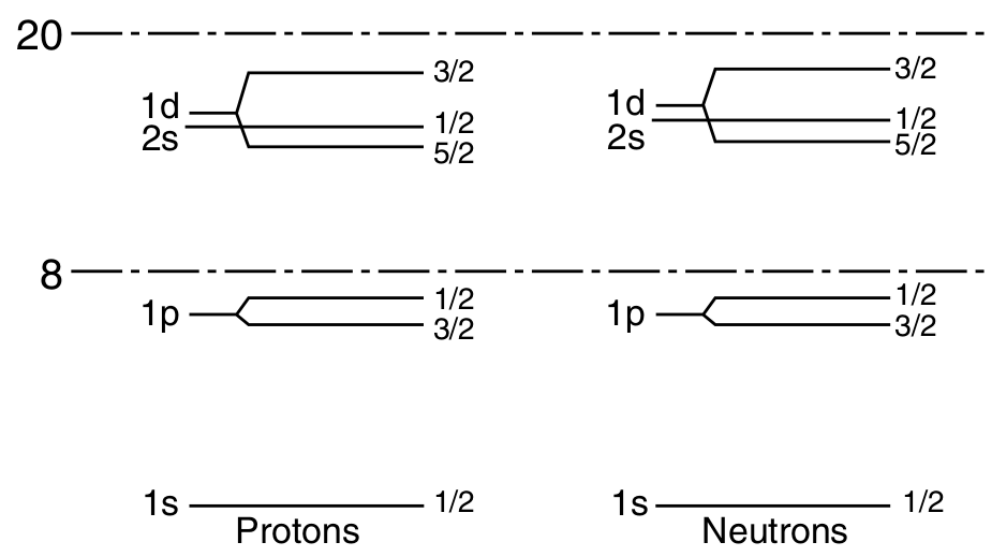

Figure 1-1: The lowest two energy shells in nuclei with odd mass number, Fig.17.6 from [6].

In Quasi-elastic $(\mathrm{QE})$ electron scattering, the incident electron with four-momentum $\left(E_{1}\right.$, $\mathbf{k}_{1}$ ) interacts with a single nucleon within the target nucleus, and knocks that nucleon out of the nuclear system. The four-momenta of the scattered electron $\left(E_{2}, \mathbf{k}_{2}\right)$ and the knockedoff nucleon $\left(E_{3}=\sqrt{\mathbf{p}_{X}^{2}+M_{N}^{2}}, \mathbf{p}_{X}\right)$ can be detected coincidentally in experiment. The subscript $X$ indicates that the state of that knockout particle is unknown. The scattering diagram with plane wave impulse approximation (PWIA) is shown in Fig. 1-2, Assuming no multi-step processes occur, the initial momentum $\mathbf{p}$ and the removal energy $E_{m}$ of the struck nucleon are:

$$
\begin{aligned}
\mathbf{p} & =\mathbf{p}_{X}-\mathbf{q}, \\
E_{m} & =M_{A-1}^{\star}+M_{N}-M_{A},
\end{aligned}
$$

where the three-vector momentum transfer $\mathbf{q}=\mathbf{k}_{1}-\mathbf{k}_{2} . M_{N}$ and $M_{A-1}^{\star}$ are the mass for the nucleon, and the excited residual nucleus respectively.

The spectral function 


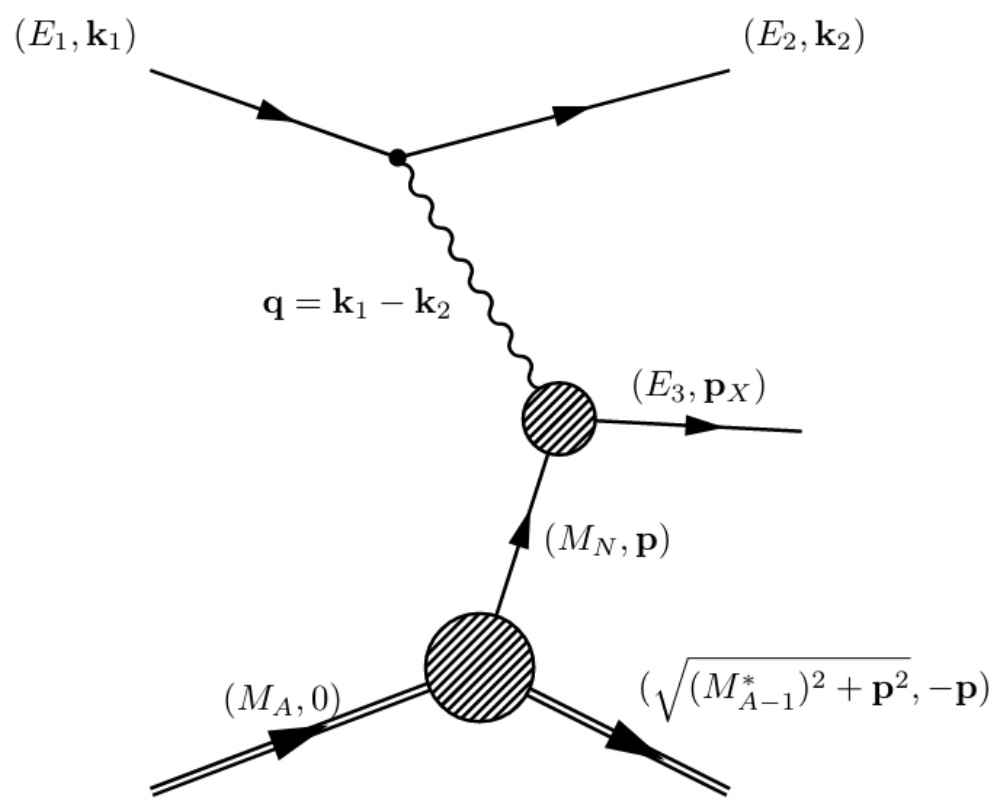

Figure 1-2: PWIA diagram of QE electron scattering off a nucleus $A$.

$$
S\left(\mathbf{k}, E_{m}\right)=\sum_{\alpha}\left|\phi_{\alpha}\right|^{2} \delta\left(E_{m}-E_{\alpha}\right)
$$

denotes the probability of finding a nucleon with momentum $\mathbf{k}$ and removal energy $E_{m}$ in the nucleus. Experimentally it can be deduced from the cross section data with the plane wave impulse approximation (PWIA) by using:

$$
\frac{d^{6} \sigma}{d \mathbf{p}_{X} d E^{\prime}}=K \sigma_{e p} S\left(\mathbf{k}, E_{m}\right)
$$

where $K$ is a kinematic factor obtained from the experiment, and $\sigma_{e p}$ is a model-dependent electron-proton cross section.

In IPSM, The nucleon momentum distribution at orbit $\alpha$ is $S\left(\mathbf{k}, E_{\alpha}\right)$. The occupation number for a closed orbit is $2 j+1$ where $j$ is the total angular momentum, that is, the 
chance of finding a nucleon with removal energy $E_{\alpha}$ from that orbit is

$$
\int S\left(\mathbf{k}, E_{m}=E_{\alpha}\right) d \mathbf{k}=2 j+1 .
$$

In other words, the spectroscopic strength $\frac{\int S\left(\mathbf{k}, E_{\alpha}\right) d \mathbf{k}}{2 j+1}=1$.

In the 1980s, a series of high precision single-nucleon knockout experiments were performed
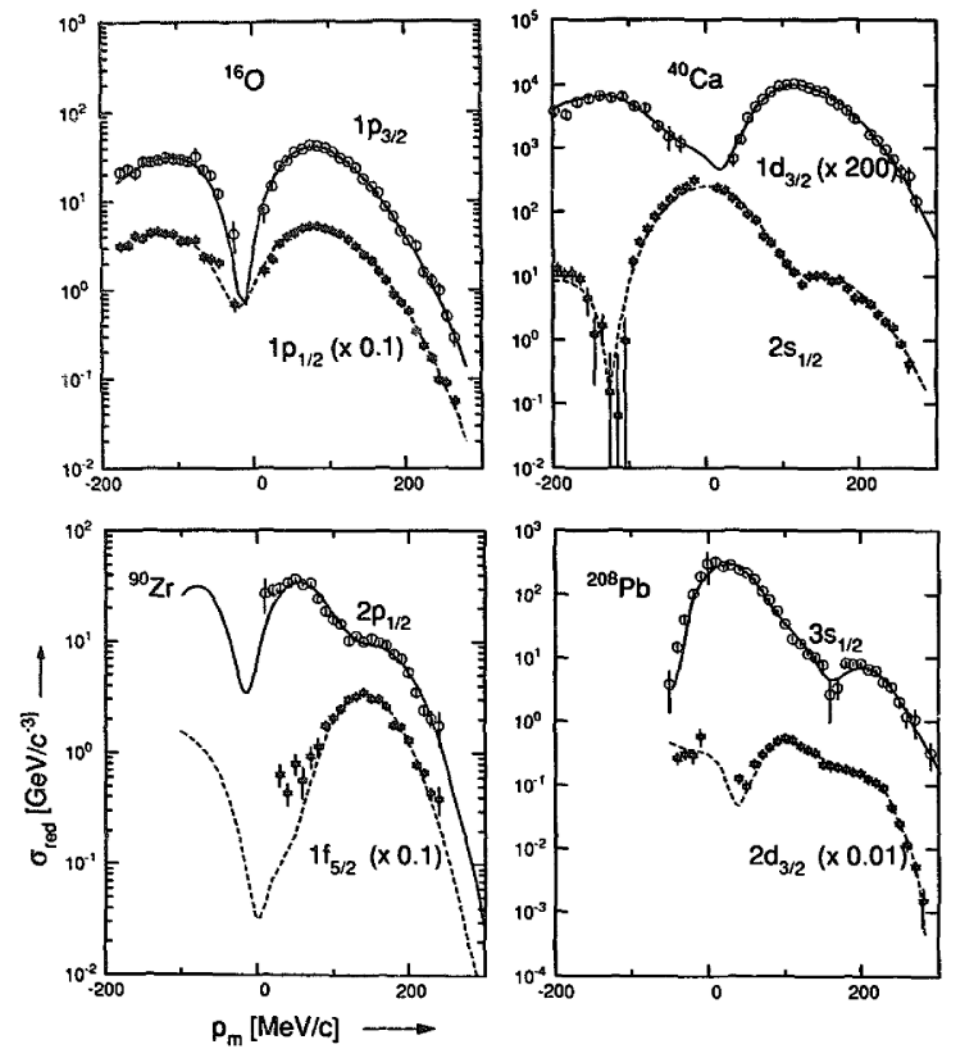

Figure 1-3: Momentum distributions from the (e,e $\left.\mathrm{e}^{\prime} \mathrm{p}\right)$ measurement of the valence shell (upper marks) and the next deeper shell (lower marks). Curves from Continuum Distorted Wave Impulse Approximation (CDWIA) calculations with Woods-Saxon bound-state wave functions are scaled to fit the data. Figure taken from [3].

at NIKHEF-K with a 500-MeV electron beam [3]. Fig. 1-3 shows the measured momentum distribution in valence shells (the outermost shell just below Fermi level) and the next-deeper 
shells from several closed-shell nuclei. The shapes of the nucleon momentum distribution in a given orbit predicted by the mean field theory match the experimental data well up to the Fermi momentum $k_{F} \approx 250 \mathrm{MeV} / \mathrm{c}$. However, results from the same experiment (see Fig. 1-4) also show that the spectroscopic strengths from various nuclei were between 0.6 to 0.7 in valence orbits. Non-zero strength for states just above the Fermi level were also observed [3].

Although this less-than-one spectroscopic strength contradicts the mean field theory prediction, it is actually in line with the nuclear matter calculations which use a realistic nucleon-nucleon interaction model that includes short-range and tensor correlations [3]. Later calculation of the nucleus momentum distribution

$$
n_{A}(\mathbf{k})=\sum_{\alpha} S\left(\mathbf{k}, E_{\alpha}\right)
$$

shows that the nucleon-nucleon $(\mathrm{NN})$ correlated pair with a very high relative momentum and a low total momentum is essential to produce the high-momentum and high removal energy part of the spectral function (See Fig. 1-5).

\subsubsection{NN Interactions}

The IPSM assumes no correlations between nucleon wave functions. But the realistic nucleon wave functions are overlapping. In the simplest configuration, two free nucleons interact with each other and generate a potential. Decades of approaches have been made to obtain the realistic NN potential (see Fig. 1-6). For example, the widely used Argonne v18 (AV18) parameterization [9] includes the electromagnetic force and pion exchange at long to intermediate range, and a charge-independent hard repulsive core from the direct fit of NN scattering experimental data. The one-pion exchange model, which successfully 


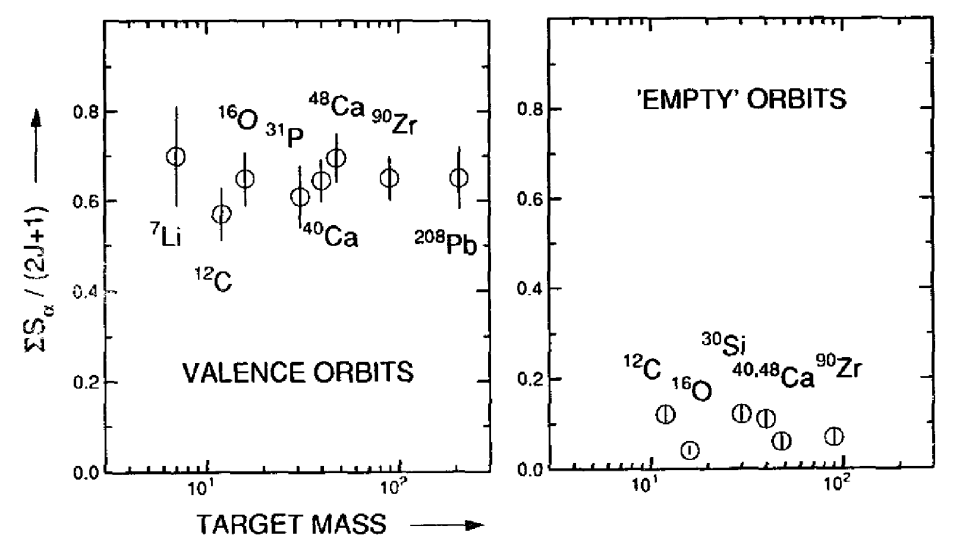

Figure 1-4: Spectroscopic strength for valence orbitals (left) and for states just above the Fermi level (right) as measured at NIKHEF-K [3].

describes the NN interaction at long distance $(r>2 \mathrm{fm})$, has a tensor contribution

$$
S_{12}=2\left[3 \frac{(\vec{S} \cdot \vec{r})^{2}}{r^{2}}-\vec{S}^{2}\right]
$$

where $\vec{S}$ is the total spin. The Pauli exclusion principle requires the wave function of a twonucleon system to be anti-symmetric, that is, the sum of total orbital angular momentum $L$, total spin $S$, and total isospin $T$ should be an odd number. As $L=0,2$, the possible two-nucleon configurations are:

- $S=1, T=0:$ Deuteron-like $n p$ pair,

- $S=0, T=1: \quad n n, n p, p p$ pairs.

As shown in Fig. 1-7, this tensor term yields an attractive force with the spin $S=0$, isospin $T=1$, orbital angular momentum $L=0$ configuration which is responsible for the deuteron bound state. As a result, the proton and neutron are more likely to pair up in short-range than $p p$ or $n n$. Calculation shows that in the tensor-dominant and D-wave dominant region $\left(1.5 \mathrm{fm}^{-1} \leq q \leq 3 \mathrm{fm}^{-1}\right)$, the density of $n p$ pairs is much larger than that of $p p$ pairs as 

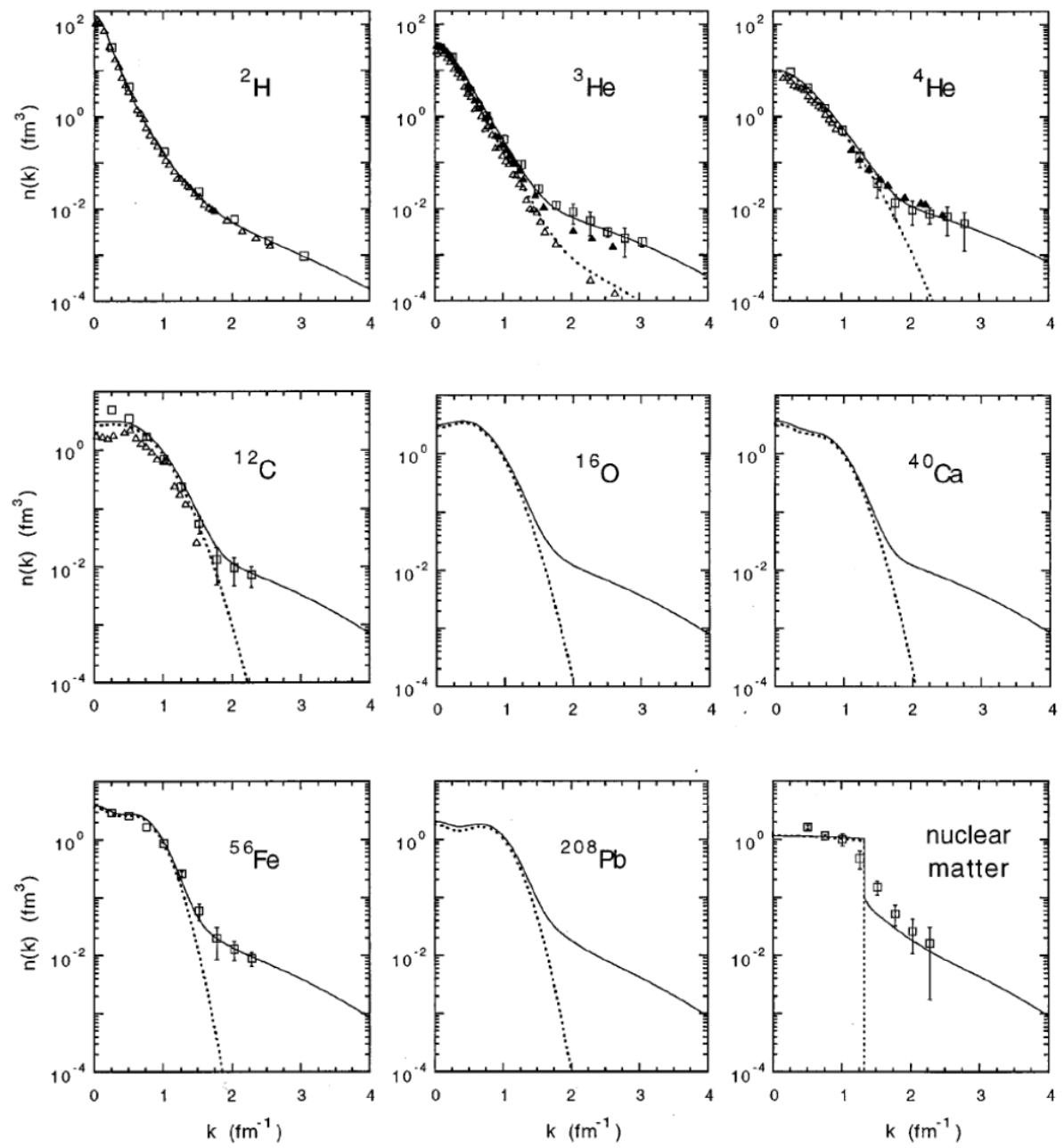

Figure 1-5: Nucleon momentum distributions from experimental data (symbols), shell model calculation (dotted line), and calculation including short-range correlation (solid line) [2]. $1 \mathrm{fm}^{-1} \approx 200 \mathrm{MeV} / \mathrm{c}$. 


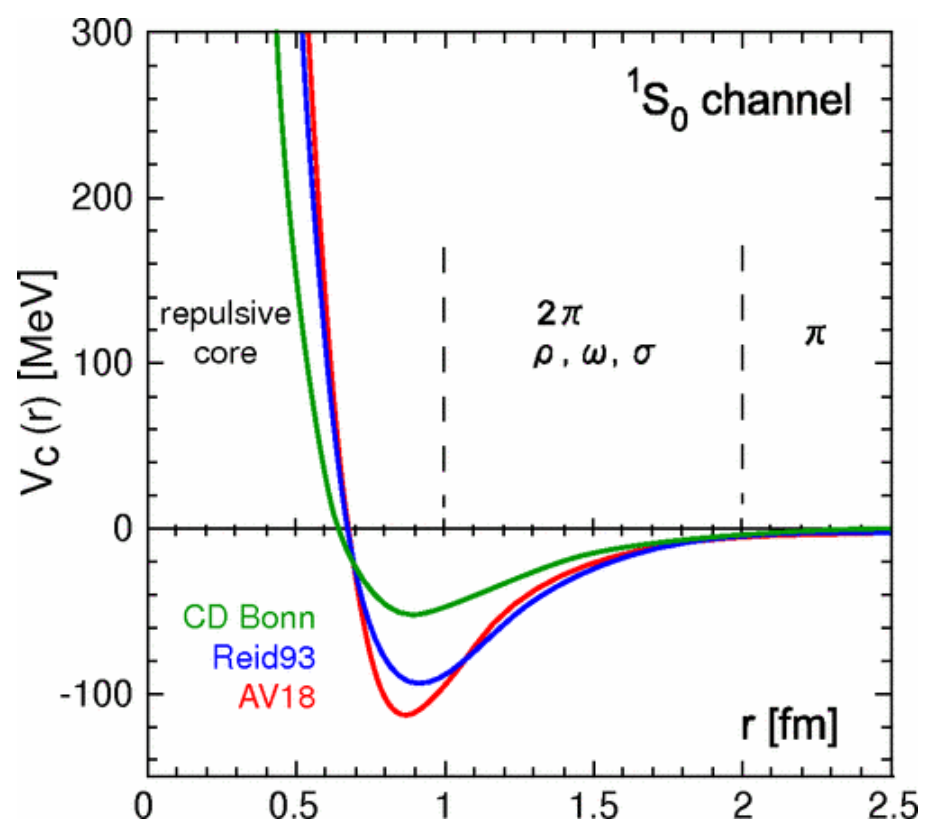

Figure 1-6: Three examples of modern NN potentials in the ${ }^{1} S_{0}$ (spin singlet and s-wave) channel: CD-Bonn [7], Reid93 [8], and AV18 [9]. Figure taken from [10].

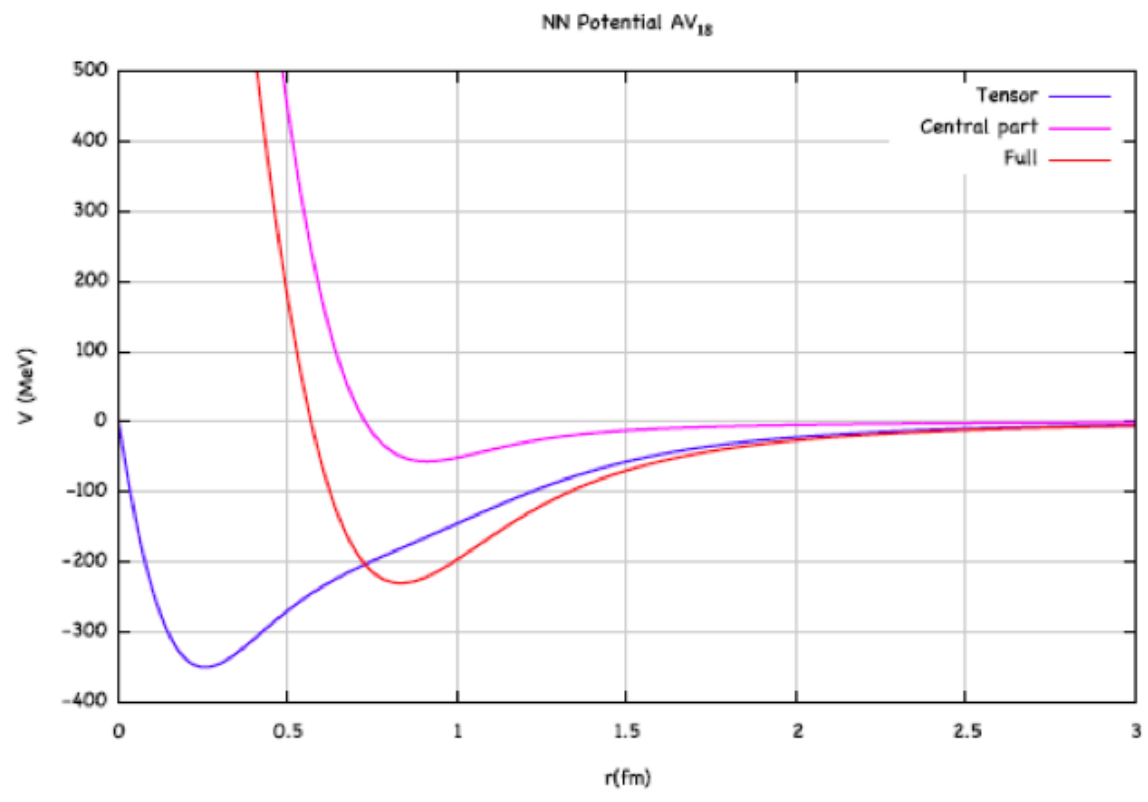

Figure 1-7: The NN potential calculated from AV18 [11]. 
shown in Fig. 1-8 [12].

Calculations with NN potentials alone cannot reproduce the binding energies of nuclei. They

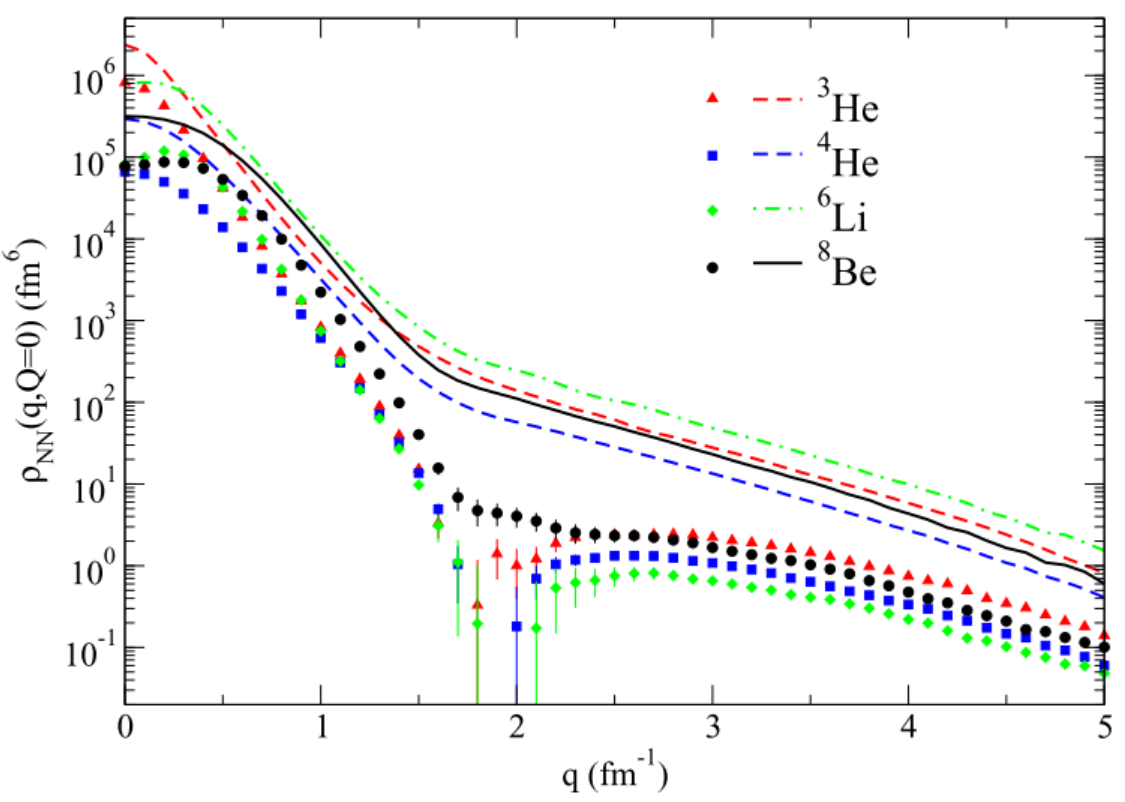

Figure 1-8: The momentum distributions of $n p$ (lines) and $p p$ (symbols) pairs with relative momentum $q$ and total momentum $Q=0$ [12].

are usually combined with some three-nucleon $(3 \mathrm{~N})$ potentials such as the Urbana X (UX) potential [13]. One restriction of the empirical NN potential fitting is that the short-range interaction terms are not sensitive to the NN scattering data at low momentum transfer. In recent years Chiral Effective Field Theory Calculations of up to next-to- next-to-nextto-leading order $(\mathrm{N} 3 \mathrm{LO})$ are developed to describe the $\mathrm{NN}$ interaction at short distance. However these calculations have not been able to match the experimental data (see Ref.[14]). 


\subsection{Short-range Correlations in Nuclei}

\subsubsection{Scaling variables for QE scattering}

\section{y scaling}

In the PWIA, the one-nucleon knockout cross section in a nucleus $A$ can be written as the integral of electron-nucleon cross sections over phase space:

$$
\begin{aligned}
\frac{d^{2} \sigma(q, \omega)}{d \omega d \Omega} & =\sum_{N=1}^{A} \int d E_{m} \int d^{3} p S\left(p, E_{m}\right) \sigma_{e N} \delta\left(\omega+M_{A}-E_{3}-M_{A-1}^{*}\right) \\
& =2 \pi \sum_{N} \int d E_{m} \int d p p S\left(p, E_{m}\right) \hat{\sigma}_{e N}\left|\frac{\partial \omega}{k \partial \cos \alpha}\right|^{-1}
\end{aligned}
$$

where $\omega=E_{1}-E_{2}$ is the electron energy transfer, $\Omega$ is the solid angle. $\cos \alpha=(\mathbf{q} \cdot \mathbf{p}) /(|\mathbf{q}||\mathbf{p}|)$. $\hat{\sigma}_{e N}\left(q, \omega, p, E_{m}\right)$ is the elastic cross section of an electron scattered off an off-shell nucleon, and

$$
\frac{\partial \omega}{p \partial \cos \alpha}=\frac{q}{\left(M^{2}+q^{2}+p^{2}+2 p q \cos \alpha\right)^{1 / 2}} .
$$

In the limit of high momentum transfer where the excitation energy and the transverse nucleon momentum $p_{\perp}$ is negligible, equation 1.11 can be factorized as:

$$
\frac{d^{2} \sigma(q, \omega)}{d \omega d \Omega}=\left(Z \hat{\sigma}_{e p}\left(q, \omega, p_{\text {min }}, E_{\text {min }}\right)+N \hat{\sigma}_{e n}\left(q, \omega, p_{\text {min }}, E_{\text {min }}\right)\right)\left|\frac{d \omega}{k \partial \cos \alpha}\right|^{-1} F(q, \omega)
$$

where the nuclear structure function

$$
F(q, \omega)=2 \pi \sum_{N} \int d E_{m} \int d p p S\left(p, E_{m}\right)
$$


Now introduce the scaling variable that satisfies:

$$
\omega+M_{A}=\sqrt{M^{2}+(q+y)^{2}}+\sqrt{M_{A-1}^{2}+y^{2}},
$$

we have

$$
\begin{aligned}
F(y) & =2 \pi \sum_{N} \int_{E_{\text {min }}}^{\infty} d E_{m} \int_{\left|y-\left(E_{m}-E_{m i n}\right)\right|}^{\infty} d p p S\left(p, E_{m}\right) \\
& =\frac{\sigma}{\left(Z \hat{\sigma}_{e p}+N \hat{\sigma}_{e n}\right)} \frac{q}{\left(M^{2}+q^{2}+p^{2}+2 p y\right)^{1 / 2}}
\end{aligned}
$$

Note that $y$ is very similar but should not be confused with $\mathbf{p}_{\|}$[15]. Fig. 1-9 shows the extracted $F(y)$ from JLAB and SLAC data, with the peak centered at $y=0$ corresponds to QE scattering. At the high momentum transfer side of the peak $\left(y<0, x_{b j}>1\right)$, datasets from different $Q^{2}$ show the same scaling behavior.

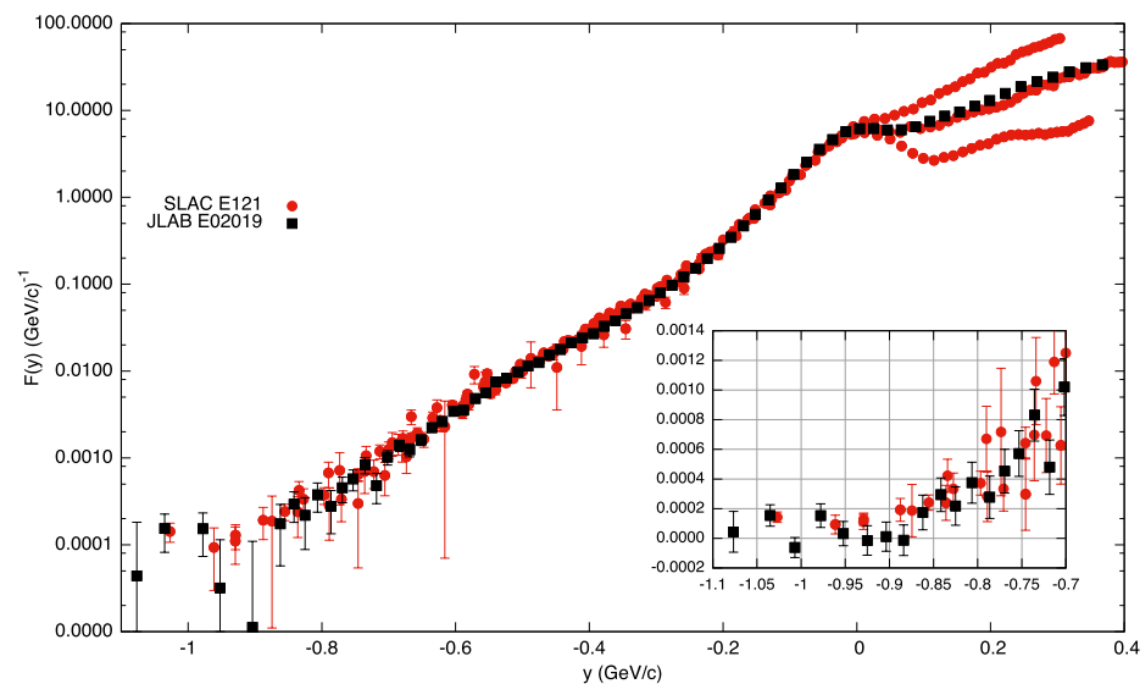

Figure 1-9: $F(y)$ extracted from ${ }^{3} \mathrm{He}$ cross sections. Data are taken from SLAC and JLAB. The inset shows $F(y)$ at $0.7>y>1.1$ which corresponds to the region of interest for $3 \mathrm{~N}-$ SRCs. For purely QE scattering $F(y)$ is symmetric about $y=0$. Data on the right side of the peak show deviations due to inelastic contributions. Figure taken from [16]. 


\section{Bjorken x Scaling}

The inclusive QE electron scattering cross section can be written as a function of the scattered electron momentum $E^{\prime}$ and scattering angle $\theta$

$$
\frac{d^{2} \sigma}{d E^{\prime} d \theta}=\sigma_{M o t t}\left[W_{2}^{A}\left(E^{\prime}, \theta\right)+2 \tan ^{2} \frac{\theta}{2} W_{1}^{A}\left(E^{\prime}, \theta\right)\right]
$$

with the Mott cross section $\sigma_{M o t t}=\alpha^{2} \cos ^{2}(\theta / 2) / 4 E^{2} \sin ^{4}(\theta / 2)$ and nuclear response functions $W_{1}, W_{2}$. Here $Q^{2}=-\left(\mathbf{p}_{X}-\mathbf{p}\right)^{2}$ is the squared four-momentum transfer. A schematic drawing of the shape of total electron cross section as a function of $\omega$ is shown in Fig. 1-10. For convenience we often rewrite Eq. 1.17 in terms of $Q^{2}$ and $x_{b j}$, where the Bjorken variable

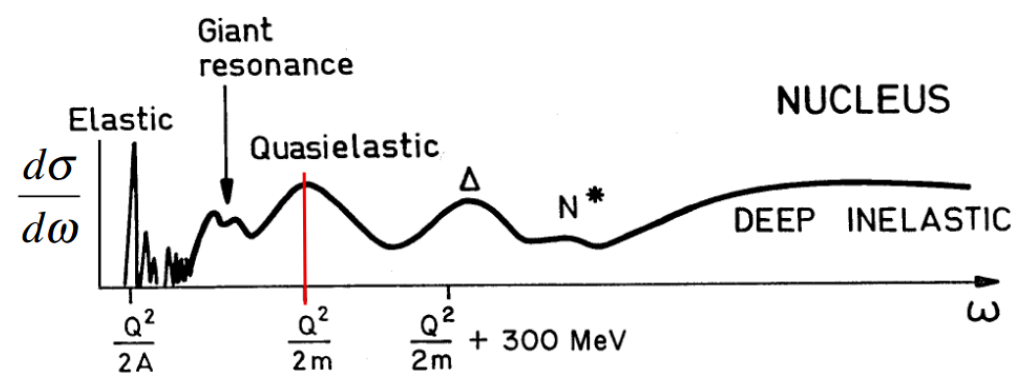

Figure 1-10: Schematic drawing of (e,e $\left.\mathrm{e}^{\prime}\right)$ total cross section as a function of energy transfer. With fixed beam energy $x_{b j}$ decreases with $\omega . \omega=Q^{2} / 2 m$ is the QE peak. Figure taken from [17].

$x_{b j}=\frac{Q^{2}}{2 M_{N} \omega}$ is a popular scaling factor in the deep inelastic scattering study. In the infinite momentum frame, $x_{b j}$ represents the fraction of momentum carried by the parton that interacts with the electron in a nucleon. The invariant mass of that nucleon after scattering is $W^{2} c^{2}=(p+q)^{2}=M_{N}^{2} c^{2}+2 M_{N} \omega-Q^{2}$. In the limit of QE scattering, $W^{2}=M_{N}^{2}$ so that $x_{b j}=\frac{Q^{2}}{2 M_{N} \omega}=1$. 


\subsubsection{Isospin dependence in NN SRCs}

\section{Triple-coincidence experiments}

When two nucleons interact at short distance $(r<1 \mathrm{fm})$, the repulsive core of the NN potential pushes them away from each other. In this instance those two nucleons have high relative momentum (back-to-back) above the Fermi level, but as a pair it has small central momentum and remains in ground state. As shown in Fig. 1-11, when a high momentum electron is scattered off a nucleon in the SRC pair, it not only knocks out the struck nucleon, but also free the paired nucleon. Signatures of such an event include high initial momentum of the scattered nucleon $\left(|\mathbf{p}|>k_{F}\right)$ and a coincident detection of two nucleons with the scattered electron.

The scattered electron, the knocked-off nucleon and its correlated partner could be

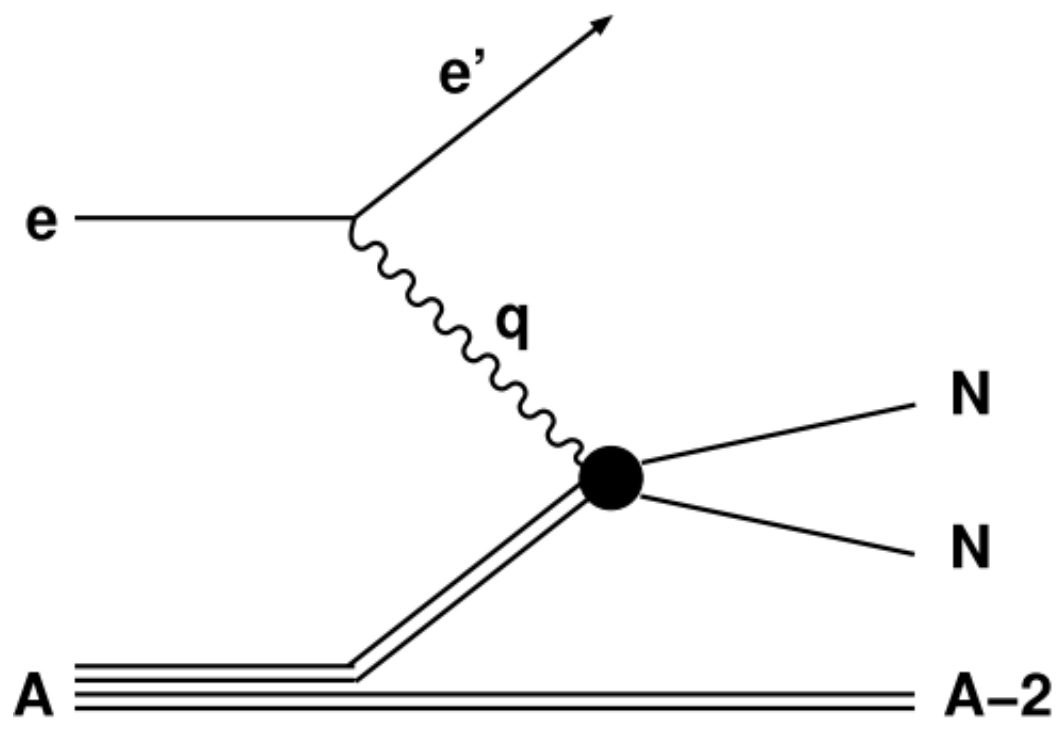

Figure 1-11: Feynman diagram of electron scattering on the SRC pair. Figure 17 from [18]. 


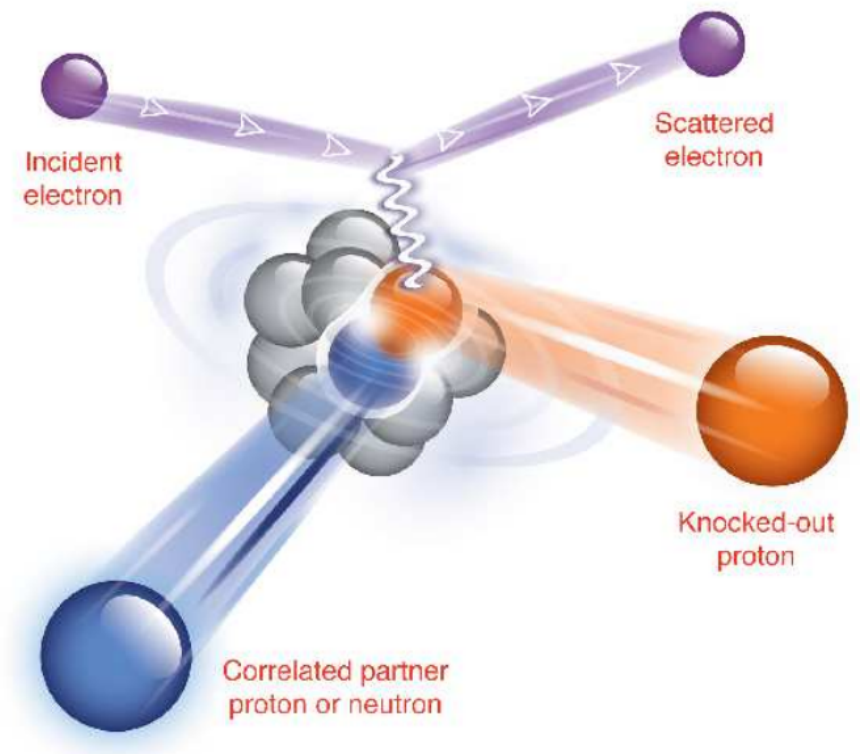

Figure 1-12: Illustratiion of $\mathrm{C} 12\left(\mathrm{e}, \mathrm{e}^{\prime} \mathrm{pN}\right)$ reaction. [4]

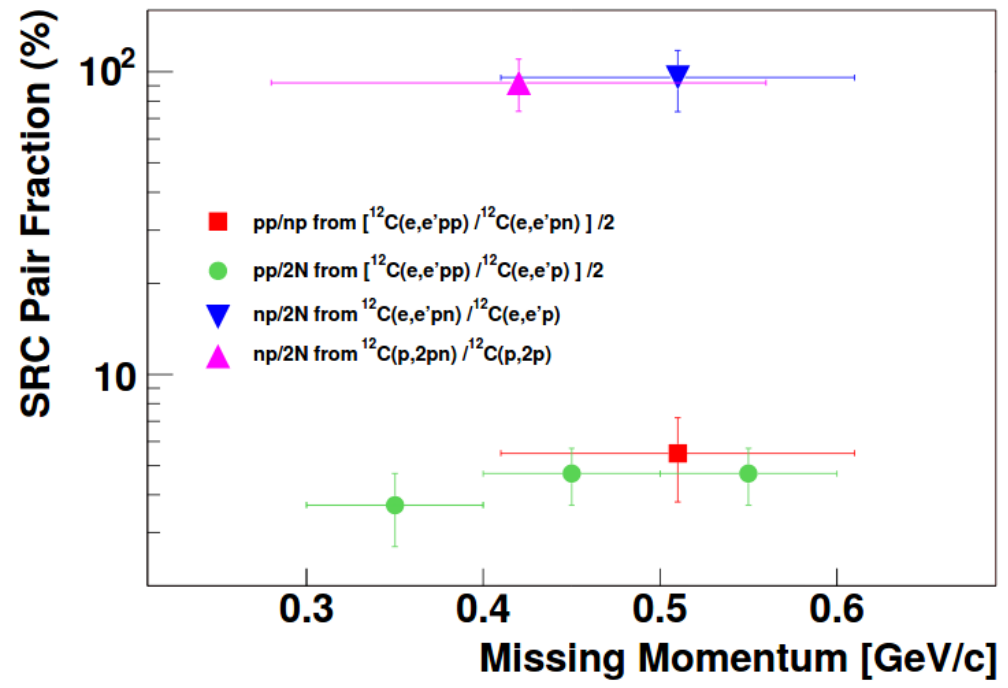

Figure 1-13: $n n, n p$, and $p p$ pair fractions in ${ }^{12} \mathrm{C}$ from JLAB E01-015 experiment [4]. 


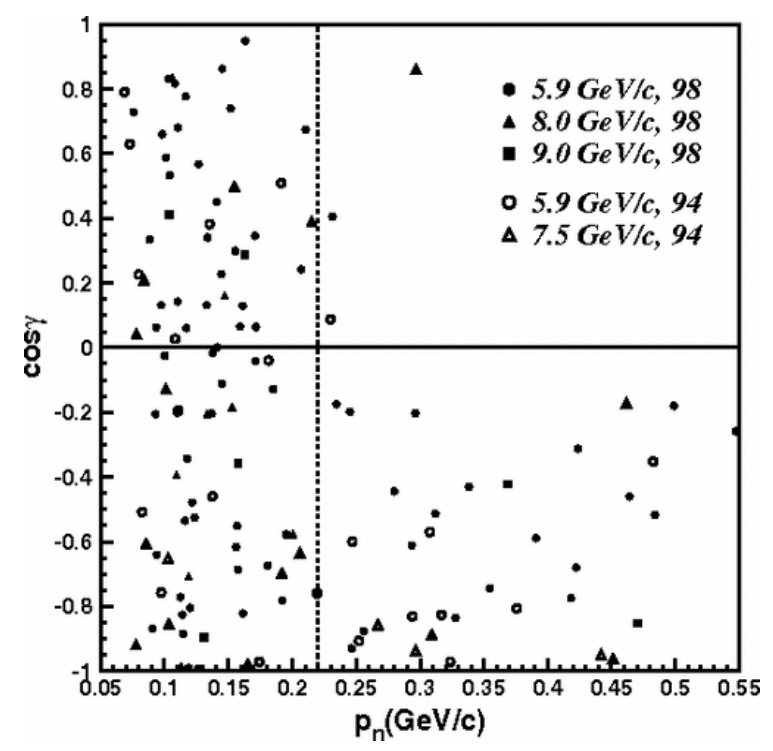

Figure 1-14: the angle between the initial momentum of the scattered proton and the observed momentum of its paired neutron $\mathbf{p}_{n}$ as a function of the neutron momentum. Markers indicate data with different beam energies.

detected simultaneously in a triple-coincidence experiment(see Fig. 1-12). Measurements from the E01-015 experiment at JLAB observed that among events with reconstructed high momentum protons, there was almost always a correlated nucleon. $90 \%$ of the time the correlated pairs were $n p$ pairs, and the rest were proton-proton $(p p)$ or neutron-neutron $(n n)$ pairs (see Fig. 1-13). The experiment is a follow-up to the ${ }^{12} \mathrm{C}\left(\mathrm{p}, \mathrm{p}^{\prime} \mathrm{pn}\right) \mathrm{X}$ experiment at Brookhaven National Lab [19] which demonstrated a strong back-to-back directional correlation between the initial momentum of the scattered proton and the observed momentum of its paired neutron above Fermi level (see Figure 1-14).

Though suffering from low statistics, the observed dominance of high momentum $n p$ pair with back-to-back momentum in those triple-coincidence experiments provided strong evidences of the dominance of the tensor forces in NN SRCs. It cannot be explained by competing processes such as nucleon Final State Interactions (FSI) and Meson Exchange 
Currents (MEC) which cannot have strong isospin preference.

\section{Inclusive Experiments}

Given that the high momentum nucleons are mostly from the $n p$ SRC pairs, they should in principle behave in the same way in all nuclei. This explains the universal shape of high momentum tails from various targets as seen in Fig. 1-5. It also suggests that for an inclusive measurement at the NN SRC dominant kinematics, the per-nucleon cross section ratio of a target nucleus with $A$ nucleons relative to that of ${ }^{2} \mathrm{H}$ should be the same up to a constant: $\sigma_{A} / \sigma_{2} H \approx a_{2}(A)$, where the ratio $a_{2}(A)$ is the relative probability of finding $n p$ pairs in the nucleus $A$ to the deuteron. For example, the carbon to deuteron plateau height is $\sim 5$ in Fig. 1-16. Given that there are $\sim 4 \%$ of high momentum nucleons in deuteron [18, the probability of finding high momentum nucleons in carbon is $4 \% \times 5=20 \%$. Note that for heavy nuclei the motion of pairs can enhance this ratio [20]. Fig. 1-15 shows the minimum initial momentum of the struck nucleon with respect to $x_{b j}$ at fixed $Q^{2}$. According to this calculation, the high momentum nucleons could be detected at $x_{b j}<0.5$ or $x_{b j}>1.4$. The former is dominated by the inelastic scattering contribution. On the other hand, the one-nucleon knockout cross section at $x_{b j}>1$ falls off rapidly, so in the region of $1.4<x_{b j}<2$ the cross section from electron scattering on SRC pairs are significant. The final state interaction (FSI), meson exchange current (MEC) and intermediate resonances could obscure the SRC measurement. Generally, maintaining high $Q^{2}$ and low energy transfer would suppress those competing processes. Note that FSI is still significant even with high $Q^{2}$, but the effect is believed to be limited within the SRC pair [2]. The impact of FSI will be mostly cancelled when taking the ratio of inclusive QE cross sections.

The cross section ratios $\sigma_{A} / \sigma_{2} H$ from JLAB E02-019 Experiment clearly show plateaus (see 
Fig. 1-16 at $1.4<x_{b j}<1.8$. This scaling behavior provides a direct link to the isospin configurations of SRC from inclusive cross section ratios. $a_{2}(A)$ can be calculated from the height of the plateau with corrections for inelastic processes and momentum smearing.
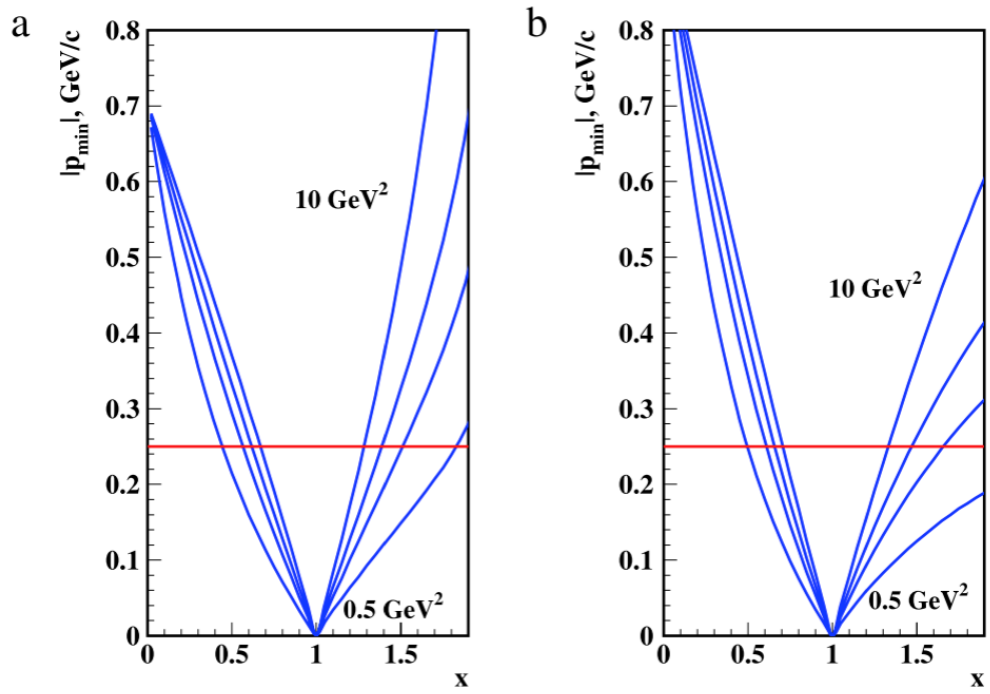

Figure 1-15: The relation between $p_{m}$ in and $x_{b j}$ in electron scattering from ${ }^{2} \mathrm{H}$ (left) and $\mathrm{Au}$ (right) at $Q^{2}=0.5,1.5,3$, and $10 \mathrm{GeV} / \mathrm{c}^{2} \cdot p_{\min }$ is the minimum parallel momentum of the initial nucleon. Red lines indicate the Fermi momentum. Figure taken from [18].

\subsection{The E12-11-112 Experiment}

\subsubsection{Physics Goals}

The E12-11-112 experiment at Hall A, Jefferson Lab is designed to precisely determine the isospin dependence at $\mathrm{NN}$ and $3 \mathrm{~N}$ SRCs region in $\mathrm{A}\left(\mathrm{e}, \mathrm{e}^{\prime}\right) \mathrm{X}$ scattering with deuterium $\left({ }^{2} \mathrm{H}\right)$, tritium $\left({ }^{3} \mathrm{H}\right)$ and helium-3 $\left({ }^{3} \mathrm{He}\right)$ targets. The kinematics covers $0.7<x_{b j}<3$ and $0.4<Q^{2}<3 \mathrm{GeV}^{2}$. The $\mathrm{A}=3$ mirror nuclei tritium (1 proton and 2 neutrons) and helium3 (2 protons and 1 neutron) is the simplest many body system for ab-initio calculations 


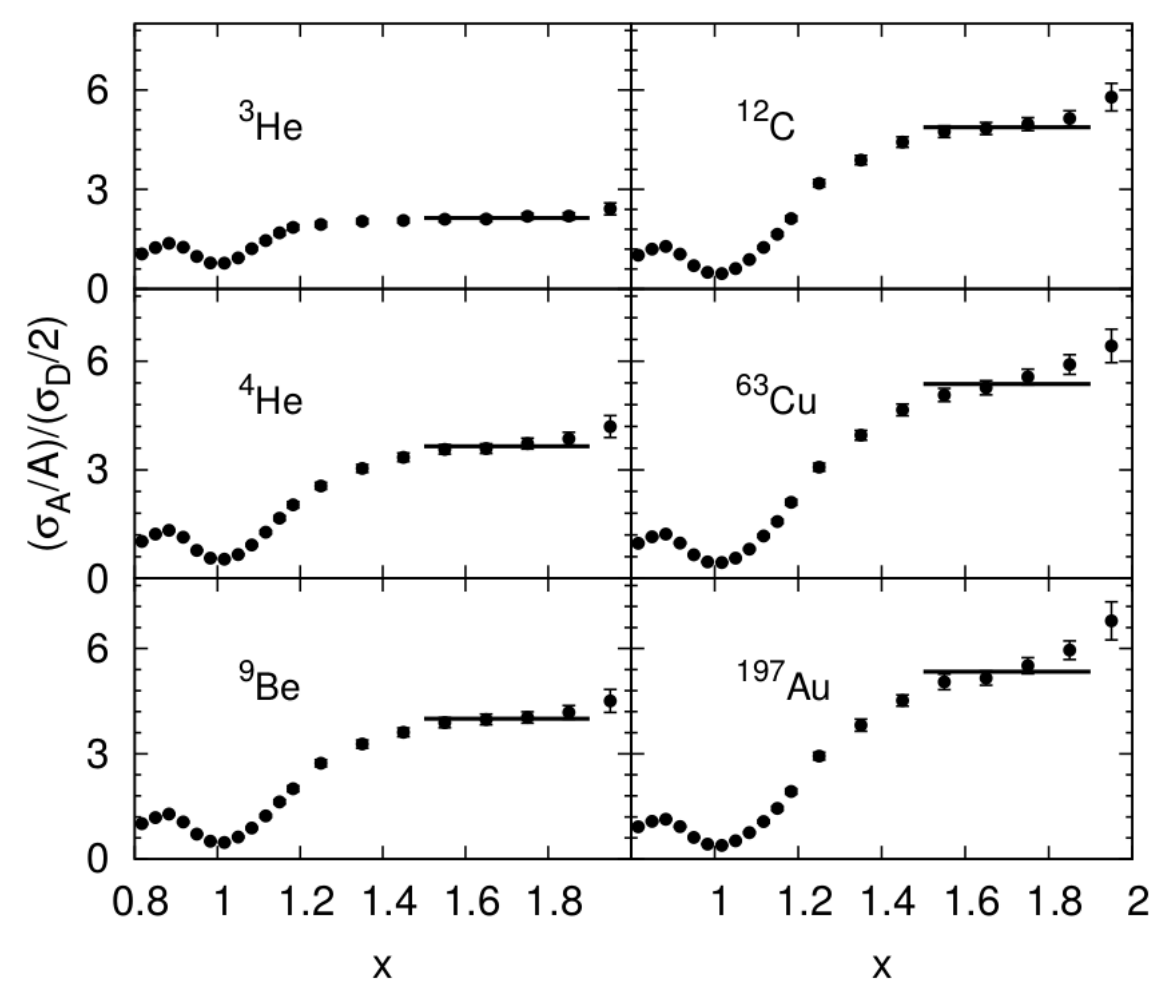

Figure 1-16: The per-nucleon cross section ratios of various nuclei to deuterium from JLAB E02-019 experiment [20]. In general the ratios are flat between $1.4<x_{b j}<1.8$ in light nuclei. In heavy nuclei the smearing of the central momentum of the SRC pair is more complicated such that a direct observation of plateau is difficult, But $a_{2}(A)$ can still be extracted from the cross section ratio to provide information on the isospin dependence. 
including FSI and MEC effects. It allows all NN configurations with maximized isospin asymmetry, and also provides a chance to study the two most possible and naturallyoccurred 3N SRC configurations.

\section{Probe Isospin Dependence in NN SRCs}

The primary physics goal of this experiment is to study the isospin dependence in NN SRCs. The major part of production data were taken with $4.3 \mathrm{GeV}$ electron beam at a scattering angle of 17 degrees, which correspond to an average $Q^{2}=1.4 \mathrm{GeV}^{2} / c^{2}$ at $1.4<x_{b j}<2$. This $Q^{2}$ is large enough to probe nucleons above Fermi momentum and to suppress competing processes, but not too large to kill the $\mathrm{QE}$ cross section which drops faster than $1 / Q^{4}$. Similar to previous inclusive SRC experiments, the ${ }^{3} \mathrm{H} /{ }^{2} \mathrm{H}$, and ${ }^{3} \mathrm{He} /{ }^{2} \mathrm{H}$ per-nucleon ratios are expected to be flat in the NN SRC dominant region $\left(1.4<x_{b j}<2\right)$ so that $a_{2}(A=3)$ can be calculated from the height of the plateau. Moreover, taking the ratios of tritium and helium-3 cross sections from the same experiment and kinematics would provide a direct comparison of the NN SRC configurations in tritium and helium-3. Tritium and helium-3 have similar binding energy hence nuclear smearing effect. Other competing processes such as FSI and MEC would be very similar for those two nuclei if not already suppressed by the kinematics. Therefore the ${ }^{3} \mathrm{H}$ to ${ }^{3} \mathrm{He}$ raw cross section ratio at $1.4<x_{b j}<2$ can be seen as the direct ratio of the NN SRC contributions from two nuclei. Most systematical uncertainties would be cancelled in the cross section ratio so that the result could achieve high precision.

If simply counting the possible neutron and proton combinations in the nucleus, ${ }^{3} \mathrm{He}$ (two $n p$ pair and one $p p$ pair) and ${ }^{3} \mathrm{H}$ (two $n p$ pair and one $n n$ pair) have the same probability to form $n p$ pairs. If the $2 \mathrm{~N}$ SRCs are strongly isospin-dependent, i.e., $n p$ pairs are dominant as 
shown in triple-coincidence experiments, their cross section ratios in the $2 \mathrm{~N}$ plateau region $\left(1.4<x_{b j}<2\right)$ will be

$$
\frac{\sigma_{3} H}{\sigma_{3} H e}=\frac{\sigma_{n p}+\sigma_{n}}{\sigma_{n p}+\sigma_{p}} \approx \frac{\sigma_{n p}}{\sigma_{n p}}=1 .
$$

If there is indeed no isospin-preference, the cross section ratio should be

$$
\frac{\sigma_{3} H}{\sigma_{3} H e}=\frac{2 \sigma_{n}+\sigma_{p}}{\sigma_{n}+2 \sigma_{p}} \approx 0.7 \text { to } 0.75
$$

with $\sigma_{p} / \sigma_{n} \approx 2.5$ to 3 in our kinematic range. The measured ${ }^{3} \mathrm{H} /{ }^{2} \mathrm{He}$ cross section ratios are expected to sit in between this two limits with $2 \%$ level total uncertainty. This would provide precise information on fractions of $n p$ and $p p$ SRC pairs in the $\mathrm{A}=3$ system.

\section{Check the $Q^{2}$ dependence in NN SRCs}

From Fig. $1-15$ we can see that the onset of NN SRC plateau between $1<x_{b j}<2$ depends on $Q^{2}$. If $Q^{2}$ is too small, the inclusive electron scattering has lost its sensitivity to high momentum nucleons. This matches the experiment observation as shown in Fig. 1-17. With the wide $Q^{2}$ range in this experiment, we can make a similar measurement of this transition. Because the deviation from scaling at low $Q^{2}$ comes from effects such as FSI and MEC, we would expect their impact to be smaller in the ratio of two very similar nuclei (tritium and helium-3) than in the heavier nuclei to deuteron ratio. Data in the transition region could be used to test the limit of theory models. The final $Q^{2}$ cut for the NN SRC ratio study should be chosen such that the shape and height of the plateau are independent of $Q^{2}$. 

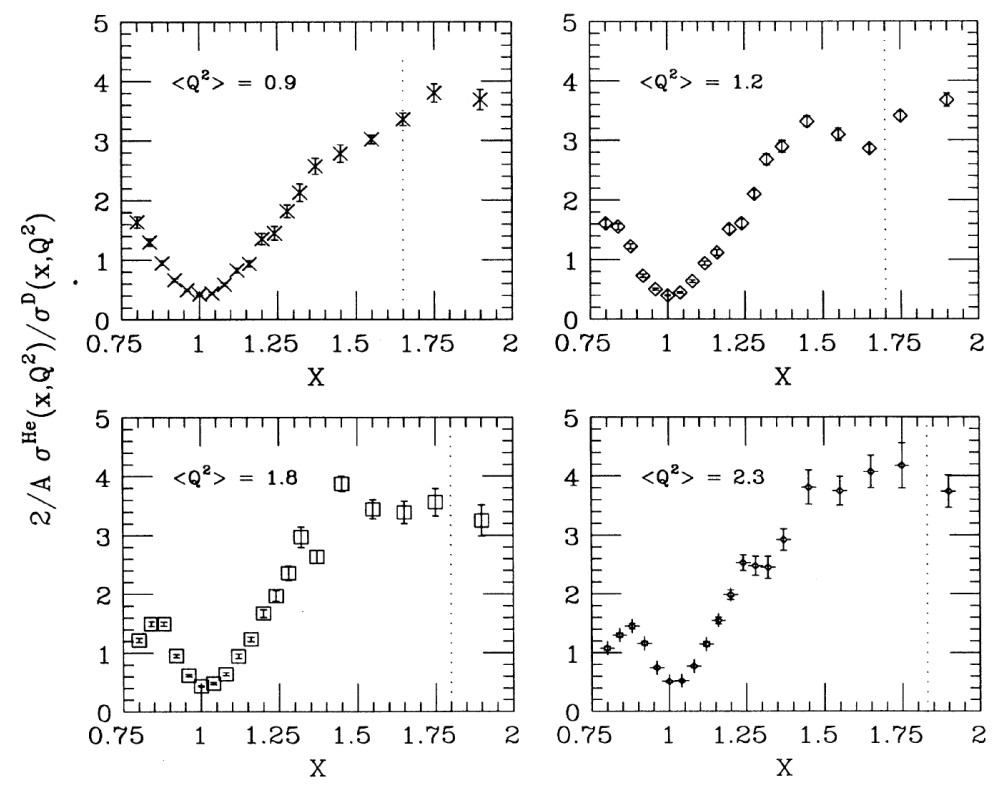

Figure 1-17: The ${ }^{4} \mathrm{He}$ to ${ }^{2} \mathrm{H}$ cross section ratio with respect to $x_{b j}$ at $Q^{2}=0.9-2.3$ $\mathrm{GeV}^{2} / c^{2}$ 21]. The vertical dashed line on each panel correspond to a final state that is $50 \mathrm{MeV}$ greater than the deuteron rest mass. Data were taken at SLAC [22].

\section{Explore the possible 3N SRCs}

Tritium and helium- 3 are stable $A=3$ systems in nature. They are the best place to find the possible 3N SRC configurations such as ${ }^{3} \mathrm{H}$-like or ${ }^{3} \mathrm{He}$-like clusters. Similar to NN SRCs, the $3 \mathrm{~N}$ SRC contributions to inclusive cross sections are expected to be significant in the $2<x_{b j}<3$ region. Good evidence for 3N SRCs would be a $\sigma_{A} / \sigma_{3}$ plateau. Without a tritium target, previous experiments took ratios of heavy nuclei to ${ }^{3} \mathrm{He}$ to search for $3 \mathrm{~N}$ SRC plateau. Results from different experiments show large discrepancies (see Fig. 1-18). Our experiment would allow a similar cross section ratio analysis with precise ${ }^{3} \mathrm{He},{ }^{3} \mathrm{H}$ cross section data and their iso-scalared average $\left(\left(\sigma_{3} \mathrm{He}_{e}+\sigma_{3} \mathrm{H}\right) / 2\right)$. Also, the absolute cross sections at $x_{b j}>2$ could be used to calculate the nucleon momentum distribution at $k>600$ $\mathrm{MeV} / \mathrm{c}$. 
Should a plateau be observed, the ratio $\sigma_{3} H / \sigma_{3} H e$ would cast light on the $3 \mathrm{~N}$ SRCs isospin structure. Two possible 3N configurations are shown in Fig. 1-19. In case (a) the struck nucleon 3 has greater momentum. If the $3 \mathrm{~N}$ structure is isospin-insensitive, the ratio depends on the nucleon and proton numbers, which yields $\frac{2 \sigma_{n}+\sigma_{p}}{\sigma_{n}+2 \sigma_{p}} \approx 0.7$. If the nucleon 3 tends to be the single nucleon, the ratio $\sigma_{3}{ } / \sigma_{3} H_{e} \approx \sigma_{p} / \sigma_{n} \approx 3$. If nucleon 3 is one of the doubly-occurring nucleons instead, then the ratio becomes $\sigma_{n} / \sigma_{p} \approx 0.35$. In case (b) the configuration is symmetric and not sensitive to isospin, so the ratio will again $\approx 0.7[23$. To summarize, the cross section ratio will be close to 0.7 if $3 \mathrm{~N}$ SRCs are isospin-independent.

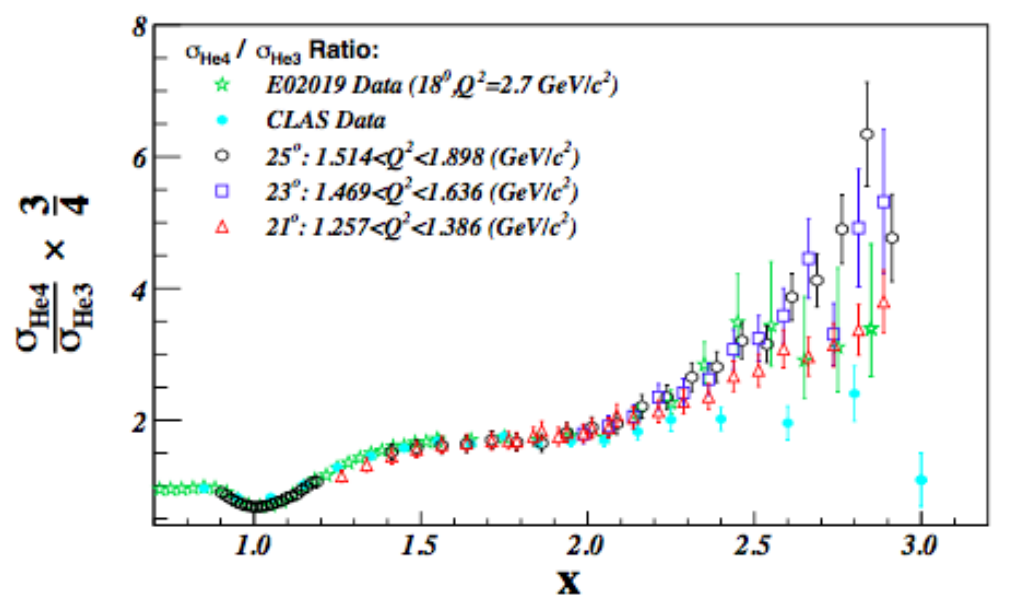

Figure 1-18: Cross section ratios of heavy nuclei over helium-3 from previous inclusive measurements. Results are inconsistent at $x_{b j}>2$. [11] 


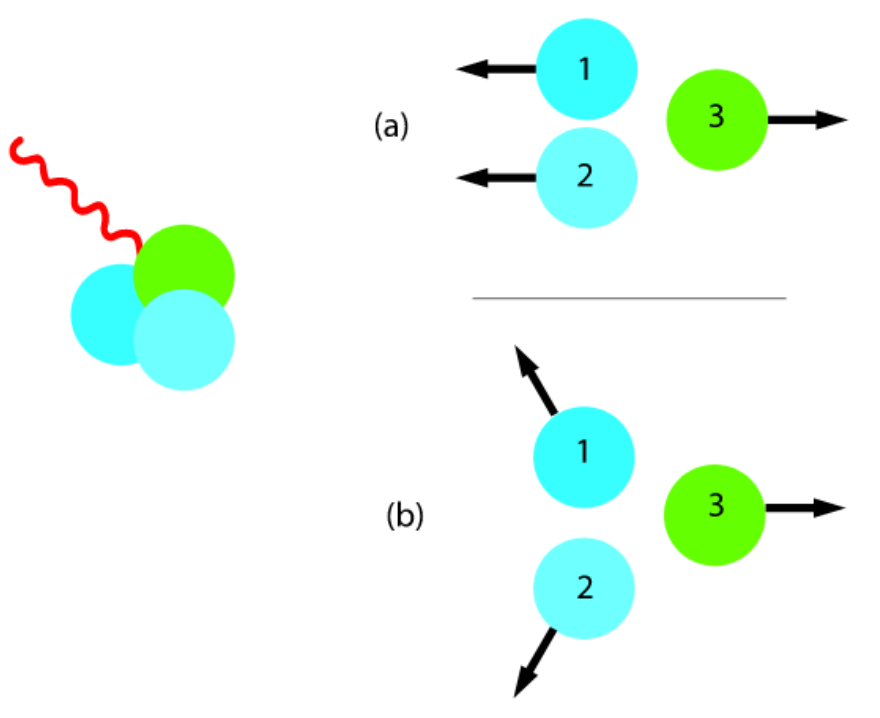

Figure 1-19: Illustration of two extreme case of possible 3N SRCs configurations. 23]

\subsubsection{Connections to Other Experiments}

\section{E08-14 experiment}

The JLAB experiment E08-14 measured the inclusive cross sections of ${ }^{2} \mathrm{H},{ }^{3} \mathrm{He},{ }^{4} \mathrm{He},{ }^{12} \mathrm{C}$, ${ }^{40} \mathrm{Ca}$ and ${ }^{48} \mathrm{Ca}$ at $Q^{2}=0.8-2.8 \mathrm{GeV} / \mathrm{c}^{2}$ at $1<x_{b j}<3$. Scaling behaviors at NN SRC region were observed. No $3 \mathrm{~N}$ SRCs plateau was seen in the $\mathrm{A} /{ }^{3} \mathrm{He}$ ratios, which is inconsistent with earlier measurements from CLAS (see Fig. 1-18). Recent reanalysis of CLAS data suggests that the observed plateaus at $x_{b} j>2$ could be an analysis issue caused by the limited momentum resolution of the CLAS detector [24]. The E08-14 experiment also extracted NN SRC ratios of calcium isotopes ${ }^{48} \mathrm{Ca} /{ }^{40} \mathrm{Ca}$ (see Fig. 1-20). This result can help test the hypothesis of $n p$ dominance by comparing the calcium isotopes of similar mass but with different isospin structure [25]. 


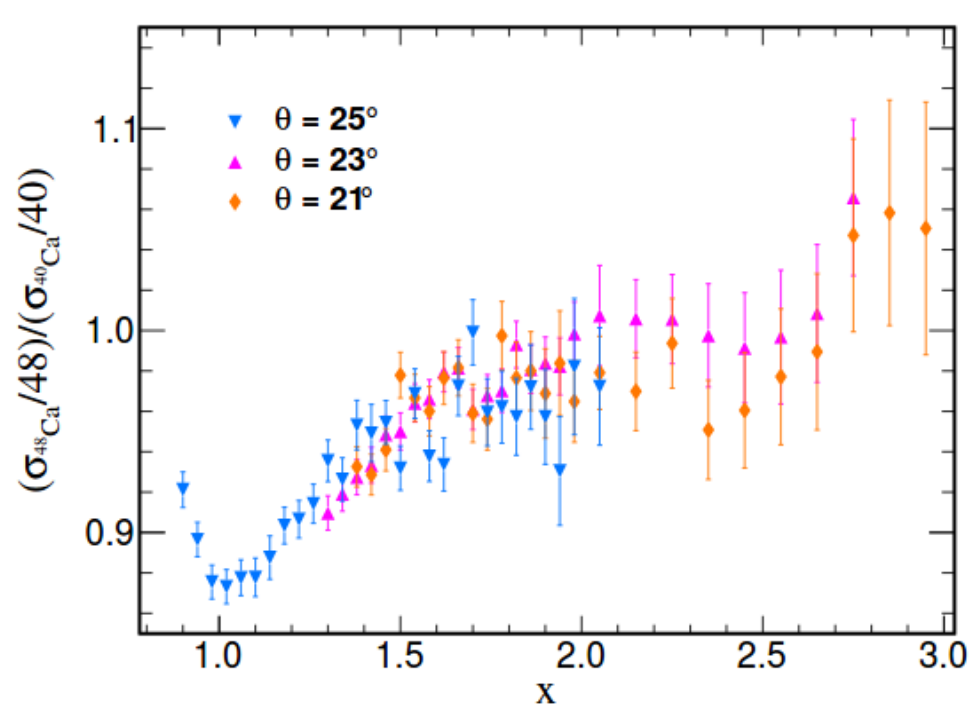

Figure 1-20: Per-nucleon cross section ratios of ${ }^{48} \mathrm{Ca} /{ }^{40} \mathrm{Ca}$ from experiment E08-14 [25].

\section{Other Tritium Experiments at JLAB}

This experiment was carried together with another four tritium-related measurements (E1214-009, E12-14-011, E12-10-103, E12-17-003). All experiments ran in 2018 in Hall A, Jefferson Lab. Though the observables and configurations are very different, those tritium experiments shared the same gas tritium target system and detector calibrations.

The E12-10-103 experiment (MARATHON) is trying to extract the structure function $F_{2}^{n} / F_{2}^{p}$ ratio, eventually the $d / u$ valence quark ratio from the tritium to helium-3 cross section ratios in deep inelastic scattering [27]. The tritium (helium-3) to deuterium ratios can also be used to study the A-dependence of EMC effect. The so-called EMC effect describes the deflation of per-nucleon cross section in heavy nuclei as compared to deuterium at $0.3<x_{b j}<0.7$ as shown in Fig. $1-21$. Both the slope of the EMC ratio and the height of NN SRC plateau ratio increase with the nuclear mass. Some analysis suggested that those two ratios are linearly correlated (see Fig. 1-22). The tritium (helium-3) to deuterium EMC 

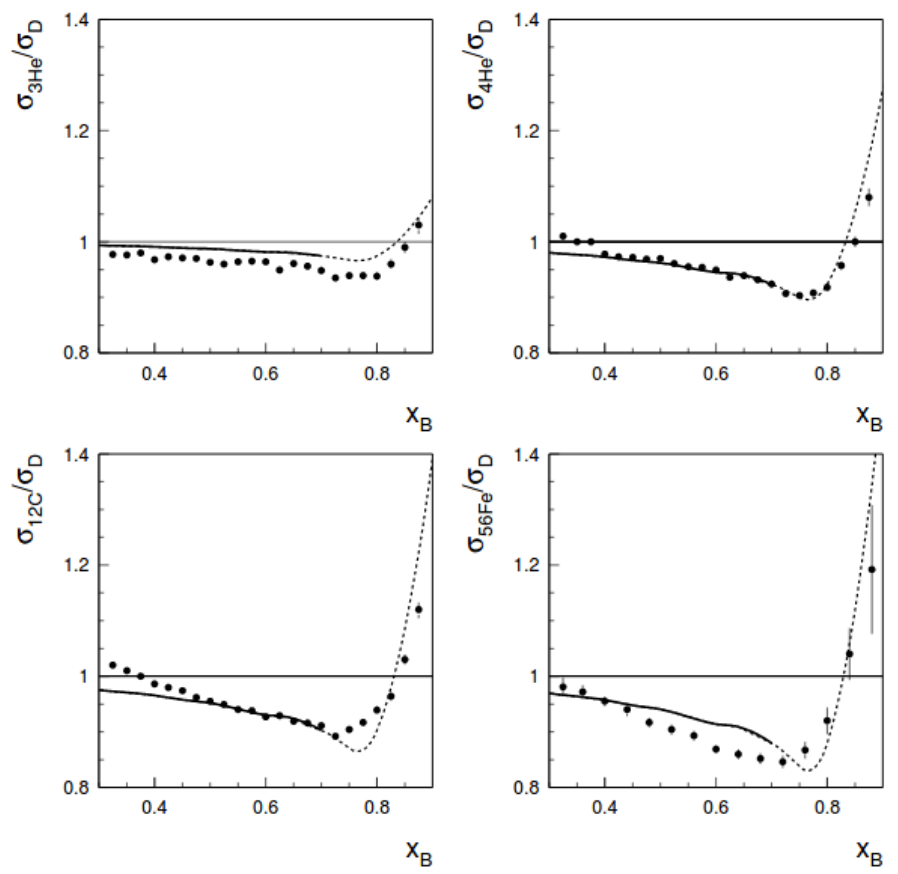

Figure 1-21: Nuclei to deuterium per-nucleon cross section ratios. Figure taken from [26]

ratio from the MARATHON experiment and the $a_{2}(A=3)$ discussed in this thesis could be combined to provide more information on the relation between EMC and SRC.

The E12-14-011 experiment studies the tritium and helium-3 momentum distribution with $\left(\mathrm{e}, \mathrm{e}^{\prime} \mathrm{p}\right)$ scattering. It focuses on the high momentum tails $\left(k>k_{F e r m i}\right)$ generated by SRC [28. They also collected the inclusive cross section data at $1<x_{b j}<2$ which will be discussed in the following sections. 

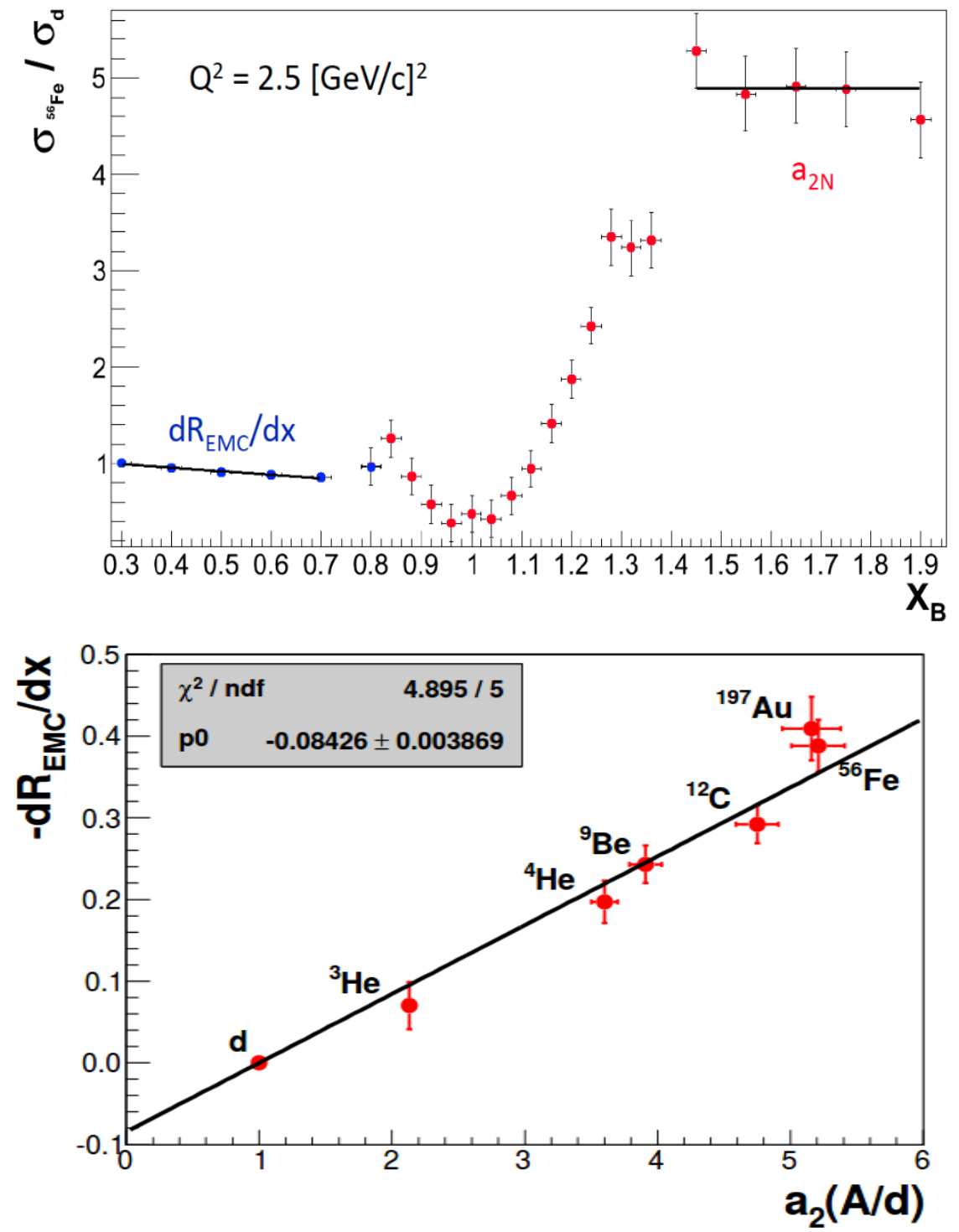

Figure 1-22: Top: Illustration of SRC and EMC ratios from [26]. Botom: A linear fit of EMC ratio versus $a_{2}(A)$ from [29]. 


\section{Chapter 2}

\section{Experiment Setup and Calibrations}

\section{$2.1 \quad$ E12-11-112 Overview}

The Tritium program at Hall A, Jefferson Lab ran from December of 2017 to the end of 2018. On December 152018 the first beam was sent to the tritium target for a target density study, followed by the MARATHON experiment (E12-10-103) and the e'p experiment (E12-14-011). This experiment (E12-11-112) started taking production data on May 22019. During the first period, we collected 5 days of low $Q^{2}$ data with $2.2 \mathrm{GeV}$ beam. The second run period started on September 26 2019. We took 33 days of high $Q^{2}$ dat with $4.3 \mathrm{GeV}$ beam.

As shown in Table 2.1, a list of beam energy, spectrometer angle, and spectrometer momentum settings (hereafter referred as "kinematic settings" or simply "kinematics") were used to cover a wide range of $x_{b j}$ and $Q^{2}$ range (see Fig. 2-1). Note that the $Q^{2}$ value in Table 2.1 is quoted at the QE peak. The actual $Q^{2}$ at the NN SRC dominant region would be slightly higher as shown in the plot. Two Hall A High Resolution Spectrometers (HRS) were used to take inclusive electron scattering data independently. Targets including hydrogen, deuterium, tritium, helium-3 gas in almost identical cells, and also thin carbon and titanium foils. The Spring 2018 kinematics were optimized for the $G_{M}^{n}$ measurements at $0.5<Q^{2}<1 \mathrm{GeV}^{2}$. At each HRS angle, multiple momentum settings were used to cover 
the full shape of QE peaks. Some low $Q^{2}$ kinematics also have acceptance at the QE tails $\left(x_{b j}>1.4\right)$ and were used to study the $Q^{2}$ dependence in NN SRCs. During the Fall 2018 run period, the Left HRS (LHRS) was parked at 17 degrees for dedicated SRC measurements that covered from $1<x_{b j}<3$. These are our main physics kinematics to extract the tritium to helium-3 cross section ratios at 2N SRC and 3N SRC region. In parellel with the LHRS data-taking, the Right HRS (RHRS) was rotated from 24 degrees to 28 degrees to continue the $G_{M}^{n}$ measurements at larger $Q^{2}$. We also took some opportunistic helium-3 and tritium elastic data at $Q^{2}=0.4 \mathrm{GeV}^{2}$ for the nuclear charge form factor extraction, and some DIS data with $W^{2} \sim 3 \mathrm{GeV}^{2}$ that could be combined with MARATHON data for the $R=\sigma_{L} / \sigma_{T}$ extraction.

This thesis focuses on the ${ }^{2} \mathrm{H},{ }^{3} \mathrm{H}$, and ${ }^{3} \mathrm{He}$ data from LHRS for the $2 \mathrm{~N}$ SRC ratio analysis. There are additional inclusive $2 \mathrm{~N}$ SRC data with similar experiment configurations from the e'p experiment as they collected data from LHRS single-arm trigger as well. Those two kinematics (EP-PK and EP-SRC) are also included in the analysis. More information about the $\mathrm{e}^{\prime} \mathrm{p}$ experiment can be found in [30].

\section{$2.2 \quad$ Experiment site}

The Thomas Jefferson National Accelerator Facility (Jefferson Lab) located in Newport News, Virginia is a national laboratory created in 1984. Its scientific goal is to investigate the nuclei and hadron structure with the unique Continuous Electron Beam Accelerator Facility (CEBAF). As shown in Fig. 2-2, a continuous wave electron beam is injected into the superconducting RF linear accelerators (LINAC) and accelerated to $1.1 \mathrm{GeV}$, bent by

\footnotetext{
${ }^{2}$ Kinematics EP-PK and EP-SRC from the coincidence experiment E12-14-011 were taken with the Left HRS single-arm trigger.
} 


\begin{tabular}{|c|c|c|c|c|c|}
\hline Kinematics & Beam Energy (GeV) & Angle & Momentum & $Q^{2}$ & $\overline{x_{b j}}$ \\
\hline \multicolumn{6}{|l|}{ Fall 2017} \\
\hline L15-E1 & 2.222 & 15.004 & 2.051 & 0.6 & 3 \\
\hline L15-E2 & 2.222 & 15.004 & 2.212 & 0.6 & 3 \\
\hline \multicolumn{6}{|l|}{ Spring 2018} \\
\hline L21-LS & 2.222 & 21.778 & 1.839 & 0.6 & 1 \\
\hline L21-PK & 2.222 & 21.778 & 1.896 & 0.6 & 1 \\
\hline L21-HS & 2.222 & 21.778 & 1.972 & 0.6 & 1 \\
\hline L21-SRC & 2.222 & 21.778 & 2.012 & 0.6 & $1-2$ \\
\hline$\overline{\mathrm{L} 24-\mathrm{LS}}$ & 2.222 & 23.891 & 1.788 & 0.7 & $1-2$ \\
\hline L24-PK & 2.222 & 23.891 & 1.843 & 0.7 & $1-2$ \\
\hline L24-HS & 2.222 & 23.891 & 1.917 & 0.7 & $1-2$ \\
\hline$\overline{\mathrm{L} 25-\mathrm{LS}}$ & 2.222 & 25.952 & 1.737 & 0.8 & 1 \\
\hline L25-HS & 2.222 & 25.952 & 1.862 & 0.8 & 1 \\
\hline L25-PK & 2.222 & 25.952 & 1.790 & 0.8 & 1 \\
\hline$\overline{\mathrm{L} 28-\mathrm{LS}}$ & 2.222 & 28.006 & 1.685 & 0.9 & 1 \\
\hline L28-PK & 2.222 & 28.006 & 1.737 & 0.9 & 1 \\
\hline L28-HS & 2.222 & 28.006 & 1.788 & 0.9 & 1 \\
\hline$\overline{\mathrm{L} 30-\mathrm{LS}}$ & 2.222 & 30.001 & 1.633 & 1 & 1 \\
\hline L30-PK & 2.222 & 30.001 & 1.683 & 1 & 1 \\
\hline L30-HS & 2.222 & 30.001 & 1.737 & 1 & 1 \\
\hline L30-SRC1 & 2.222 & 30.001 & 1.830 & 1 & $1-2$ \\
\hline L30-SRC2 & 2.222 & 30.008 & 1.830 & 1 & $1-2$ \\
\hline$\overline{\mathrm{R} 42-\mathrm{LS}}$ & 2.222 & 42.025 & 1.340 & 1.6 & 1 \\
\hline R42-PK & 2.222 & 42.025 & 1.379 & 1.6 & 1 \\
\hline R42-HS & 2.222 & 42.025 & 1.420 & 1.6 & 1 \\
\hline EP-PK ${ }^{1}$ & 4.325 & 17.802 & 3.543 & 1.5 & 1 \\
\hline EP-SRव ${ }^{2}$ & 4.325 & 20.881 & 3.543 & 1.9 & $1-2$ \\
\hline \multicolumn{6}{|l|}{ Fall 2018} \\
\hline L17-PK & 4.330 & 17.009 & 3.570 & 1.4 & 1 \\
\hline L17-HS & 4.330 & 17.009 & 3.670 & 1.4 & 1 \\
\hline L17-SRC1 & 4.330 & 17.009 & 3.820 & 1.4 & $1-2$ \\
\hline L17-SRC2 & 4.330 & 17.009 & 3.930 & 1.4 & $2-3$ \\
\hline$\overline{\mathrm{L} 17-\mathrm{E}}$ & 1.168 & 17.009 & 1.128 & 0.4 & 3 \\
\hline R24-LS & 4.330 & 24.016 & 3.000 & 2.3 & 1 \\
\hline R24-PK & 4.330 & 24.016 & 3.100 & 2.3 & 1 \\
\hline$\overline{\mathrm{R} 26-\mathrm{LS}}$ & 4.330 & 26.003 & 2.900 & 2.6 & 1 \\
\hline R26-PK & 4.330 & 26.003 & 3.000 & 2.6 & 1 \\
\hline R26-HS & 4.330 & 26.003 & 3.100 & 2.6 & 1 \\
\hline$\overline{\mathrm{R} 28-\mathrm{DIS} 1}$ & 4.330 & 28.004 & 1.580 & 1.6 & 0.3 \\
\hline R28-DIS2 & 4.330 & 28.004 & 1.710 & 1.7 & 0.35 \\
\hline R28-DIS3 & 4.330 & 28.004 & 1.910 & 1.9 & 0.43 \\
\hline R28-PK & 4.330 & 28.004 & 2.700 & 3 & 1 \\
\hline R28-HS & 4.330 & 28.004 & 2.975 & 3 & 1 \\
\hline R28-SRC & 4.330 & 28.004 & 3.100 & 3 & $1-2$ \\
\hline
\end{tabular}

Table 2.1: List of kinematics. The first letter indicates L(eft) or R(ight) HRS. LS, PK, HS stand for lower momentum side, peak, and higher momentum side of the QE peak. Momentum in $G e V / c$, quoted $Q^{2}$ in $G e V^{2} / c^{2}$. Those settings can be categorized into DIS region $(x<1)$, quasi-elastic region $(x=1)$, SRC region $(1<x<3)$, and $\mathrm{A}=3$ nuclei elastic region $(x=3)$. The beam energy and spectrometer angle calibration will be discussed in following sections. 


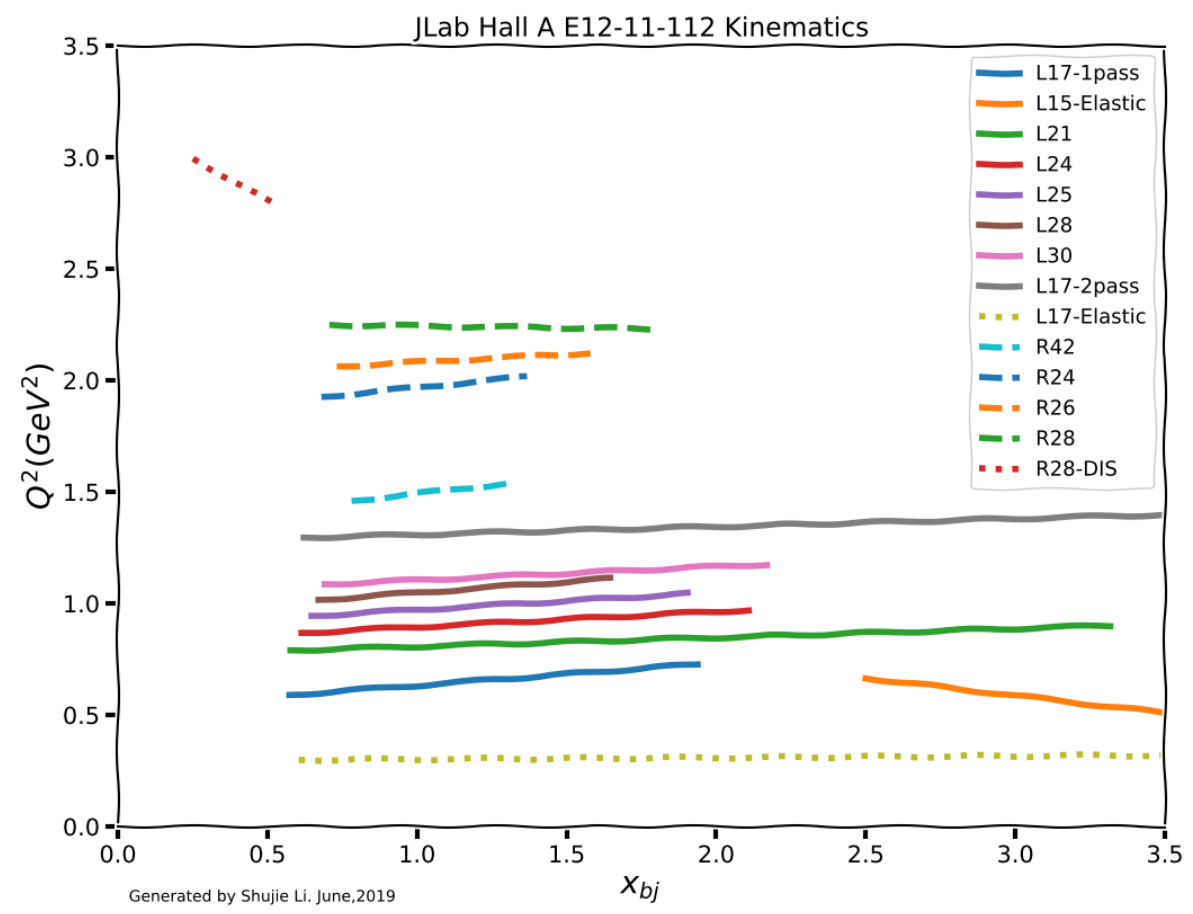

Figure 2-1: Kinematics coverage of the E12-11-112 experiment.

a semi-circular arc with bending magnets to enter another LINAC, then accelerated and bent. Each circle (pass) the beam energy increases by $2.2 \mathrm{GeV}$. After the $12 \mathrm{GeV}$ upgrade on 2015, CEBAF can now deliver up to 5 pass $(10.5 \mathrm{GeV})$ beam to experimental Hall A, $\mathrm{B}$, and $\mathrm{C}$, and (with additional half pass acceleration) $11.7 \mathrm{GeV}$ to Hall D simultaneously at a luminosity of $200 \mu \mathrm{A}$ in total.

Among the four halls, Hall A is the largest in dimension. A schematic of Hall A side view is shown in Fig. 2-3. It is the home for two high resolution spectrometers (HRS) with up to $10^{-4}$ momentum resolution but small solid angle acceptance. This matches the precision requirement for this experiment. 


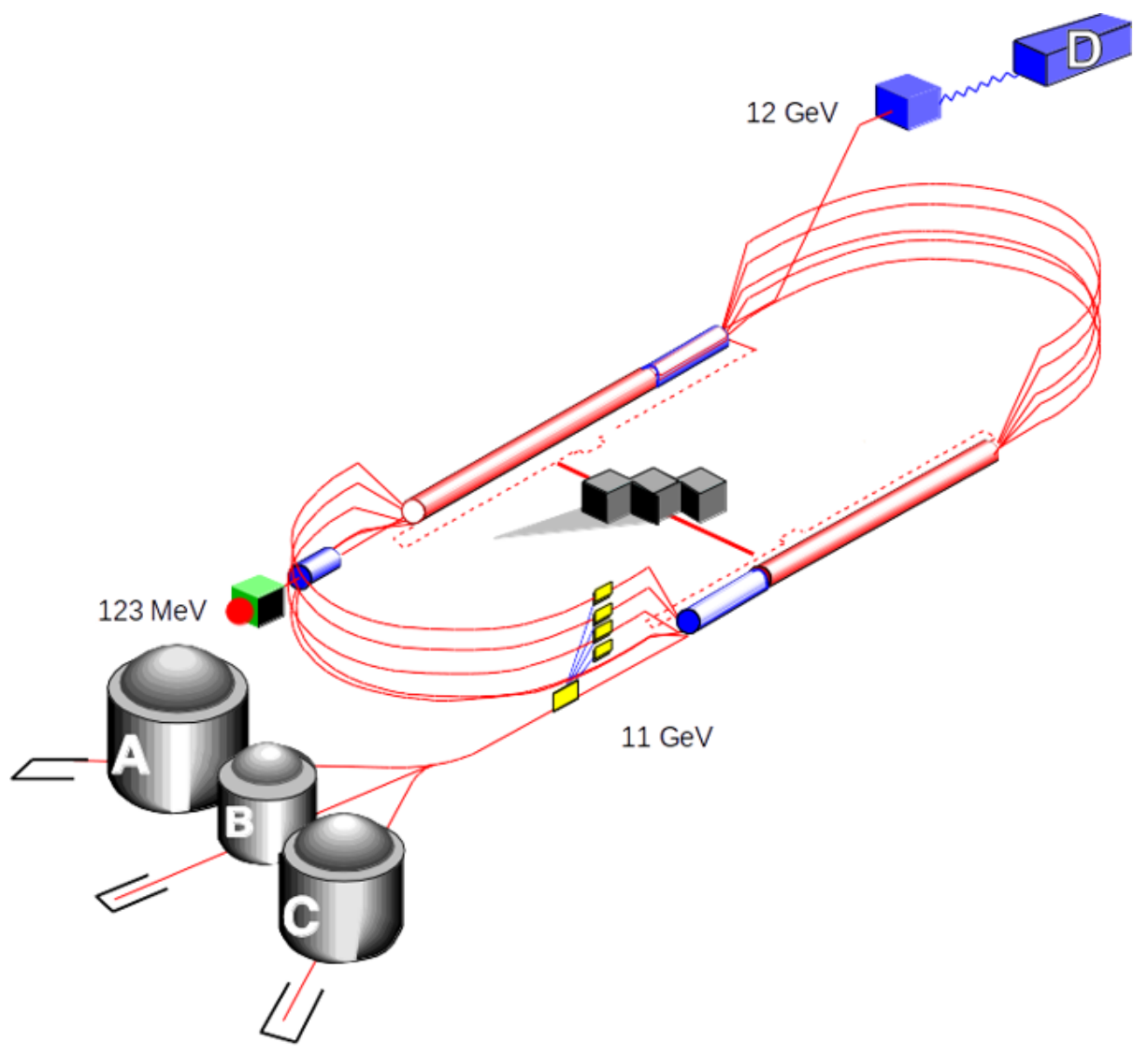

Figure 2-2: Schematic of CEBAF after the $12 \mathrm{GeV}$ upgrade. Figure 1 from [31.

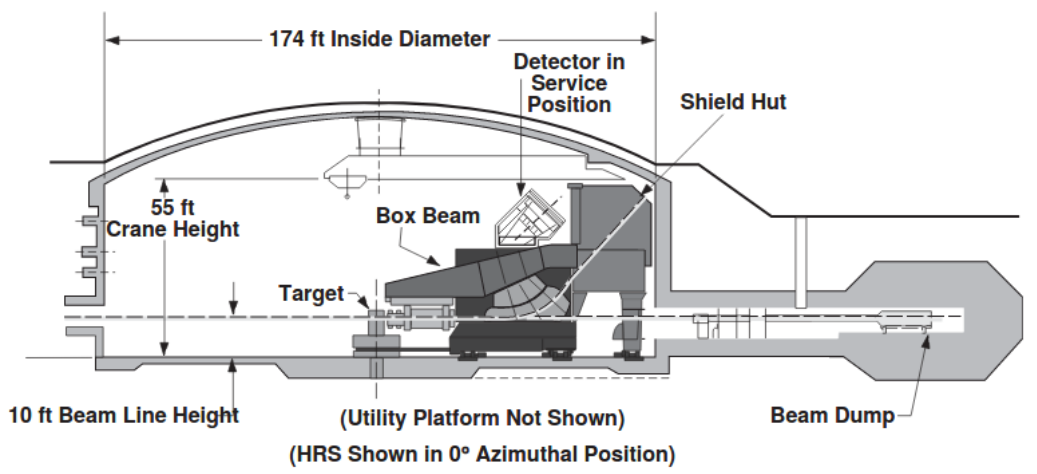

Figure 2-3: Side view of Hall A. Figure 2 from [32]. 


\section{$2.3 \quad$ Hall A Beamline}

The beam exits the accelerator after acceleration. Then it is deflected by an arc of eight dipole magnets to enter the experimental Hall A (see Fig. 2-5). Then it goes through several beamline instruments (e.g. beam position monitor, beam current monitor, raster...) to hit the target chamber where the electron-nuclei scattering takes place. Depending on the cross section of allowed processes, part of the incident electrons are scattered off the target, collected by one of the two spectrometers, and transported to the detector hut by a series of magnets. The rest of beam electrons continue to travel downstream to the beam dump. Fig. 2-4 provides an overview of in-hall instruments during this experiment.

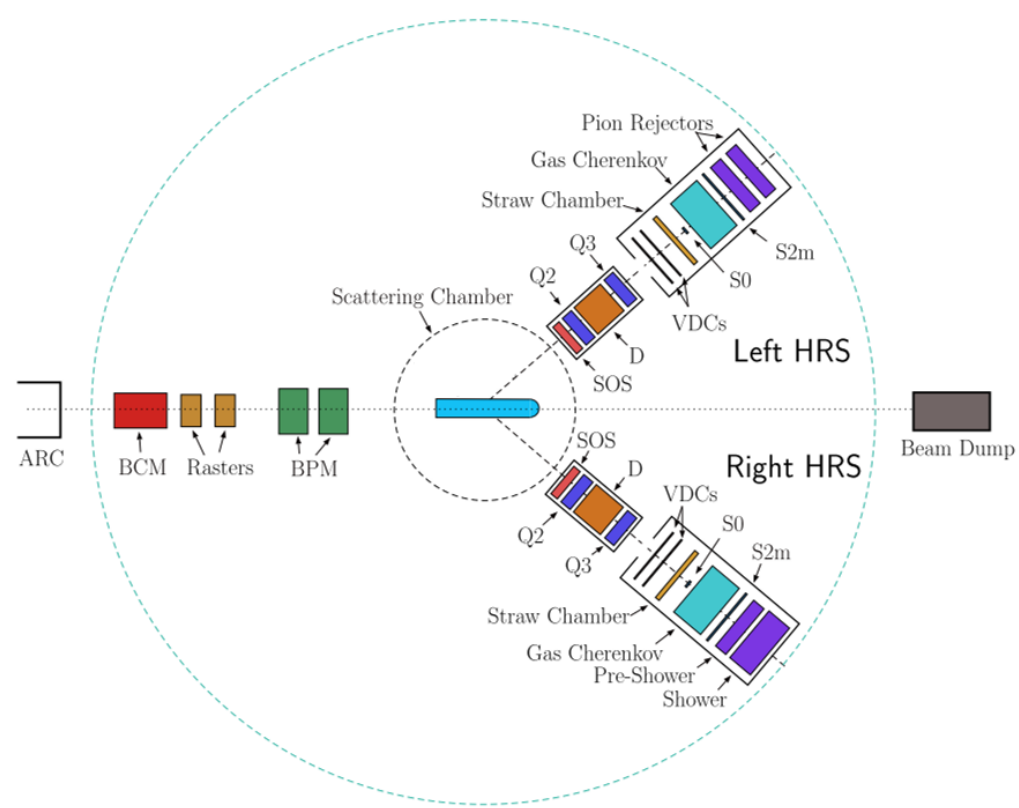

Figure 2-4: Schematic drawing of Hall A during E12-11-112 experiment. Adapted from [33]. 


\subsubsection{Arc and Beam Energy Measurement}

The Machine Control Center (MCC) constantly monitors the beam energy in the the accelerator. The actual beam energy delivered to the hall is calculated from the bending angle (from position measured by SuperHarps on the entrance and exit of the arc) and the magnetic field (see the "arc method" in [32]). It provides a correction factor on the MCC reported beam energy, see Table 2.3.1 [34]. The actual beam energy is provided to the Hall A analysis software (called "Analyzer", see Sec. 3.1) for the vertex reconstruction.

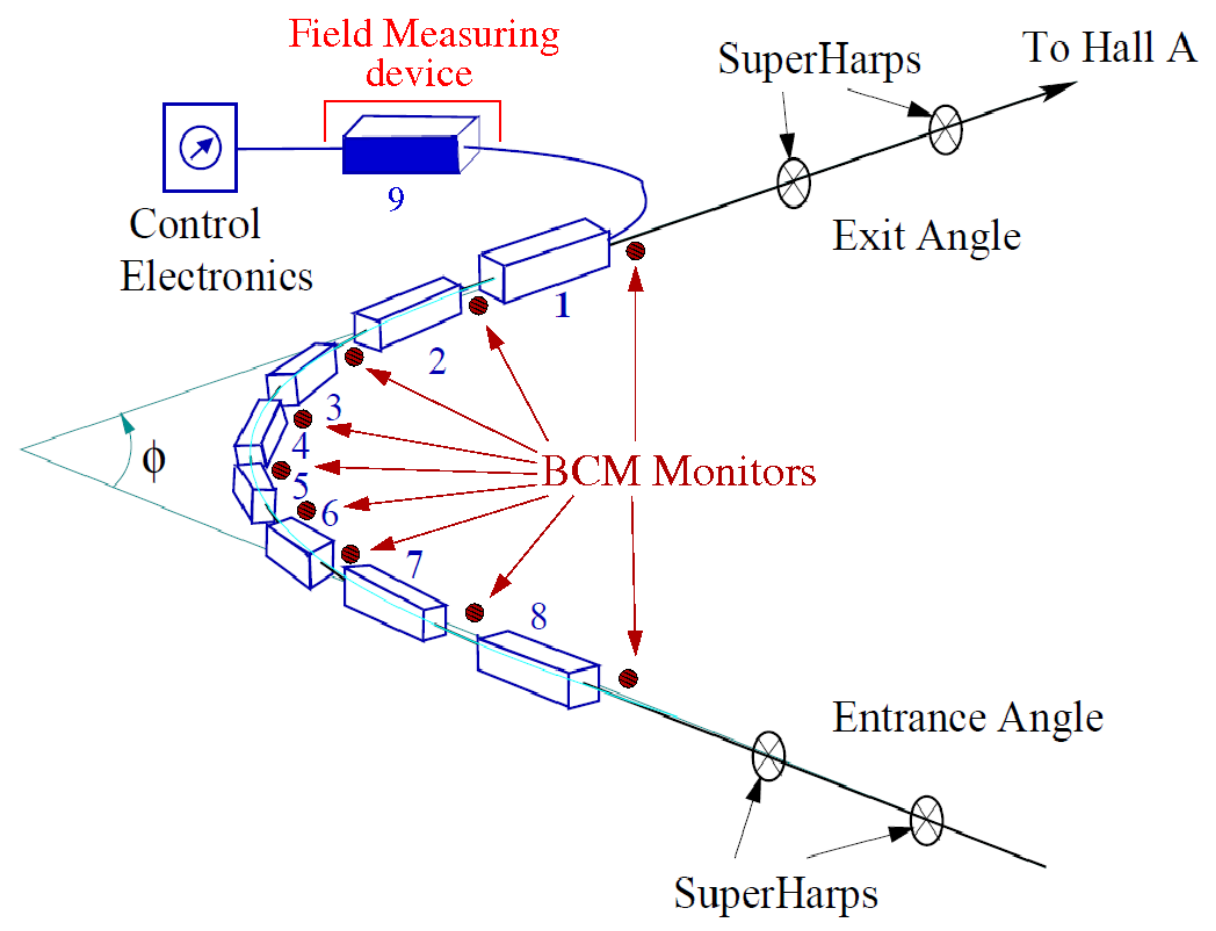

Figure 2-5: Drawing of the arc section of the Hall A beam line from [35]. 


\begin{tabular}{llll}
\hline Passes & Correction Factor & Beam Energy (MCC) & Beam Energy (Hall A) \\
\hline 2 & $1.0025(5)$ & $4318.5 \mathrm{MeV}$ & $4329.5 \mathrm{MeV}$ \\
\hline 1 & $1.0016(5)$ & $2218.5 \mathrm{MeV}$ & $2222.1 \mathrm{MeV}$ \\
\hline 0.5 & $1.0025(5)$ & $1168.5 \mathrm{MeV}$ & $1171.4 \mathrm{MeV}$ \\
\hline
\end{tabular}

Table 2.2: Energy correction factors for different beam passes from [34].

\subsubsection{Beam Current measurement}

About $25 \mathrm{~m}$ upstream of the target chamber along the beamline, two Beam Current Monitor(BCM) cavities and a Parametric Current Transformer (PCT) called UNSER [36] are installed in a thermal-isolated box (see Fig. 2-6). Each BCM cavity (uptream and downstream) is tuned to the beam frequency $(1497 \mathrm{MHz})$. Its responding signal, whose strength is proportional to the beam current, goes through a down-converter and turns into a $10 \mathrm{kHz}$ signal for accurate amplitude measurements [37]. The cavity signal after down-converter is split and sent to one of the four receivers (three analog and one digital) where the amplitude is converted into a voltage level. Then the DC signal goes through a Voltage-to-Frequency converter, and eventually is recorded by a scaler in the detector hut. Since the analog receiver has a limit range of linearity, three multiple modules with different gain settings $(\mathrm{x} 1, \mathrm{x} 3$, or $\mathrm{x} 10)$ are used in combination with the three analog receivers to fully cover the beam current range from $2 \mu \mathrm{A}$ to $100 \mu \mathrm{A}$. Within their linearity ranges, all receivers from upstream and downstream cavities should provide consistent beam current values after calibration. A pair of digital receivers were installed and tested during the $G_{M}^{p}$ experiment. Each digital receiver has a linearity range of $1 \mu \mathrm{A}$ to $100 \mu \mathrm{A}$.

The scaler rates from BCM cavities are linearly correlated with the beam current. To determine the gain factor, BCM signals are calibrated against the absolute beam current which is measured by UNSER [36]. Similar to BCM signals, the UNSER signal is recorded by the scaler as a frequency proportional to the measured beam current. Its gain is stable 


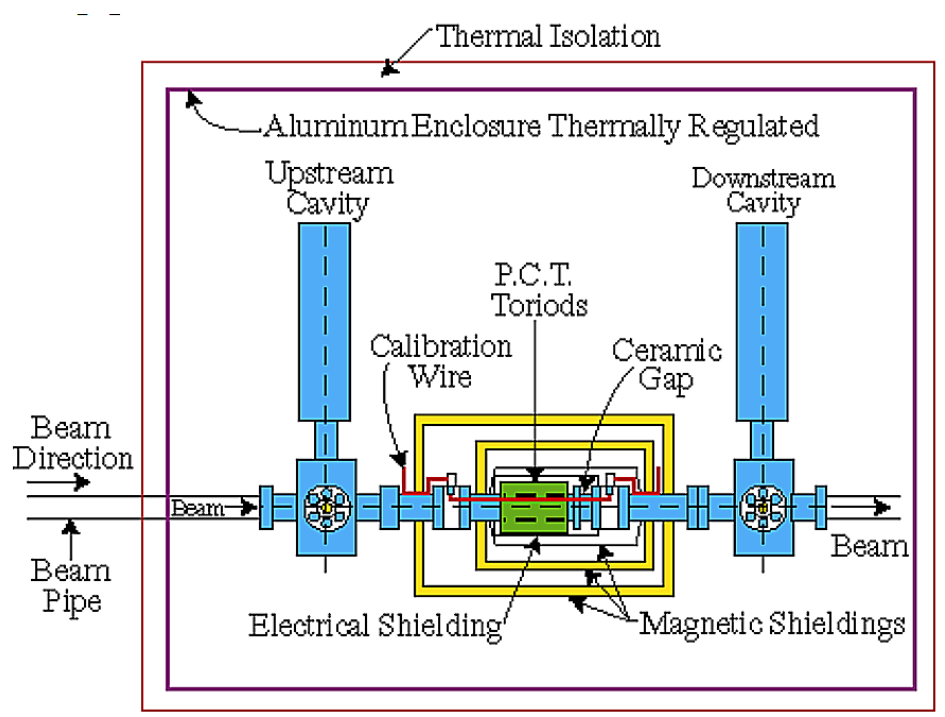

Figure 2-6: Schematics of beam current instruments from Ref. [37].

and accurate to $0.1 \%$ [38, but the zero offset drifts over time and is sensity to temperature. The first step of BCM calibration procedure is to calibrate UNSER against a known current source. A series of currents $(2,5, \ldots, 30 \mu \mathrm{A})$ are sent to UNSER through the calibration wire. A linear fit between the source current and the UNSER frequency were performed to find its offset and gain (see Fig. 2-7).

$$
I_{\mathrm{UNSER}}=\text { off } \text { set }_{\mathrm{UNSER}}-\text { gain }_{\mathrm{UNSER}} \cdot \text { freq }_{\mathrm{UNSER}}
$$

Then instead of the wire current, a series of beams are delivered to the Hall. And another linear fit between the UNSER measured current and the BCM frequency was used to decide the BCM offsets and gains (see Fig. 2-8). In this analysis, we used the downstream digital receiver signals (dnew) to calculate beam current, that is:

$$
I_{\mathrm{BCM}}(\mu \mathrm{A})=-0.084( \pm 0.103)+3.300( \pm 0.028) \times 10^{4} \cdot \text { freq }_{\text {dnew }}(\mathrm{Hz})
$$



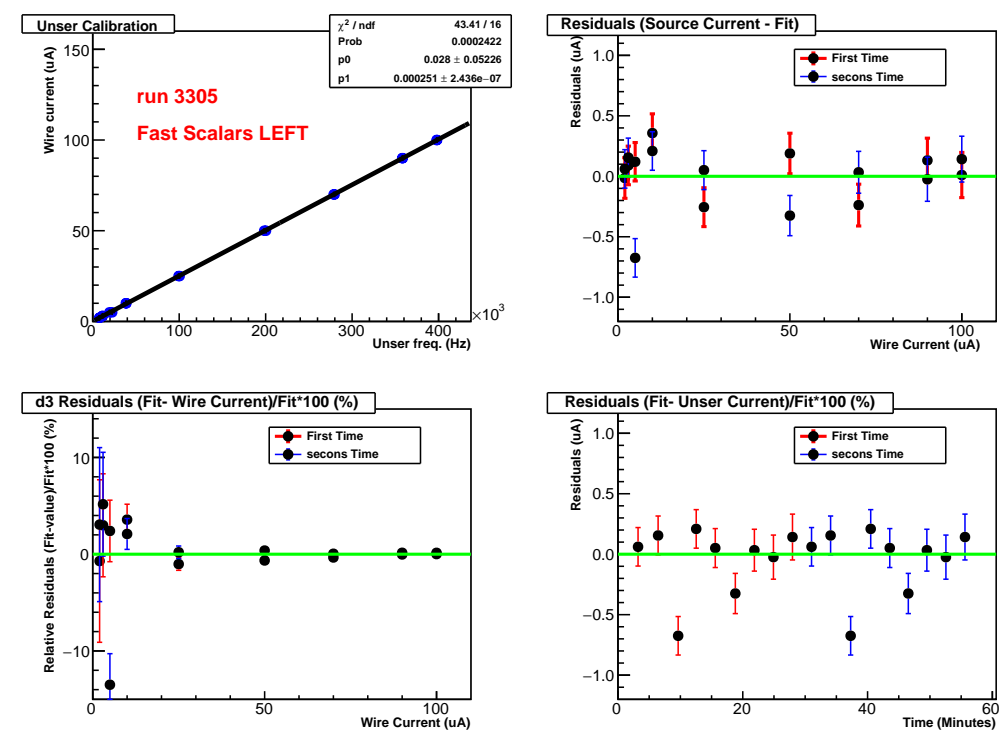

Figure 2-7: A linear fit of UNSER frequency against the wire current, and the residual test. Figure taken from [39].

With the $22.5 \mu \mathrm{A}$ production beam current, the uncertainty of this calibration is $\sim 0.22 \mu \mathrm{A}$.

\subsubsection{Beam Position}

In the Hall Coordinate System (HCS), $z$ is the beam direction, $x$ pointing to the beam left, and $y$ vertical up. The hall center (also the ideal target position) is defined as $z=0$. Alone the beamline and upstream to the target chamber, several instruments are installed to measure the beam position, including a pair of Beam Position Monitors (BPM) to monitor the realtime beam position, two Harp scanners to measure beam position intrusively, and two pairs of raster magnets to raster the beam (see Fig. 2-9p.

\section{Beam Position Measurement}

Two beam position monitors, IPM1H04A and IPM1H04E, are located in the upstream of the target. One BPM chamber contains four antennas. Signals from antennas are converted to 

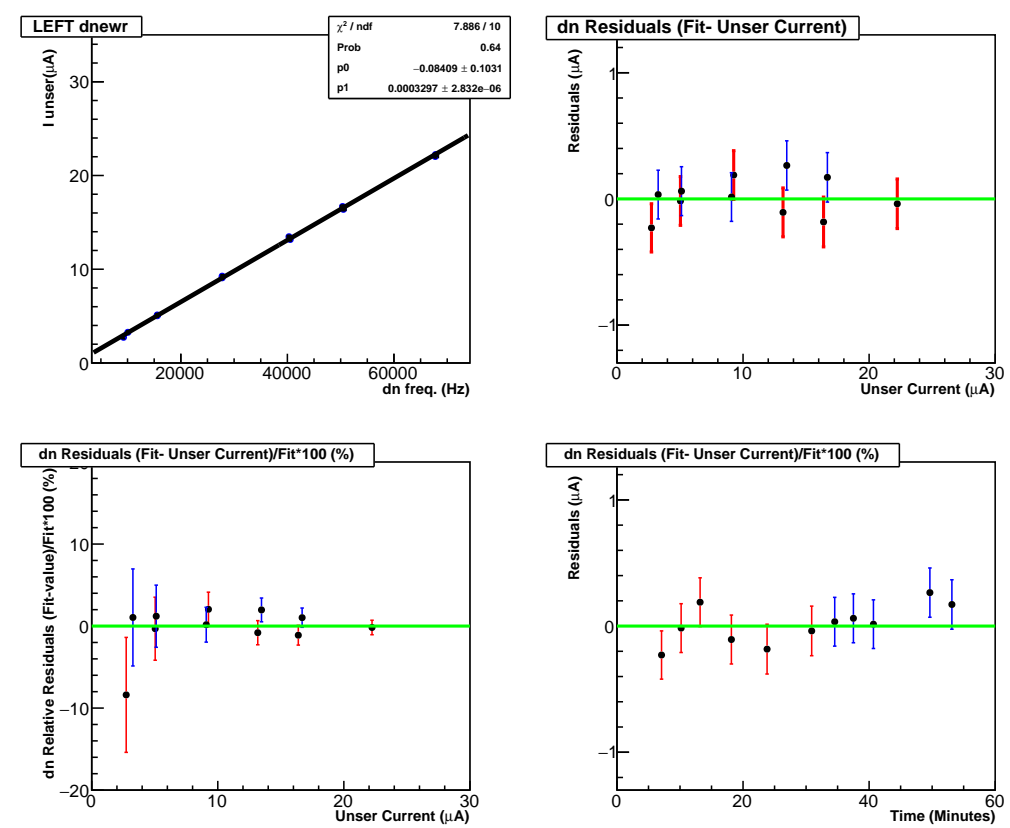

Figure 2-8: Linear fit of the UNSER-measured beam current against the BCM dnew frequency from [40].

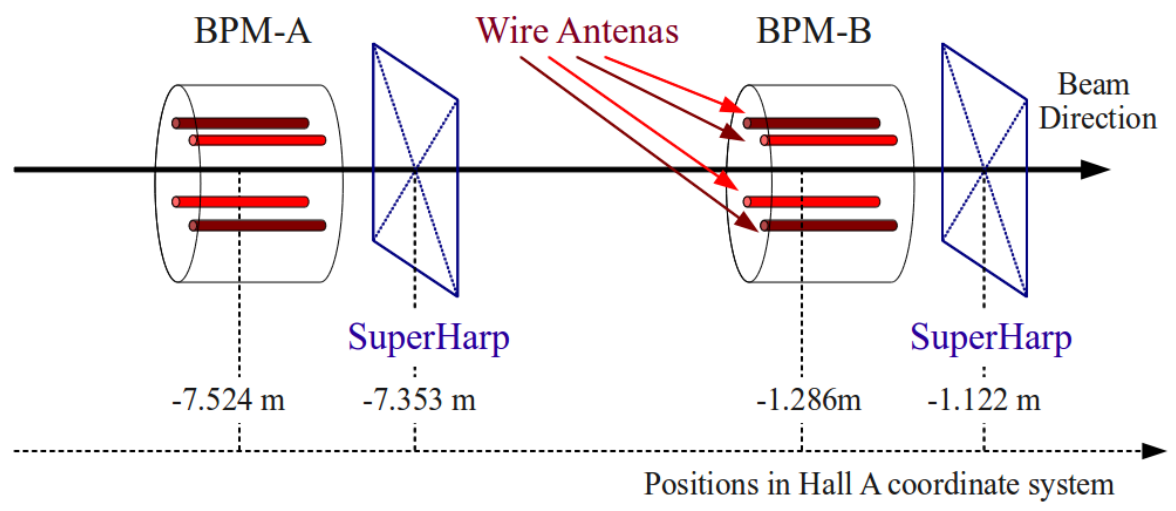

Figure 2-9: BPMs and Harps position in Hall Coordinate System. Figure 3.7 from [35]

DC levels proportional to the signal strength, and recorded by an analog-to-digital converter (ADC) in each detector hut. Each pair of signals from diagonal antennas are used to calculate the relative position the beam along that direction. To obtain the absolute beam position, the BPM signals are calibrated against the beam position from a Harp scanner (IHA1H04A/B) next to it. In a dedicated Harp scan run, the Harp is inserted into the 
beamline transversely to let the three wires (as shown in Fig. 2-11) interact with beam electrons. Position and charge from the wire are recorded to determine the beam position on the Harp plane [41]. Meanwhile the BPM signals are recorded. This process is repeated several times with different beam position (so-called "Bull's eye scan") to map out the conversion matrix between BPM signals and beam position at Harp position [42]. The the Harp is moved away from beam for production runs.
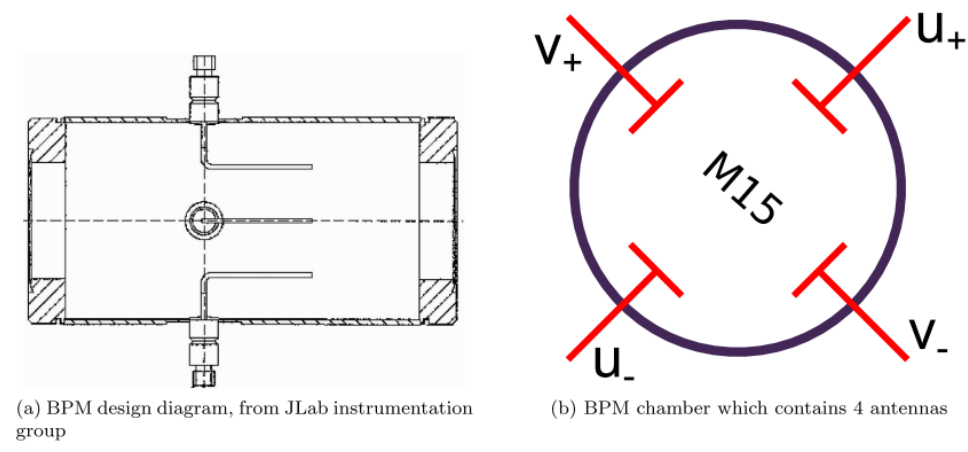

Figure 2-10: Diagrams of the BPM chamber from [43].

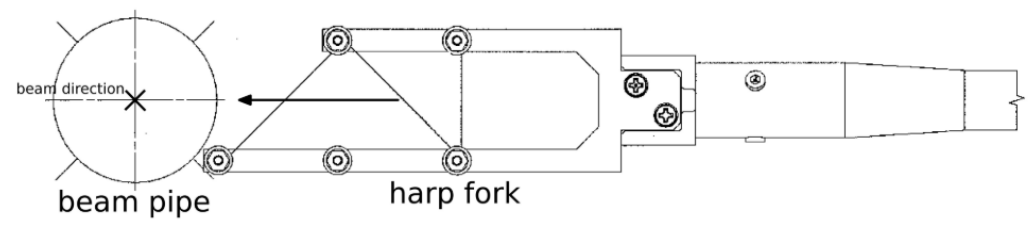

Figure 2-11: The harp scanner. [43].

\section{Raster}

The typical beam spot size is a few hundred micrometer. To minimize the local damage of a target from high intensity beam, two pairs of raster magnets were installed on the upstream of BPMs to distribute the beam in a $2 \mathrm{~mm} \times 2 \mathrm{~mm}$ square on target. Each 

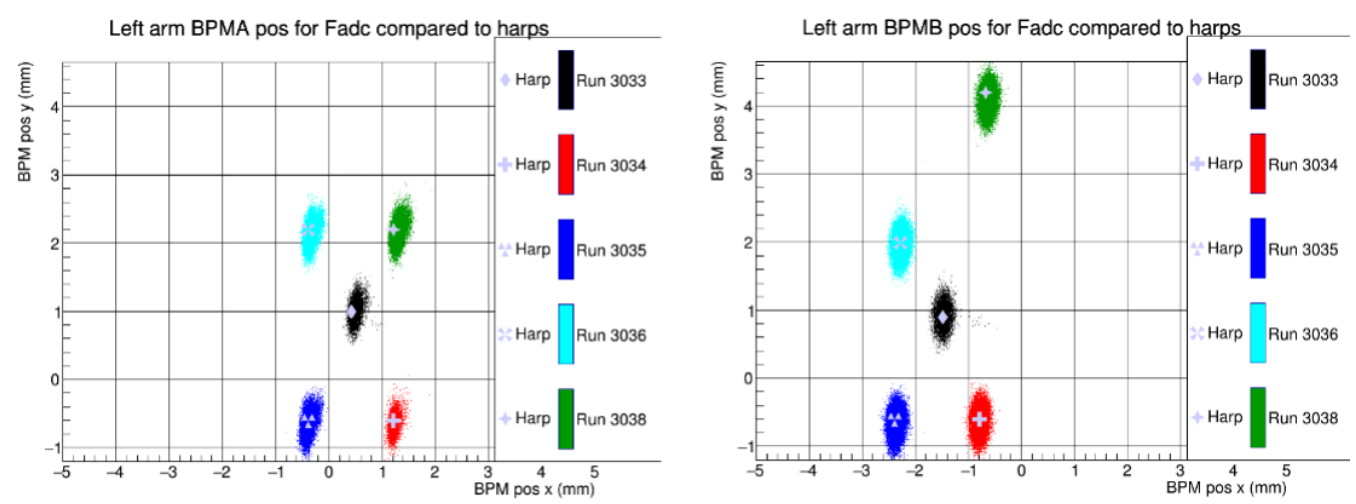

Figure 2-12: Beam positions read from the calibrated BPMs comparing to Harp scan results [42].

raster contains two dipole magnets to bend the electron beam in vertical and horizontal directions in $\mathrm{a} \sim 25 \mathrm{kHz}$ triangular waveform. The two rasters were designed to work in sync to provide enough bending for up to $12 \mathrm{GeV}$ beam. On October 142018 the raster A (relative upstream) failed. So part of L17-SRC2 data were taken with raster B only. The raster current was recorded as a $\mathrm{DC}$ level in $\mathrm{ADC}$. The beam position displacement from the center is linearly dependent on the raster current magnitude.

Since the BPM readback is significantly delayed comparing to the raster frequency (see Fig. 2-15), it can not provide instantaneous position of a rastered beam, but still gives the average position correctly. Then for a give event, the real-time beam position relative to the beam center is reconstructed from the raster's driven current (phase lag between the raster signal and the beam on target pattern is ignored). Each time the beam condition changed, a dedicated calibration run with a $2 \mathrm{~mm} \times 2 \mathrm{~mm}$ carbon hole target was taken to check the center and size of the rastered beam. See [44 for details. 


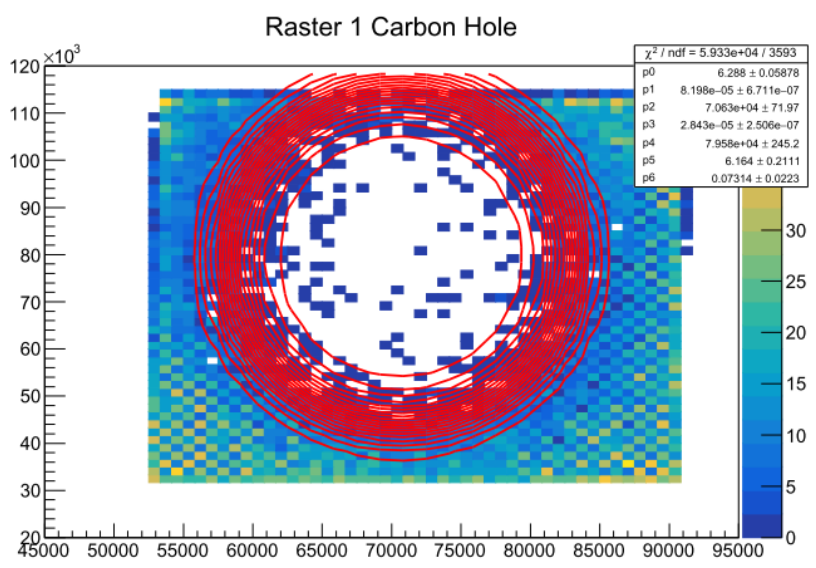

Figure 2-13: The reconstructed reaction vertex distribution on the target plane with the carbon hole target. The hole in the center has a known diameter of $2 \mathrm{~mm}$. The ratio between the fitted diameter from the plot and the actual hole diameter provides a scaling factor for the raster calibration. Figure taken from [44].

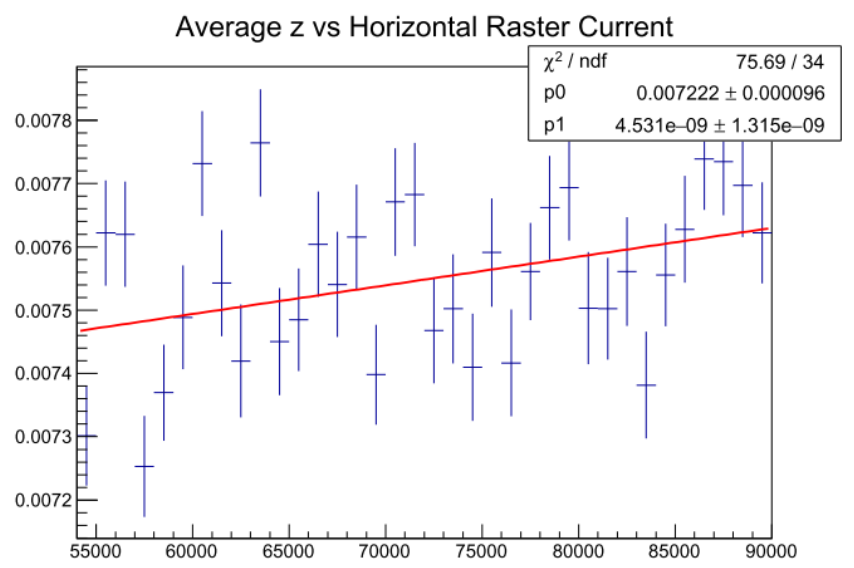

Figure 2-14: A linear fit of the raster current with respect to the reconstructed vertex $z$ position. The slope is 0 within uncertainties, which indicates no correlation between the raster current and the reconstructed vertex z position, as it supposed to be. Figure taken from [44.

\subsection{The Target System}

The upstream beam pipe is terminated with a $0.2 \mathrm{~mm}$ Be window inside the Bigbite scattering chamber [45]. The chamber has an inner radius of 41in. The target ladder sits in the center of the chamber (and the hall). As shown in Fig. 2-16, five identical target cells, and 


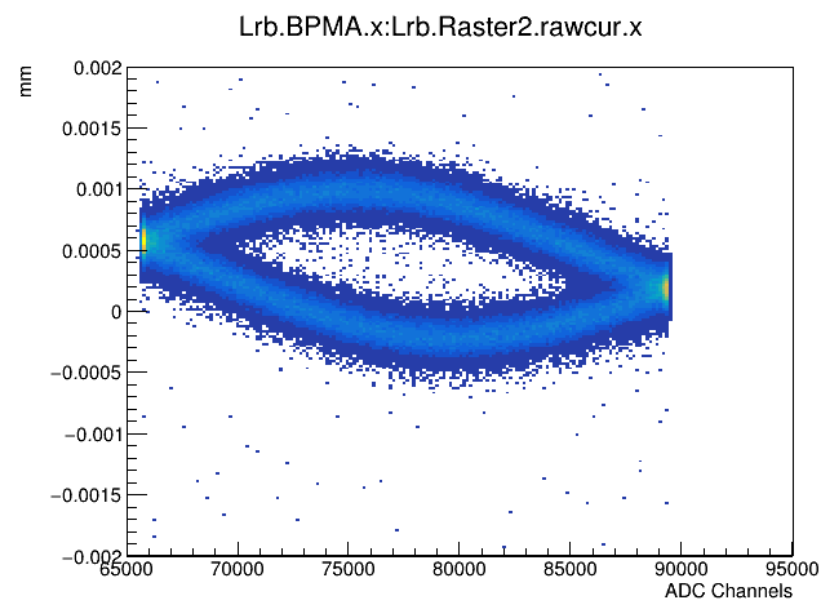

Figure 2-15: Phase lag between the BPM-measured beam position and the raster current.

also several foil targets were installed in the ladder during all tritium experiments. A target encoder system is used to move the entire ladder up and down to make sure the beam go through the center of a chosen target.

\subsubsection{Gas Targets}

All Tritium group experiments shared a unique design of low pressure gas target system to provide an acceptable tritium gas area density with minimal risk from tritium handling. The $25 \mathrm{~cm}$ long, $1.25 \mathrm{~cm}$ in diameter sealed cell can hold $1000 \mathrm{Ci}(0.1 \mathrm{~g})$ of Tritium gas. There were four target cells (tritium, deuterium, hydrogen, helium-3) in the target ladder. One additional empty target cell was installed for background study. All cells were fabricated locally with Alloy $7050\left(2.81 \mathrm{~g} / \mathrm{cm}^{3}\right)$ which has high thermal conductivity. The endcap thickness of each target was carefully measured. Then one empty cell were sent to Savannah River Site for tritium filling in October 2017. Since tritium slowly decays into helium-3, the original tritium cell was sent back to Savannah River after the Spring 2018 run period 


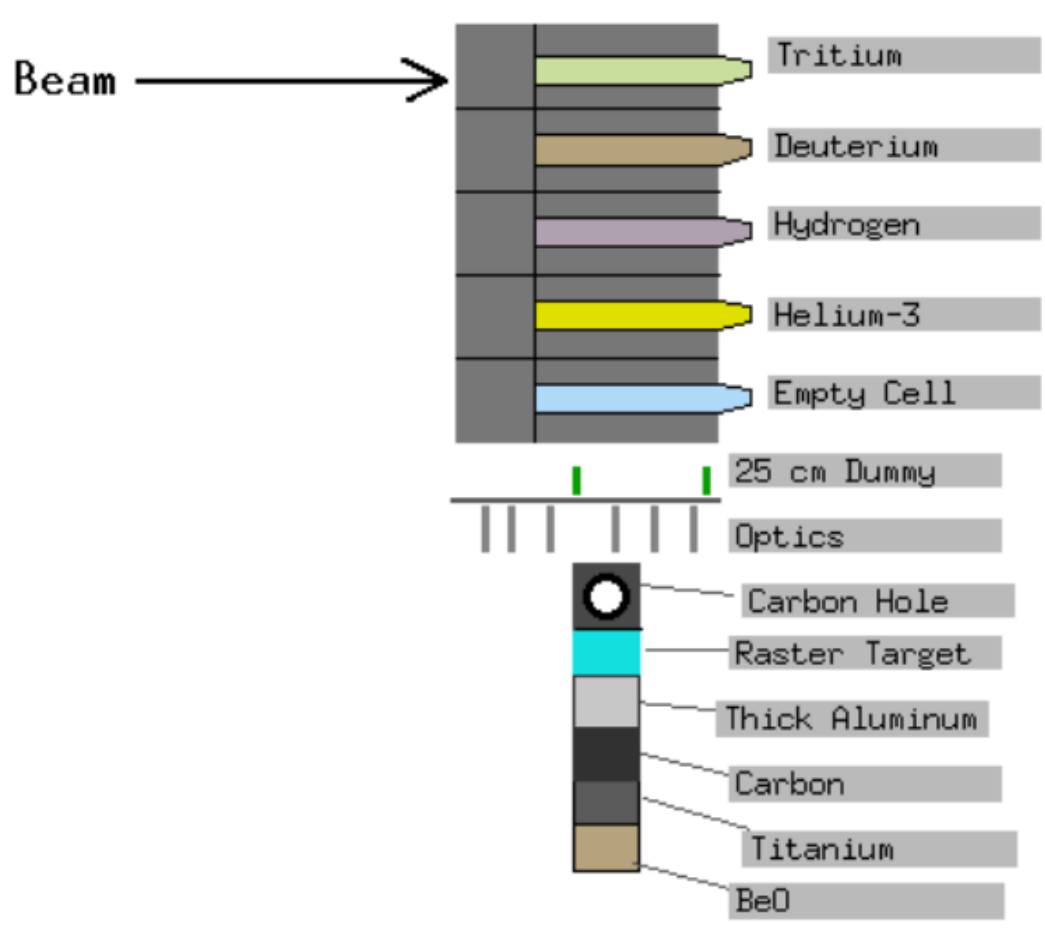

Figure 2-16: A diagram of target ladder from the target control GUI.

and replaced by a brand new tritium target. The second tritium cell had a hydrogen contamination of unknown source. See Sec. 4.2.3. Other gas cells were filled at Jefferson Lab. Details of each target are listed in Table 4.2 .3 .

The maximum allowable beam current on all gas cells is set at $22.5 \mu \mathrm{A}$ to avoid cell damage from beam.

\subsubsection{Solid Targets}

Several solid targets were installed for various purposes. The Dummy target is two pieces of aluminum that are aligned with the entrance and exit windows of the gas target cell respectively. Each foil is five times as thick as the entrance window. It was used as a replacement 


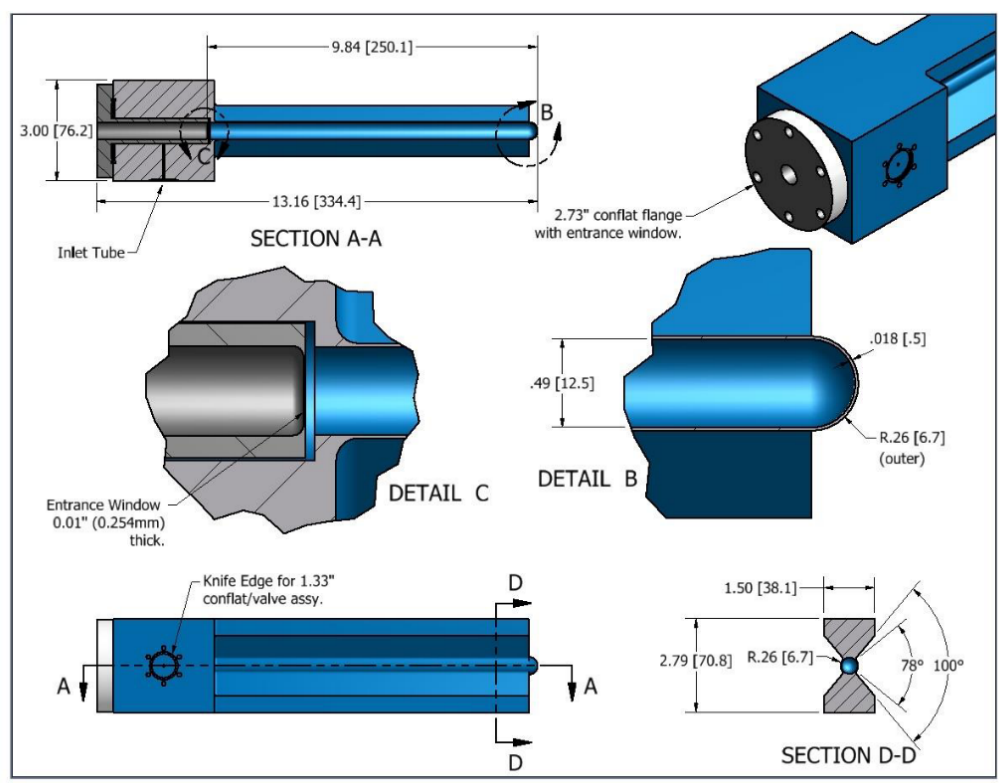

Figure 2-17: An engineer drawing of target design details.

for the empty cell at low rate kinematics, that is, L17-SRC2, EP-PK, and EP-SRC.

The optics target is eleven $0.25 \mathrm{~mm}$ carbon foils that are evenly spaced at $2.5 \mathrm{~cm}$ to cover the entire target cell length. it is designed for optics calibration (see Sec. 3.2.1). The carbon hole target is a carbon foil that has a $2 \mathrm{~mm}$ diameter hole in the center. It is used for beam centering and also raster calibration.

The single foil carbon target is used for pointing study (see Sec. 3.2.1). It provides production data on Carbon that can be used to study SRC ratios as well. We also took production data on the titanium target which was recycled from the Argon experiment [47]. Cross section analysis with solid targets is not included in this thesis.

\subsection{High Resolution Spectrometers}

Two identical High Resolution Spectrometers are designed to reach up to $4 \mathrm{GeV}$ central momentum with $2 \times 10^{-4}$ momentum resolution and 2 mrad transverse angular resolution. 


\begin{tabular}{|l|lllllll|}
\hline Target & Type & $\mathbf{A}$ & $\mathbf{Z}$ & Amu & $\begin{array}{l}\text { Thickness } \\
\left(\mathrm{g} / \mathrm{cm}^{2}\right)\end{array}$ & $\begin{array}{l}\text { Entrance } \\
\text { Window } \\
(\mathrm{mm})\end{array}$ & $\begin{array}{l}\text { Exit Window } \\
(\mathrm{mm})\end{array}$ \\
\hline Tritium (1st) & gas & 3 & 1 & 3.016 & $0.0851 \pm 0.0008$ & $0.208 \pm 0.004$ & $0.343 \pm 0.047$ \\
\hline Tritium (2nd) & gas & 3 & 1 & 3.016 & $0.0851 \pm 0.0008$ & $0.257 \pm 0.021$ & $0.276 \pm 0.021$ \\
\hline Deuterium & gas & 2 & 1 & 2.014 & $0.1422 \pm 0.0008$ & $0.215 \pm 0.004$ & $0.294 \pm 0.056$ \\
\hline Hydrogen & gas & 1 & 1 & 1.01 & $0.0708 \pm 0.0004$ & $0.311 \pm 0.001$ & $0.330 \pm 0.063$ \\
\hline Helium-3 & gas & 3 & 2 & 3.016 & $0.0534 \pm 0.0006$ & $0.203 \pm 0.007$ & $0.328 \pm 0.041$ \\
\hline Empty Cell & solid & 27 & 13 & 26.98 & N/A & $0.254 \pm 0.0051$ & $0.279 \pm 0.0051$ \\
\hline Dummy & solid & 27 & 13 & 26.98 & N/A & $1.248 \pm 0.002$ & $1.248 \pm 0.002$ \\
\hline Thick Aluminum & solid & 27 & 13 & 26.98 & $1.37 \pm 0.007$ & N/A & N/A \\
\hline Carbon(single foil) & solid & 12 & 6 & 12.01 & $0.0883 \pm 0.0002$ & N/A & N/A \\
\hline Titanium & solid & 48 & 22 & 47.876 & $0.4081 \pm 0.0008$ & N/A & N/A \\
\hline
\end{tabular}

Table 2.3: Target thickness from [46]. Note that the entrance window thickness of the 1st tritium cell is updated from 0.253 to 0.208 as suggested by Dave Meekins. The 2nd tritium cell thickness shown here didn't include the hydrogen contamination (see section 4.2 .3 for details.

The HRS entrance is $1.2 \mathrm{~m}$ away from the hall center with $6 \mathrm{msr}$ solid angle coverage.

Particles inside the HRS are transported through two quadrapoles (Q1 focuses in vertical direction, Q2 focuses in horizontal direction), a 45 degree vertical bending Dipole, then another quadrapole Q3 to reach the focal plane. The pathlength is $25.7 \mathrm{~m}$. The layout of HRS is shown in Fig. 2-18, More information on the HRS design characteristics is listed in Table 2-19.

Fig. 2-20 shows the probability of electron events going through the Left HRS to reach the focal plane as a function of in-plane angle $\phi_{t g}$ and out-of-plane angle $\theta_{t g}$ at various momentum range. $\delta_{t g}$ is the deviation of the particle momentum from the HRS central momentum in fraction. In the momentum range $-4.0 \%<\delta_{t g}<4.0 \%$, almost $100 \%$ of events within the nominal HRS angular acceptance:

- in-plane angle (horizontal): $-30 \mathrm{mrad}<\phi_{t g}<30 \mathrm{mrad}$,

- out-of-plane angle (vertical): $-60 \mathrm{mrad}<\theta_{t g}<60 \mathrm{mrad}$,

were successfully transported to the focal plane. This group of $\delta_{t g}, \phi_{t g}, \theta_{t g}$ ranges is widely 
used in Hall A cross section ratio analysis, hereinafter referred to as the "loose cuts."

The operating currents of this QQDQ combination of magnets were tuned to achieve best HRS resolutions. The setting is expected to scale with the HRS central momentum. However, the original superconducting Q1 on both HRSs were replaced by the iron magnets from the Hall C Short Orbit Spectrometer [48] during the $G_{M}^{p}$ experiment. [33]. The new Q1 magnets lost its linearity between the driven current and the magnetic field once go above $3 \mathrm{GeV}$. This is in particular a challenge to our SRC kinematics taken with Left HRS. We compensated this saturation by sending higher-than-default driven current to Q1. A current scan was performed with optics target to find the best settings [49]. But the Q1 focus, hence the entire transport matrix of the HRS would not be the same as the standard one even with the optimized settings. Detailed optics study was performed for each kinematics with momentum setting higher than $3 \mathrm{GeV}$, see Sec. 3.2.1.

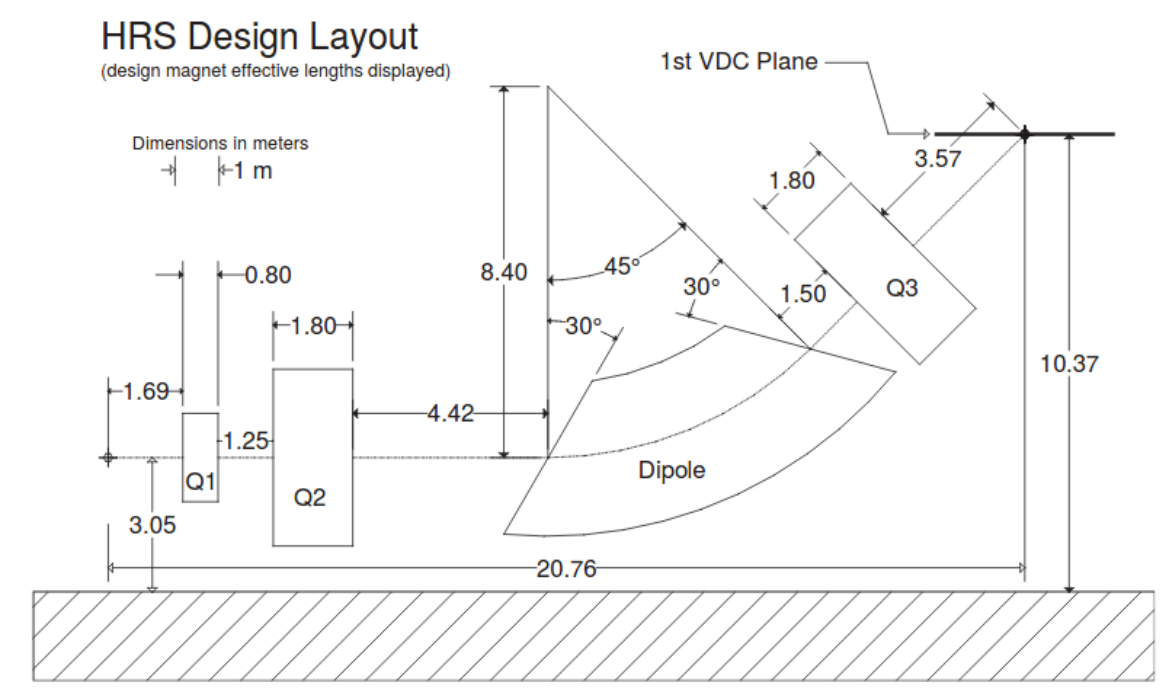

Figure 2-18: HRS layout. Figure 5 from [32]. 
Main design characteristics of the Hall A high resolution spectrometers; the resolution values are for the FWHM

\begin{tabular}{ll}
\hline Configuration & $\mathrm{QQD}_{n} \mathrm{Q}$ vertical bend \\
Bending angle & $45^{\circ}$ \\
Optical length & $23.4 \mathrm{~m}$ \\
Momentum range & $0.3-4.0 \mathrm{GeV} / c$ \\
Momentum acceptance & $-4.5^{\circ}<\delta \mathrm{p} / \mathrm{p}<+4.5^{\circ} \%$ \\
Momentum resolution & $1 \times 10^{-4}$ \\
Dispersion at the focus $(D)$ & $12.4 \mathrm{~m}$ \\
Radial linear magnification $(M)$ & -2.5 \\
$D / M$ & 5.0 \\
Angular range & $12.5-150^{\circ}$ \\
$\quad$ HRS-L & $12.5-130^{\circ}$ \\
$\quad$ HRS-R & $\pm 30 \mathrm{mrad}$ \\
Angular acceptance & $\pm 60 \mathrm{mrad}$ \\
$\quad$ Horizontal & \\
$\quad$ Vertical & $0.5 \mathrm{mrad}$ \\
Angular resolution & $1.0 \mathrm{mrad}$ \\
$\quad$ Horizontal & $6 \mathrm{msr}$ \\
$\quad$ Vertical & $\pm 5 \mathrm{~cm}$ \\
Solid angle at $\delta \mathrm{p} / \mathrm{p}=0, y_{0}=0$ & $1 \mathrm{~mm}$ \\
Transverse length acceptance & \\
Transverse position resolution & \\
\hline
\end{tabular}

Figure 2-19: HRS design characteristics. Table 1 from [32].

\subsection{Detector Package and Triggers}

The central ray of scattered electrons exiting LHRS Q3 are detected by the a series of detectors (see Fig. 2-21). This experiment used the standard LHRS detector package to measure the electron track and momentum. This detector package includes two layers of Vertical Drift Chambers (VDCs) for tracking, two layers of scintillators (S0 and S2) to determine the time-of-flight and generate trigger signals, a $\mathrm{CO}_{2}$ gas Cherenkov Chamber as a threshold detector for pion/electron separation, and two layers of Pion Rejectors (PR1 and PR2) to measure the electron energy deposit. 

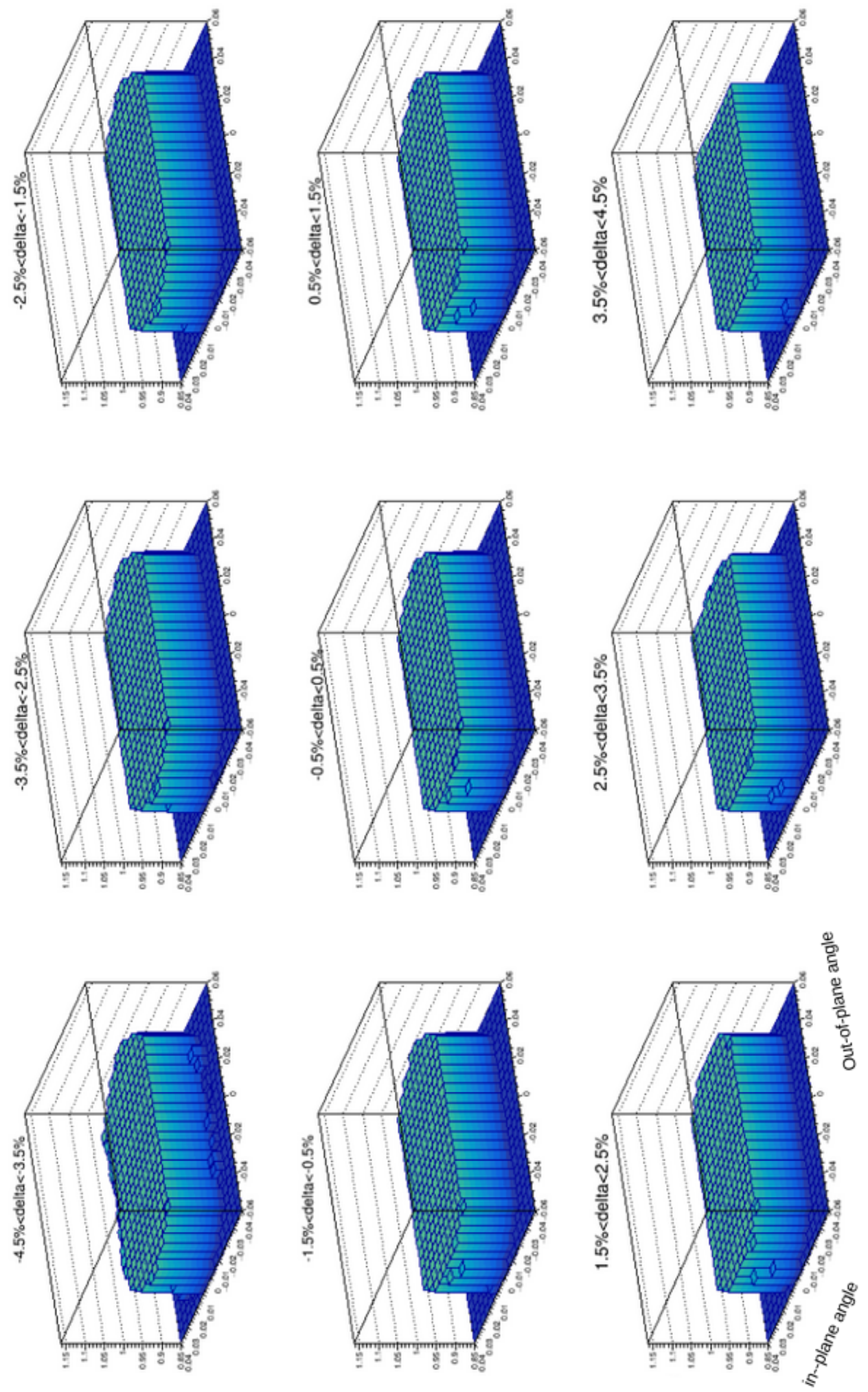

Figure 2-20: LHRS acceptance from the Hall A Monte-Carlo simulation (see Sec. 4.3) as a function of in-plane angle $\left(-0.04 \mathrm{rad}<\phi_{t g}<0.04 \mathrm{rad}\right)$ and out-of-plane angle $(-0.06$ $\left.\operatorname{rad}<\theta_{t g}<0.06 \mathrm{rad}\right)$ at L17-PK. Each subplot covers $1 \%$ of $\delta_{t g}$ range. 


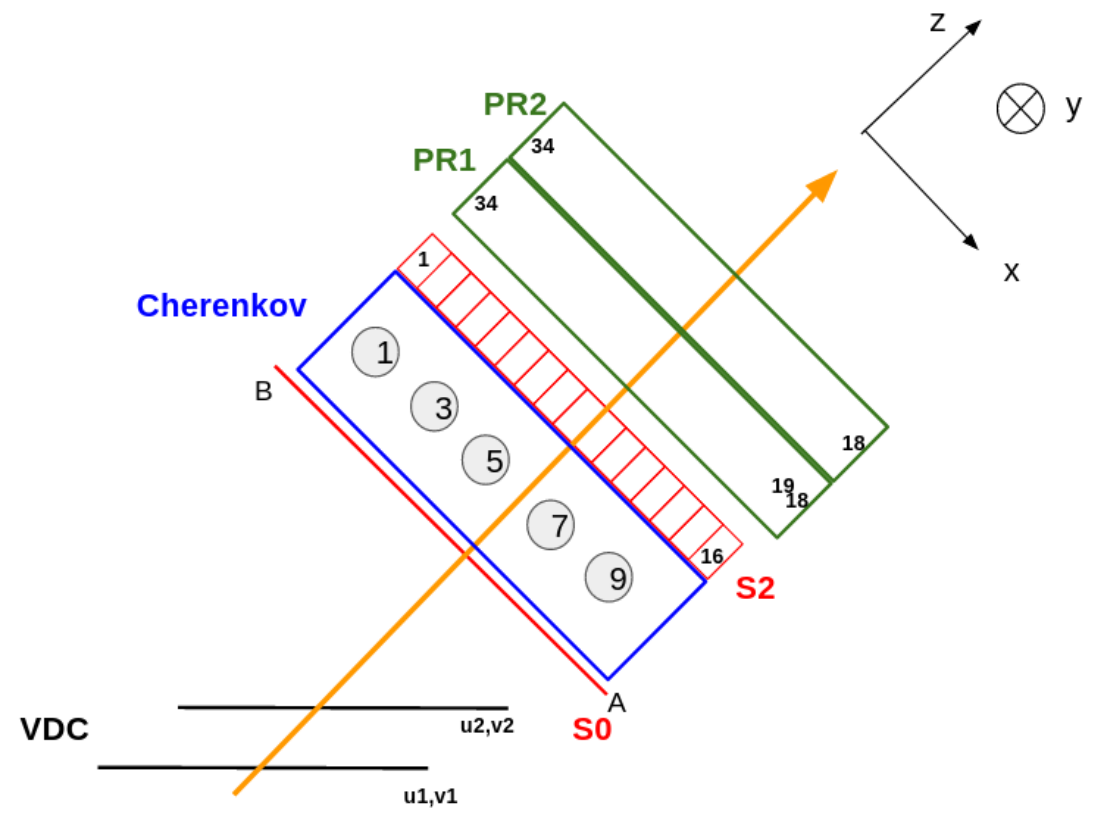

Figure 2-21: Schematic drawing of Left HRS detector package. Letters and numbers correspond to each PMT signal as is indexed in Analyzer.

\subsubsection{VDCs}

Two vertical drift chambers are installed parallel to the ground level. Each chamber is 26 mm thick, filled with 50-50 Argon-Ethane gas (by volume) [50]. The top and bottom panel of each chamber is operating with $-3.5 \mathrm{kV}$ high voltage, in the middle are two groups of 368 sense wires with ground potential. The scattered particle going through VDCs creates ionized electrons along its track. Those ionized electrons drift in the electric field with a drift velocity of $50 \mu \mathrm{m} / \mathrm{ns}$ toward sense wires. The drift time of ionized electrons is converted to the drift distance for the track reconstruction. One electron track may trigger 3 to 7 adjacent wires. As shown in Fig. 2-23, a typical VDC cluster with five wires fired is reconstructed from their timing information to obtain the track intercept with the wire plane. The track reconstruction algorithm in Analyzer takes one cluster per VDC plane to fit a linear trajectory. If a plane has more than one cluster, Analyzer goes over all possible 
combinations of clusters (one from each plane) to find the group of four clusters with least $\chi^{2}$. That so-called "golden tarck" is store the reconstructed track in rootfiles with index 0 . This process is repeated with unused clusters and the result is stored with index $1,2, \ldots$ until no track can be reconstructed from unused clusters.

Fig. 2-24 shows the time spectrum for a given wire. The signals were recorded with time-to-

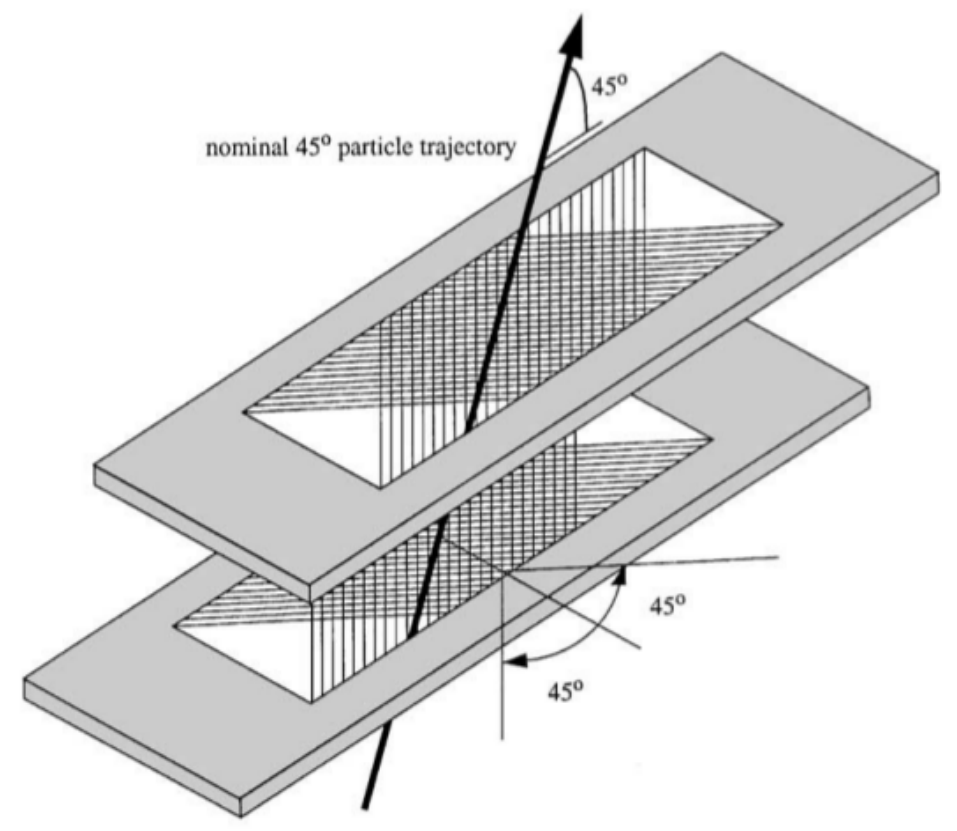

Figure 2-22: Schematic configuration of VDCs from [50.

digital converters (TDCs) in COMMON STOP mode so that the right edge of the spectrum corresponds to the shortest drift time with respect to the trigger signal. It is associated to electron tracks which go right through that wire. In the VDC calibration process this edge is identified for each wire as $t_{0}$. Then Analyzer can calculate the drift time a $t-t_{0}$. 


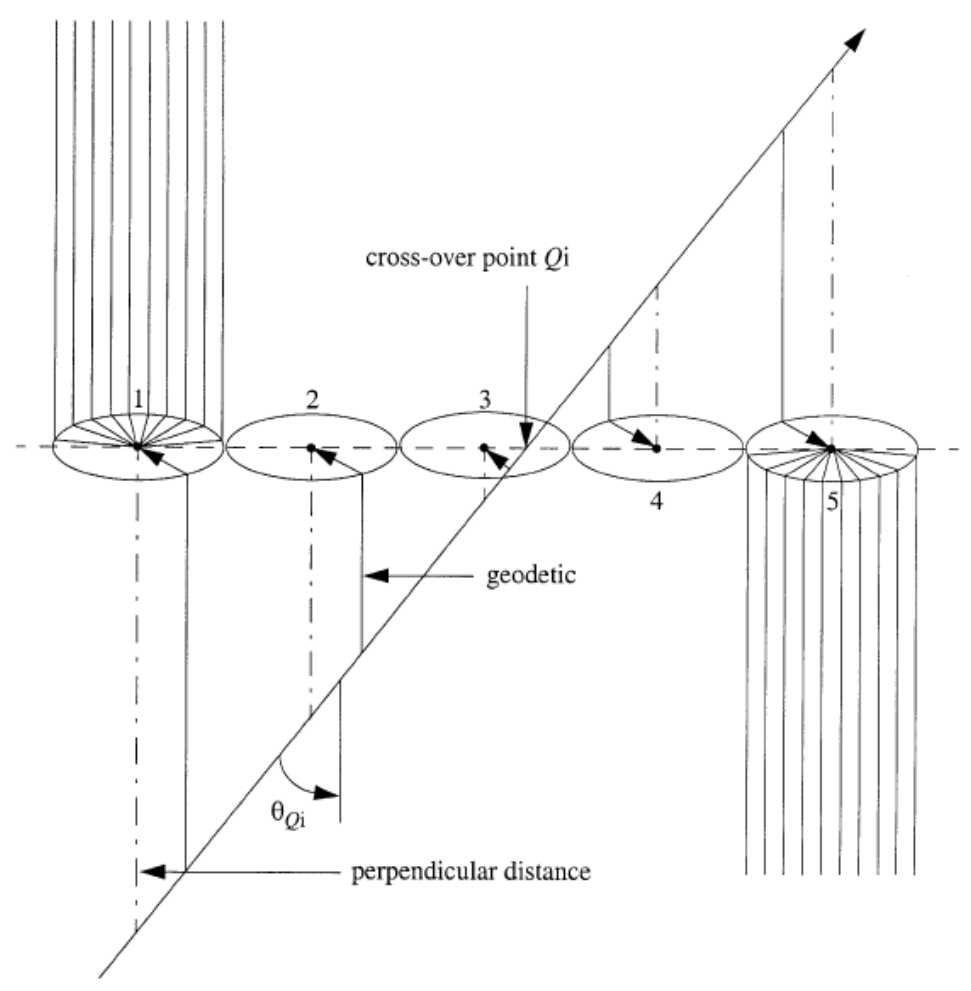

Figure 2-23: A typical 5-cell track event.

\subsubsection{Cherenkov}

The Cherenkov chamber is filled with $\mathrm{CO}_{2}$ gas. Its index of refraction is $n=1.00041$ which corresponds to the pion momentum threshold of Cherenkov radiation:

$$
\beta \geq \frac{1}{n} \Rightarrow p_{\min , \pi}=\frac{m_{\pi} c}{\sqrt{\frac{1}{\beta_{m i n, \pi}^{2}}-1}}=4.87 \mathrm{GeV} / \mathrm{c}
$$

while the threshold for electrons is only $17.85 \mathrm{MeV} / \mathrm{c}$. Electrons with momentum above the threshold go through the chamber and produce Cherenkov light. The light is reflected on 10 concave mirrors in the back of the chamber and collected by ten ET-9390KB Photomultiplier tubes(PMTs) on two sides of the detector. The acceptance range of each PMT is plotted in Fig. 2-26, During the $G_{M}^{p}$ experiment the LHRS Cherenkov chamber depth was 


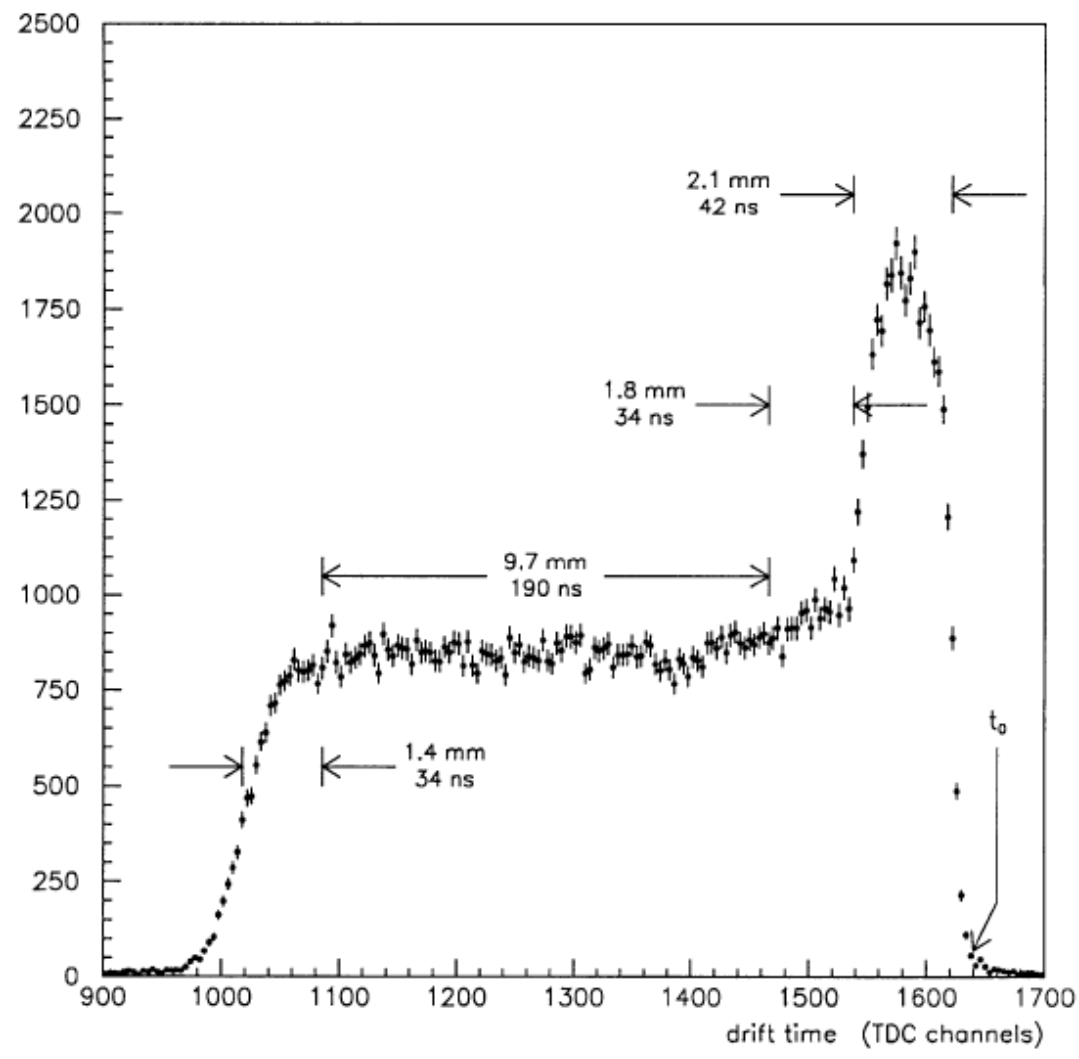

Figure 2-24: Time spectrum of a VDC wire from figure 17 in [50].

extended from $100 \mathrm{~cm}$ to $128 \mathrm{~cm}$ to increase the number of Cherenkov photons produced along the electron track. Also, a special wavelength shifting paint was applied on each PMT to improve the photon detection efficiency [51].

A raw ADC spectrum from one Cherenkov PMT is shown in Fig. 2-27. The ADC signal strength with respect to the pedestal level is proportional to the number of photons collected by that PMT. Next to the pedestal peak is a small Single Photon-electron Peak (SPE) created almost entirely by the "dark current" when the PMT emitted a single photon-electron and self-triggered. Contributions from low-charge background of thermoionic emission and the elastic scattering inside the PMTs are less than 10\% [51] and is irrelevant to this analysis. 

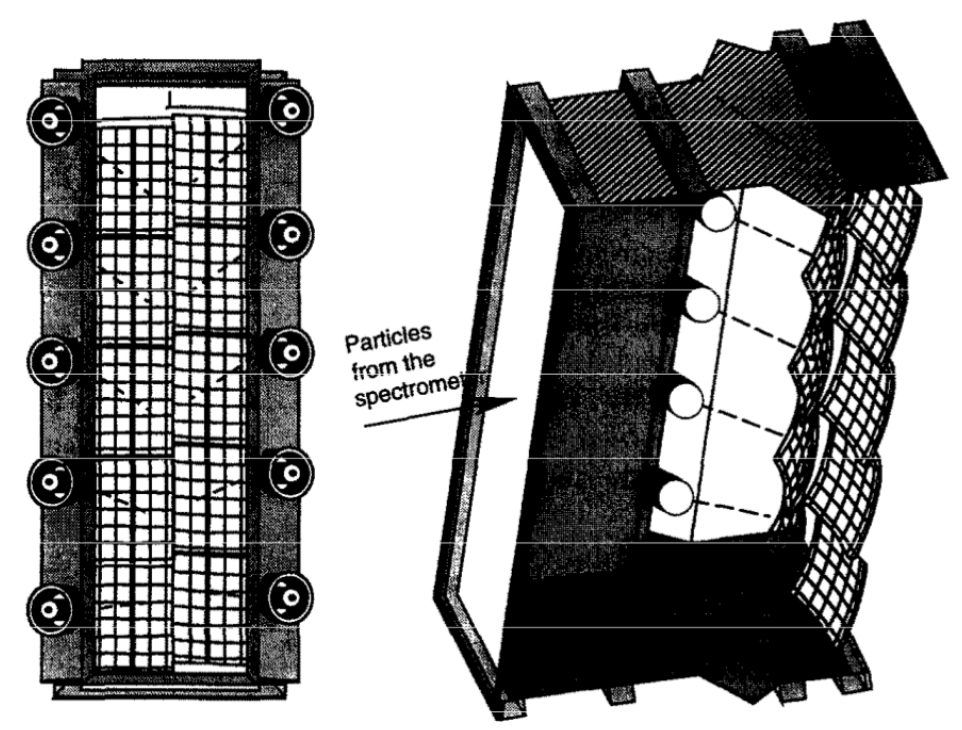

Figure 2-25: 3D and open view of Cherenkov from Ref. [52]. 10 curved mirrors on the back of Cherenkov chamber is used to redirect Cherenkov light to 10 PMTs (five on each side).

In the Cherenkov calibration process, the pedestal peak of each PMT was aligned to channel 0, and the SPE was scaled to channel 300. To check the performance of each PMT, we plotted the calibrated ADC spectrum ( see Fig. 2-28) and fitted it with a Poisson function. The fitted peak channel divided by 300 gives the average number of photons collected by that tube, which is around 15.

\subsubsection{Scintillators}

Two layers of plastic scintillators are installed before and after the Cherenkov counter to provide event timing information. In front of the Cherenkov is a $1.7 \mathrm{~m} \times 0.25 \mathrm{~m} \times 0.01 \mathrm{~m}$ single scintillator paddle S0. Each end of the paddle is connected to a PMT (S0A and S0B). The PMT signals are sent to both TDCs and ADCs. The S2 behind Cherenkov is a plane of 16 overlapped scintillator paddles.

The time-of-flight hence the speed of the particle $\beta$ can be calculated from the time differ- 


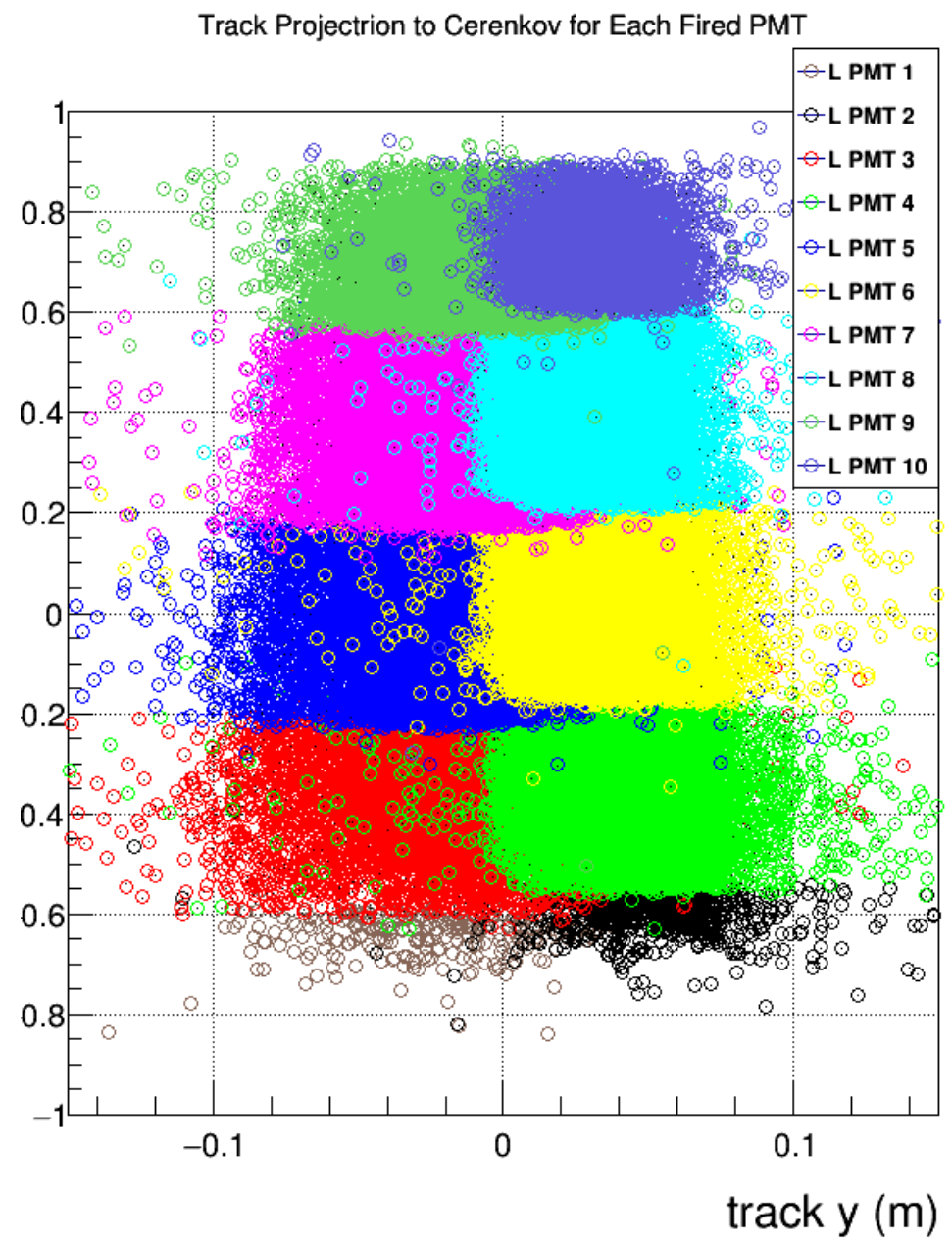

Figure 2-26: Tracks of events fired each PMT projected to the Cherenkov plane.

ence between two scintillator planes after corrected for the time-walk effect (by fitting the ADC and TDC signal correlations) and the signal travel time. In this analysis the particle identification had been good enough with the combination of Cherenkov and Pion Rejector signal cuts. So the scintillator signals were simply aligned such that the electron $\beta=1$ after the quality check.

\subsubsection{Pion Rejectors}

Two layers of lead-glass calorimeter blocks (PR1 and PR2) are located on the back of the detector package. Each layer contains 34 blocks as shown in Fig. 2-29. The particle goes 


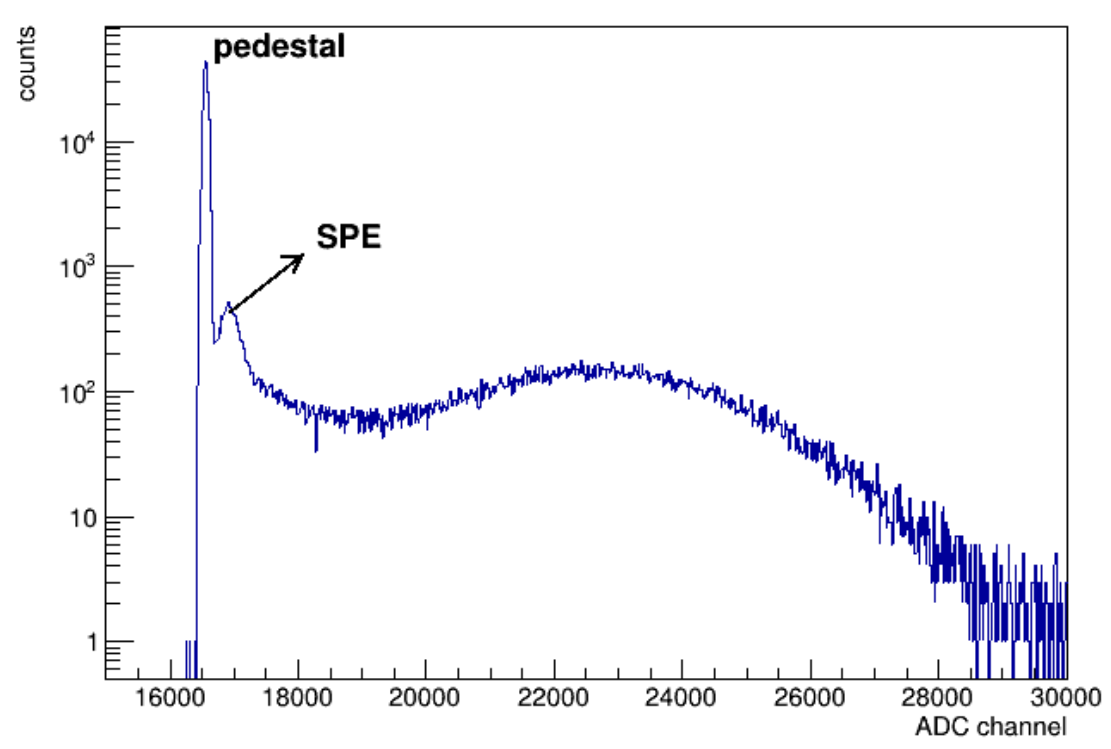

Figure 2-27: The Cherenkov PMT raw ADC spectrum.

through the pion rejectors and loses energy to create electron showers. In our kinematic settings the electrons are not likely to be fully stopped by the pion rejectors. But the sum of electron energy deposit in pion rejectors should be proportional to the electron energy. Fig. 2-30 shows that after calibration, the sum of PR1 and PR2 ADC signals of an electron event is not correlated with the the track position in general. Except at the large track $x$ (lower end of the detector) where the statistics are limited. Also, the ratio of deposit energy to the HRS central momentum centers at one. Note that among all fired lead-glass blocks, only those close to the particle track projections are included in the energy sum. Fig. 2-31 shows the correlation between the position of fired blocks and the electron track projections. 

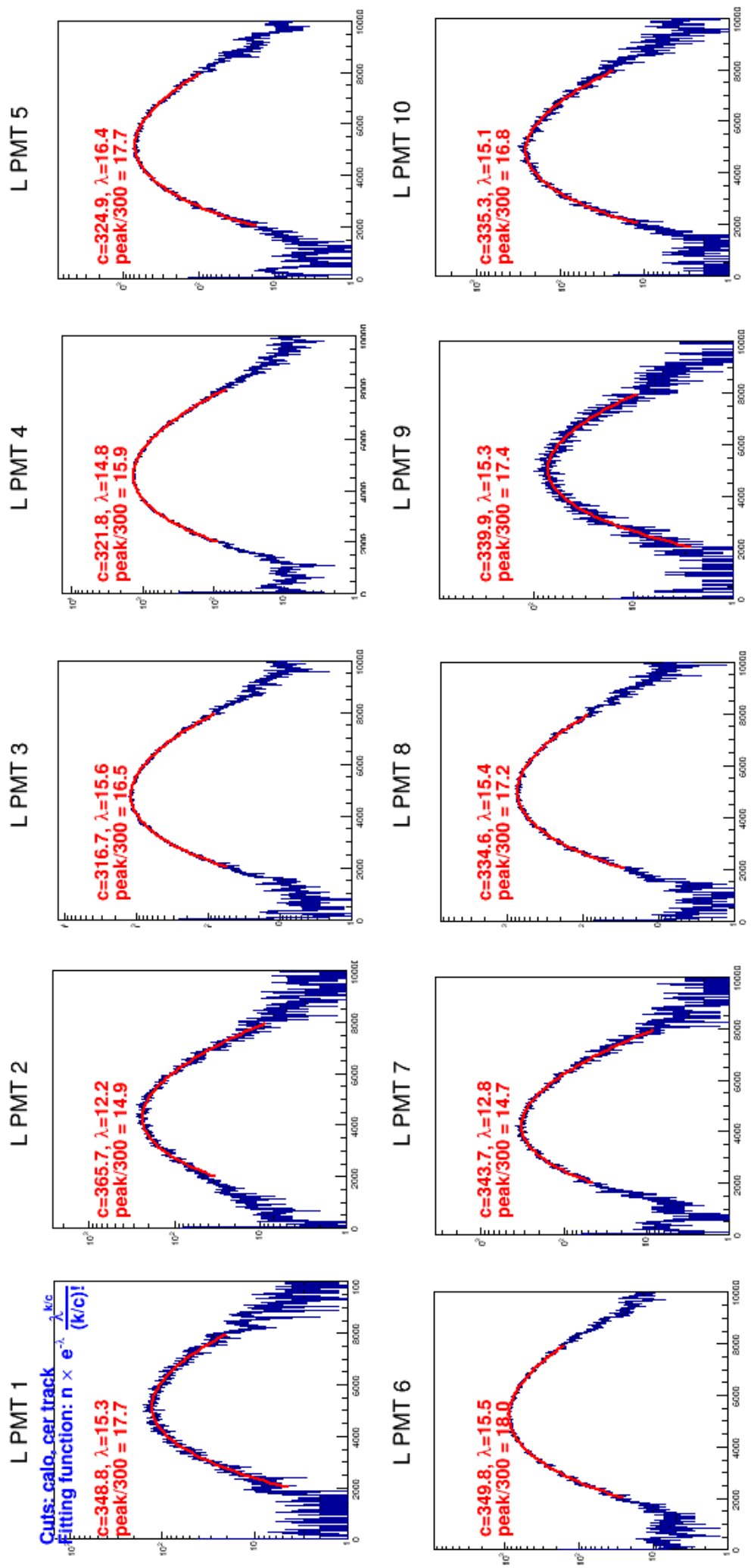

Figure 2-28: The Cherenkov PMT ADC spectrum after calibration. 


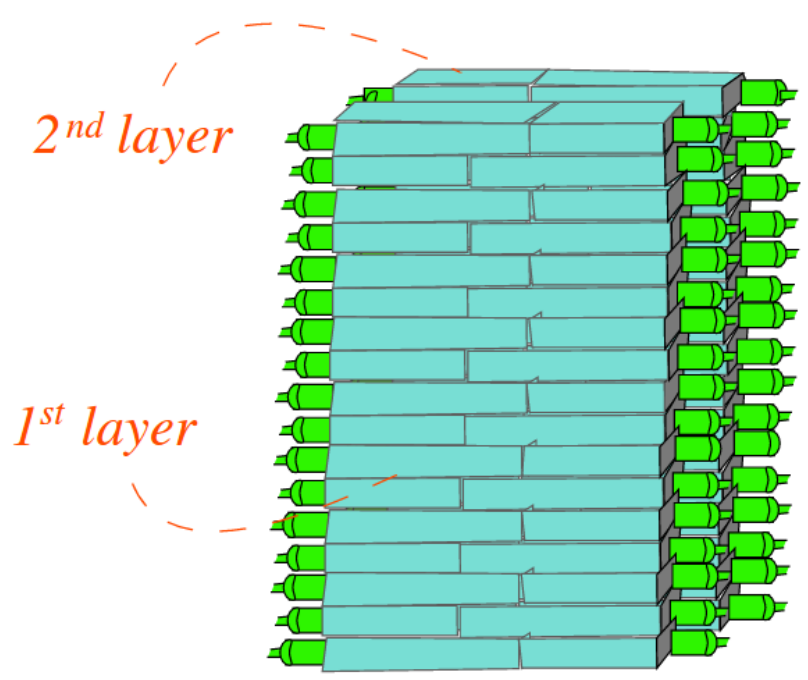

Pion Rejector

Figure 2-29: Schematic drawing of LHRS pion rejectors from [53].

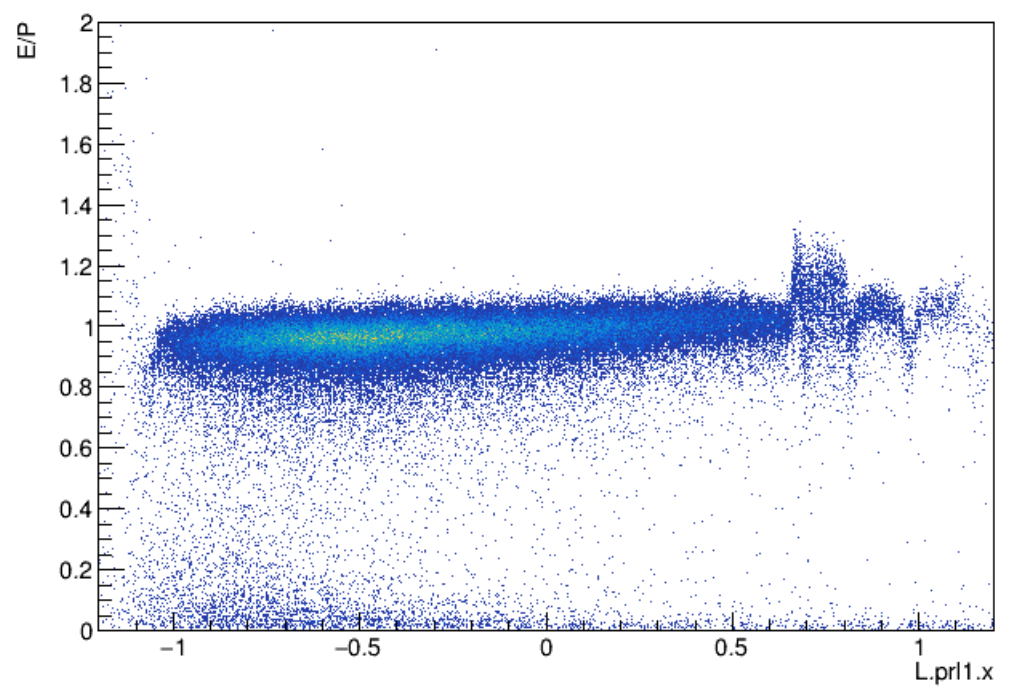

Figure 2-30: The sum of electron energy deposit in two layers of pion rejectors divided the track momentum is plotted with respect to the vertical projection of the track on the detector plane.

\subsubsection{Triggers}

The PMT signals from both sides of S0 paddle formed a logic "AND". For the S2 plane, those logic signals from each paddle were then combined through the fan-in/fan-out module 

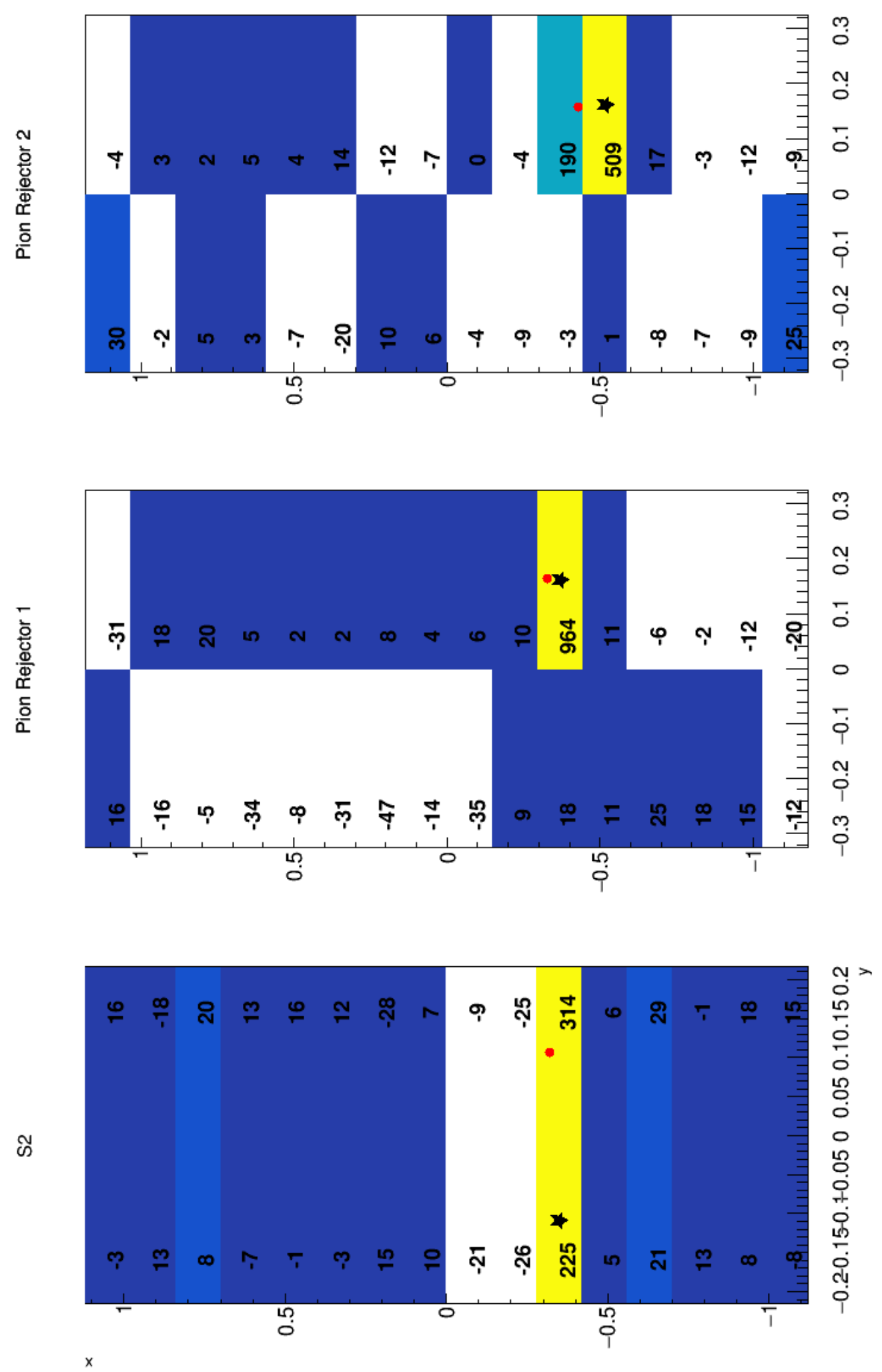

Figure 2-31: ADC singal amplitude from S2, PRL1, and PRL2 PMTs are plotted at each detector plane. Star Marker: the particle hit position estimated from ADC signals. Red Marker: the track projection on each detector plane. 
to generate the S2 trigger signal. Trigger signals from S0 and S2 were required to be in coincidence with the Cherenkov sum signal to form a production trigger. A schematic of trigger design is shown in Fig. 2-32, Besides the production electron trigger, two calibration triggers were built for the efficiency study. No re-timing module was used in this experiment. Those three triggers were manually adjusted so that the S2 signal would lead the time when it was fired.

- T1: S0 \& S2,

- T2: (S0 \& S2) \& Cer (production trigger),

- T3: (S0 || S2) \& Cer.

With this trigger configuration, we had $\tilde{1} 0 \mathrm{~Hz}$ T3 events and less than $1 \mathrm{~Hz} \mathrm{~T} 1$, T2 events in a background run without beam.

\subsection{Data Acquisition}

The ADC and TDC signals from detectors and beamline instruments were recorded once a trigger fired. Scaler counts and run conditions such as target temperature and HRS angle were inserted into the data stream every $4 \mathrm{~s}$ or 100 triggers. Every hour we stopped and restarted the the CEBAF Online Data Acquisition (CODA) software to created a new raw data file. Raw data collected during that period were stored as a "run" with a unique run number. The designated run number ranges are 3000 to 4200 for LHRS data, and 9000 to 9999 for RHRS data. The coincidence e'p data has a run number range of 10000 to 11000. During the experiment, we continuously monitored the beam quality, HRS and target status, and scaler rates to identify good production runs. Run-by-run information are stored in 
the Hall A Electronic Logbook as well as the Hall A MySQL database (halladb/tritonwork). A webpage interface of the database is maintained at https://hallaweb.jlab. org/experiment/Tritium/E12-11-112/tritium_page/runlist.php. 


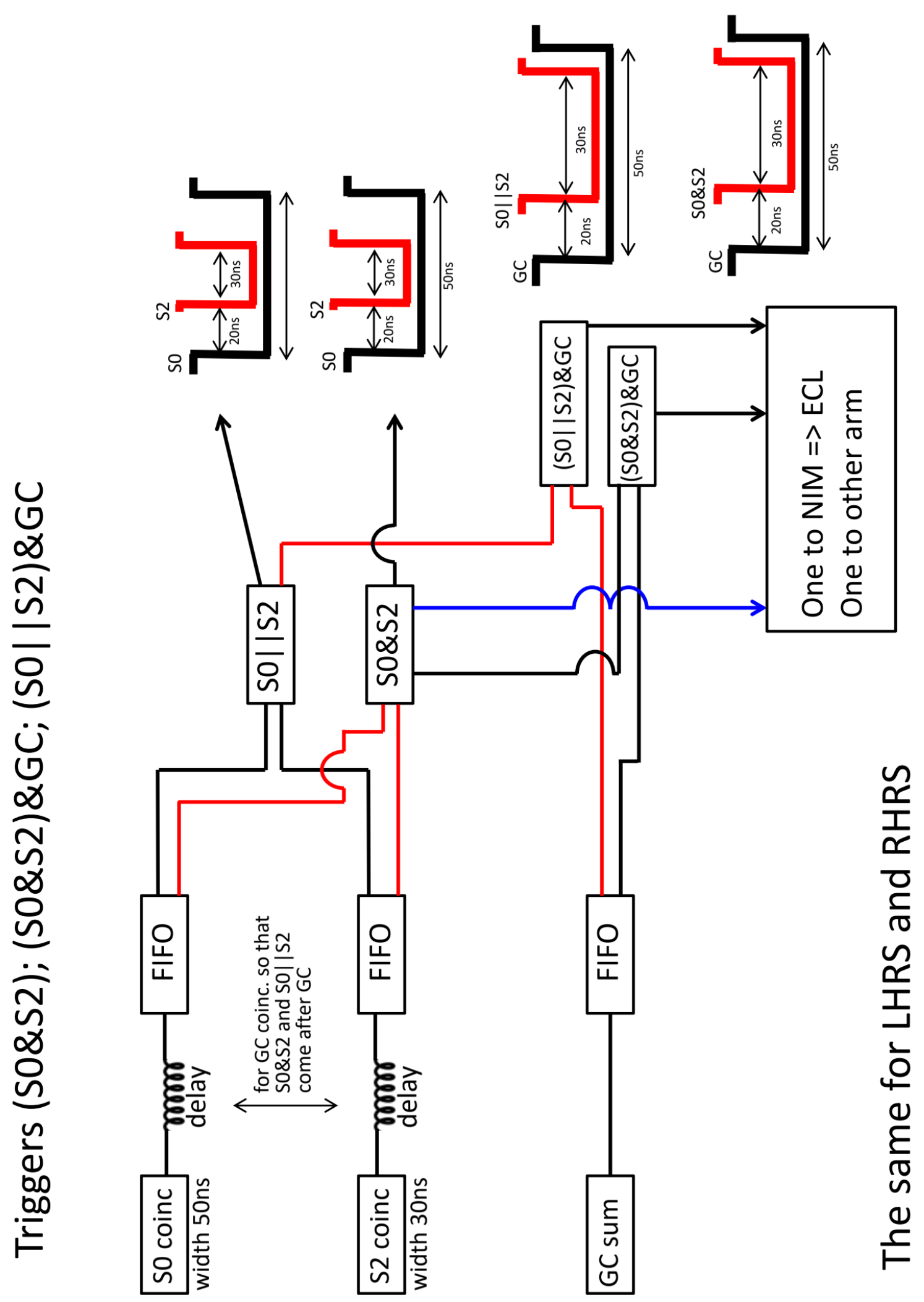

Figure 2-32: Schematics of LHRS triggers design by Florian Hauenstein. 


\section{Chapter 3}

\section{Data Analysis}

\subsection{Hall A Analyzer Software}

This data analysis was done with the standard Hall A physics analysis software (the "Analyzer"). Built on top of ROOT [54, this Analyzer decodes the detector signals from raw data, reconstructs reaction vertex, and writes results into rootfiles. The decoding process is called "replay". Proper replay scripts and a database with run conditions and instruments calibration information are necessary to a successful replay. A customized replay package for this experiment is maintained on GitHub [55].

\subsection{Event Reconstruction}

Every recorded event is reconstructed during the Analyzer decoding process. VDCs provide position $\left(x_{f p}, y_{f p}\right)$ and angle (in-plane angle $\phi_{f p}$, out-of-plane angle $\left.\theta_{f p}\right)$ information of a detected track at the focal plane. Those informations are used to reconstruct the scattered particle's track, which includes scattering angle (from $\phi_{t g}, \theta_{t g}$ ), the transverse vertex position as seen by HRS $\left(y_{t g}\right)$, and the scattered particle momentum $\left(\delta_{t g}\right)$, back to the target plane. Then the reaction vertex is reconstructed by taking the intercept of beam vector 
(from two BPMs) with the plane of the scattered particle track (from optics reconstruction). Ionization energy loss of the incoming and scattered particle is applied.

\subsubsection{Optics Calibration}

To first order, the four out of five target plane variables $(\delta, \theta, y, \phi)_{t g}$ are related to four focal plane variables $(x, \theta, y, \phi)_{f p}$ by the optics matrix

$$
\begin{aligned}
\delta & =\sum_{j, k, l} D_{j k l} \theta_{f p}^{i} y_{f p}^{i} \phi_{f p}^{i} \\
\theta_{t g} & =\sum_{j, k, l} T_{j k l} \theta_{f p}^{i} y_{f p}^{i} \phi_{f p}^{i} \\
y_{t g} & =\sum_{j, k, l} Y_{j k l} \theta_{f p}^{i} y_{f p}^{i} \phi_{f p}^{i} \\
\phi_{t g} & =\sum_{j, k, l} P_{j k l} \theta_{f p}^{i} y_{f p}^{i} \phi_{f p}^{i}
\end{aligned}
$$

where $D_{j k l}, T_{j k l}, Y_{j k l}, P_{j k l}$ are polynomials up to $5^{\text {th }}$ order:

$$
D_{j k l}=\sum_{i=0}^{m} C_{i j k l}^{D} x_{f p}^{i}
$$

The above process assumes that the vertical position of the vertex $x_{t g}=0$. The actual value of $x_{t g}$ is obtained from the BPM vector. This is treated as a small perturbation around 0 that introduces a linear correction on reconstructed $\delta_{t g}$ and $\theta_{t g}$ (see src/THaExtTarCor.C 
in Analyzer source code for details):

$$
\begin{aligned}
& \theta_{t g}^{\text {corrected }}=\theta_{t g}+0.61 * x_{t g} \\
& \delta_{t g}^{\text {corrected }}=\delta_{t g}+x_{t g} / 5.18
\end{aligned}
$$

The standard HRS optics matrix has been well-understood through previous experiments [32] [56] [57] [58].

In this analysis we used the existing $G_{p}^{M}$ LHRS optics matrix [33] except for the L17-PK, L17-HS, L17-SRC1, L17-SRC2 kinematics where the LHRS Q1 magnet saturated as the spectrometer momentum went above $3 \mathrm{GeV} / \mathrm{c}$ (see Sec.2.5). To account for this saturation effect, we took dedicated optics data with the hydrogen target and the multi-foil target at each kinematic settings to re-optimize the optics matrix. See the experiment wikipage 49 for a complete list of optics run information.

\section{Coordinate Systems and Pointing Study}

The HRS is not rigidly connected to the pivot. Small relative movements between the spectrometer outer shell and magnets/apertures can happen when rotating the spectrometer. As a result the HRS is not pointing to the nominal hall center. Three Linear Variable Differential Transformers (LVDT) installed inside the HRS shell are used to measure the horizontal offset(EPICS variable: HacL_CalcPoint for LHRS) from the spectrometer central ray to the defined hall center. And the actual HRS angle between the HRS central ray and the beam line (HacL_CalcAngle) is calculated from the set angle from encoder considering those offsets.See Fig. 3-1, Beside this horizontal miss-pointing. Both horizontal and vertical offsets can be obtained from a dedicated spectrometer survey. 
There is also an offset (along the beamline) between the target center and the ideal tar-

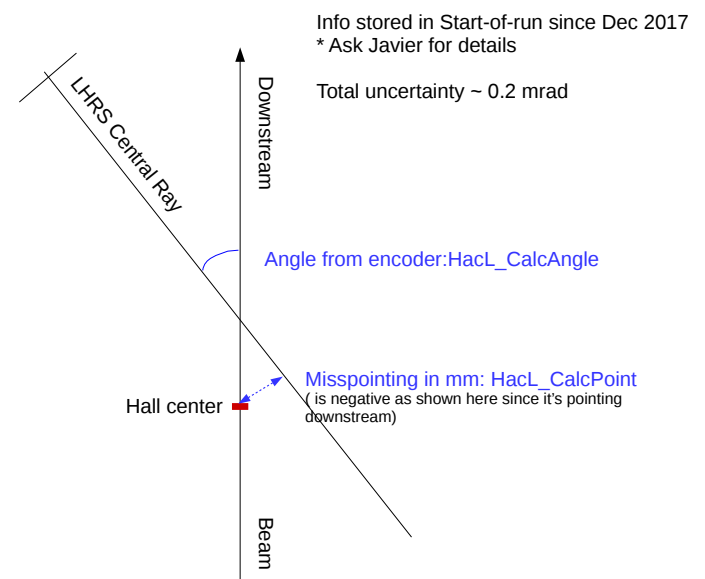

Figure 3-1: Schematics of LHRS misspointing information provided by the encoder.

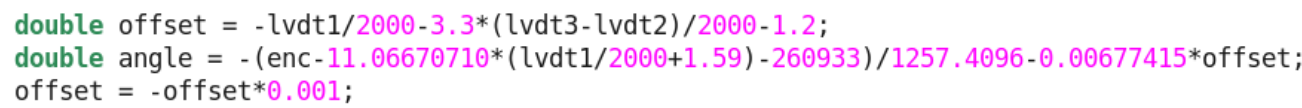

Figure 3-2: Code to calculate LHRS angle and offset from encoder and lvdt signals at each start of run on adaq computer (filename: tritium_db_L.C). The angle and offset are written into run database and used by Analyzer to reconstruct reaction point. Note that by convention the offset is positive when the spectrometer is pointing upstream. However during this experiment run period the variable HacL_CalcPoint is recorded in the opposite way.

get position (vertex $\mathrm{z}=0$ ). With a foil target at the actual target center, this offset is approximately (in horizontal plane):

$$
\text { vertex } z=\text { beam } \mathrm{x} / \tan (\Theta)-\left(\text { target } \mathrm{y}+\mathrm{HacL} \_ \text {CalcPoint }\right) / \sin (\Theta)
$$




\begin{tabular}{lcccc}
\hline Kinematics & Run Number & Angle & Calculated $\mathbf{z}(\mathbf{m m})$ & L.tr.vz mean $\mathbf{( m m})$ \\
\hline L17-PK & 3624 & 17.0088 & 6.77 & 6.81 \\
\hline L17-HS & 3495 & 17.0088 & 6.09 & 6.02 \\
\hline L17-SRC1 & 3682 & 17.0088 & 6.77 & 6.85 \\
\hline L17-SRC2 & 3684 & 17.0088 & 7.11 & 7.19 \\
\hline
\end{tabular}

Table 3.1: Carbon foil z position from L17 kinematics.

where $\Theta$ is the LHRS angle from encoder (HacL_CalcAngle). With LHRS we have variable L.tr.tg_y for target y. Lrb.x is used as beam x position when the raster is on. Otherwise Lurb.x is used.

A full reaction vertex reconstruction is handled by Analyzer. It is stored as L.tr.vz eventby-event in decoded rootfiles. Table 3.1 gives the mean value of vertex z distribution from carbon foil data calculated from equation 3.1 and from the analyzer output. On average the carbon foil is $6.7 \mathrm{~mm}$ downstream of the hall center. This number is used as the target offset in transverse position calibrations. Its standard deviation of $0.5 \mathrm{~mm}$ translates to a combination of transverse position uncertainty of $\sin (\Theta) \cdot 0.5 \mathrm{~mm}<0.1 \mathrm{~mm}$, and an in-plane angle uncertainty of $\sin (\Theta) \cdot 0.5 \mathrm{~mm} / 1.07 \mathrm{~m}<0.1 \mathrm{mrad}$, both of which are negligible.

\section{Transverse Position and Angle Calibration}

The $25 \mathrm{~cm}$ multifoil target data with sieve on provided sufficient information for both $y_{t g}$ and $\phi_{t g}, \theta_{t g}$ calibrations. Unrastered beam was used to improve resolution. With the information on target offset, beam position, and HRS misspointing, electron events from each foil were selected and optimized to its corresponding nominal position simultaneously. Fig. 3-5 shows the optimized vertex $z$ distribution of each foil with an up to $0.2 \mathrm{~mm}$ offset and $\sim 1.5 \mathrm{~mm}$ smearing ( only consider the production vertex $z$ range of $z_{t g} \pm 8 \mathrm{~cm}$ which is covered by foil 2 to foil 7 ). The entrance of the LHRS is $1.07 \mathrm{~m}$ away from the hall center, 


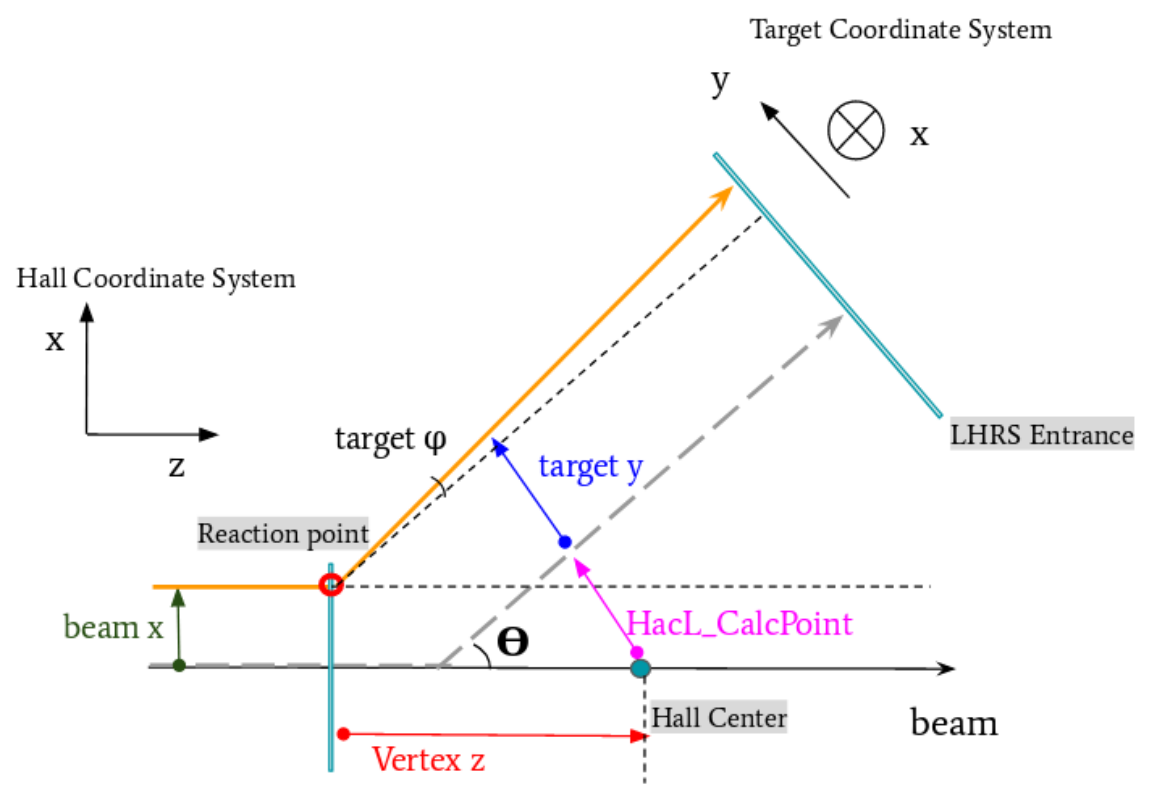

Figure 3-3: Schematic of LHRS pointing. Grey dashed line is the nominal central ray. orange solid line is the actual electron track. The setting as shown has a positive HRS offset since the central ray is pointing to the upstream of hall center, and the target position is negative.

so the $0.2 \mathrm{~mm}$ offset in $y_{t g}$ corresponds to a $0.2 \mathrm{mrad}$ offset on $\phi_{t g}$. Similarly the $1.5 \mathrm{~mm}$ smearing is a $1.5 \mathrm{mrad}$ uncertainty on $\phi_{t g}$.

To optimize $\theta_{t g}$ and $\phi_{t g}$ we installed a 1-inch-thick tungsten sieve slit at each HRS entrance (see Fig. 3-6). The reconstructed particle tracks from each foil are projected to the sieve position $\left(z_{t g}=+1.07 \mathrm{~m}\right)$ to form a sieve pattern as shown in Fig. 3-7. Then events from each sieve hole are selected and optimized to the nominal position. The HRS Angle determination accuracy of $0.6 \mathrm{mrad}$ in $\theta_{t g}$ and $0.2 \mathrm{mrad}$ in $\phi_{t g}$ [57, were combined with the $0.2 \mathrm{mrad}$ uncertainty from $y_{t g}$ to yield $\mathrm{a} \sim 0.3 \mathrm{mrad}$ total uncertainty on the central scattering angle. 


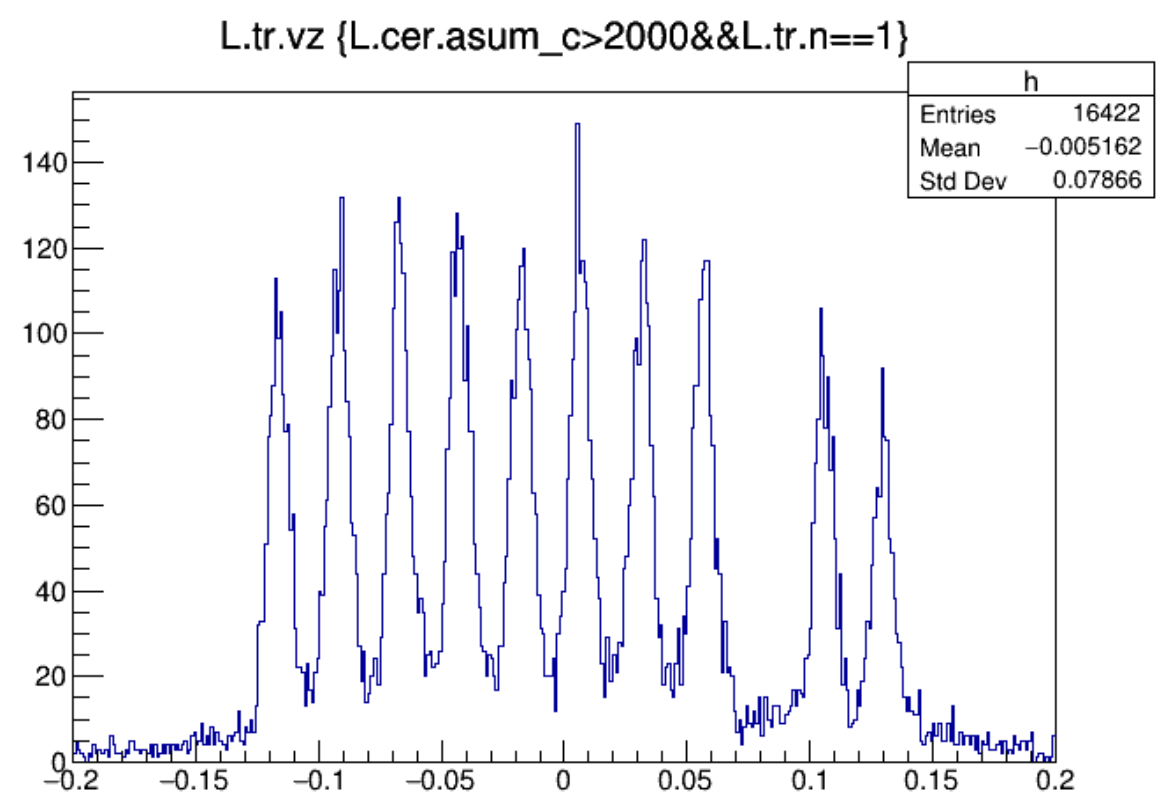

Figure 3-4: The reconstructed vertex z position of the multifoil target. The $25 \mathrm{~cm}$ long target consists 11 carbon foils evenly spaced. But in the target installation for the 2018 fall run period one foil was damaged. So we see only 10 peaks on this plot.

\section{Momentum Check}

With our kinematic settings the hydrogen elastic peak lies on a small corner of focal plane phase space, and the carbon excitation states have no statistics. Therefore a complete momentum calibration/scan is not possible. But with some hydrogen elastic data at 13.2 degree, we were able to tune the D100, D200 terms in $\delta_{t g}$ matrix manually to make the invariant mass uncorrelated with $\theta_{t g}$ (see Fig. 3-8). Fig. 3-9 shows the hydrogen invariant mass from L17-PK and L17-SRC1 after momentum calibrations and energy loss corrections. The hydrogen peaks locate at $938.4 \mathrm{MeV}$ and $939.4 \mathrm{MeV}$ instead of the ideal $938 \mathrm{MeV}$. This $\sim 1.5 \mathrm{MeV}$ offset is considered as the $\delta_{t g}$ accuracy. 

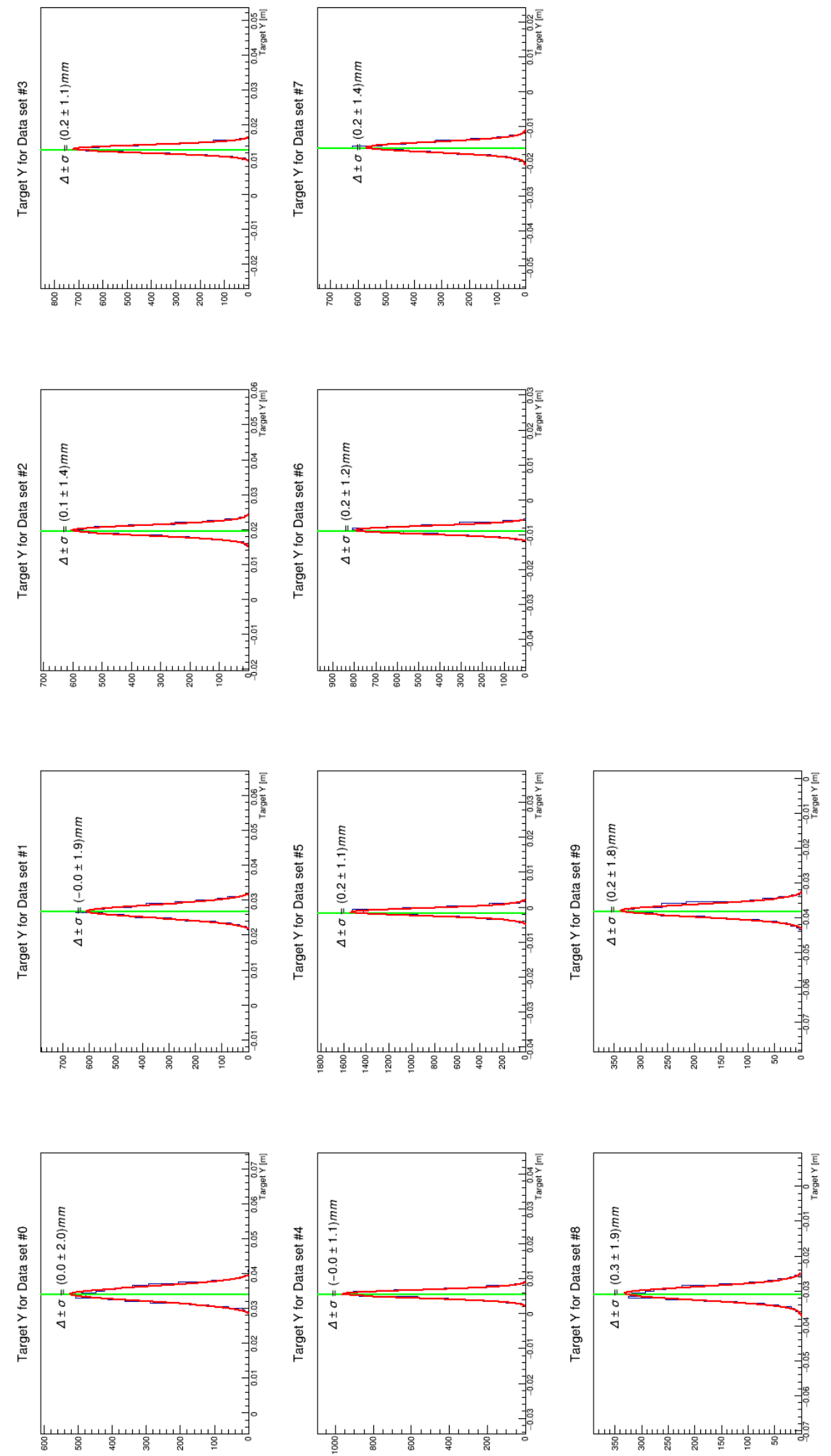

Figure 3-5: The optimized $y_{t g}$ of each foil (red) comparing to the nominal foil position (green) for L17-SRC2. 

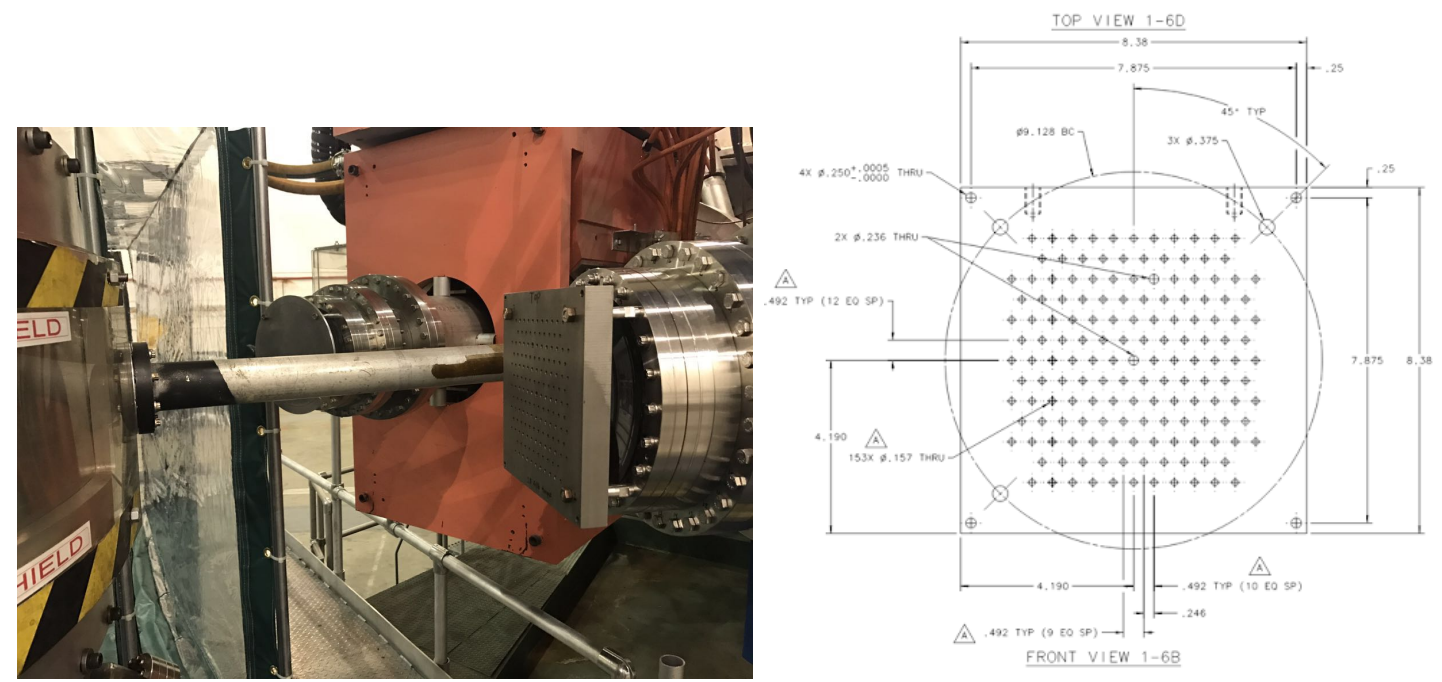

Figure 3-6: Left: The sieve is installed at the entrance of RHRS. Right: A drawing of sieve design.
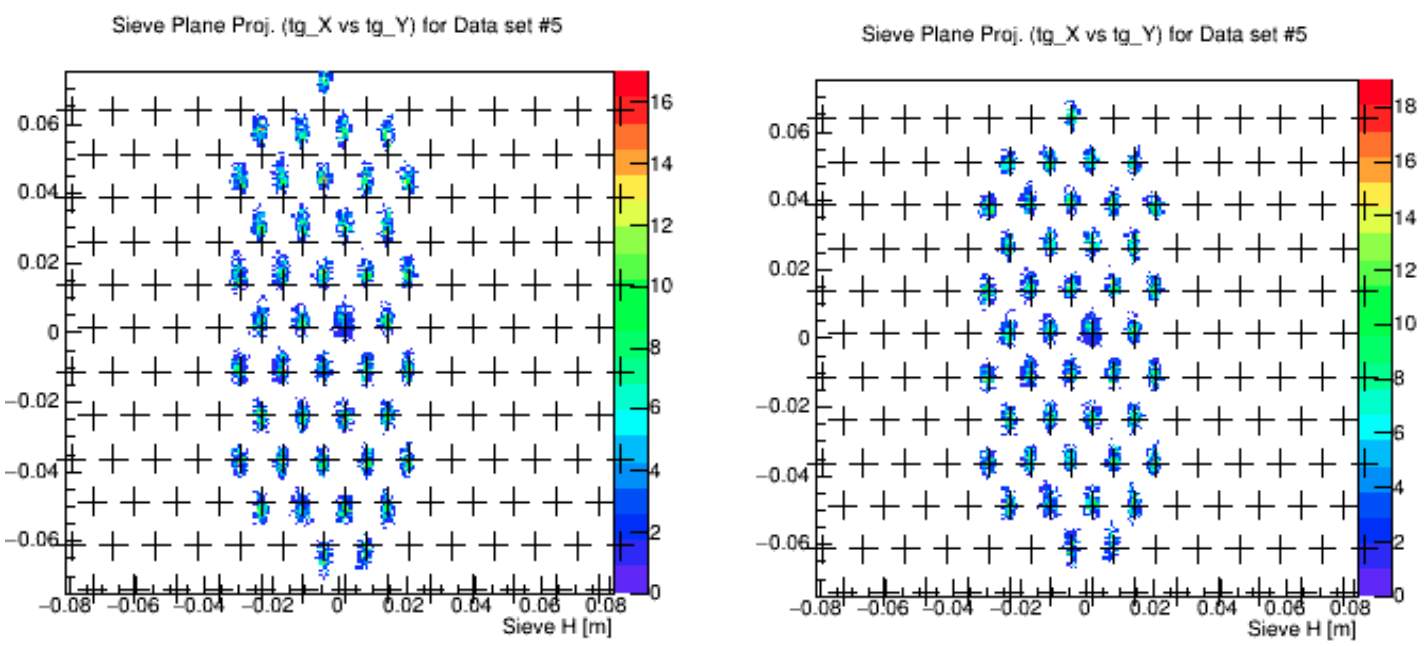

Figure 3-7: Sieve pattern of the central foil before (left) and after (right) $\phi_{t g}$ calibration for L17-SRC2 kinematics.

\subsubsection{Energy loss}

As the electron passing through materials it is slowed down by the nucleus field and radiates photons. This so-called bremsstrahlung radiation is responsible for most of the scattered electron energy loss at target at our kinematics, and is considered as a correction factor in our cross section extraction, see 4.3 .2 for details. 

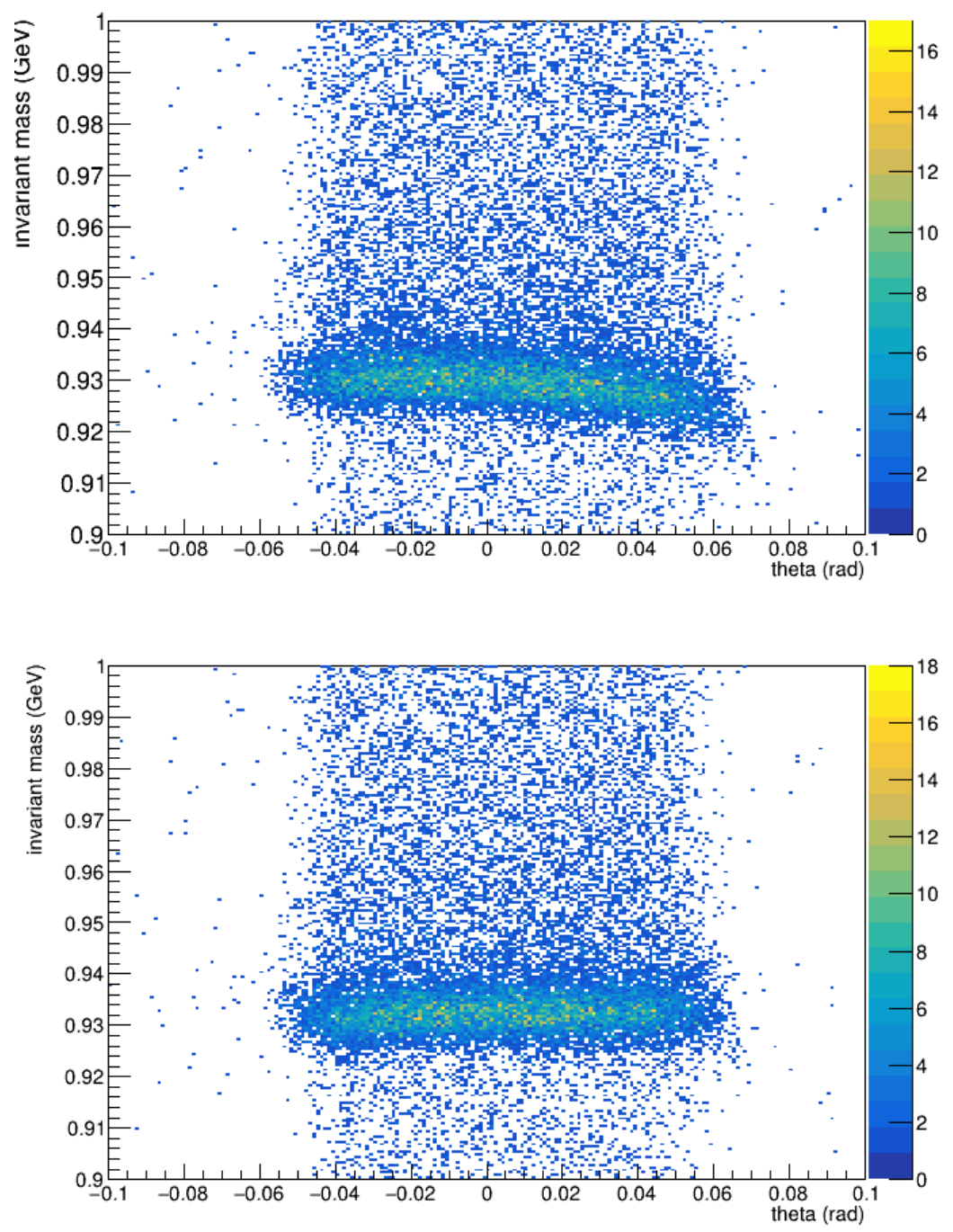

Figure 3-8: Hydrogen elastic calibration run 111702 at 13.2 degree during e'K experiment, momentum was set at $3.93 \mathrm{GeV}$ to match the L17-SRC2 kinematics. Plots show hydrogen invariant mass with respect to the out-of-plane angle $\theta_{t g}$ before (top) and after (bottom) manually tuning D100, D200 terms. After calibration the invariant mass becomes uncorrelated with $\theta_{t g}$.

On the other hand the scattered electron loses a few MeV energy due to ionization as it goes through the target wall, scattering chamber window, HRS entrance, etc, before it reaches the focal plane. And the incoming electron also lose an average of $0.3 \mathrm{MeV}$ energy on beam pipe window and scattering chamber windows before scattering. Materials along the electron path are shown in Fig. 3-10. The location, thickness and radiation length of each 

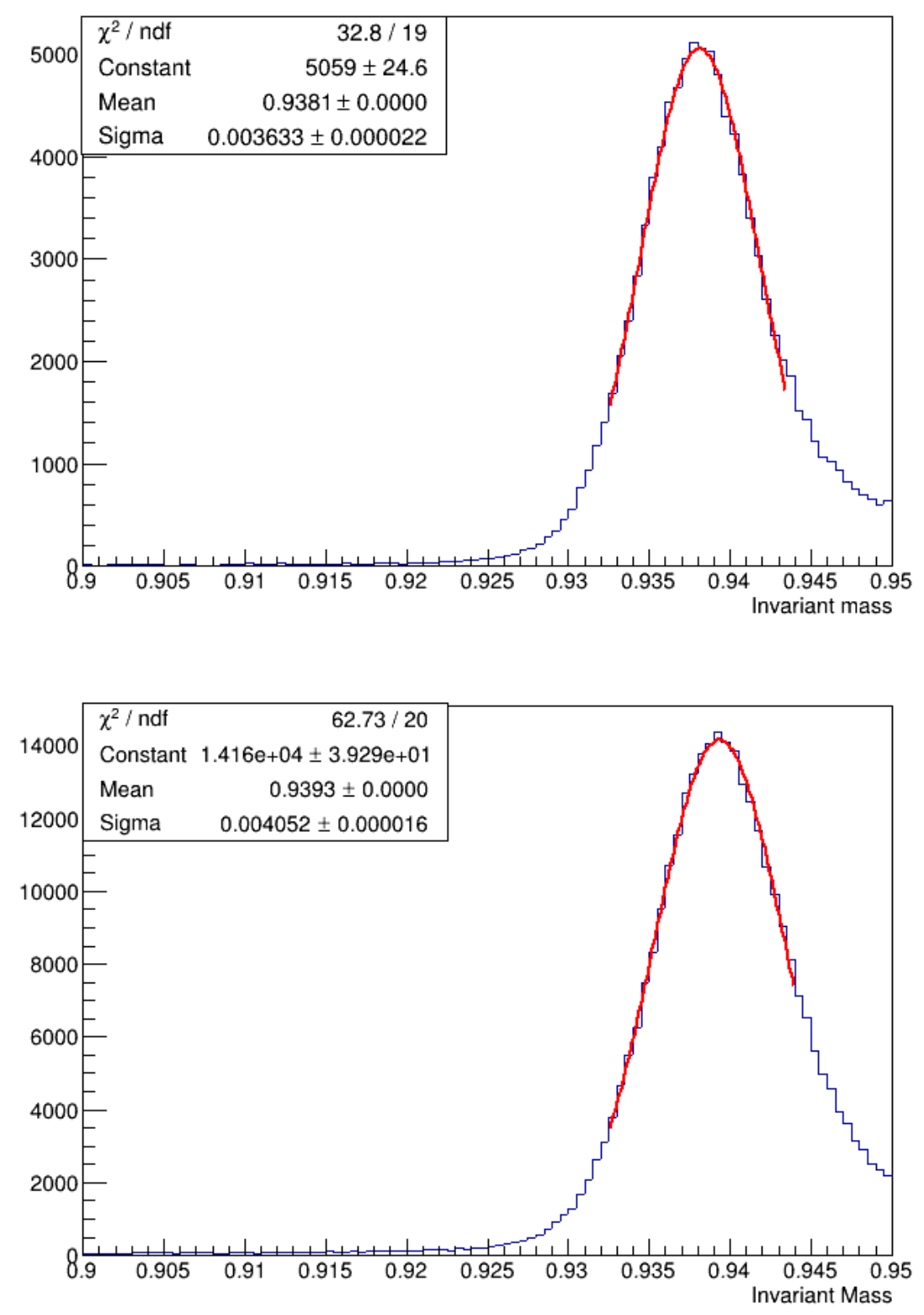

Figure 3-9: The hydrogen invariant mass from L17-PK (top) and L17-SRC1 (bottom) after calibration.

layer of material were provided to Analyzer to calculate the most probable energy loss (see Equation 1.22 in [59]). 


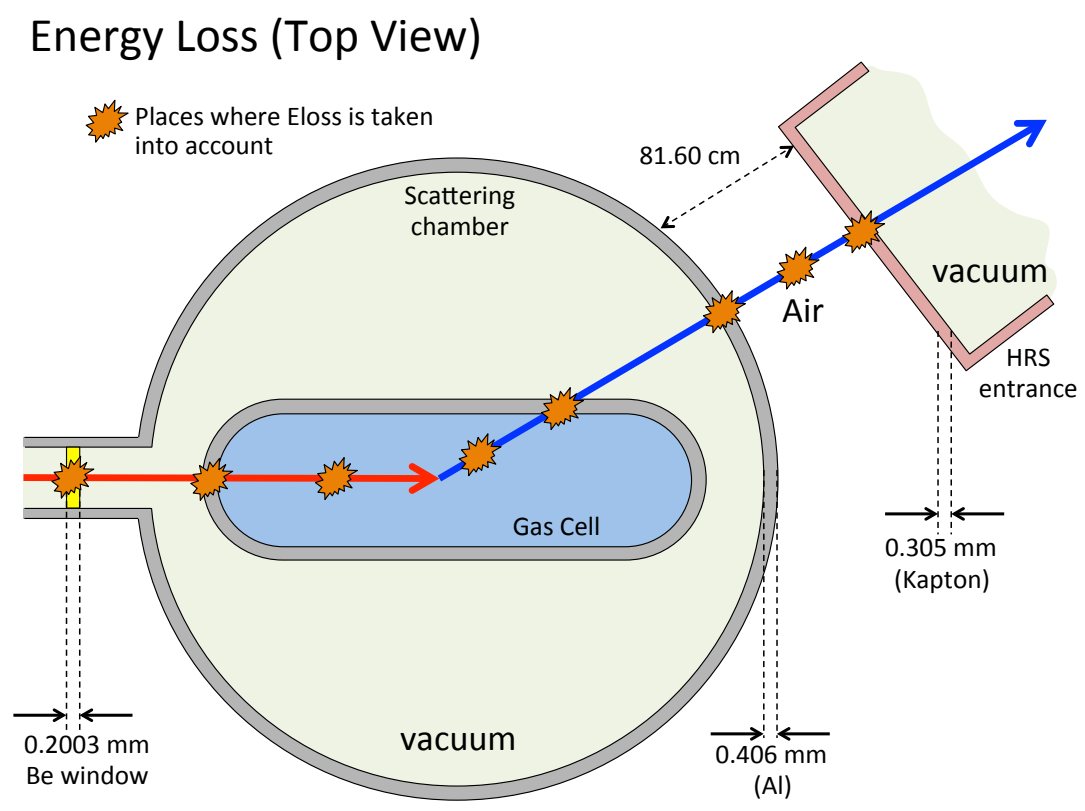

Figure 3-10: Schematic of incident and scattered electron energy loss 60.

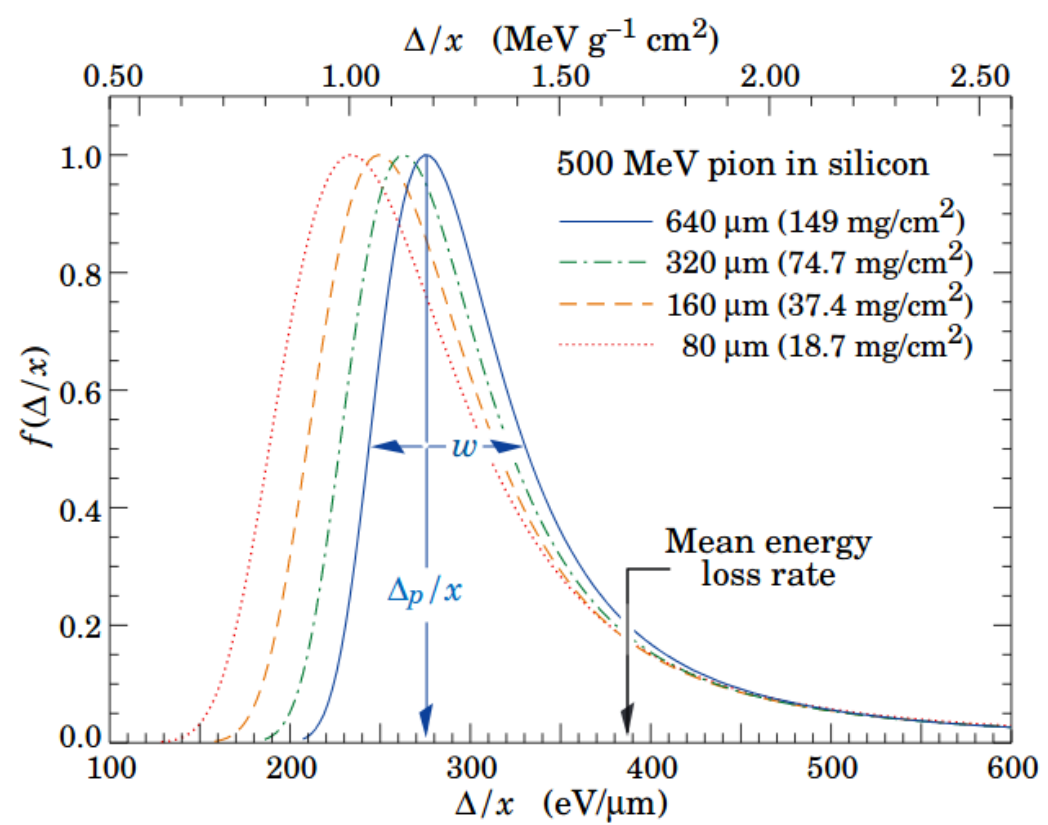

Figure 3-11: The energy loss rate of $500 \mathrm{MeV}$ pion goes through silicon. Figure taken from figure 32.8 in 61 to show that the most probable energy loss is a better approximation to the struggling function comparing with the mean energy loss. 


\subsection{Event Selection and Efficiencies}

We require a good electron event to have:

1. a stable beam current,

2. the production trigger (T2) fired,

3. a Chereknov sum signal greater than 1500 (more than 5 photonelectrons are collected),

4. the ratio of pion rejector sum to the particle momentum from tracking $(E / P)$ greater than 0.7 ,

5. only one track,

6. the reconstructed target variables passed the loose acceptance cuts of

- $-30 \mathrm{mrad}<\phi_{t g}<30 \mathrm{mrad}$,

- $-60 \mathrm{mrad}<\theta_{t g}<60 \mathrm{mrad}$,

$-4.0 \%<\delta_{t g}<4.5 \%$.

We need to know the the electron inefficiency, i.e. the probability of a good electron not passing these cuts, and understand any background contamination, i.e. events which pass the cuts but are not good electrons.

\subsubsection{Beam Quality Check}

To eliminate uncertainties from the gas target density fluctuation, we only included data collected with the requested $22.5 \mu \mathrm{A}$ stable beam in this analysis (which means the remain of this thesis). We start from identifying the actual mean beam current of a run from the 
scaler BCM counts (see Sec. 2.3.2), then select events that were collected 5 seconds after the beam was stable within $\pm 1.5 \mu \mathrm{A}$ of the mean current. Fig. $3-12$ shows how to find events with stable beam and calculate the associated beam charge and number of production T2 triggers with scaler output. The livetime of the DAQ system (the portion of time that

The scaler information was inserted into data stream frequently. All counts are cleared at the start of run. Counts between the $\mathrm{i}^{\text {th }}$ and $\mathrm{i}+1^{\text {th }}$ updates were used to get:

\section{$I_{i} \quad$ : beam current;}

$d N_{i}$ : number of triggers sent to trigger supervisor; $d t_{i}$ : time interval from a $103,700 \mathrm{~Hz}$ clock counts.

The deposited charge $\mathrm{dQ}_{\mathrm{i}}=I_{i} d t_{i} . \mathrm{NS}_{\mathrm{i}}$ is the number of triggered events in this time interval.

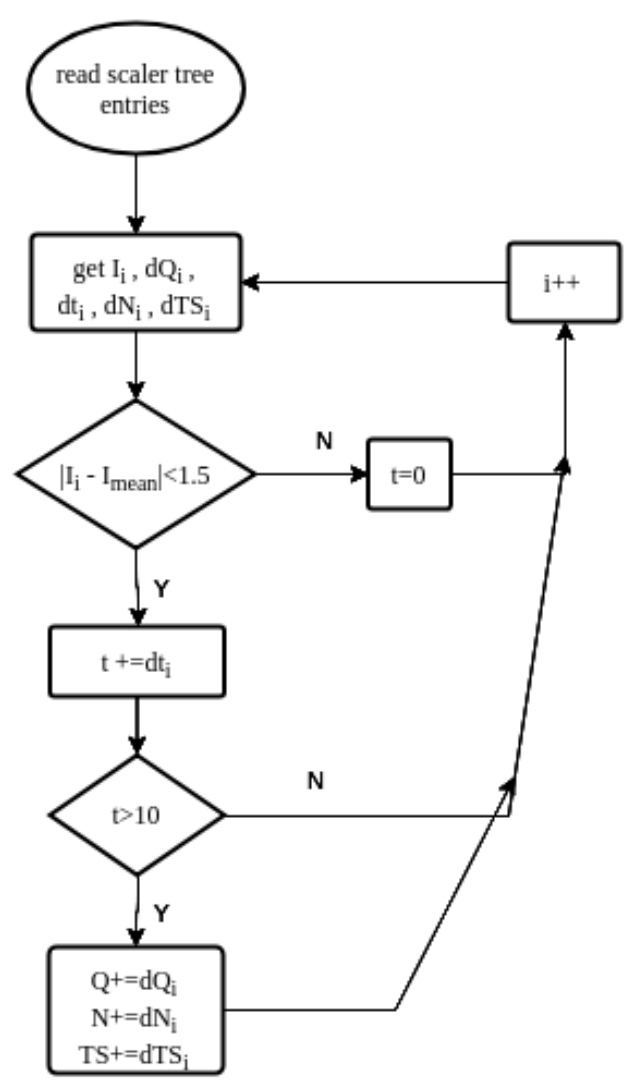

Figure 3-12: Flow chart on selecting events with stable beam.

DAQ is able to take trigger events) is given by:

$$
\text { livetime }=\frac{\# \text { of events with T2 trigger }}{\# \text { of T2 trigger signals recorded by scaler }}
$$



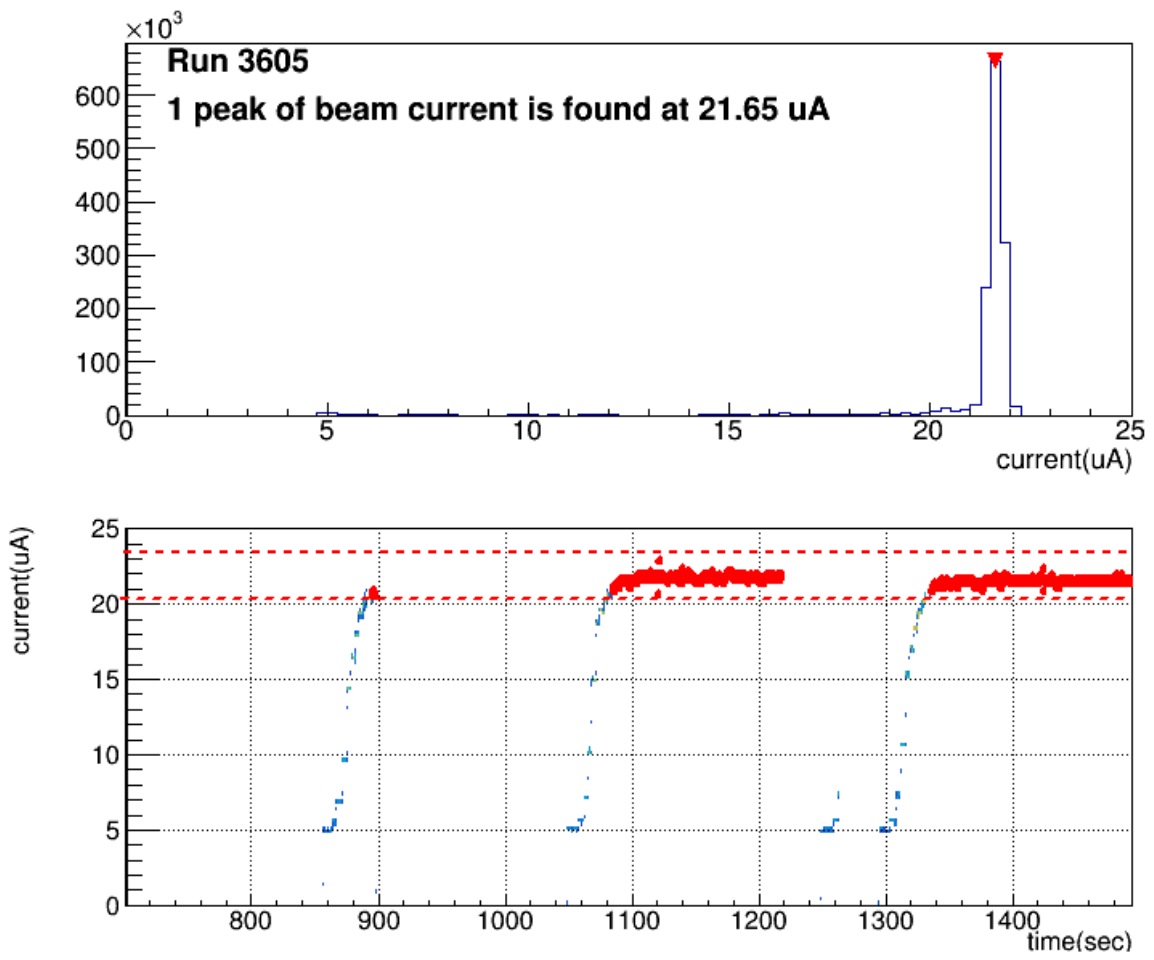

Figure 3-13: Example of event selection with run 3605. Top: the 1-d spectrum of beam current. Bottom: a zoomed-in plot of beam current versus time to show how the beamtrips were excluded. Events that fall into $\pm 1.5 \mu \mathrm{A}$ of mean current (between two dashed line, with red markers) are the events that were used in analysis.

\subsubsection{Trigger Efficiency}

A good electron event collected by the HRS are expected to have large Cherenkov and pion rejectors singals, particle velocity close to speed of light, only one reconstructed track within our loose acceptance cuts. Such an event would fire all three triggers. But the electron may fail to fire the production trigger T2 ((S0 \& S2) \& Cer) if the Cherenkov or Scintillators were not responding or their generated signals didn't form a trigger. For example, if there was a failure on one scintillator, the T3 trigger ((S0 \| S2) \& Cer) would have fired but not T2 or T1 (S0 \& S2). Similarly, the Cherenkov deficiency produced events with only T1 fired. 
Fig. 3-14 shows the percentage of events with different trigger combinations. Less than $1 \%$ of good electron events failed to fire all three triggers at the same time. Most of those events fired T3, but failed to fire T1 and T2 due to missing S0 signals. We projected their tracks from VDC plane to the Q3 exit aperture and the S0 plane to study their spatial distributions. As shown in Fig. 3-15, their tracks are concentrated at the position of S0 lower frame (in the detector coordinate system vertical axis $x$ is pointing down). Therefore we concluded that T3-only events are mainly out-of-acceptance electrons that rescattered on the S0 frame. They should be rejected by the good electron event selection. There is no need to compensate this loss with the trigger efficiency.

The combined trigger efficiency (to account for T1-only events) was evaluated run-by-run. The result is above $99.5 \%$ for all kinematic settings. The counting uncertainty in this calculation is negligible.

\subsubsection{Particle Identification}

Fig. 3-16) shows the distribution of Cherekov sum signal versus the ratio of pion rejector sum signal to the momentum of reconstructed $\operatorname{track}(E / P)$. With our HRS momentum setting a scattered electron should trigger the Cherenkov detector and deposit large amount of energy in pion rejectors, which corresponding to the large bright cluster at $E / P=1$. Pion is the primary source of contamination, which creates very small pion rejector signal and can not trigger Cherenkov. Pion may knock out electrons through ionization at or before Cherenkov. This so-called delta electron has very low momentum but enough to trigger Cherenkov, and creates events with large Cherenkov signal but very small pion rejector signals. The pion can also produce photons through charge exchange $\left(\pi^{-} n \rightarrow \pi^{0} p \rightarrow \gamma \gamma\right)$. 
Cuts: track $==1$, cer $>1500, E / P>0.7$, abs(th,ph,delta) $<60$ mrad, 40 mrad, $5 \%$

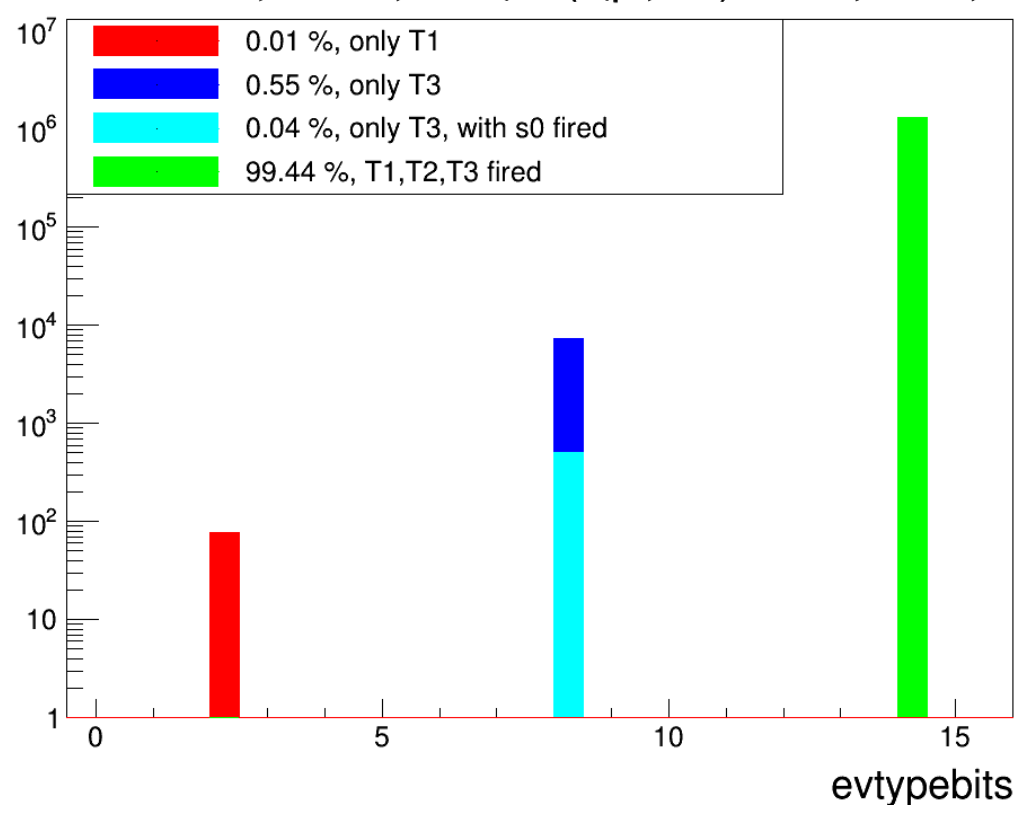

Figure 3-14: Trigger type distribution from run 100684. The $\mathrm{x}$ axis is the binary sum of trigger type. For example, an event with only T3 fired would have evtypebits $=2^{3}=8$. More than $99 \%$ of electron events fired all three triggers.
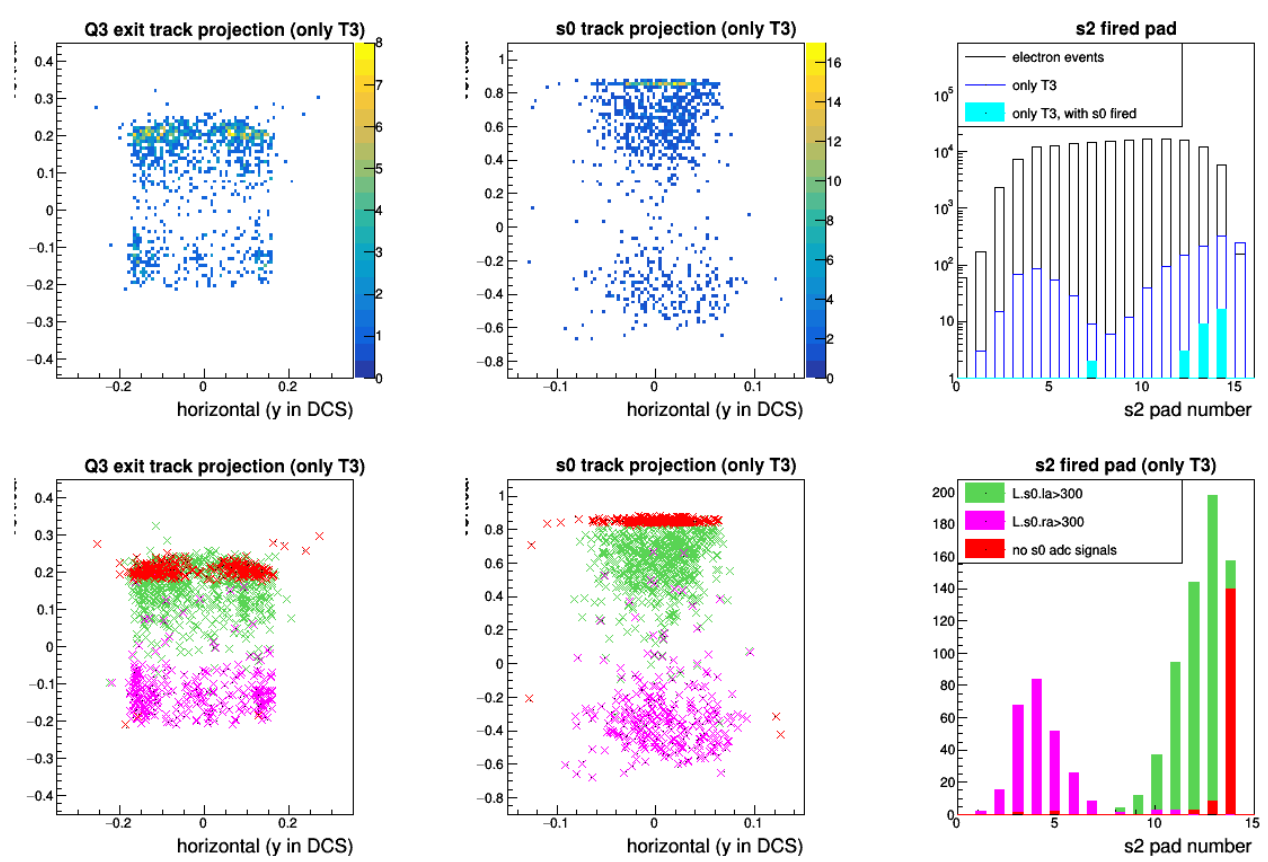

Figure 3-15: Distributions of T3-only events on Q3 exit aperture and the S0 plane, and the corresponding S2 signals. 
Those photons deposit all its energy on pion rejectors, and create an event with a small Cherenkov signal but large pion rejector signals.

A good electron event may also have small Cherenkov or pion rejector signals due to the

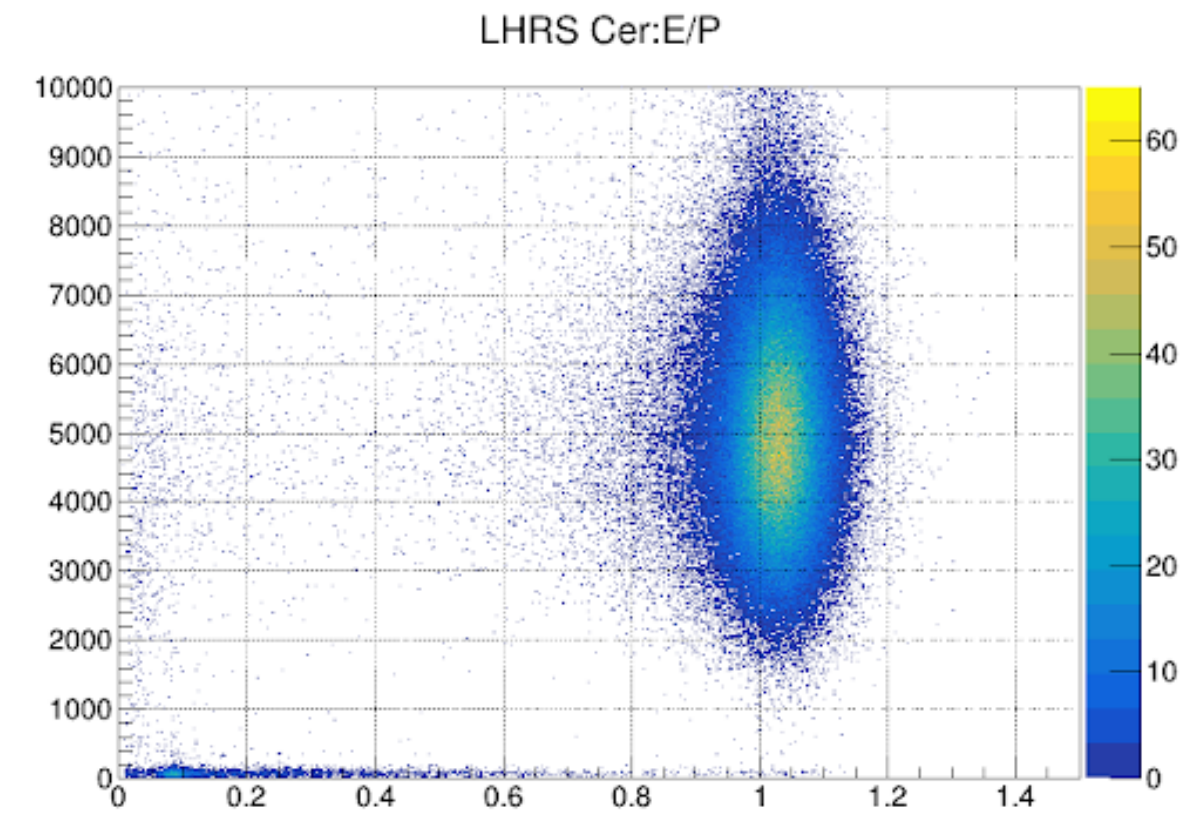

Figure 3-16: The Cherenkov signal versus E/P ratio after calibration.

detector inefficiency. We applied cuts on Cherenkov and pion rejector signals to select good electron events. The lost events in this process, which are the sum of pion contaminations and detector inefficiency are less than $0.5 \%$ of the total.

The sum of ten Cherenkov PMT singals after calibration is proportional to the total number of produced photons and can be described by Landau distribution. As shown in Fig. 3-17, on average a good electron induced 15 photons. So if we select events with Cherenkov sum signal greater than 1500 , the probability of missing a good electron event is negligible (about $0.01 \%$ ) according to the fitting. Also, by comparing the distribution before and after the pion rejector cut, we conclude that there is less than $0.01 \%$ of probability for a pion to 
pass the 1500 Cherenkov signal cut.

A good electron event should create large signals on both layers of pion rejectors. And the

L Cerenkov Sum

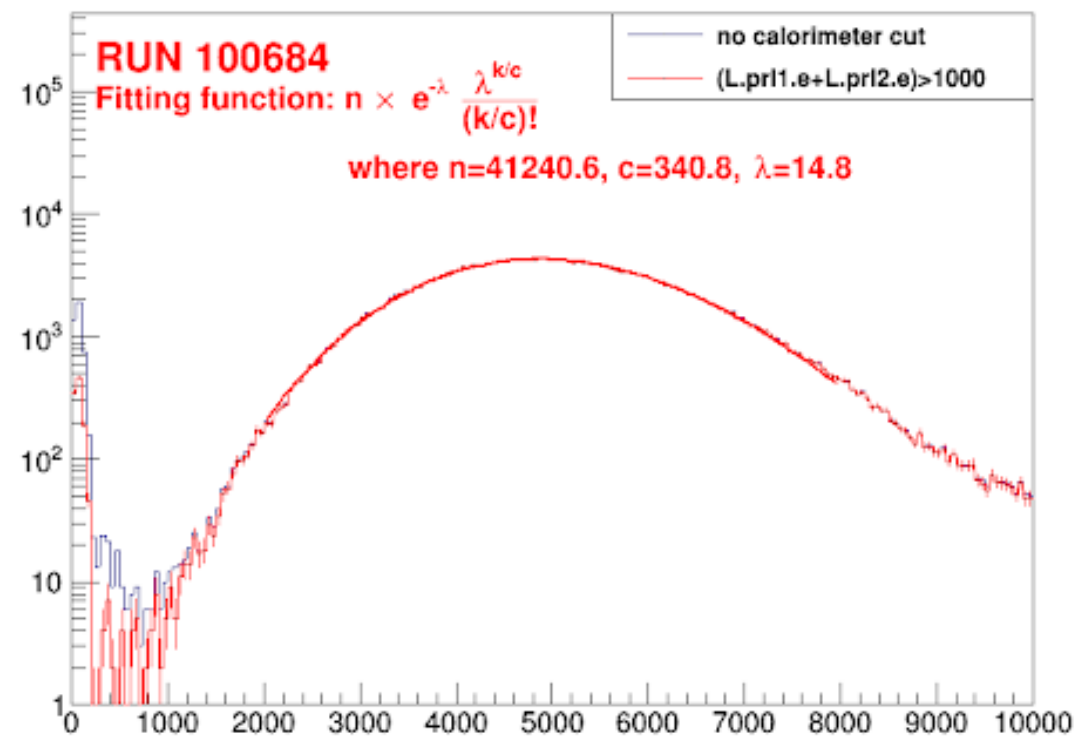

Figure 3-17: The peak of Cherenkov sum signal was fitted to a Poisson distribution.

sum of calibrated signals is proportional to the electron momentum. As shown in Fig. 318, the good electron events have a Gaussian-like distribution around $E / P=1$. We used a linear extrapolation of the non-Gaussian tail of this electron distribution to estimate that the probability of an electron with $E / P<0.7$ from this fitting is less than $0.5 \%$. Similarly, taking a Gaussian fit of the pion peak indicates that there is zero probability of a pion event having $E / P>0.7$.

The above process was repeated with various acceptance cuts. Results varies at the level of $0.1 \%$. Very often the time-of-flight(hence the velocity $\beta$ ) cut is applied to distinguish electrons and other particles, also to remove cosmic rays contamination. In our case the 

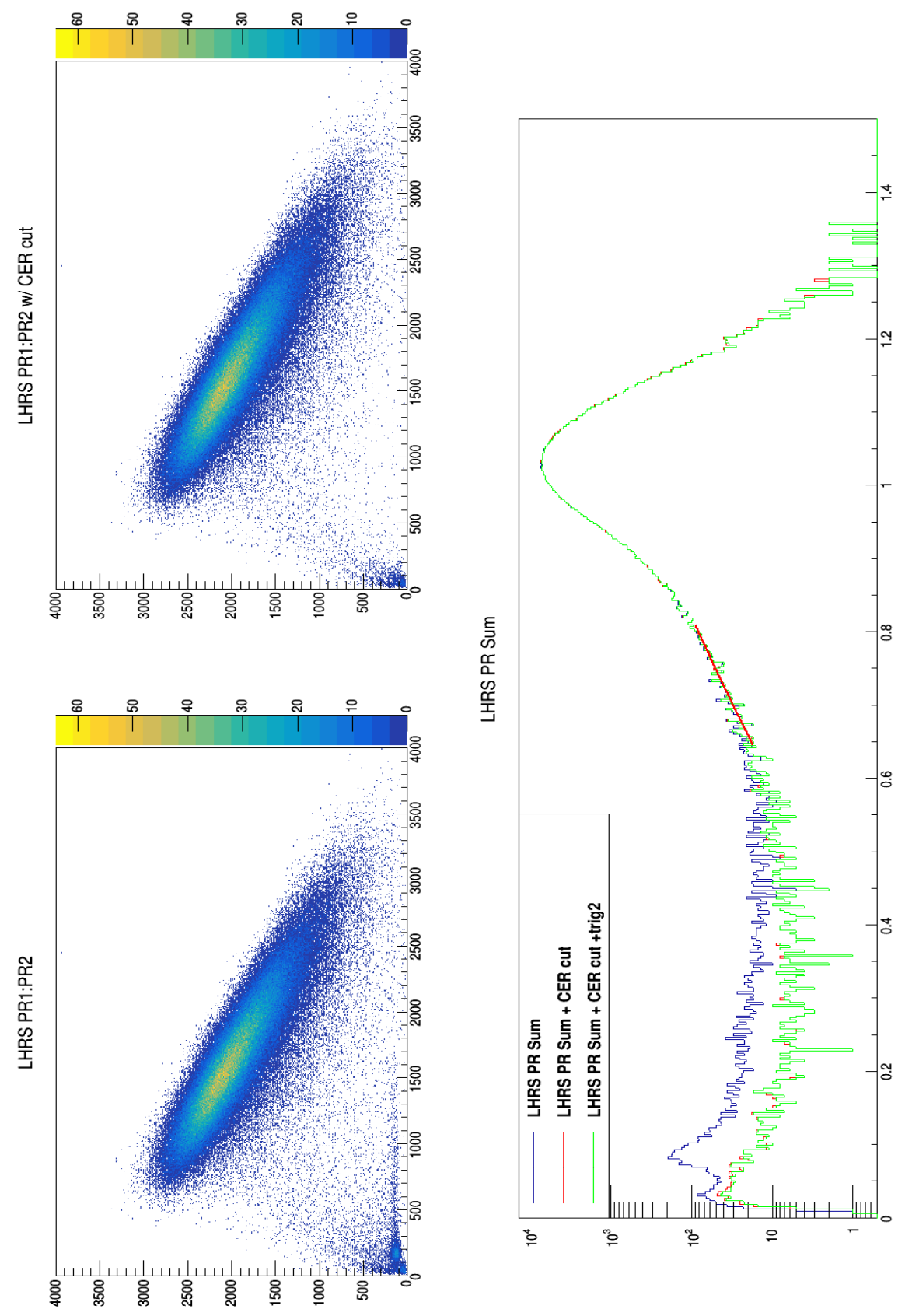

Figure 3-18: Upper: the first versus second layer of pion rejector signals before (left) and after (right) Cherenkov cut. Lower: the ratio of pion rejector sum signal to the track momentum. The red line indicates a linear extrapolation of the electron distribution to $E / P<0.7$. The blue peak at $E / P \nmid 0.1$ are mostly pions 
combination of Cherenkov $>1500$ and $E / P>0.7$ cuts already give $>99.5 \%$ PID efficiency. Pion contamination and events from cosmic rays are sufficiently removed by those PID cuts. Additional timing cut makes negligible difference therefore not applied.

\subsubsection{Tracking Efficiency}

As shown in Fig. 3-19, among events that passed PID cuts, $\sim 1 \%$ of them have no track mainly because no cluster is available in one of the four VDC planes. Those events are very likely mis-reconstructed good events and should be compensated by the the tracking efficiency. Also, there are up to $1 \%$ of events that have more than one reconstructed track (see Fig. 3-20). This number decreases to $0.4 \%$ if we apply the nominal acceptance cuts as well as a vertex $\mathrm{z}$ cut of abs(L.tr.vz $[0])<0.085 \mathrm{~m}$.

Fig. 3-21 shows that most of

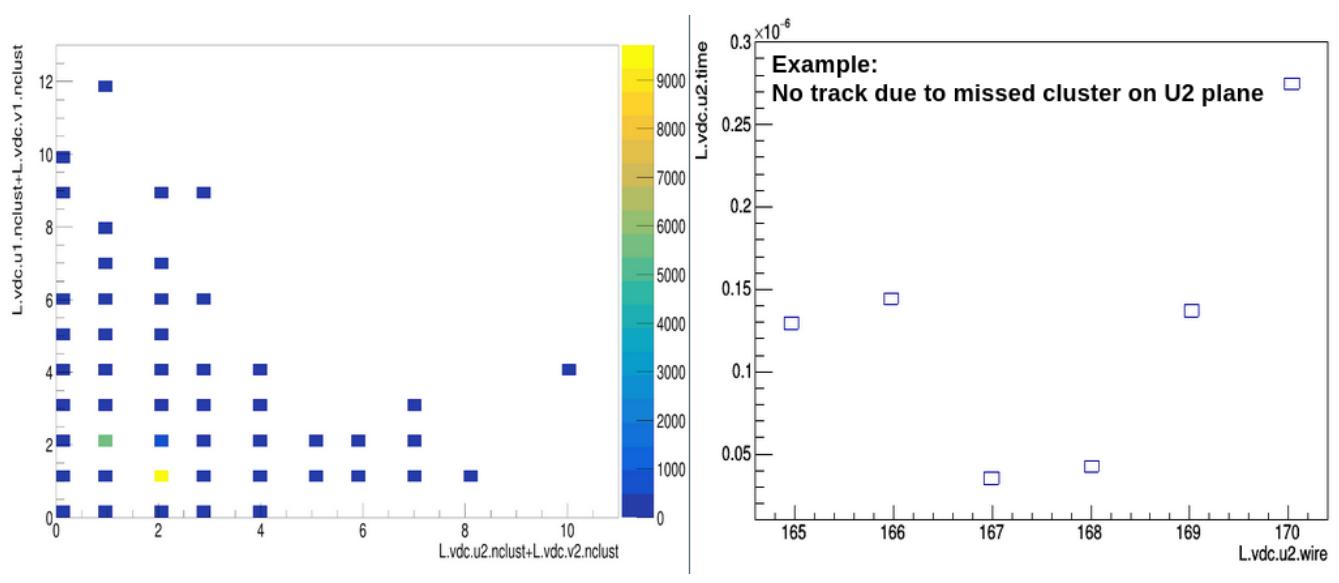

Figure 3-19: Left: distribution of no-track events with respect to cluster numbers. Right: TDC signals from U2 plane of a no-track event with three clusters. The algorithm failed to identify the "V" shape due to the unexpected rise on the left.

multi-track events locate outside the loose acceptance cuts. Very likely they were created by originally out-of-acceptance electrons that re-scattered on some apertures to reach the focal plane. They should not be counted as good electron events. The statistical uncertainty 


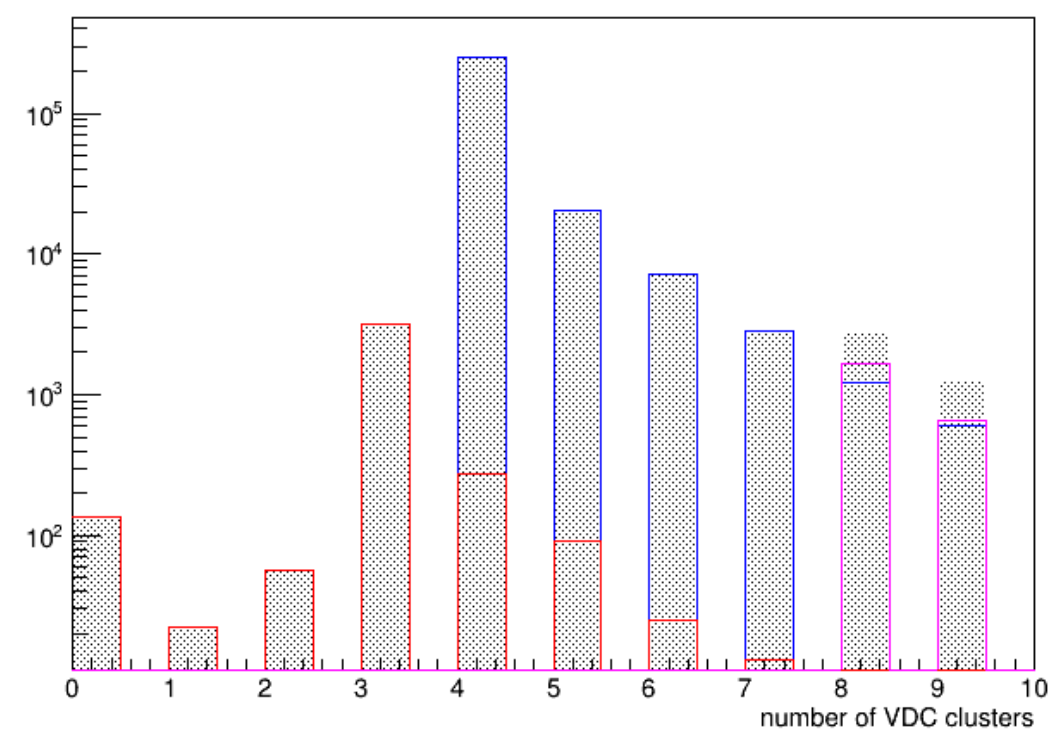

Figure 3-20: Total number of clusters from four VDC planes. Shaded area represents all events after PID cuts. No track events in red, one track events in blue, and multi-track events in purple.
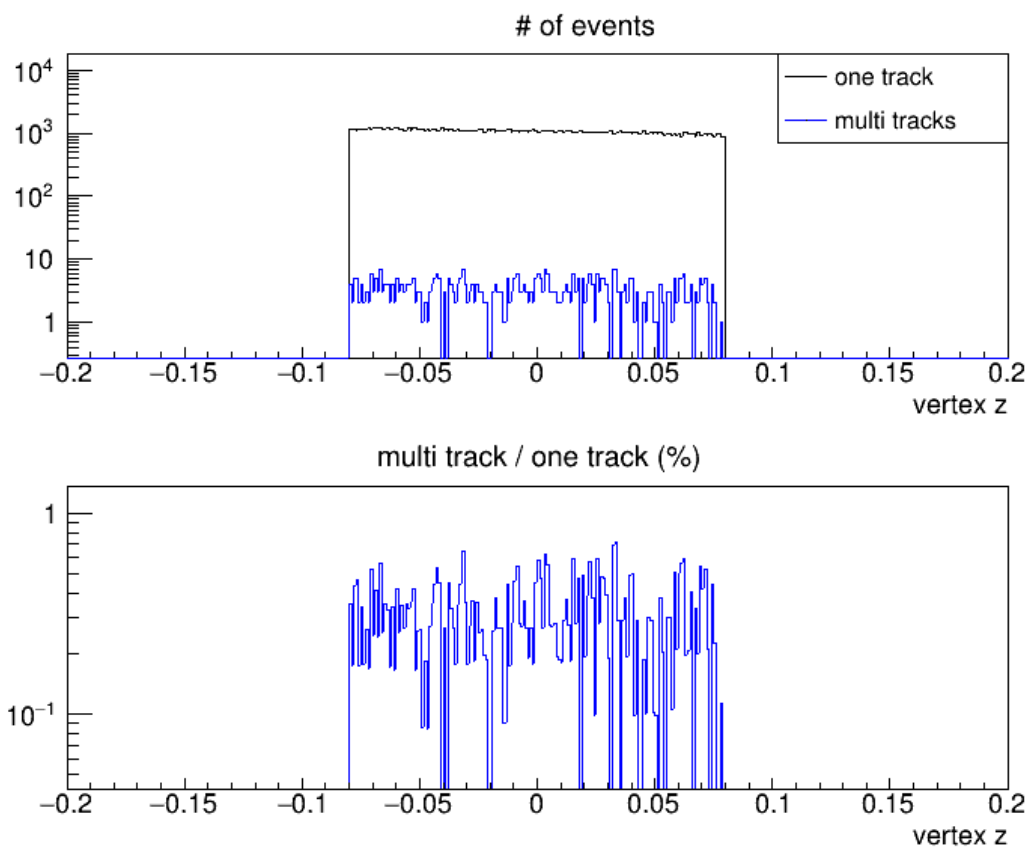

Figure 3-21: The amount of multi-track events with loose acceptance cuts and vertex $\mathrm{z}$ cut is less than $0.4 \%$ of all good electron events. 

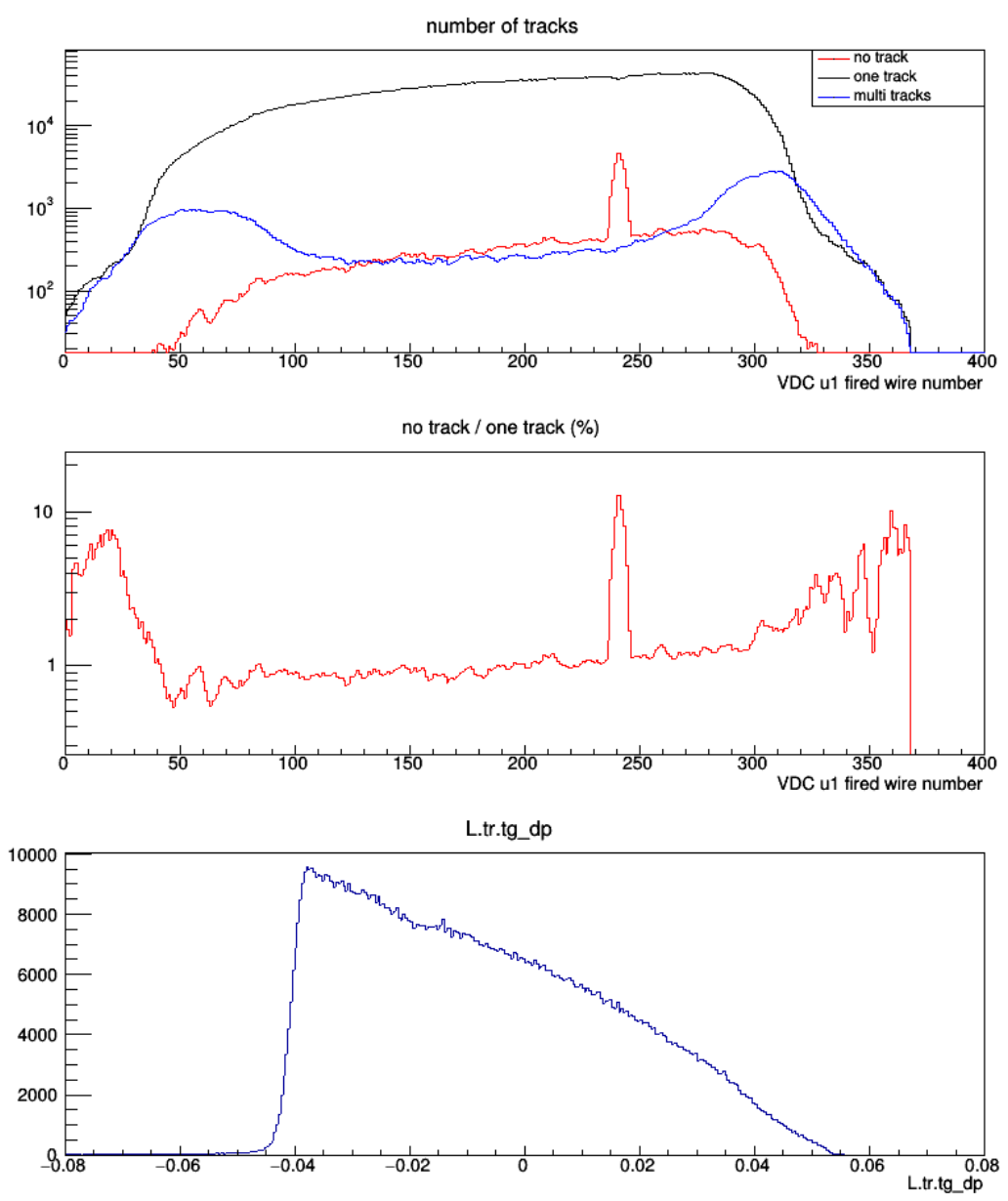

Figure 3-22: The percentage of no-track events versus the U1 plane. In the Spring 2018 run period the wire \#240 on U1 plane was noisy, which caused an inefficiency spike. This wire was fixed before the fall 2018 run period. This effect was demonstrated to be cancelled in the ratio analysis.

of tracking efficiency is negligible. A $0.2 \%$ correlated systematic uncertainty is assigned to account for the treatment to multi-track events.

During the Spring 2018 run period, a loose ribbon cable caused noise in the TDC signal from wire \#240 on U1 plane. Since the TDC is set to record the last 6 signals per trigger, such a noise somethings washed out the real hit information so that the Analyzer was not able to build a cluster, hence a track from that event. This wire was fixed before the fall 2018 run period. As a result, all the spring data, that is, all kinematics on Table 2.1 except L17 have 

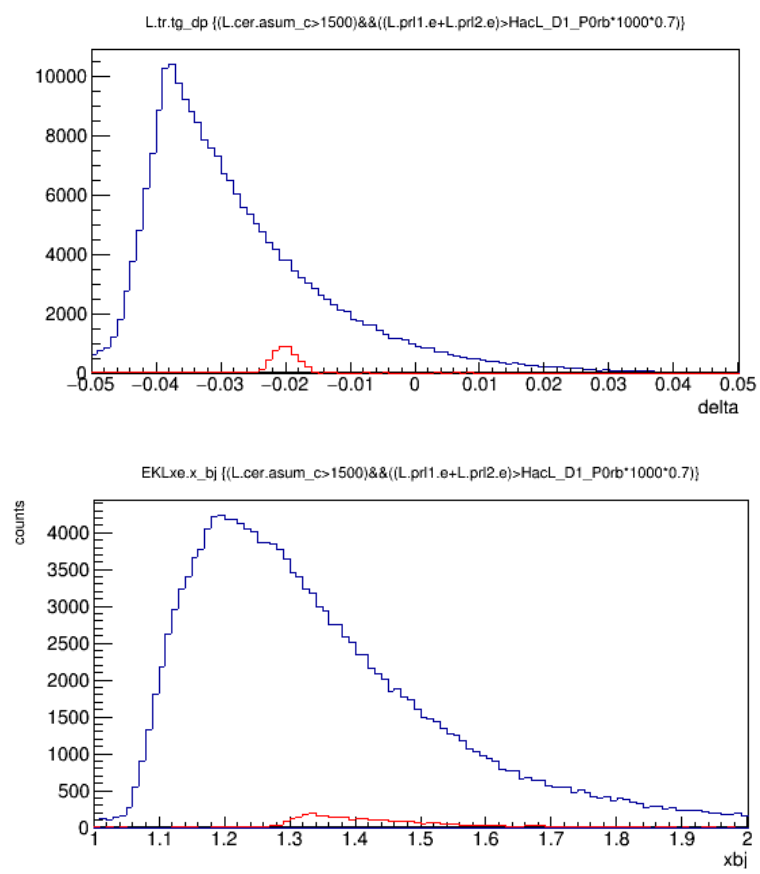

Figure 3-23: All good electron events (blue) and good electron events with U1 wire \#240 fired (red) are plotted with respect to momentum (top) and $x_{b j}$ (bottom).

a large local VDC inefficiency, which translates to a dip on $\delta_{t g}$ distribution at about -0.02 (see Fig. 3-22). This noisy wire contributes to $\sim 10 \%$ of total no-track events, or $0.1 \%$ of overall tracking inefficiency. This effect is cancelled in cross section ratios. To estimate the impact on absolute cross section extraction, we took a "good" run from L17-SRC2 to check the distribution of wire \#240-fired events. As shown in Fig. 3-23, $2 \%$ of electron events were reconstructed with a U1 cluster involving wire \#240. Those events have up to $5 \%$ local contribution to yield when binning in $x_{b j} .10 \%$ inefficiency on top of this gives up to $5 \% \times 10 \%=0.5 \%$ local inefficiency on a particular $x_{b j}$ bin. The analysis was repeated with other L17 kinematics, results are similar. 


\section{Chapter 4}

\section{Cross Section Extraction}

The inclusive QE cross section can be extracted from experimental dat as:

$$
\frac{d^{2} \sigma}{d \Omega d E^{\prime}}=\frac{\# \text { of events }}{Q \cdot \rho_{l} / M_{A} \cdot \text { efficiencies }} \frac{A\left(\Omega, E^{\prime}\right)}{\Delta \Omega \Delta E^{\prime}},
$$

where $A\left(\Omega, E^{\prime}\right)$ is the acceptance function that represents the probability of a particle with the scattering angle $\Omega$ and momentum $E^{\prime}$ to reach the HRS focal plane. The first part of the equation is called the "yield" of the data. The calculated yield needs to be corrected for contamination and other effects. Then it is compared to the Monte-Carlo simulation to study the acceptance effect, that is, $A\left(\Omega, E^{\prime}\right) /\left(\Delta \Omega \Delta E^{\prime}\right)$ in the above equation. Finally, the cross section can be extracted from the data to simulation comparison.

\subsection{Yield Calculation}

For a given production run $i$, events with stable beam current were first identified. Then we calculated the following quantities:

- $Q_{i} \quad$ : charge with stable beam current (see Sec. 3.3.1.

- $L T_{i} \quad$ : the livetime of the production trigger,

- $C_{i} \quad:$ good electron event counts per $x_{b j}$ bin, 
- $P S_{i} \quad$ : the prescale factor for the production trigger, which is set to 1 for this experiment,

- ef $f_{i} \quad:$ the product of all efficiencies including trigger, tracking, PID cut efficiencies,

- $\rho_{l} \quad$ : effective area density of the target without beam. For a gas cell it should represent the amount of gas after vertex z cut (linearly scaled by the target length),

- denscor ${ }_{i} \quad$ : the ratio of the effective gas target density at given beam current comparing to its density without beam. See Sec. 4.2 .2 for details.

The yield for this run is

$$
Y_{i}=\frac{\# \text { of observed events }}{\text { Effective Luminosity }}=\frac{C_{i}}{Q_{i} \cdot \rho_{l} / M_{A} \cdot \text { denscor }_{i} \cdot \text { eff } f_{i} \cdot L T_{i} / P S_{i}}
$$

with $\frac{1}{\sqrt{C_{i}}}$ as the fractional statistical uncertainty. $M_{A}$ is the nucleus molar mass.

Plotting the calculated yield along with efficiencies from runs with the same target and kinematics is a good way to check run qualities. For example, as shown in Fig. 4-1, all helium-3 runs at L17-SRC2 have consistent yield and efficiencies except one outstanding run 3865. Looking back in the logbook, there was a comment saying "Bad beam quality, stopped early". Therefore we removed that run from our production run list.

The overall yield of a given kinematics is the weighted arithmetic mean of all good production runs under this kinematics:

$$
Y_{\text {overall }}=\frac{\sum_{i} C_{i}}{\sum_{i} Q_{i} \cdot \rho_{l} / M_{A} \cdot \text { denscor }_{i} \cdot e f f_{i} \cdot L T_{i} / P S_{i}}
$$

with a fractional statistical uncertainty of $\frac{1}{\sqrt{\sum_{i} C_{i}}}$. The systematic uncertainties are listed 


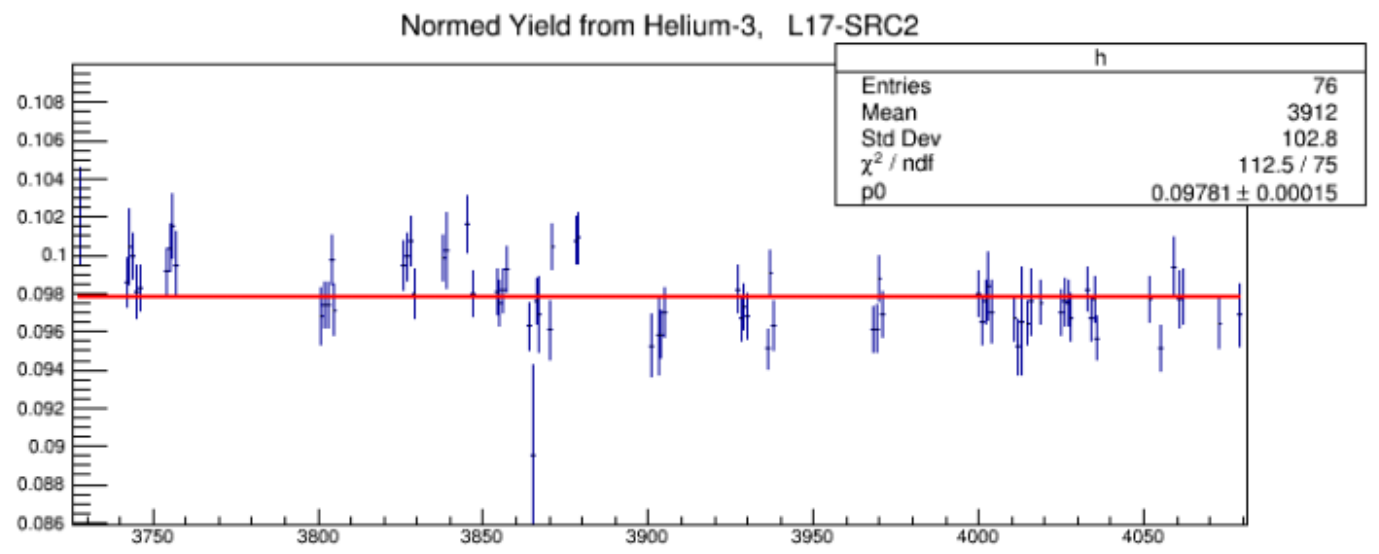

Efficiencies

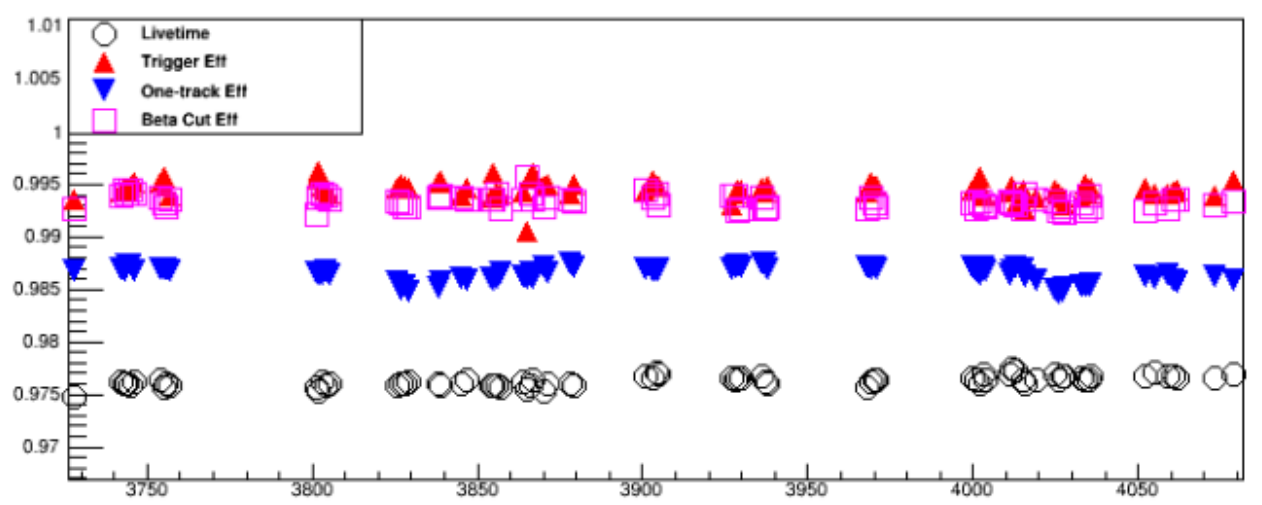

current

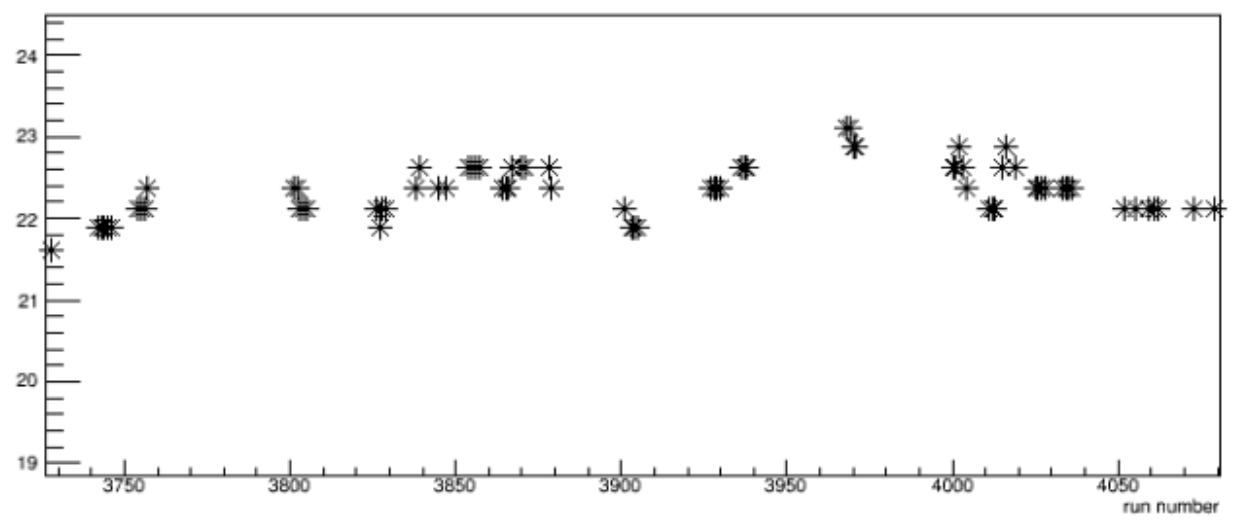

Figure 4-1: Normalized yield, efficiencies, livetime, and beam current for every L17-SRC2 run with Helium-3 target.

in Table 4.1 


\subsection{Gas Target Corrections}

\subsubsection{Endcap Contamination}

Electrons scattered off the entrance and exit window of a gas cell might be mis-reconstructed into the gas body due to the resolution smearing and multiple scattering. Since the endcap's thickness is comparable to the gas target body, this effect could be a significant source of contamination depends on the kinematics.

As shown in Fig. 4-2, a vertex $\mathrm{z}$ cut at $\pm 8 \mathrm{~cm}$ would be sufficient to remove the major part of endcap contamination. To deal with the non-Gaussian tail of the endcap distribution that extends into the gas body, we scaled the charge-normalized yield from entrance and exit windows of the empty cell (separately, with a cut at L.tr.vz=0) by the measured window thickness (see table 4.2.3) of each target cell. Then we subtracted the scaled empty cell contribution from the yield of each gas cell in every $x_{b j}$ bin. The Dummy target data were used for L17-SRC2 and EP kinematics where we didn't accumulate enough statistics with empty cell. This endcap subtraction method assumes that the gas cells and the dummy foils are aligned. We checked the cell alignment by fitting the peaks of entrance and exit windows in the yield distribution with Gaussian functions. The peak values are $-0.1185 \pm 0.0005 \mathrm{~m}$ and $0.1341 \pm 0.0005 \mathrm{~m}$. That is consistent with the target center of $6.5 \mathrm{~mm}$ from the pointing

\begin{tabular}{llc}
\hline Sources & Types & uncertainty \\
\hline Beam Energy & correlated & $0.005 \%$ \\
\hline Scattering Angle & correlated & $0.8 \mathrm{mrad}$ \\
\hline Momentum & correlated & $1.5 \mathrm{MeV}$ \\
\hline Charge & normalization & $0.22 \mu \mathrm{A}$ \\
\hline Tracking Efficiency & uncorrelated & $0.2-0.5 \%$ \\
\hline Trigger Efficiency & uncorrelated & 0 \\
\hline Gas Target Density Correction & normalization & $1 \%$ \\
\hline
\end{tabular}

Table 4.1: List of systematic uncertainties in the extracted yield. 
study 3.2.1. As shown in Fig. 4-3, the endcap contamination level increased from $1 \%$ at $x_{b j}=1$ to $10 \%$ at $x_{b j}=2.5$. A $30 \%$ correlated systematic uncertainty is assigned to this process due to the uncertainty on the window thickness.
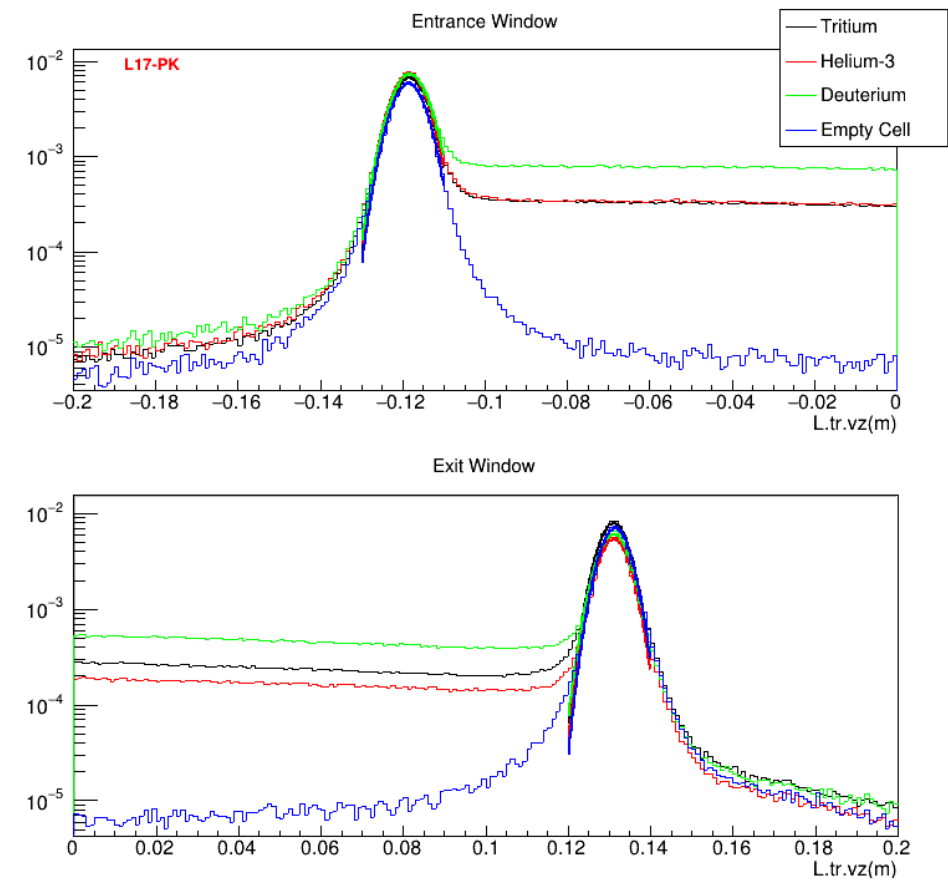

Figure 4-2: Gaussian fits of entrance (top) and exit (bottom) window yield distributions along vertex $z$ for various targets at L17-PK kinematics. Note that the yield is normalized by charge and also the quoted window thickness. Though a significant difference in amplitude is shown between the exit windows of the empty cell and dummy target, their integrals between $-8 \mathrm{~cm}<z<0$ agree within $30 \%$.

\subsubsection{Beam-induced Gas Target Density Change}

The beam deposits heat on the gas body as well as the entrance and exit windows. As a consequence, the gas is no longer uniformly distributed in the target cell, and the area density seen by the beam (hereafter referred as the effective density) is reduced. As shown in Fig. 4-4, the yield hence the target area density decreases with the beam current. It's 


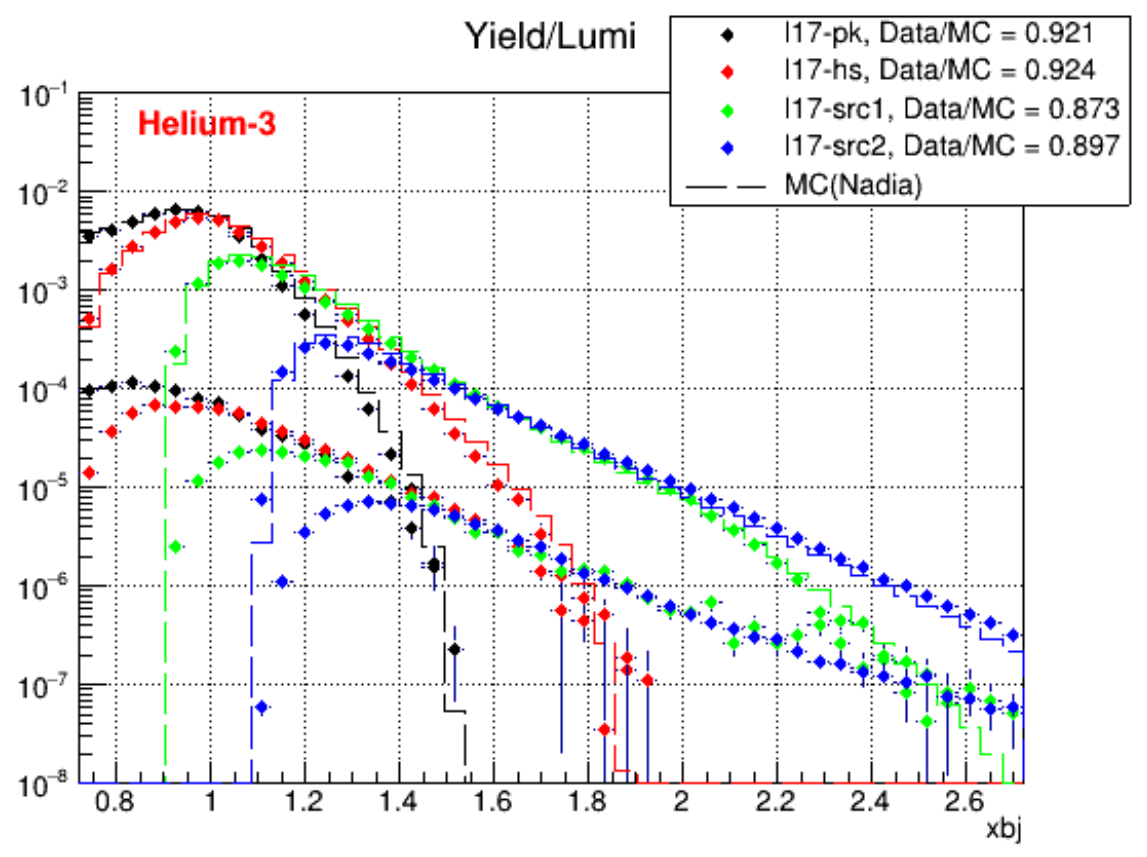

Figure 4-3: Helium-3 yield with respect to $x_{b j}$ from four overlapped kinematics at 17 degrees. The lower group of markers are the total endcap contributions. Dashed lines are MonteCarlo simulations, see Sec. 4.3 for details.

important to know:

1. with a stable beam, how soon can the gas in the cell reach the equilibrium state,

2. what is the relation between the beam current and the effective gas density,

3. is there any long-term density change (i.e. after hours of beam).

Simulations show the sudden change of gas density happens within the first 2 seconds of beam [62]. During the experiment, beam current ramps up from $0 \mu \mathrm{A}$ to $22.5 \mu \mathrm{A}$ at a rate of $1 \mu \mathrm{A} / \mathrm{s}$. No obvious change in the charge-normalized yield is observed from data once the beam current is stabilized [63]. A careful study of the beam current to gas density relation has been done by parameterizing the relative yield of the gas target as a second order function of the beam current 64. The result shows that with a $22.5 \mu \mathrm{A}$ beam, the tritium and helium-3 density decreased by $9 \%$ and $6 \%$ respectively. A $1 \%$ correlated systematic 
uncertainty on the target density is quoted for this correction.

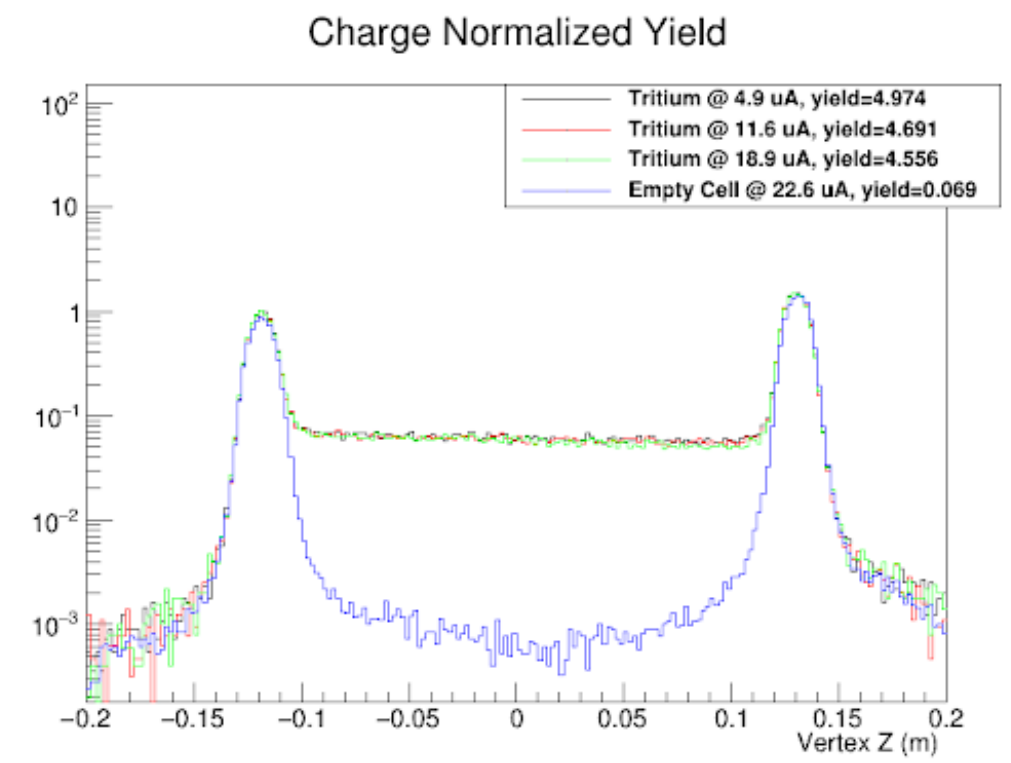

Figure 4-4: Yield distributions from gas targets and empty cell along vertex $z$ at L17-PK. Numbers shown on the legend are the integrated yield between $-8 \mathrm{~cm}<z<8 \mathrm{~cm}$.

\subsubsection{Hydrogen Contamination in the Second Tritium Cell}

During the Fall 2018 run period, we noticed in our QE data that the Tritium target produced a sharp peak at $x_{b j}=1$ with the same shape and $1.6 \%$ of the strength as the hydrogen elastic spectrum. Though there is no conclusion on this hydrogen contamination, a very reasonable hypothesis [65] is that when the cell was filled, some water residual adhered to the target cell wall, and released hydrogen through the reaction $\mathrm{H}_{2} \mathrm{O}+\mathrm{T}_{2} \rightarrow H T O+H T$. The HTO molecule stayed on the wall but the $H T$ gas was mixed with our tritium gas. In this case 
we have to know the tritium density loss as it was replaced by hydrogen atoms:

$$
\begin{aligned}
\rho_{\text {loss }}^{3 H} & =1.6 \% \cdot \rho^{H} \cdot M^{3 H} / M^{H} \\
\Rightarrow & \rho_{\text {real }}^{3 H}=\rho_{0}^{3 H}-\rho_{\text {loss }}^{3 H}=\rho_{0}^{3 H}-3 \cdot 1.6 \% \cdot \rho^{H} .
\end{aligned}
$$

This gives $\rho_{\text {real }}^{3 H}=0.8136 \mathrm{~g} / \mathrm{cm}^{2}$, a $4 \%$ decrease from the quoted density. This tritium cell was sent back to its manufacturer. A spectroscopy study is scheduled to study the gas content. For now a $2 \%$ density reduction is assumed with a $\pm 2 \%$ normalization uncertainty.

The hydrogen contamination needs to be subtracted from the tritium QE data. And it should not have any impact on the $x_{b j}>1$ physics since the hydrogen cross section dies off fast beyond its elastic peak.

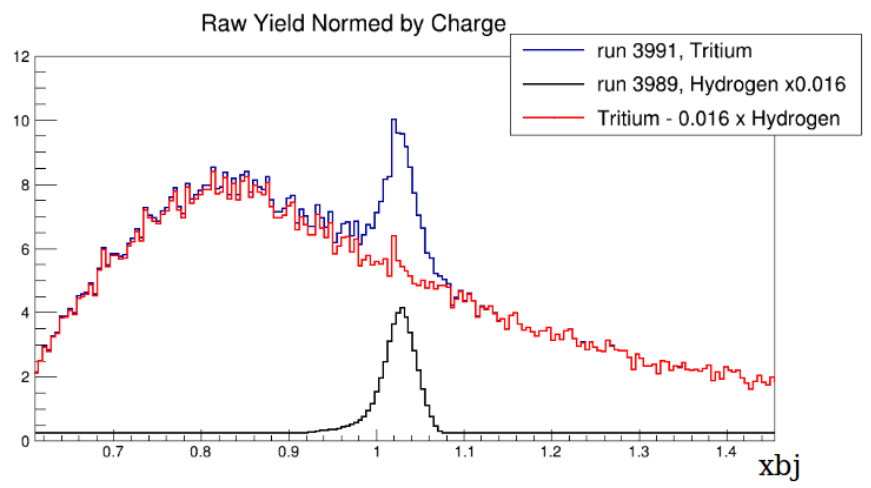

Figure 4-5: Subtracting the hydrogen contamination peak with real hydrogen elastic data at the same kinematics.

\subsubsection{Tritium Decay}

Tritium decays to helium-3 through the process ${ }^{3} H \rightarrow{ }^{3} H e+e^{-}+\bar{\nu}_{e}$ with a half-life of $t_{1 / 2}=\tau \cdot \ln 2=4500 \pm 8$ days (12.3 years). 


\begin{tabular}{|l|l|l|}
\hline Tritium Cells & Cell \#1 & Cell \#2 \\
\hline Date of Filling & $10 / 22 / 2017$ & $08 / 24 / 2018$ \\
\hline Initial Tritium Density (g/cm2) & 0.0851 & 0.0851 \\
\hline Initial Deuterium Density(\%) & 0.031 & 0.025 \\
\hline Initial Helium-3 Density(\%) & 0.030 & 0.014 \\
\hline Tritium Density Loss to Hydrogen (\%) & 0 & $2 \pm 2 \%$ \\
\hline Days from filling to SRC data taken & $174-194$ & $34-66$ \\
\hline Fraction of Tritium remains & $0.962-0.959$ & $0.992-0.985$ \\
\hline
\end{tabular}

Table 4.2: Tritium target density details.

Assume that the tritium cell contained $\rho_{T, 0}^{3 H}$ of tritium and $\rho_{T, 0}^{3 H e}$ of helium-3 at the time of filling, where $\rho$ is the area density in $\mathrm{g} / \mathrm{cm}^{2}$. After $t$ days, the cell contains:

$$
\begin{aligned}
\rho_{T}^{3 H}(t) & =\rho_{T, 0}^{3 H} f(t), \\
\rho_{T}^{3 H e}(t) & =\rho_{T, 0}^{3 H e}+\rho_{T, 0}^{3 H}[1-f(t)],
\end{aligned}
$$

where $f(t)=1-e^{-t / \tau}$ is the fraction of tritium remained after $t$ days of decay, and the initial helium-3 gas density in the tritium cell is reported as 0 . Note that the second equation only holds up to the assumption that tritium and helium-3 have the same molar mass. The gas density in the helium-3 cell does not change over time: $\rho_{H}^{3 H e}(t) \equiv \rho_{H, 0}^{3 H e}$. Then the measured yield ratio of tritium to helium-3 satisfies:

$$
\begin{aligned}
& \frac{\left[\rho_{0}^{3 H} Y^{\text {meas }}\right]_{T}}{\left[\rho_{0}^{3 H e} Y^{\text {meas }}\right]_{H}}=\frac{\left[\rho_{T}^{3 H}(t) Y^{3 H}+\rho_{T}^{3 H e}(t) Y^{3 H e}\right]}{\rho_{H}^{3 H e} Y^{3 H e}} \\
\Rightarrow & \frac{Y^{3 H}}{Y^{3 H e}}=\frac{1}{f(t)}\left[\frac{Y_{T}^{\text {meas }}}{Y_{H}^{\text {meas }}}-(1-f(t))\right]
\end{aligned}
$$


where $\frac{Y^{3 H}}{Y^{3 H e}}$ is the ratio of extracted tritium and helium-3 yield which is equivalent to $\frac{\sigma^{3 H}}{\sigma^{3 H e}}$ (see Sec. 4.5. In the same way, the $\frac{\sigma^{3 H}}{\sigma^{2 H}}$ ratio was corrected with the $\frac{\sigma^{3 H e}}{\sigma^{2 H}}$ results:

$$
\frac{Y^{3 H}}{Y^{2 H}}=\frac{1}{f(t)}\left[\frac{Y_{T}^{\text {meas }}}{Y_{D}^{\text {meas }}}-(1-f(t)) \frac{Y_{H}^{\text {meas }}}{Y_{D}^{\text {meas }}}\right]
$$

More information about the tritium decay are listed in Table 4.2.3. In this analysis, $f(t)=$ $0.960 \pm 0.002$ and $0.988 \pm 0.003$ were applied to the spring and fall data respectively.

\subsection{Monte-Carlo Simulation}

\subsubsection{Phase-space Generator}

The Hall A Single Arm Monte-Carlo simulation package [66] was used to generate phasespace distributions, that is, $\theta_{t g}, \phi_{t g}, \delta_{t g}$, and $y_{t g}$ on the target plane for each kinematics. This Fortran code randomly generate events within given range of position, momentum, and angles to simulate a scattered electron, then transports electrons through spectrometer magnets and apertures step-by-step to reach the focal plane, and reconstruct back to target plane. Only successfully reconstructed events were recorded in rootfiles. Transportation matrices were generated by COSY INFINITY V8.1 [67]. The dimension and field information of each HRS magnet from [68] were provided as inputs to generate a corresponding mapping (forward matrix) of the track position and momentum between the entrance and exit of that magnet. The backward (reconstruction) matrix is a numerical inverse of the product of all forward matrices between the entrance of Q1 and the exit of Q3. The simulation was updated and tuned for new Q1 magnets during the $G_{M}^{p}$ experiment. Multiple scattering, electron energy loss, and drift chamber resolutions are implemented to match 
spectrometer resolutions between the data and simulation.

\subsubsection{Cross Section Model}

The inclusive cross section of each simulated event was calculated with the XEMC inclusive cross section package [69]. $\mathrm{XEMC}$ is a $\mathrm{C}++$ wrapper of several cross section models at different kinematic ranges: an inelastic piece from F1F209 empirical fitting [70] and the y-scaling model for QE contribution (see Sec. 1.3.1). The radiative correction calculation was based on the Mo and Tsai's approach [71], except that in the internal corrections an equivalent radiator was used to account for materials before and after the target [72].

Experimentally the scaling function $F(y)$ can be parameterized with five variables. For Deuterium

$$
F(y)=\left(f_{0}-B\right) \cdot \frac{\alpha^{2} e^{-(a y)^{2}}}{\alpha^{2}+y^{2}}+B e^{-b|y|},
$$

For heavier nuclei

$$
F(y)=\left(f_{0}-B\right) \cdot \frac{\alpha^{2} e^{-(a y)^{2}}}{\alpha^{2}+y^{2}}+B e^{-(b y)^{2}},
$$

with $y$ solved from

$$
M_{A}+\nu=\sqrt{M^{2}+(y+q)^{2}}+\sqrt{M_{A-1}^{* 2}+y^{2}} .
$$

$M_{A}, M, M_{A-1}^{*}$ are the mass of the initial nucleus, scattered nucleon, and recoiling $(A-1)$ system respectively.

In the first pass of data to simulation comparison we used $f_{0}, B, a, b, \alpha$ parameters from 


\begin{tabular}{lllll}
\hline Name & ${ }^{2} \mathbf{H}$ & ${ }^{3} \mathbf{H}$ & ${ }^{3} \mathbf{H e}$ & $\mathbf{C}$ \\
\hline $\mathrm{A}$ & 2 & 3 & 3 & 12 \\
\hline $\mathrm{Z}$ & 1 & 1 & 2 & 6 \\
\hline Mass (Amu) & 2.014 & 3.016 & 3.016 & 12.011 \\
\hline $\begin{array}{l}\text { Radiation Length } \\
\text { (g/cm2) }\end{array}$ & 122.6 & 183.9 & 71.1 & 42.7 \\
\hline $\begin{array}{l}\text { Separation Energy } \\
\text { (MeV) }\end{array}$ & 2.250 & 6.257 & 5.493 & 17.270 \\
\hline f0 & 8.742 & 5.309 & 5.309 & 2.4112 \\
\hline $\mathrm{B}$ & 0.8239 & 2.184 & 2.184 & 0.6915 \\
\hline $\mathrm{a}$ & 7.727 & 2.886 & 2.886 & 3.1128 \\
\hline $\mathrm{b}$ & 9.394 & 10.35 & 10.35 & 6.7769 \\
\hline alpha & 45.3 & 64.2 & 64.2 & 161.88 \\
\hline
\end{tabular}

Table 4.3: $F(y)$ fitting parameters and other informations used in the XEMC cross section model.

the JLAB experiment E02-019 [72] for deuterium and helium-3, and parameters from experiment E08-014 [11] for Carbon. There are no tritium QE data at similar kinematics, so we used the helium-3 parameters for tritium. The fitting parameters, the separation energy which is required to calculate $y$, as well as other quantities used in the cross section calculation are listed in Table 4.3 . Later new fittings were performed with cross sections from this thesis to update the parameters for deuterium, tritium and helium-3.

The QE cross section is calculated from $F(y)$ :

$$
\sigma_{Q E}=F(y)\left(Z \widetilde{\sigma}_{p}+N \widetilde{\sigma}_{n}\right)\left(\frac{q}{\sqrt{M^{2}+(y+q)^{2}}}\right)^{-1}
$$

where $\widetilde{\sigma}_{p(n)}$ is the electron-nucleon cross section of an off-shell proton (neutron).

\subsubsection{Data to Simulation Comparison}

For each kinematic settings, $N_{t o t}=10^{7}$ Monte-Carlo events are generated within the range of $-100 \mathrm{mrad}<\phi_{t g}<100 \mathrm{mrad},-100 \mathrm{mrad}<\theta_{t g}<100 \mathrm{mrad}$, and $-10.0 \%<\delta_{t g}<10.0 \%$ 
to cover the entire HRS acceptance. Then every successfully-reconstructed simulation event is weighted by the radiative cross section from XEMC to get the yield-per-luminosity

$$
Y_{M C}=\frac{\Omega_{t o t} \Delta E^{\prime}}{N_{t o t}} \sum_{N_{t o t}} A\left(E_{i}^{\prime}, \theta_{i}\right) \frac{d \sigma\left(E_{i}^{\prime}, \theta_{i}\right)}{d \Omega d E^{\prime}}
$$

where $\left(E_{i}^{\prime}, \theta_{i}\right)$ represents the scattered electron momentum and angle for the $i_{t h}$ event, $\Omega_{t o t}$ and $\Delta E^{\prime}$ are solid angle and momentum range covered by the simulation, and $A\left(E_{i}^{\prime}, \theta_{i}\right)$ is the discrete acceptance function which equals 1 if the event is successfully reconstructed back to target plane and 0 otherwise.

The yield from the Monte-Carlo simulation is compared with the yield from data after corrections to study the acceptance and bin centering effect, and also to extract the absolute cross section.

\subsubsection{Acceptance Cuts}

The data to simulation comparison with carbon foil at each kinematics was used as a sanity check. Since the carbon cross section model was improved by previous experiment to match data at similar kinematics, and the carbon foil locates at the center of $y_{t g}$ where the HRS acceptance is well-understood, its data and simulation should have good agreement. As shown in Fig. 4-6, with loose acceptance cuts $\left(-4.0 \%<\delta_{t g}<4.0 \%,-30 \mathrm{mrad}<\phi_{t g}<30\right.$ $\operatorname{mrad}$, and $\left.-60 \mathrm{mrad}<\theta_{t g}<60 \mathrm{mrad}\right)$ the data and simulation yield distribution agree in shape and magnitude except a small difference in the out-of-plane angle $\left(\theta_{t g}\right)$ distribution. Same comparison with helium-3 gas cell (see Fig. 4-7) shows that the acceptance in the extended vertex $z$ range of $-8 \mathrm{~cm}<$ L.tr.vz $<8 \mathrm{~cm}$ is also under control. So we confirmed that the "loose" cuts are good for the cross section ratio analysis.

For the absolute cross section analysis, more strict acceptance cuts $\left(-3.5 \%<\delta_{t g}<3.5 \%\right.$, 

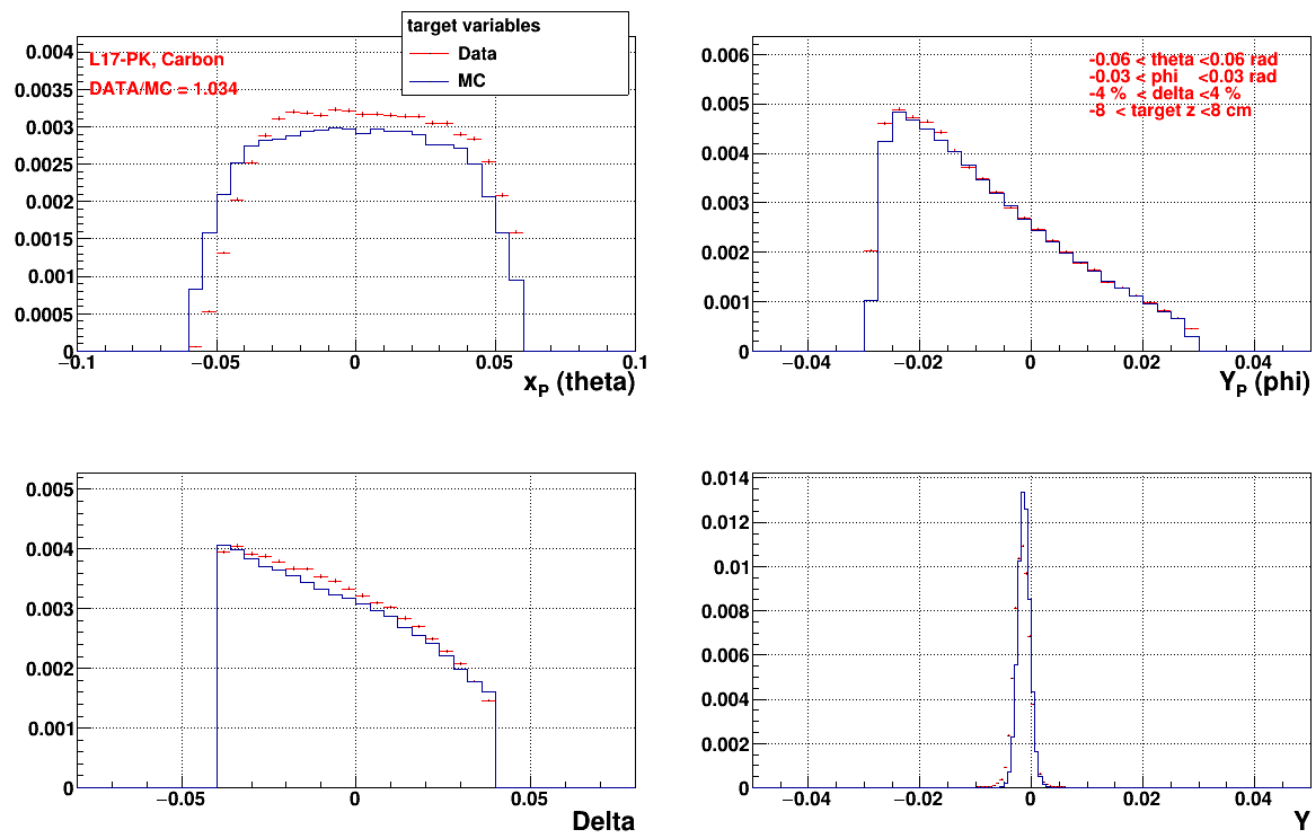

Figure 4-6: Data to Monte-Carlo simulation yield comparison with the carbon foil at L17PK kinematics with loose acceptance cuts. The integrated data to Monte-Carlo yield ratio is 1.034 .

$-25 \mathrm{mrad}<\phi_{t g}<25 \mathrm{mrad}$, and $-40 \mathrm{mrad}<\theta_{t g}<40 \mathrm{mrad}$ ) were applied to remove the data to simulation discrepancies at the falling edge of acceptances. With this group of "tight" cuts, the agreement at the edge of acceptance was significantly improved (see Fig. 4-8).

\subsubsection{Binning}

The target variable $\delta_{t g}$ hence the scattered electron momentum $E^{\prime}$ is a natural choice of binning for initial yield analysis, which includes the data to Monte-Carlo comparison, endcap contaminations check, and overlapped kinematics check as shown in Fig. 4-9, Then the cross section (ratios) need to be extracted as $\frac{d \Omega}{d x d Q^{2}}$ to study the scaling behavior at SRC region.

The acceptance defined by the spectrometer is almost a rectangle in scattering angle and 

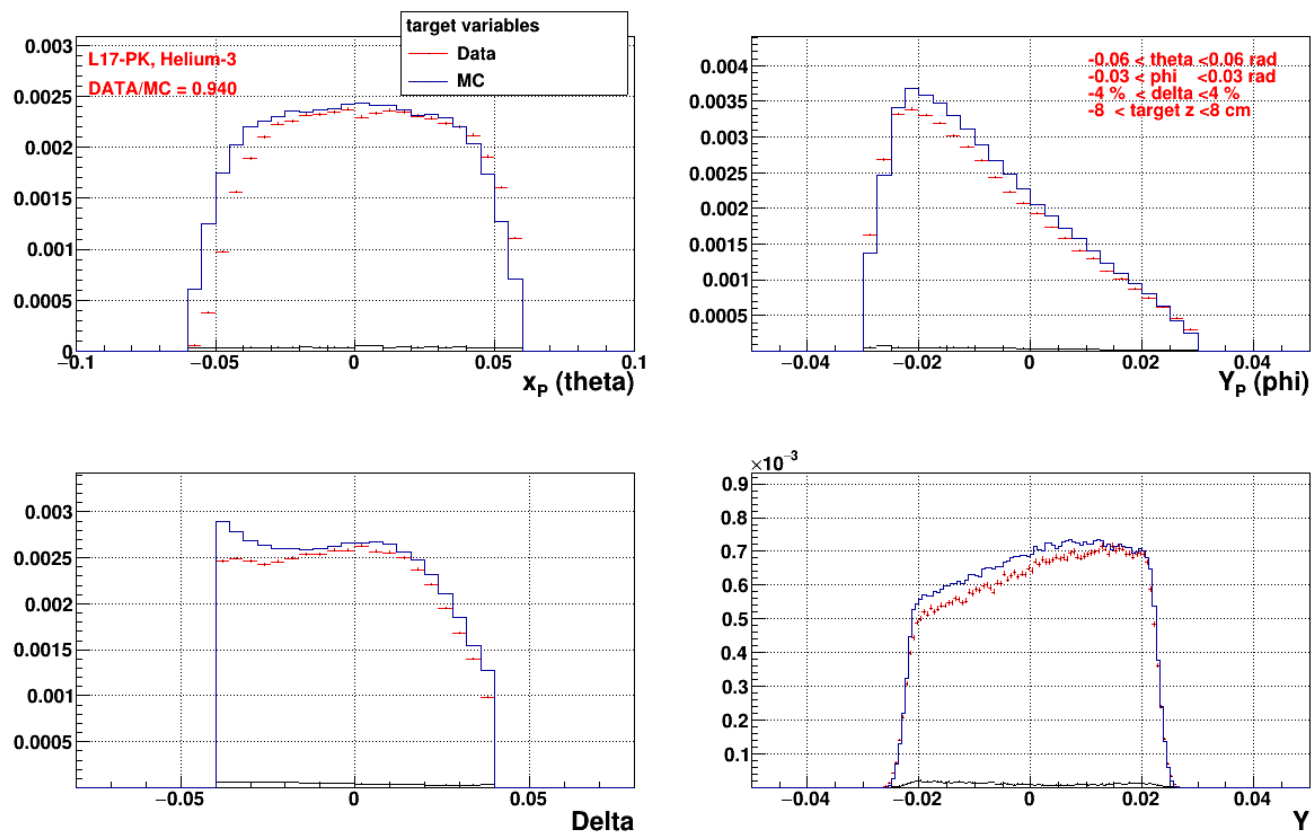

Figure 4-7: Data to Monte-Carlo simulation yield comparison with helium-3 target at L17PK kinematics with loose acceptance cuts and vertex $z$ cut. The integrated data to MonteCarlo yield ratio is 0.940 .

$E^{\prime}$ as shown in Fig. 4-11. But each $E^{\prime}$ bin contains events from a wide range of $x_{b j}$. Since the cross section falls rapidly at $x_{b j}>1$, the mean value of an $E^{\prime}$ bin always represents events from the smaller $x_{b j}$ side of the bin. To avoid the large bin-centering correction and to make the result sensitive to the cross section at large $x_{b j}$ where the SRC contribution dominates, the yield was binned in $x_{b j}$ directly to extract cross section (ratios) instead of binning in $E^{\prime}$ then translated to $x_{b j}$. Yield from four overlapped L17 kinematics was shown in Fig. 4-10.

The $x_{b j}$ resolution

$$
\delta x_{b j}=\frac{\partial x_{b j}}{\partial E} \delta E+\frac{\partial x_{b j}}{\partial E^{\prime}} \delta E^{\prime}+\frac{\partial x_{b j}}{\partial \theta} \delta \theta
$$



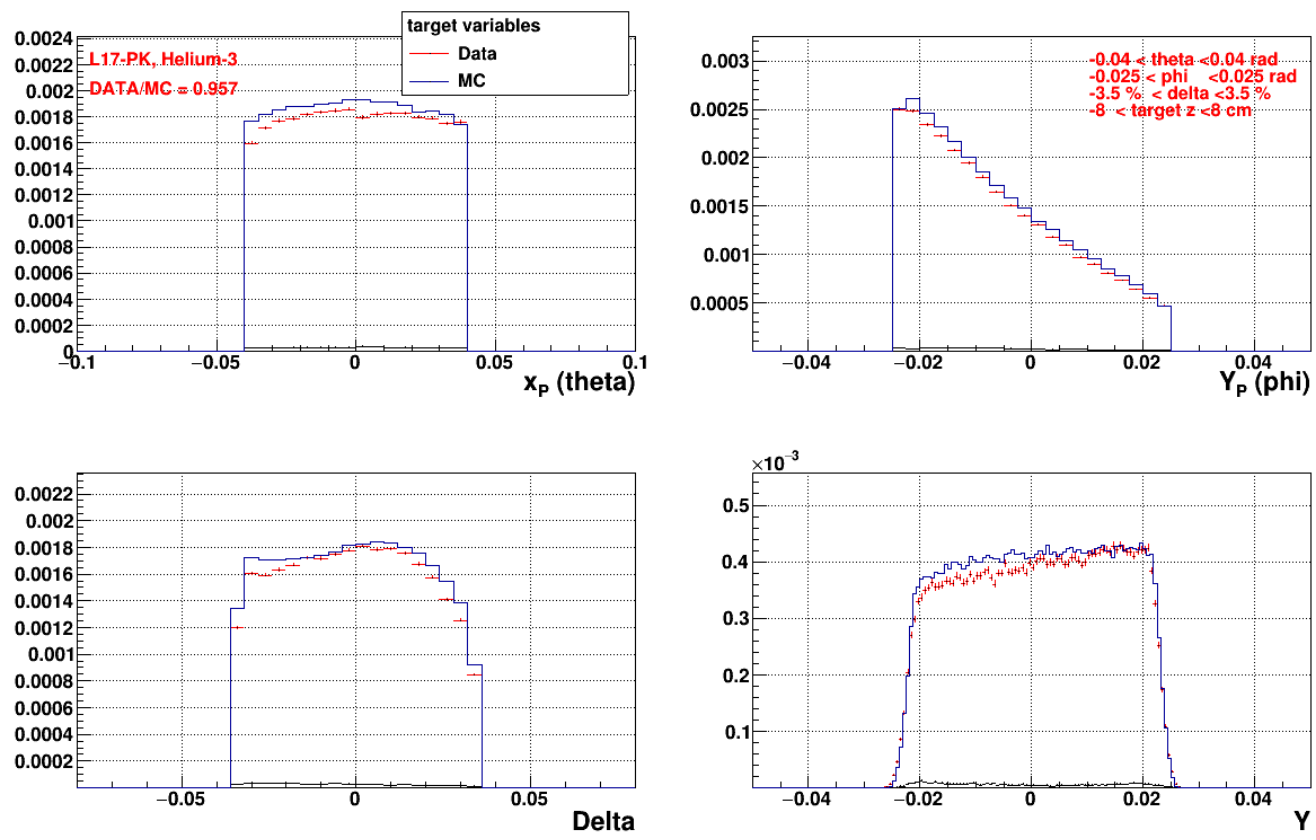

Figure 4-8: Data to Monte-Carlo simulation yield comparison with helium-3 target at L17PK kinematics with tight acceptance cuts and vertex $z$ cut. The integrated data to MonteCarlo yield ratio is 0.957 .

where $\theta$ is the scattering angle. Fig. 4-12 shows the $x_{b j}$ resolution at L17 kinematics calculated from the uncertainties provided in Table 2-19, Based on this result, the $x_{b j}$ bin size of $0.025,0.05$, and 0.1 were chosen for $\mathrm{QE}, x_{b j}>1$, and $x_{b j}>2$ kinematics respectively.

\begin{tabular}{cc}
\hline Sources & Resolution \\
\hline Beam Energy & $0.005 \%$ \\
\hline Scattering Angle & \\
\hline$y_{t g}$ & $1.5 \mathrm{mrad}$ \\
\hline$\theta_{t g}$ & $1.0 \mathrm{mrad}$ \\
\hline$\phi_{t g}$ & $0.5 \mathrm{mrad}$ \\
\hline Momentum & $0.25 \mathrm{e}-3$ \\
\hline
\end{tabular}

Table 4.4: List of resolutions related to $x_{b j}$. The $y_{t g}$ resolution is from optics calibration. Angle and momentum resolutions are from 2-19 

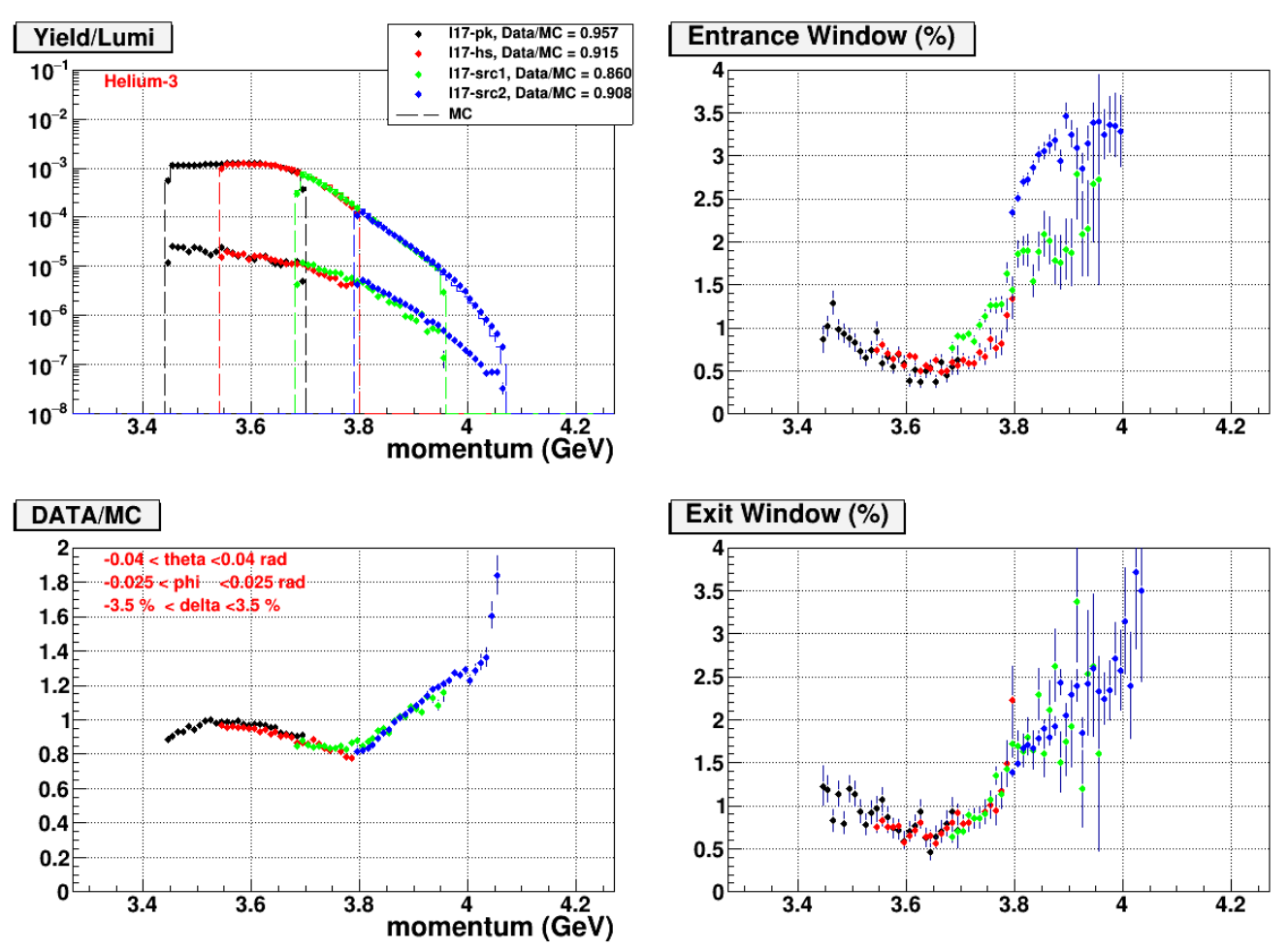

Figure 4-9: Top left: Data to Monte-Carlo simulation yield comparison with helium-3 target at four overlapped L17 kinematics with respect to the scattered electron momentum $E^{\prime}$. Dashed lines represent yield from simulation. Lower markers are contributions from endcaps. Bottom Left: data to simulation ratio. Top (bottom) right: bin-by-bin endcap contamination from the target entrance (exit) window with respect to $E^{\prime}$. The discrepancy between L17-SRC1 and L17-SRC2 entrance window contribution was absorbed into the $30 \%$ uncertainty in the endcap subtraction.

\subsection{Absolute Cross Section Extraction}

As shown in Fig. 4-13, the carbon data distribution agrees with the simulation in shape under tight cuts. That means the acceptance effect is well-understood within this acceptance range. Therefore we can treat the data to simulation yield ratio at the $i^{\text {th }} x_{b j}$ bin as the ratio of experimental cross section to the radiative cross section from XEMC model:

$$
\frac{Y_{\text {data }}^{i}}{Y_{M C}^{i}}=\frac{\sigma^{\text {data }}\left(x_{i}, Q_{i}^{2}\right)}{\sigma_{\text {rad }}^{\text {model }}\left(x_{i}, Q_{i}^{2}\right)},
$$



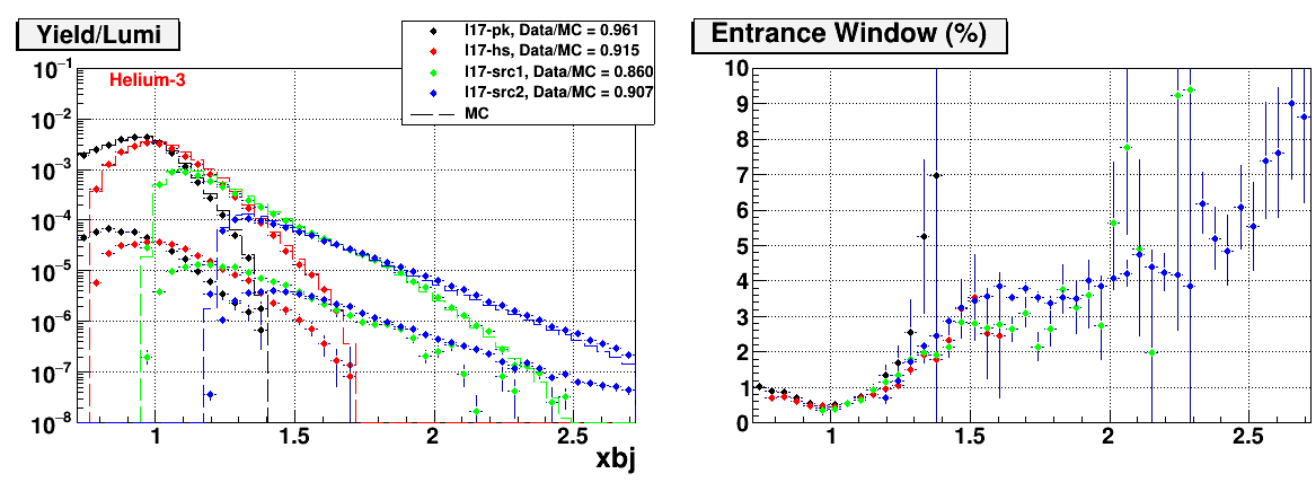

\section{DATA/MC}

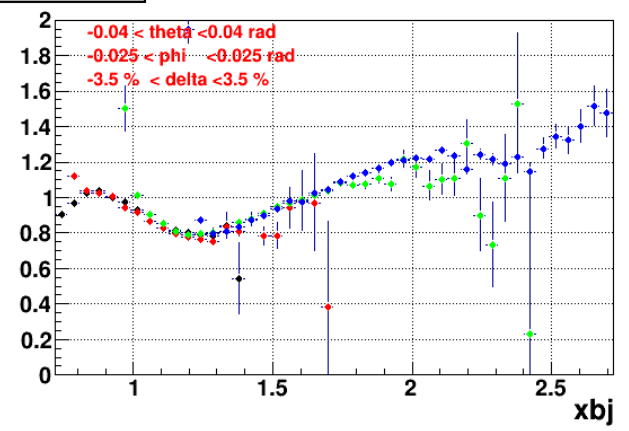

\section{Exit Window (\%)}

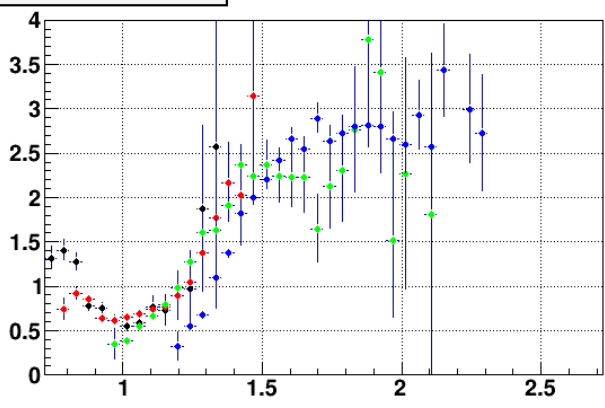

Figure 4-10: Data to Monte-Carlo simulation yield comparison, and endcap contamination of helium-3 target at four overlapped L17 kinematics with respect to $x_{b j}$.

To decide $x_{i}$ and $Q_{i}^{2}$ of each $x_{b j}$ bin, their distributions from data and simulation were compared in shape and mean value. As one can already learn from Fig. 4-9, the $x_{b j}$ distributions from data and simulation follow the same shape. So the integrated yield of each bin can be well-represented by the value at the bin center (average). Similarly, the $Q^{2}$ distribution of each $x_{b j}$ bin can be well-described by the mean value as shown in Fig. 4-14. The radiative correction was applied bin-by-bin as a correction factor calculated from the XEMC model. The extracted Born cross section is:

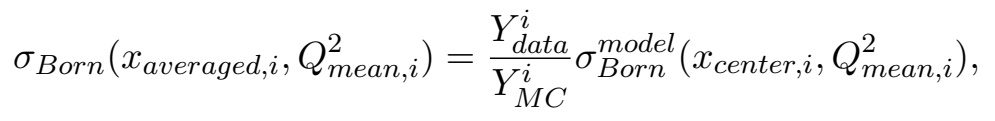




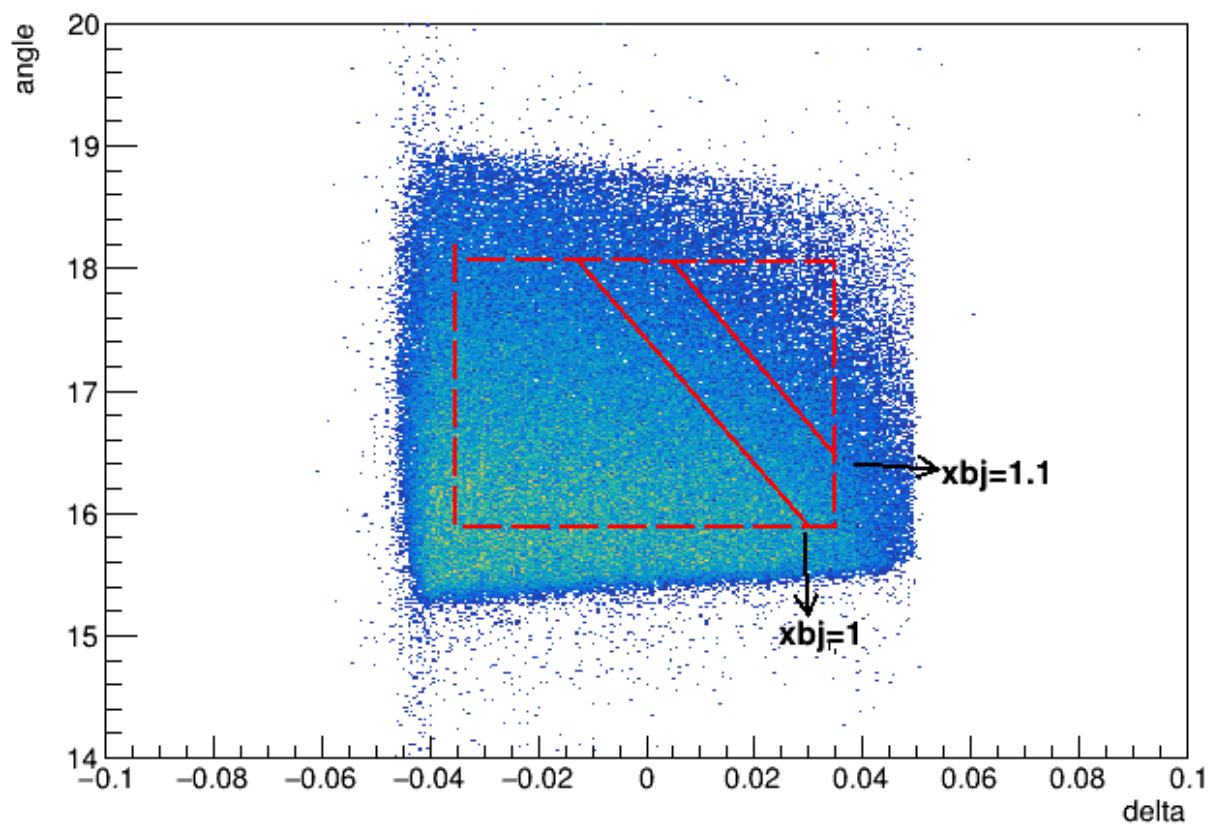

Figure 4-11: 2D Event distribution in scattering angle and momentum. Red box: tight acceptance cuts.

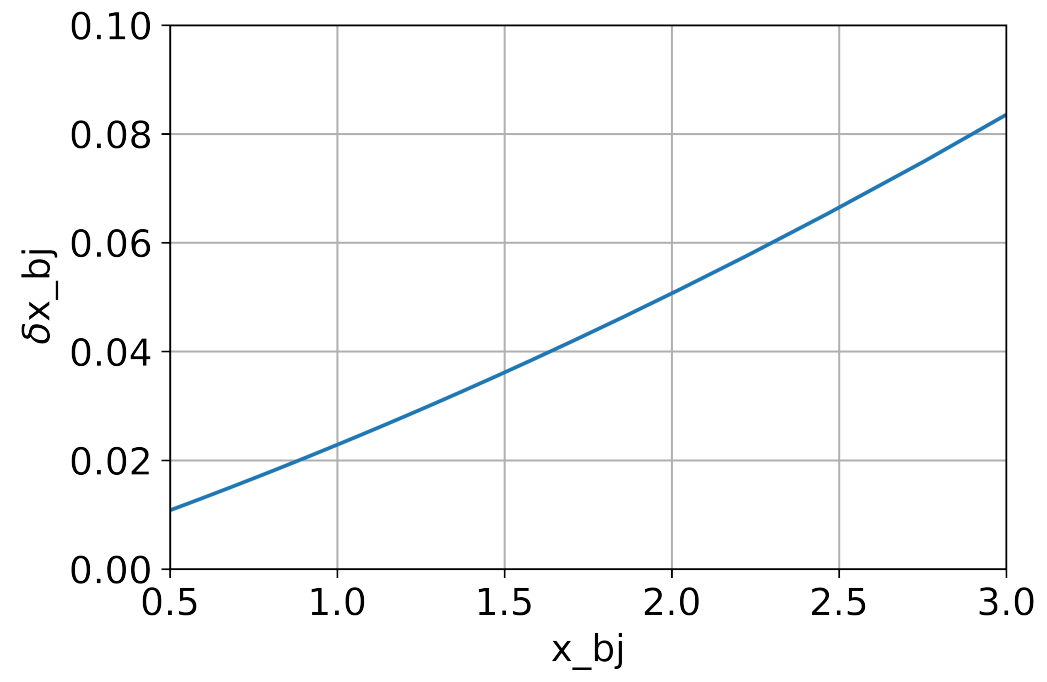

Figure 4-12: $x_{b j}$ resolution for L17 kinematics. Other settings have similar or higher resolutions 

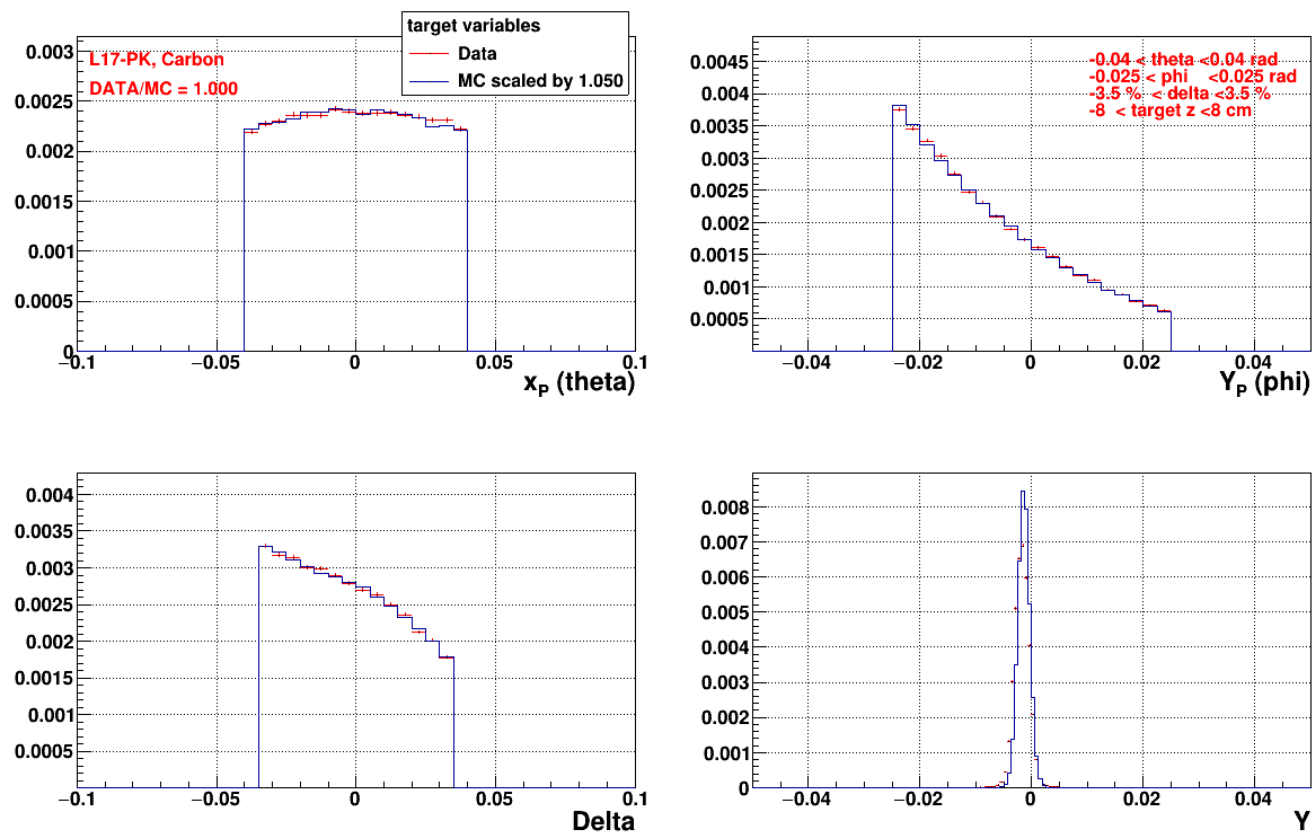

Figure 4-13: Data to Monte-Carlo simulation yield comparison of Carbon foil at L17-PK kinematics with tight acceptance cuts. The simulation is scaled by a factor of 1.05 to check the shapes of distributions.

\subsection{Cross Section Ratio Extraction}

After all necessary corrections as discussed in previous sections, the extracted per-nucleon yield $Y_{\text {data }}^{\text {cor }}$ from each gas target was compared with the simulation as a sanity check. At each kinematic settings, the acceptance effects from the three identical cells are expected to be cancelled in the cross section ratios. Therefore the radiative cross section ratios of two targets reduce to their yield ratios. Then the radiative effect is removed by the XEMC model to give the Born cross section ratio between nucleus $\mathrm{A}$ and $\mathrm{B}$ as:

$$
\frac{\sigma^{\text {Born }}(A)}{\sigma^{\text {Born }}(B)}=\frac{Y_{\text {data }}^{c o r}(A)}{Y_{\text {data }}^{\text {cor }}(B)} \frac{\operatorname{radcor}(A)}{\text { radcor }(B)}
$$

where $\operatorname{radcor}(A)=\sigma_{X E M C}^{\text {Born }}(A) / \sigma_{X E M C}^{r a d}(A)$ is the XEMC-calculated Born to Radiative cross section ratio of the nucleus A. the Though the absolute radiative correction estimated 

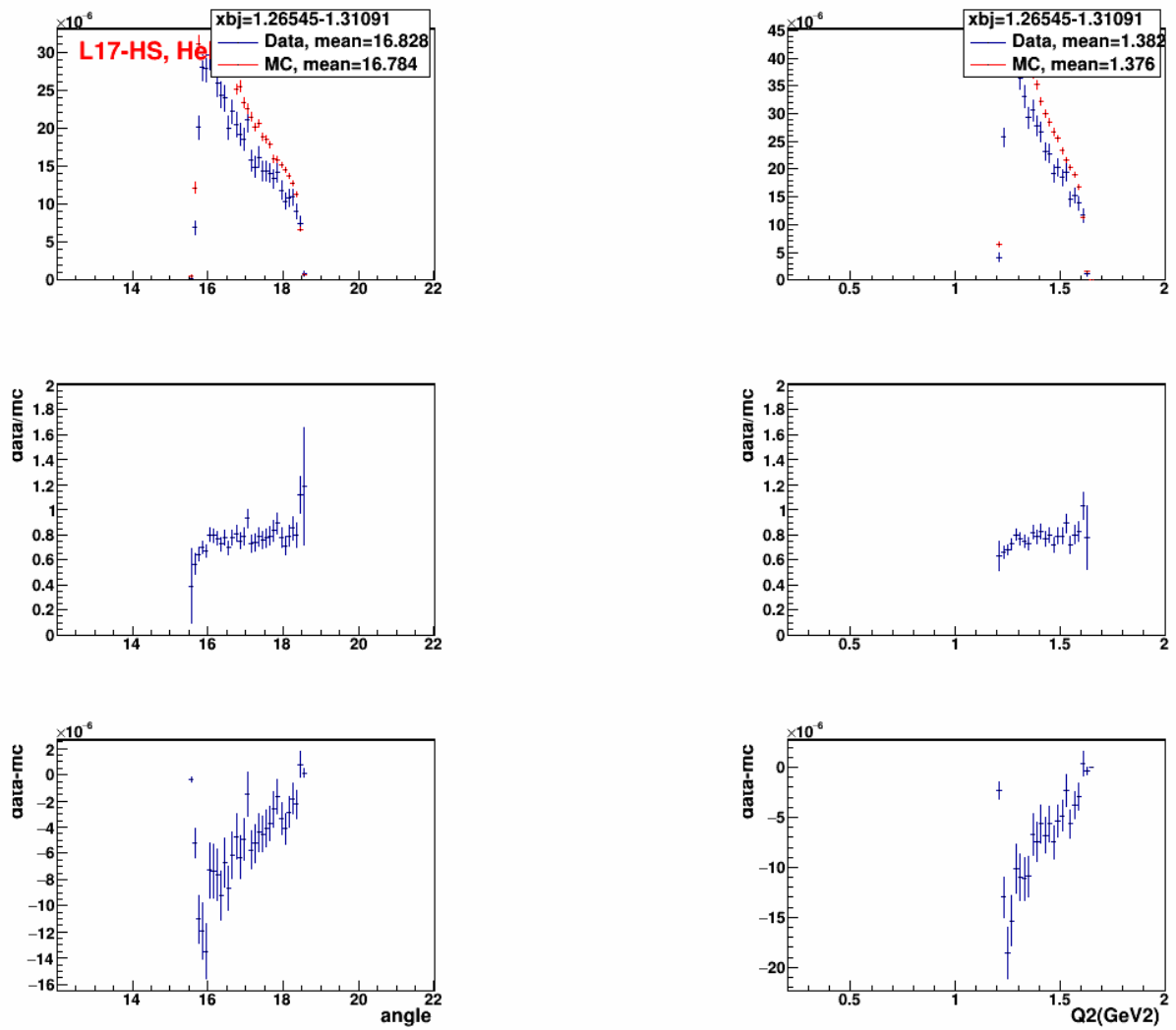

Figure 4-14: Compare the angle(left) and $Q^{2}$ (right) distribution within a given $x_{b j}$ bin. Despite a difference in amplitude, their ratios are flat, and their mean values are consistent within resolution.

by the XEMC model can be as large as $40 \%$ at $x_{b j}>1$, they are cancelled in the tritium to helium-3 cross section ratio (see Fig. 4-15). A 1\% point-to-point systematic uncertainty and another $1 \%$ normalization uncertainty are assigned to this process. 
Radiative correction factor from simulation
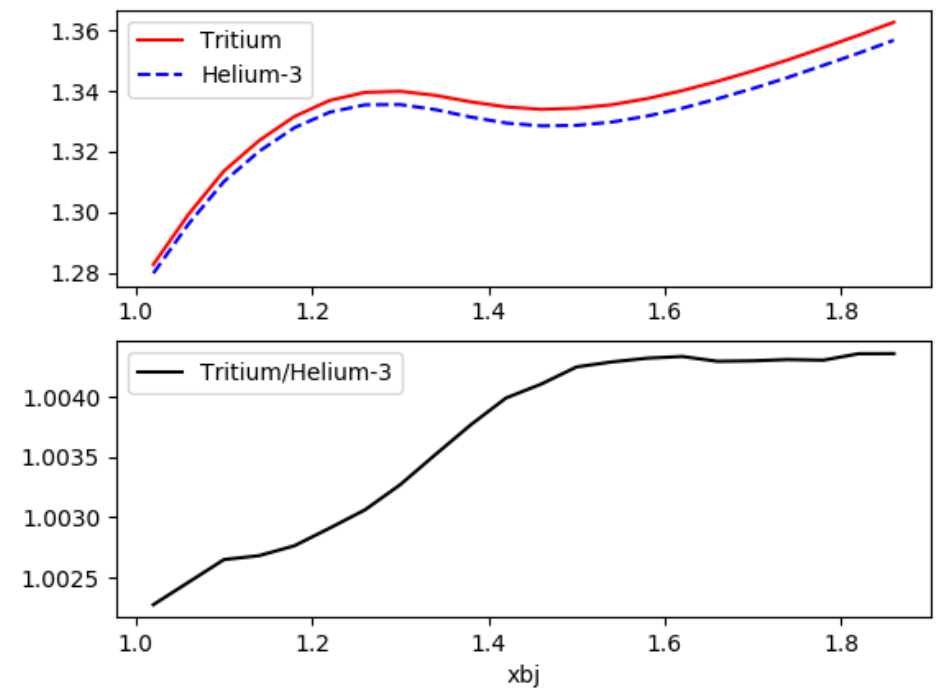

Figure 4-15: Top: the Born to radiative cross section ratio with tritium and helium-3 target. Bottom: the radiative correction factors from two targets agree within $0.5 \%$. 


\section{Chapter 5}

\section{Results and Discussions}

This chapter presents the ${ }^{3} \mathrm{H} /{ }^{3} \mathrm{He},{ }^{3} \mathrm{H} /{ }^{2} \mathrm{H}$, and ${ }^{3} \mathrm{He} /{ }^{2} \mathrm{H}$ cross section ratios, and ${ }^{2} \mathrm{H},{ }^{3} \mathrm{H}$, ${ }^{3} \mathrm{He},{ }^{12} \mathrm{C}$ absolute cross sections at $1<x_{b j}<3$ with a $Q^{2}$ range of 0.4 to 1.8 . This includes the LHRS data from L21, L24, L30, L17, EP kinematics. While the high $Q^{2}$ kinematics (L17 and EP) provided precise information on the isospin dependence of NN SRC pairs, kinematics from lower $Q^{2}$ are used to study the $Q^{2}$ dependence of the NN SRC plateau. The L21 and L17 kinematics also provide $x_{b j}>2$ cross sections and ratios that could be used in the $3 \mathrm{~N}$ SRC study.

The Cross section results are rebinned to the central angle so that values from overlapped kinematics with the same scattering angle can be combined. The $x_{b j}$ and $Q^{2}$ distributions are shown in Fig. 5-1.

\subsection{Systematic Uncertainties}

The beam, momentum, and angle uncertainties as listed in Table 4.1 are calculated with the XEMC model for each $x_{b j}$ bin. For example, $\sigma(E+\delta E) / \sigma(E)-1$ gives the beam energy uncertainty in absolute cross sections. Those uncertainties are correlated point-to-point uncertainties in extracted cross sections, and cancelled in ratios. To estimate the uncertainty from endcap subtraction, the contamination level is parameterized as a function which is 


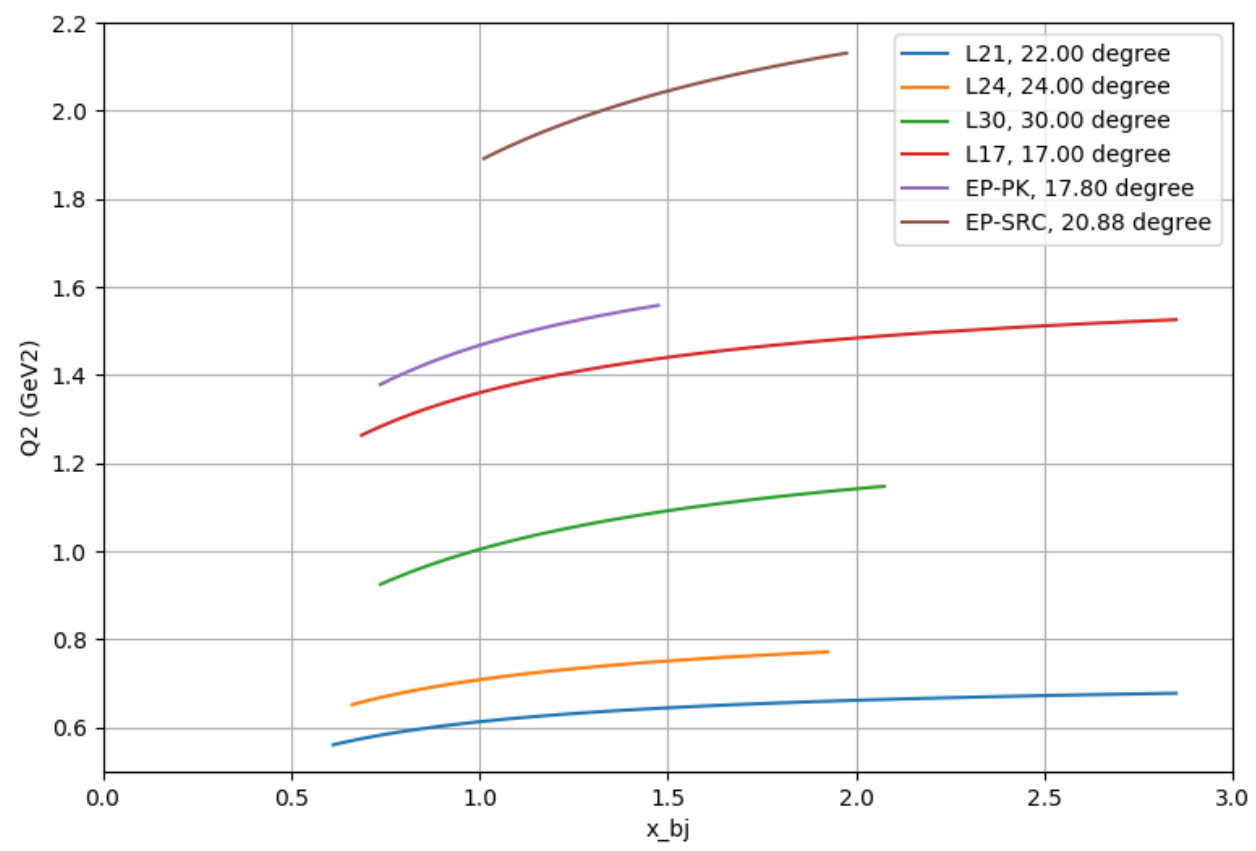

Figure 5-1: Extracted Tritium Born cross sections from various kinematics.

linear between $1<x_{b j}<3$ and symmetric about $x_{b j}=1.30 \%$ of the fitted contamination level enter the cross section as a correlated uncertainty. Same set of fitting parameters are used for deuterium, helium-3, and tritium data at the same kinematics. The beam charge uncertainty is $1 \%$ at 22.5 . It should be correlated with the $1 \%$ normalization uncertainty from the charge-induced gas target density correction. In this analysis we add them in quadrature as a safe overestimation. The charge uncertainty is cancelled in ratios but not the density correction. The radiative correction uncertainty in XEMC is estimated as a $1 \%$ normalization uncertainty and $1 \%$ uncorrelated point-to-point uncertainty according to the previous experiment [72]. This number will be updated in the future. The normalization part of the radiative correction uncertainty is cancelled in the ratio. For the L17 data taken with the second tritium cell, there is an additional $2 \%$ normalization uncertainty due to the density loss from the hydrogen contamination. 
To check the uncertainty from our choices of acceptance cuts, we varied the acceptance cuts
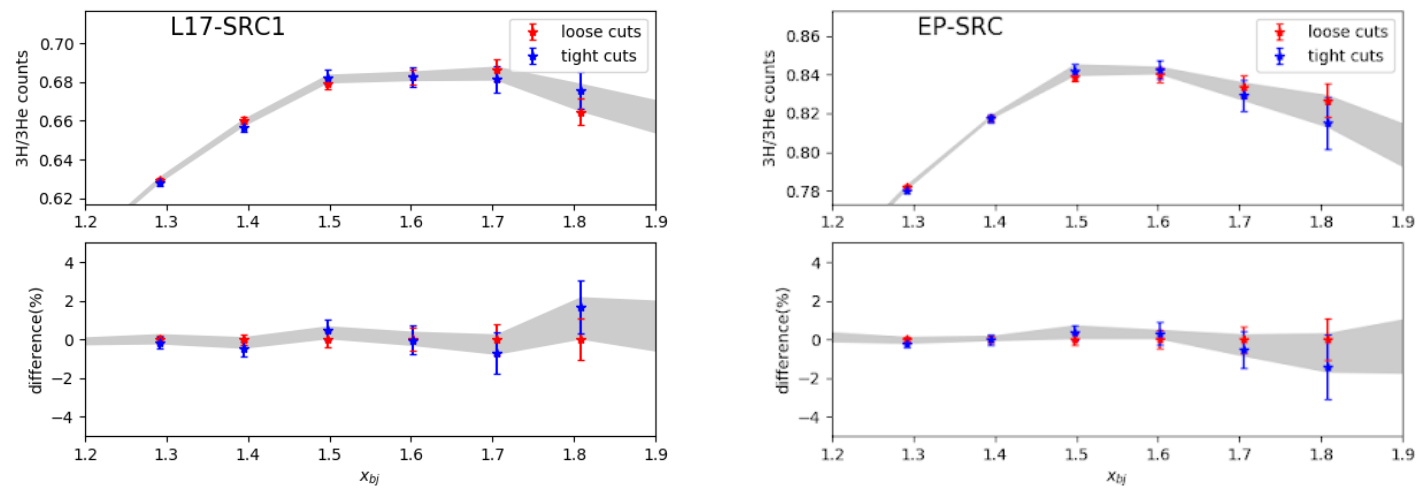

Figure 5-2: ${ }^{3} \mathrm{H} /{ }^{3} \mathrm{He}$ raw event counts ratio with different acceptance cuts and there difference in percentage with respect to the loose cuts(red) from kinematics L17-SRC1(left) and EP-SRC(right).

$(\theta, \phi, \delta)$ around the default values to study the change in results. For example, in Fig. 5-2 the ratio of ${ }^{3} \mathrm{H} /{ }^{3} \mathrm{He}$ raw counts (number of good electron events, not normalization by luminosity) is plotted with acceptance cuts over the range of:

- $-20 \mathrm{mrad}<\phi_{t g}<20 \mathrm{mrad} \longrightarrow-40 \mathrm{mrad}<\phi_{t g}<40 \mathrm{mrad}$,

- $-30 \mathrm{mrad}<\theta_{t g}<30 \mathrm{mrad} \longrightarrow-65 \mathrm{mrad}<\theta_{t g}<65 \mathrm{mrad}$,

- $-2.0 \%<\delta_{t g}<2.0 \% \longrightarrow-4.0 \%<\delta_{t g}<4.0 \%$.

The central values of corresponding ratio results are shown as shaded areas. The plots show no statistically-significant difference between loose and tight cuts. Similar study shows that the fluctuation of absolute cross section results is always within $40 \%$ of statistical uncertainties if varying the tight cuts by $\pm 5 \mathrm{mrad}$ in $\theta$ and $\phi$, or $0.3 \%$ in $\delta$. In practice a $1 \%$ uncorrelated uncertainty is assigned to cross section (ratios) at $1.7<x_{b j}<2.2$, and $2 \%$ at $x_{b j}>2.2$. 


\begin{tabular}{ccccc}
\hline Sources & Types & Uncert. & $\begin{array}{c}\text { Uncertainties } \\
\text { in Absolute } \\
\text { cross section }\end{array}$ & $\begin{array}{c}\text { Uncertainties } \\
\text { in Ratio }\end{array}$ \\
\hline Beam Energy & corr. & $0.005 \%$ & $0.1-0.5 \%$ & 0 \\
\hline angle & corr. & $0.8 \mathrm{mrad}$ & $0.1 \%-4.0 \%$ & 0 \\
\hline momentum & corr. & $1.5 \mathrm{MeV}$ & $0.1 \%-4.0 \%$ & 0 \\
\hline Acceptance cuts & uncor. & & $0-2 \%$ & $0-2 \%$ \\
\hline Endcap Contamination & cor. & $0.1 \%-3 \%$ & $0.1 \%-3.0 \%$ & $0.1 \%-4.2 \%$ \\
\hline Radiative Correction & uncor. & $1 \%$ & $1 \%$ & $1 \%$ \\
\hline Radiative Correction & norm. & $1 \%$ & $1 \%$ & 0 \\
\hline Tracking & norm. & $0.2-0.5 \%$ & 0 & 0 \\
\hline Charge & norm. & $0.2 \mu \mathrm{A}$ & $1 \%$ & 0 \\
\hline $\begin{array}{c}\text { Current-induced } \\
\text { Density Change }\end{array}$ & norm. & $1 \%$ & $1 \%$ & $1.4 \%$ \\
\hline $\begin{array}{c}\text { Tritium density Correction } \\
\text { (hydrogen contamination) }\end{array}$ & norm. & $2 \%$ & $2 \%$ & $2 \%$ \\
\hline
\end{tabular}

A full list of uncertainties are given in Table 5.1. An additional 1\% uncorrelated uncertainty is applied on all results to account for all other uncertainties of unknown sources. The cross section result has a total point-to-point uncertainty of $1.0 \%-6.5 \%$ with $1.7 \%$ normalization (2.7\% for the L17 tritium data). The cross section ratio result has a total point-to-point uncertainty of $1.0 \%-4.8 \%$ with $1.4 \%$ normalization ( $2.5 \%$ for the $\mathrm{L} 17$ tritium-related ratio data).

\subsection{Absolute Cross Sections}

The extracted absolute cross sections from deuterium, tritium, and helium-3 data are shown in Fig. 5-3 to Fig 5-5. 


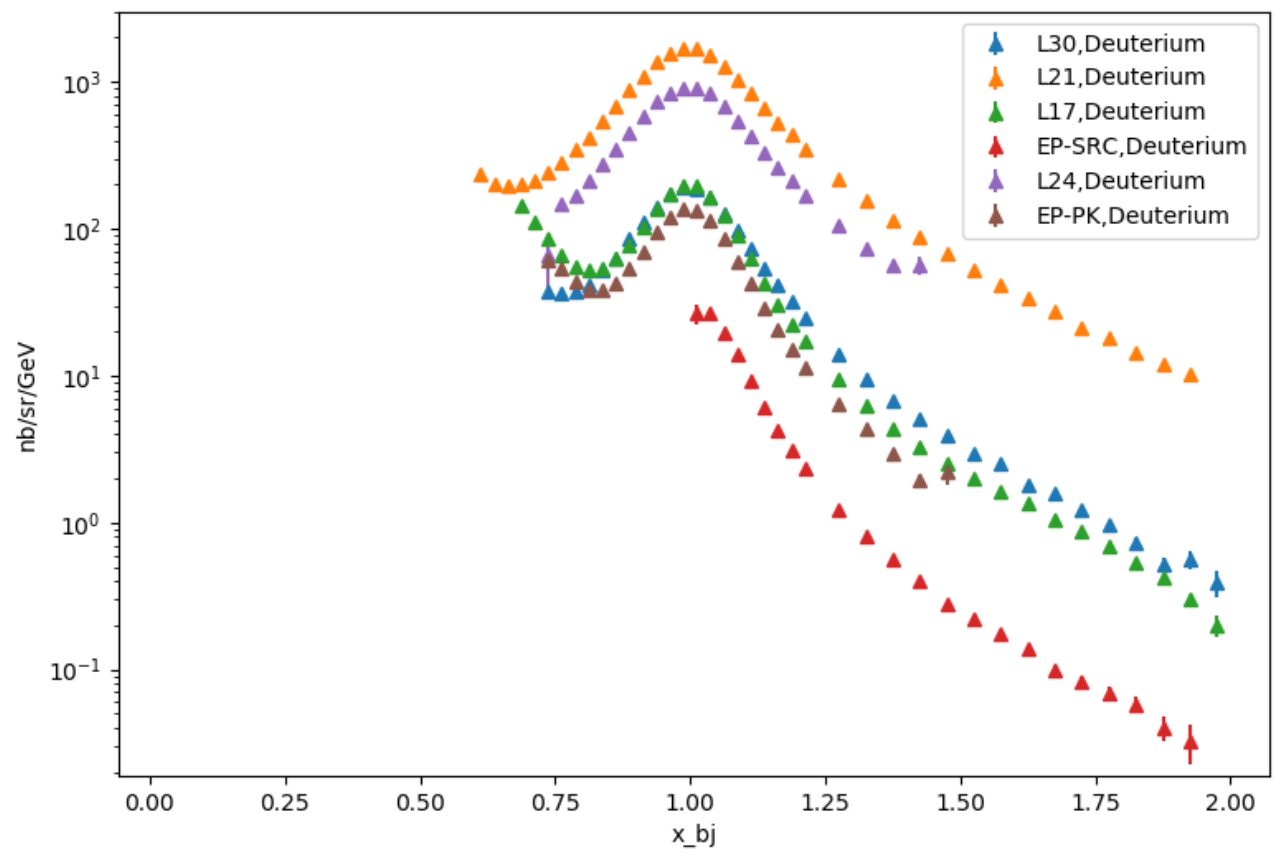

Figure 5-3: Extracted Deuterium nucleus Born cross sections. The error bars represent all point-to-point uncertainties (statistical and systematic) added in quadrature. Comparing to L17 (green), the L30 data (blue) has lower $Q^{2}$ hence smaller inelastic contamination but larger $\mathrm{QE}$ contribution. The combination of those two effects happen to create similar total cross sections for L17 and L30 at the $x_{b j}=1 \mathrm{QE}$ peak. 


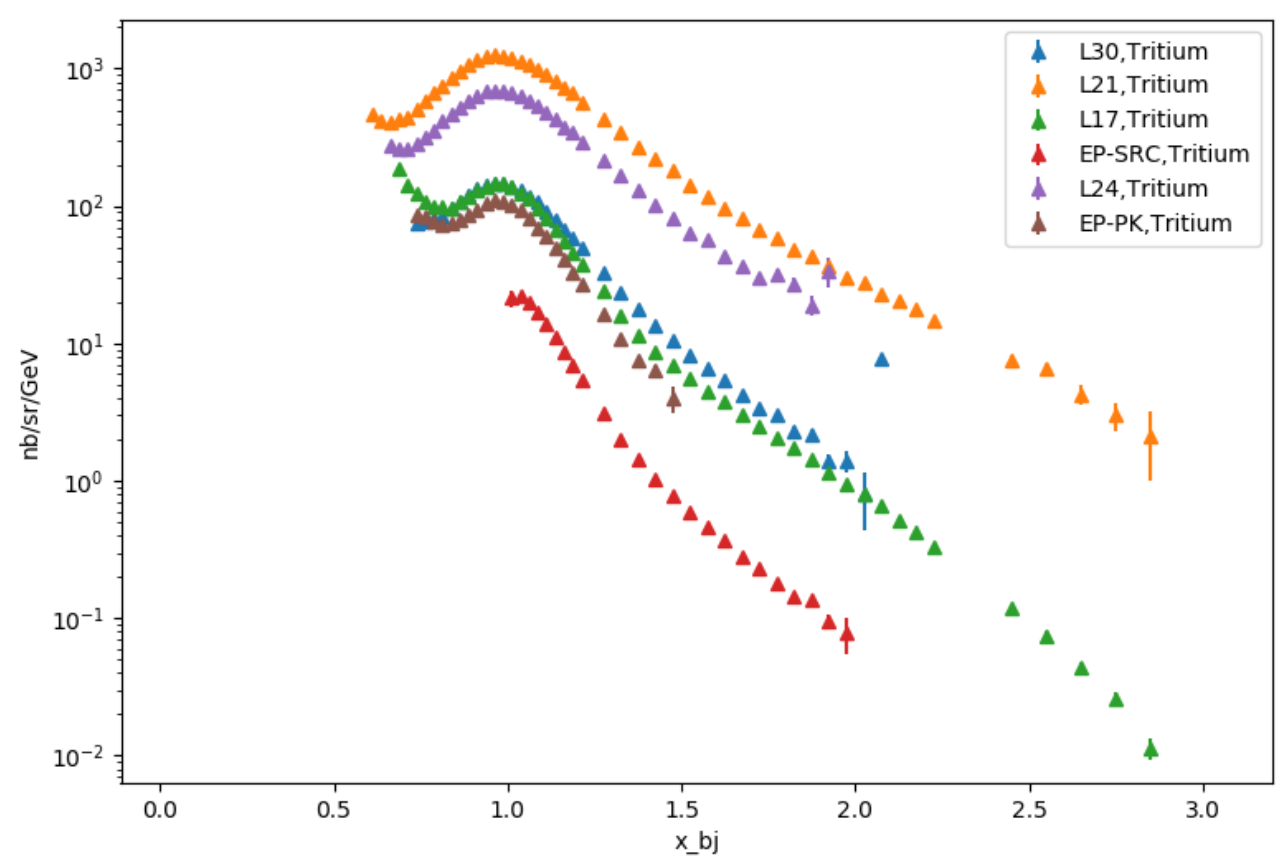

Figure 5-4: Extracted Tritium Born cross sections from various kinematics.

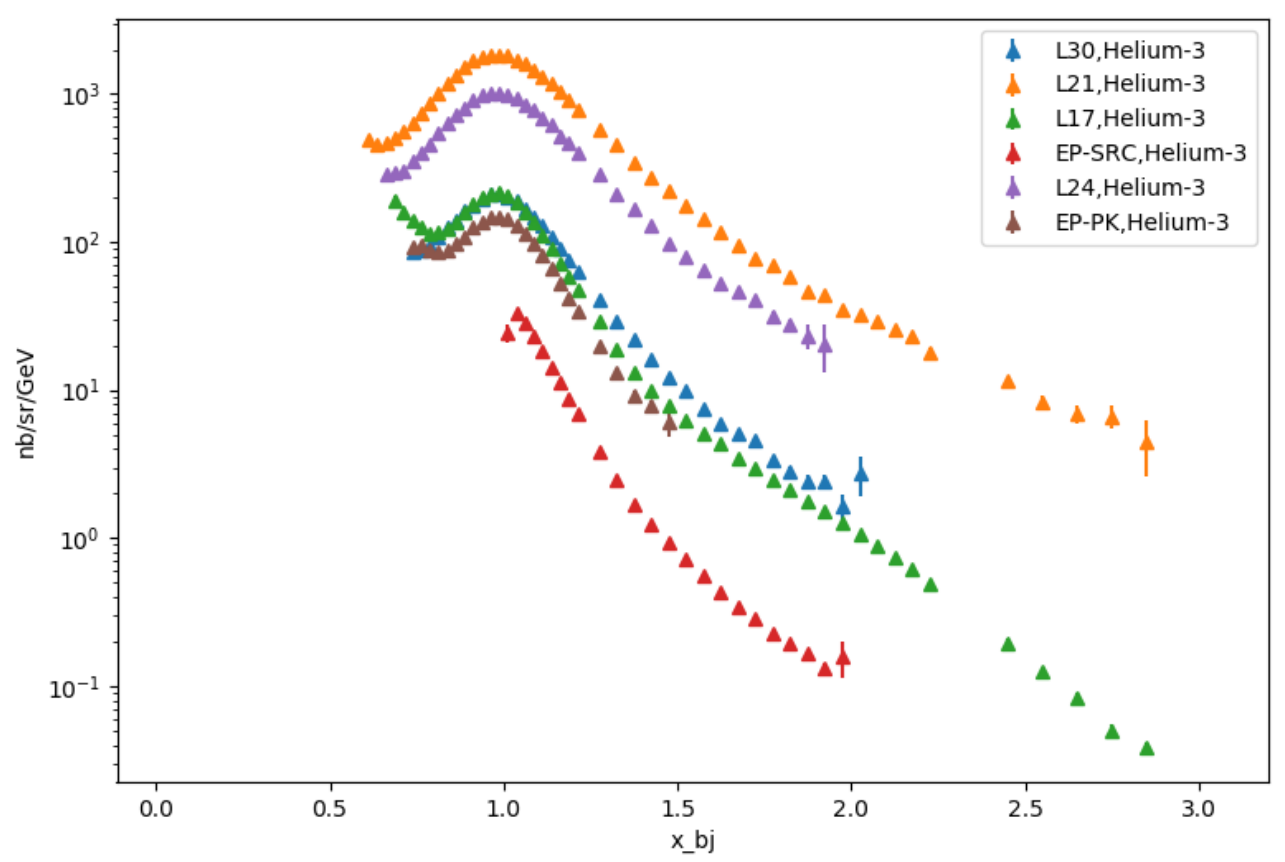

Figure 5-5: Extracted Helium-3 Born cross sections from various kinematics. 


\subsection{1 $\quad F(y)$ Fitting}

With Eq. 4.6, $F(y)$ is extracted from the measured cross section:

$$
F(y)=\frac{\sigma^{\text {data }}-\sigma_{Q E}^{X E M C}}{\left(Z \widetilde{\sigma}_{p}+N \widetilde{\sigma}_{n}\right)} \frac{q}{\sqrt{M^{2}+(y+q)^{2}}}
$$

The extracted tritium, helium-3, and deuterium $F(y)$ from five angles are shown in Fig. 5-6 to Fig. 5-8, Results from L17 and EP are in good agreement since their $Q^{2}$ is large enough to reach the $y$ scaling region. New $F(y)$ fittings are performed with these two data sets. The obtained new parameters are listed in Table 5.1. Data to simulation comparisons are repeated with those new parameters. The extracted cross sections are consistent with the first iteration.

\begin{tabular}{llllll}
\hline & f0 & B & alpha & a & b \\
\hline Deuterium & $9.071 \mathrm{E}-03$ & $4.681 \mathrm{E}-04$ & $4.256 \mathrm{E}+01$ & $8.433 \mathrm{E}-03$ & $7.198 \mathrm{E}-03$ \\
\hline Tritium & $4.873 \mathrm{E}-03$ & $3.017 \mathrm{E}-03$ & $9.611 \mathrm{E}+01$ & $2.852 \mathrm{E}-03$ & $1.424 \mathrm{E}-02$ \\
\hline Helium-3 & $5.144 \mathrm{E}-03$ & $2.741 \mathrm{E}-03$ & $6.953 \mathrm{E}+01$ & $2.528 \mathrm{E}-03$ & $1.374 \mathrm{E}-02$ \\
\hline
\end{tabular}

Table 5.1: $F(y)$ fitting results from extracted cross sections at L17 and EP. 

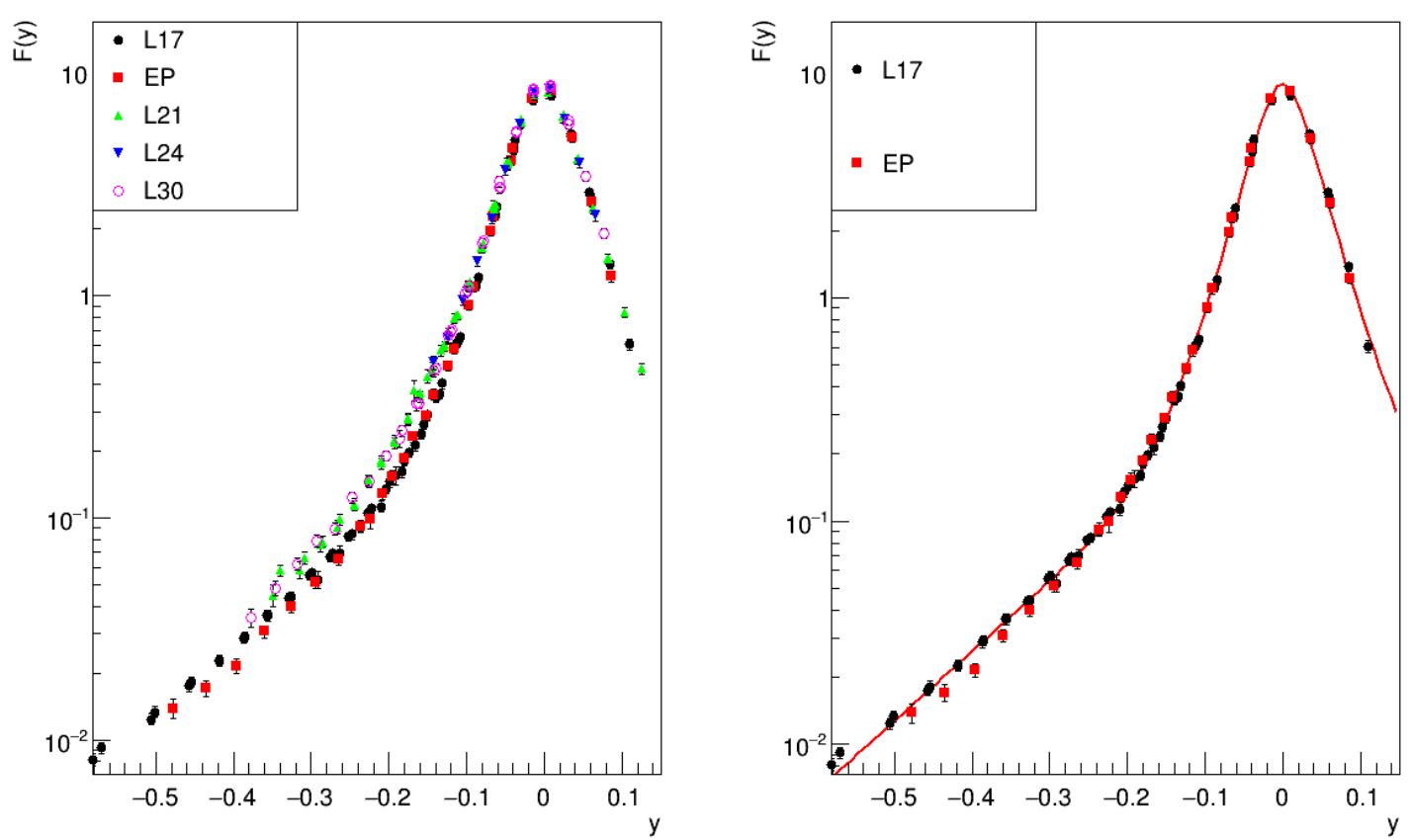

Figure 5-6: Left: extracted deuterium $F(y)$ from this experiment. Right: the high $Q^{2}$ data (L17 and EP) are used for new $F(y)$ fitting.
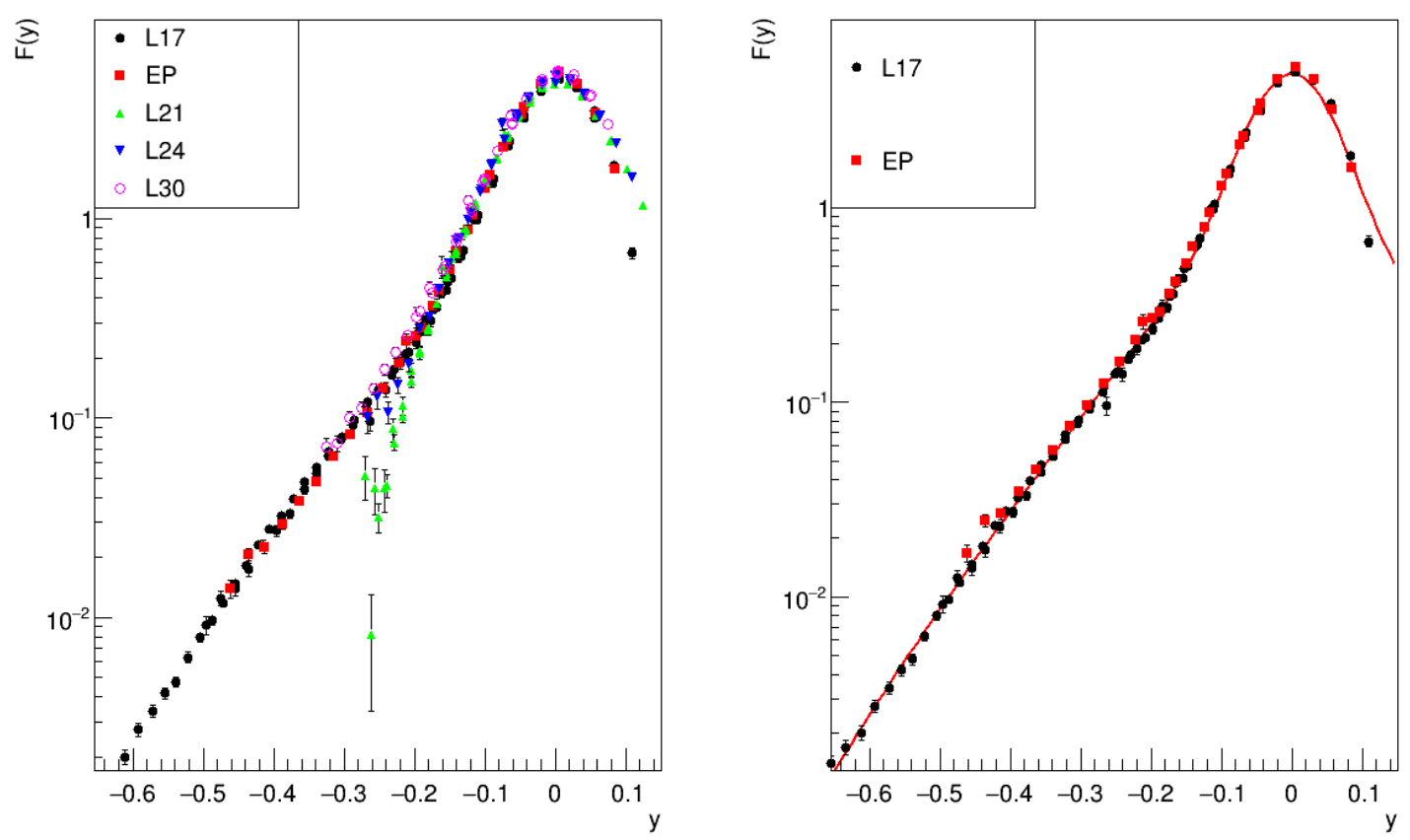

Figure 5-7: Left: extracted tritium $F(y)$ from this experiment. Right: the high $Q^{2}$ data (L17 and EP) are used for new $F(y)$ fitting. 

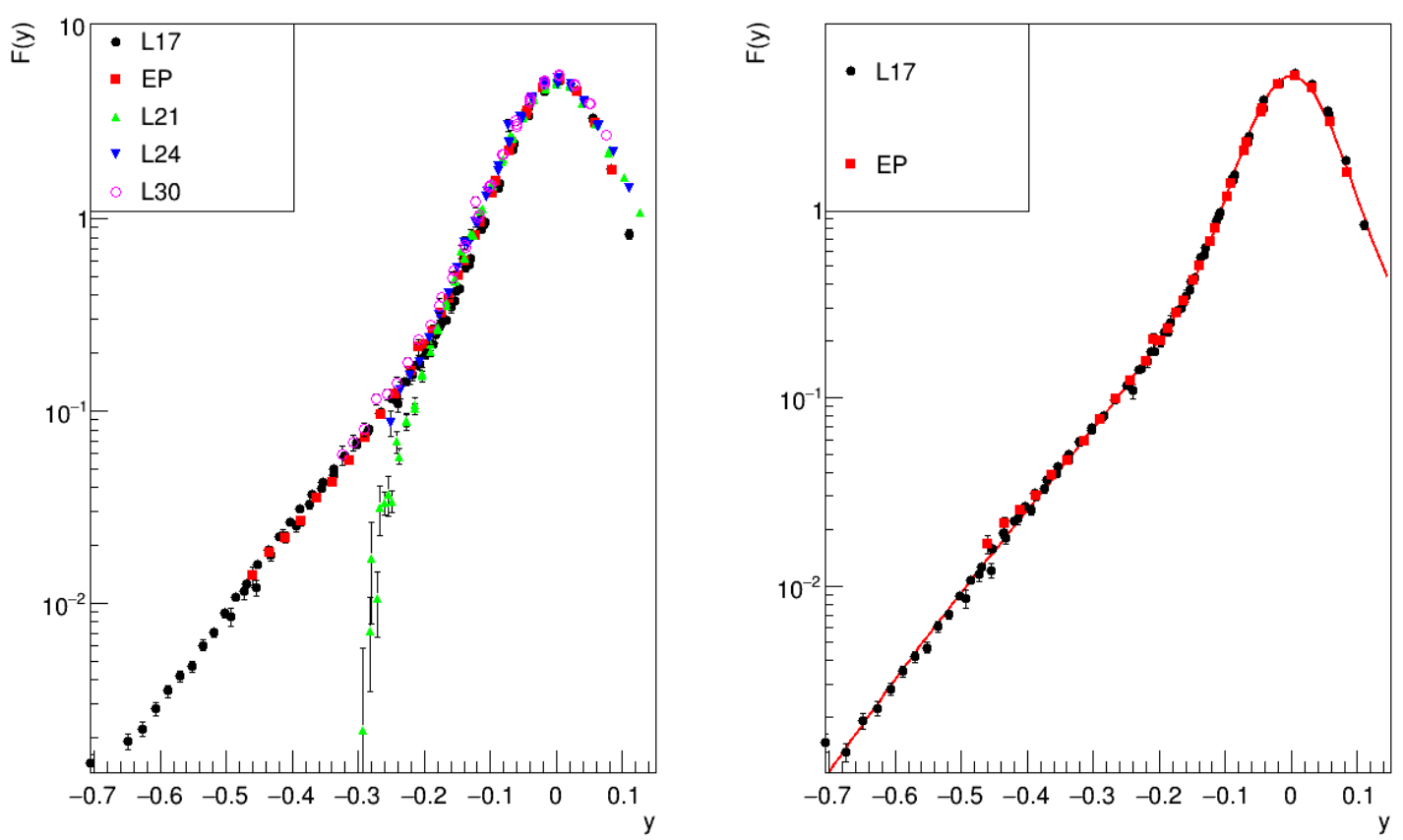

Figure 5-8: Left: extracted helium-3 $F(y)$ from this experiment. Right: the high $Q^{2}$ data (L17 and EP) are used for new $F(y)$ fitting.

\subsection{Cross Section Ratios}

\subsubsection{NN-SRC Plateaus}

${ }^{3} \mathrm{H} /{ }^{2} \mathrm{H}$ and ${ }^{3} \mathrm{He} /{ }^{2} \mathrm{H}$ per-nucleon cross section ratios are extracted with the yield ratio method. Note that the deuterium elastic peak at $x_{b j}=2$ is within the L21 and L17 acceptance. For example, Fig. 5-9 shows that the yield from L21 has a clear peak at $x_{b j}=2$. While the radiative effect from this elastic tail has been taken care of by the XEMC model, the elastic contribution would contaminate the deuterium QE cross section near $x_{b j}=2$. This contamination is worst at L21 which has the lowest $Q^{2}$. To estimate this effect, we fit the QE tail with a Landau function, and the elastic peak with an mirror-inverted Laudau function. From the plot we can see that the fitted elastic peak falls off rapidly, and becomes less than $2 \%$ of the total yield at $x_{b j}=1.8$. In this analysis we cut off the cross section 
ratios at $x_{b j}=1.8$ to stay away from the deuterium elastic contamination. A more careful study with simulation can be carried in the future if necessary.

The ${ }^{3} H /{ }^{2} H$ and ${ }^{3} H e /{ }^{2} H$ per-nucleon cross section ratios at $0.8<x_{b j}<1.8$ from various $Q^{2}$

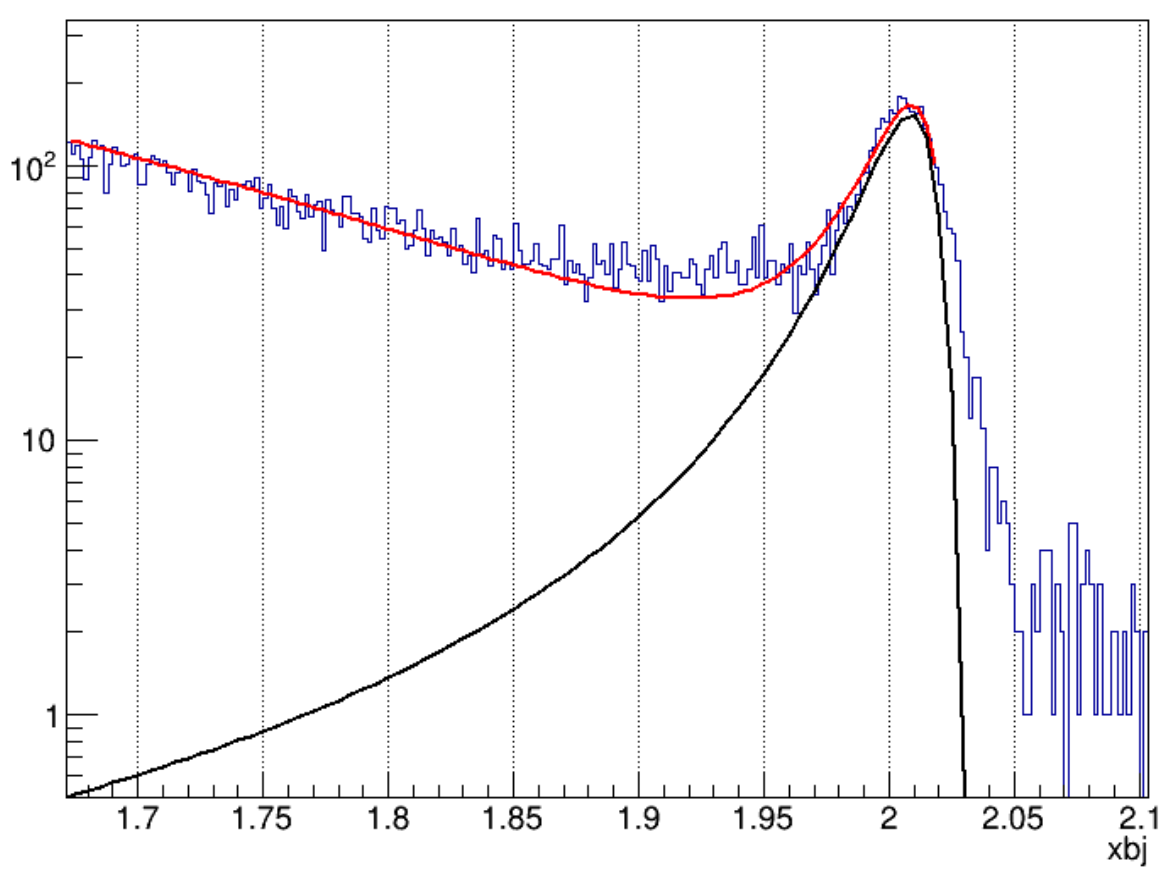

Figure 5-9: Deuterium yield (not normalized) from L21-SRC in log scale. The bump at $x_{b j}=2$ corresponds to deuterium elastic scattering. The red curve is the sum of a Landau distribution and a mirror-inverted Landau distribution. The blue curve is the fitted elastic peak.

are plotted in Fig. 5-10. It shows the transition from no scaling behavior to NN SRC plateau as the $Q^{2}$ increases. This is consistent with the nucleon initial momentum calculation (see Fig. 1-15), which shows that the high momentum nucleons become dominant in the inclusive QE scattering as $Q^{2}$ increases.

The height of ${ }^{3} \mathrm{H} /{ }^{2} \mathrm{H}$ and ${ }^{3} \mathrm{He} /{ }^{2} \mathrm{H}$ NN SRC plateaus are fitted with high $Q^{2}$ data (L17 and $\mathrm{EP})$ to get $a_{2}^{*}(A=3)$. In this process, statistical and point-to-point systematic uncertainties 

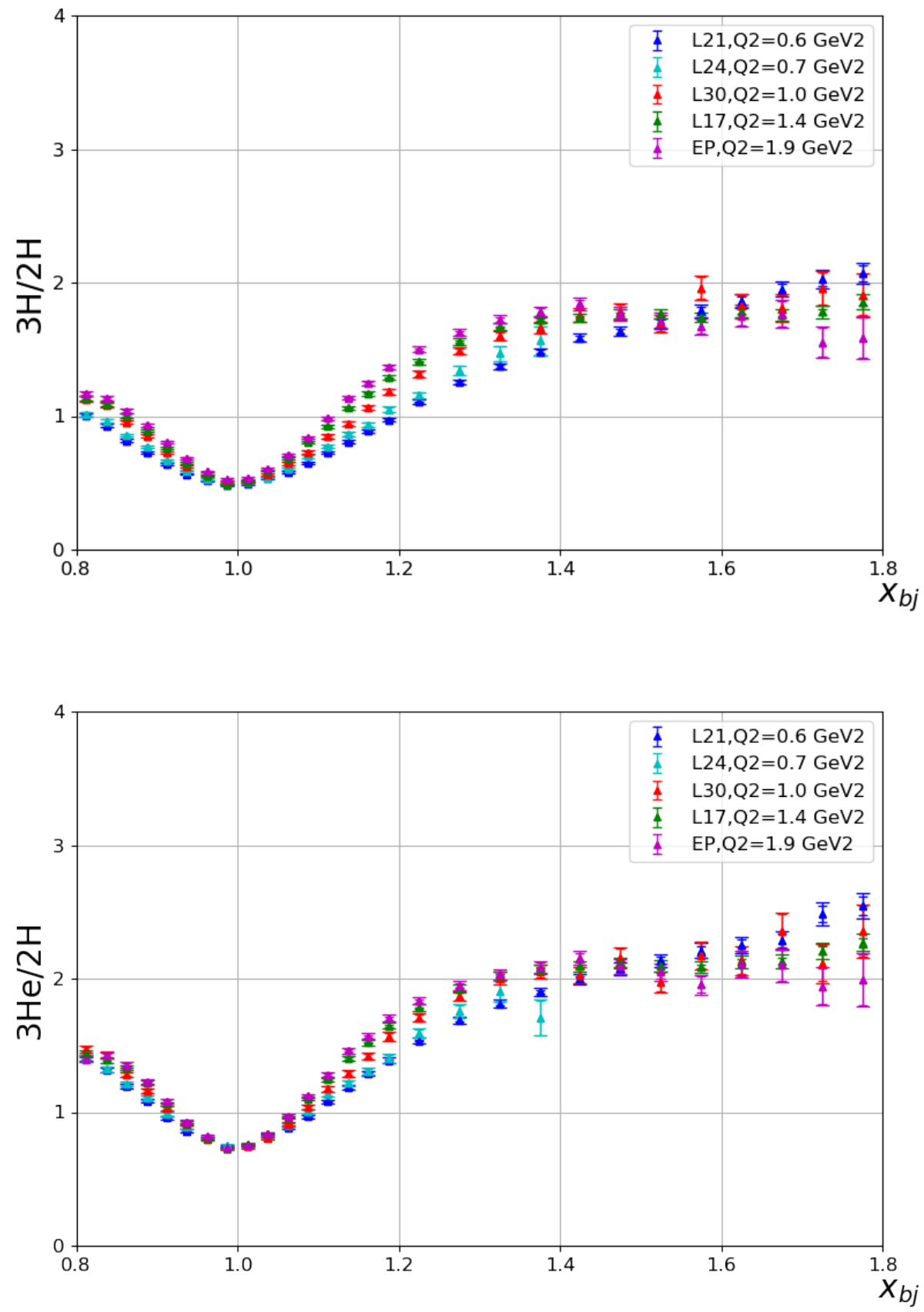

Figure 5-10: Tritium to deuterium (top), and helium-3 to deuterium (bottom) cross section ratios with respect to $x_{b j}$ from various kinematics settings. $Q^{2}$ is quoted at the QE peak. The double error bars represent the statistical uncertainties and the sum of statistical and systematic point-to-point uncertainties. 


\begin{tabular}{lcc}
\hline Observables & $x_{b j}$ range & Ratio \\
\hline $3 \mathrm{He} / 2 \mathrm{H}$ & $1.35-1.70$ & $2.0943 \pm 0.0097$ \\
\hline $3 \mathrm{He} / 2 \mathrm{H}$ & $1.40-1.70$ & $2.0994 \pm 0.0116$ \\
\hline $3 \mathrm{He} / 2 \mathrm{H}$ & $1.40-1.75$ & $2.1040 \pm 0.0127$ \\
\hline $3 \mathrm{H} / 2 \mathrm{H}$ & $1.35-1.70$ & $1.7544 \pm 0.0087$ \\
\hline $3 \mathrm{H} / 2 \mathrm{H}$ & $1.40-1.70$ & $1.7576 \pm 0.0096$ \\
\hline $3 \mathrm{H} / 2 \mathrm{H}$ & $1.40-1.75$ & $1.7576 \pm 0.0098$ \\
\hline $3 \mathrm{H} / 3 \mathrm{He}$ & $1.35-1.70$ & $0.8392 \pm 0.0026$ \\
\hline $3 \mathrm{H} / 3 \mathrm{He}$ & $1.40-1.70$ & $0.8391 \pm 0.0028$ \\
\hline $3 \mathrm{H} / 3 \mathrm{He}$ & $1.40-1.75$ & $0.8369 \pm 0.0033$ \\
\hline
\end{tabular}

Table 5.2: The heights of tritium (helium-3) to deuterium, as well as tritium to helium-3 plateaus fitted with L17 and EP data. Fits were performed with different ranges of $x_{b j}$ for the stability check. Fluctuations of the result are included in the fitting uncertainties.

are added in quadrature. Additional corrections are required to remove the central motion of SRC pairs to extract the actual $a_{2}^{*}(A=3) .1 .4<x_{b j}<1.7$ is the conventional fitting range from previous experiments [72] [25]. This is also the range with flat ratios from our experiment results. We varied the fitting range to estimate the uncertainty. Details are listed in Table 5.2. From the fits we got $a_{2}\left({ }^{3} \mathrm{He}\right)=2.10 \pm 0.01$ and $a_{2}\left({ }^{3} H\right)=1.76 \pm 0.01$. The ${ }^{3} \mathrm{He} /{ }^{2} \mathrm{H}$ NN SRC ratio is consistent with the result from the E02-019 experiment [20]. We introduced the light cone variable [16]

$$
\alpha_{2 N}=2-\frac{q_{-}+2 m_{N}}{2 m_{N}}\left(1+\frac{\sqrt{W_{2 N}^{2}-4 m_{N}^{2}}}{W_{2 N}}\right)
$$

as an alternative scaling variable instead of $x_{b j}$. Here $m_{N}$ is the nucleon mass, $q_{-}=q_{0}-|\mathbf{q}|$, $W_{2 N}^{2}=\left(q+2 m_{N}\right)^{2}=-Q^{2}+4 q_{o} m_{N}+4 m_{N}^{2}$ with $Q^{2}=\mathbf{q}^{2}-q_{0}^{2} \cdot \alpha_{2 N}$ takes into account the recoil energy and momentum carried by the spectator nucleon in the NN SRC to better identify 2N SRCs. In the $Q^{2} \rightarrow \infty$ limit, $\alpha_{2 N} \rightarrow x_{b j}$. Fig. 5-12 showed that the $\alpha_{2 N}$ scaling removed the $Q^{2}$ dependence in the transition region between the QE tails and the $2 \mathrm{~N}-\mathrm{SRC}$ plateaus $\left(1<x_{b j}<1.4\right)$. 

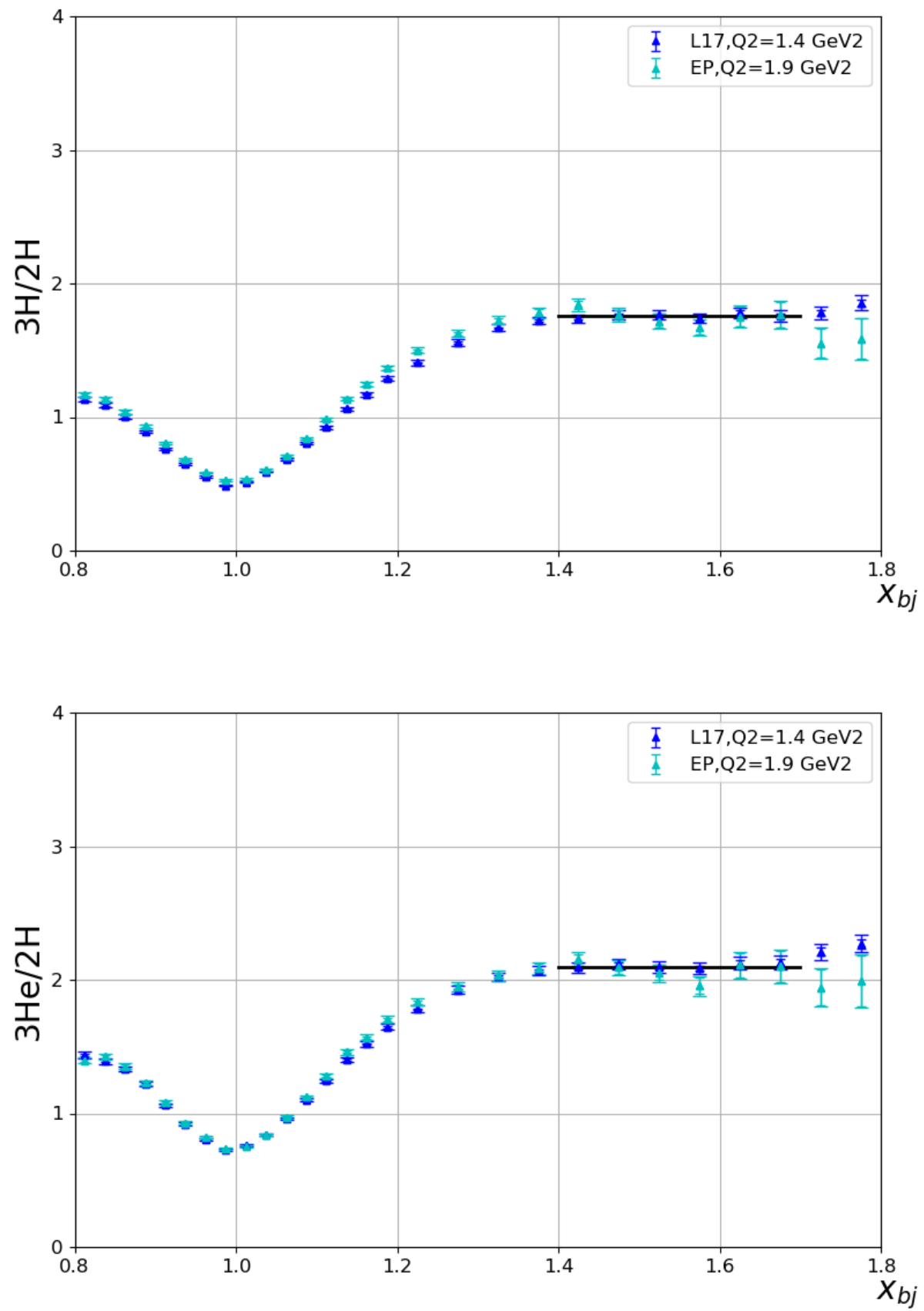

Figure 5-11: Fit the 2N-SRC plateaus with tritium to deuterium (top), and helium-3 to deuterium (bottom) cross section ratios from L17 and EP. 

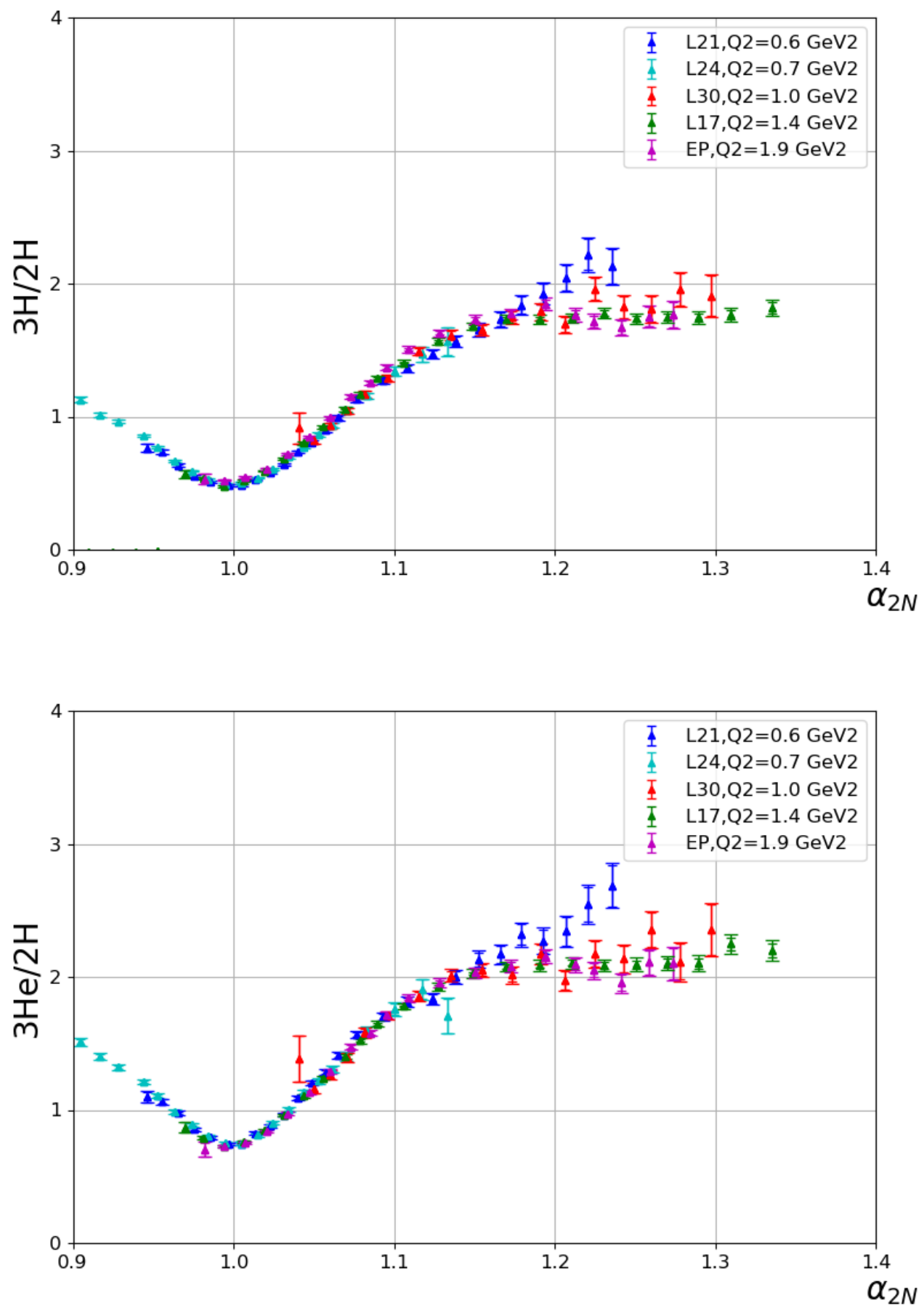

Figure 5-12: Tritium to deuterium (top), and helium-3 to deuterium (bottom) cross section ratios with respect to $\alpha_{2 N}$. 


\subsubsection{The Isospin Preference in $\mathrm{A}=3$ Nuclei}

The tritium to helium-3 cross section ratios are extracted at $0.7<x_{b j}<3$ from various kinematics(see Fig. 5-13). At $x_{b} j<2$ its shape is very similar to the nucleus-to-deuterium ratio: a dip at $x_{b j}=1$ that indicates a small difference of nucleon momentum smearing between tritium and helium-3, then a relative flat region between $1.4<x_{b j}<1.7$. The height of ${ }^{3} \mathrm{H} /{ }^{3} \mathrm{He}$ ratio between $1.4<x_{b j}<1.7$ is fitted to be $0.84 \pm 0.01$. This value deviates from both the $n p$-dominance limit where

$$
\frac{\sigma_{3} H}{\sigma_{3} H e}=\frac{\sigma_{n p}+\sigma_{n}}{\sigma_{n p}+\sigma_{p}} \simeq \frac{\sigma_{n p}}{\sigma_{n p}}=1
$$

and the no-isospin preference assumption that

$$
\frac{\sigma_{3} H}{\sigma_{3} H e}=\frac{2 \sigma_{n}+\sigma_{p}}{\sigma_{n}+2 \sigma_{p}} \stackrel{\sigma_{p} \sim 3 \sigma_{n}}{\longrightarrow} 0.7
$$

- The $n p / p p$ SRC pair ratio in $A=3$ Nuclei can be extracted from the height of plateau with a toy model of pair-counting. For a nucleus with $Z$ protons and $N$ neutrons, assuming that any two nucleons in that nucleus are equally likely to move close to each other, then the chance of forming any two-nucleon pairs are $f(A)=A(A-1) / 2$ among which $N Z$ are $n p$ pairs, and $N(N-1) / 2, Z(Z-1) / 2$ are $n n$ and $p p$ pairs respectively. If a fraction of $p_{0}$ $n p$ pairs and $p_{1} p p(n n)$ pairs are short-range correlated (in other words, have momentum above thte Fermi level), the inclusive cross section contribution from NN SRC pairs can be approximated by: 


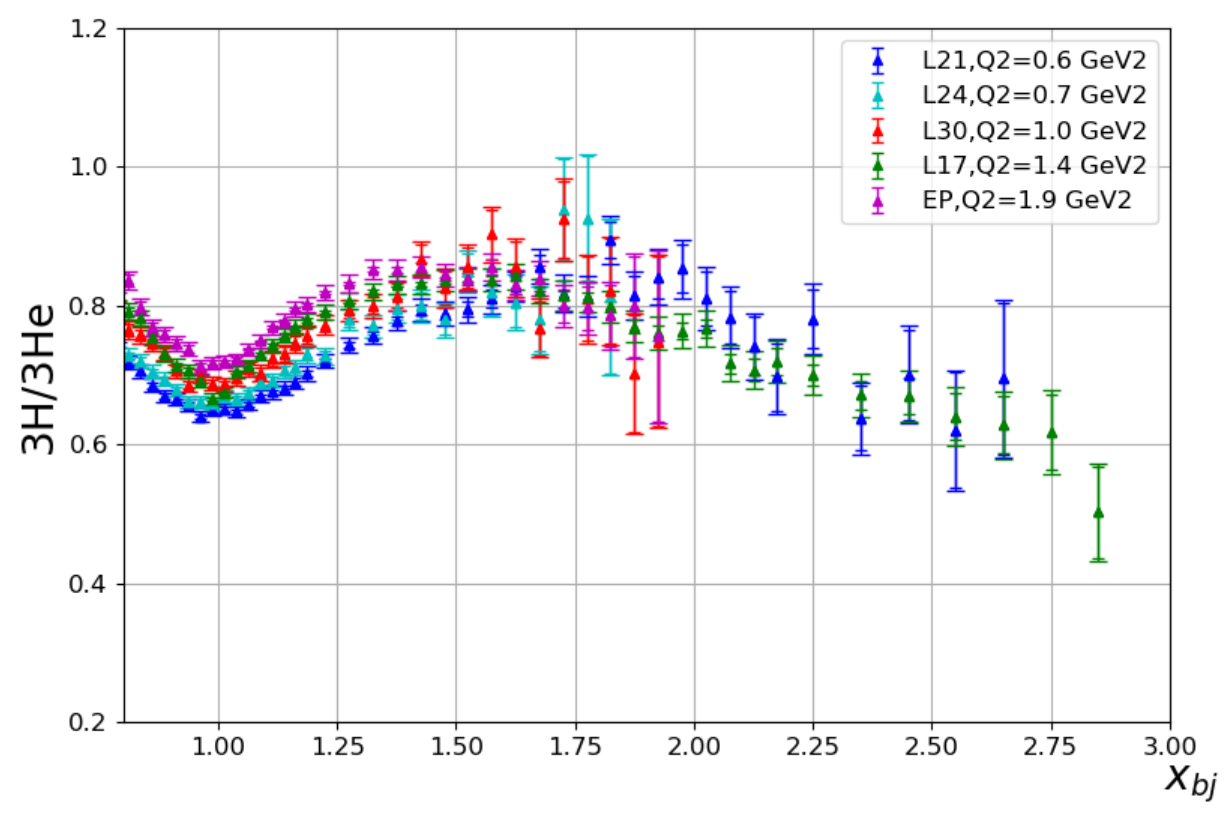

Figure 5-13: Tritium to helium-3 cross section ratio with respect to $x_{b j}$ from various kinematics setting.

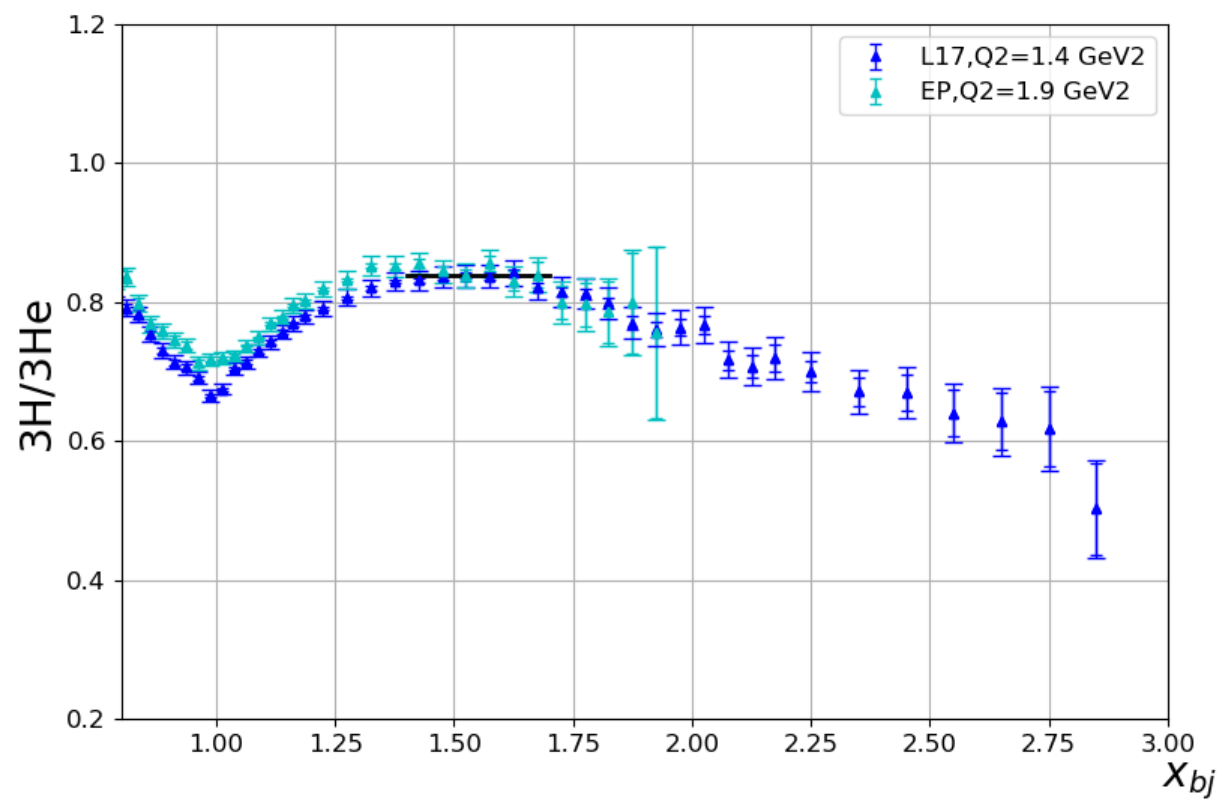

Figure 5-14: Fit the 2N-SRC plateaus with helium-3 to tritium cross section ratios from L17 and EP. 


$$
\sigma_{2 N-S R C}=\frac{1}{f(A)}\left[N Z p_{0}\left(\sigma_{p}+\sigma_{n}\right)+N(N-1) p_{1} \sigma_{n}+Z(Z-1) p_{1} \sigma_{p}\right]
$$

where $\sigma_{p}$ and $\sigma_{n}$ are the on-shell $e p$ and $e n$ elastic cross sections at corresponding kinematics. Here we assume $p p$ and $n n$ pairs have the same chance to form SRC pairs since they are from the same isospin configuration. Assume that tritium and helium-3 share the same $p_{0}$ and $p_{1}$, we can write the tritium to helium-3 inclusive cross section ratio at NN SRC region as

$$
\frac{\sigma\left({ }^{3} H\right)}{\sigma\left({ }^{3} H e\right)}=\frac{2 p_{0}\left(\sigma_{p}+\sigma_{n}\right)+2 p_{1} \sigma_{n}}{2 p_{0}\left(\sigma_{p}+\sigma_{n}\right)+2 p_{1} \sigma_{p}}
$$

The $e p$ and $e n$ elastic cross section ratio is $\sigma_{e p} / \sigma_{e n}=2.7 \pm 0.1$ at L17 and EP kinematics [73]. Substituting this ratio as well as $\sigma\left({ }^{3} H\right) / \sigma\left({ }^{3} \mathrm{He}\right)=0.84 \pm 0.01$ into the above equation gives the $n p$ to $p p$ SRC pair ratio $p_{0} / p_{1}=2.2 \pm 0.2$.

Similarly, the exclusive $\left(e, e^{\prime} p\right)$ cross section from NN SRC is $\sim N Z p_{0}+Z(Z-1) p_{1}$ up to a factor of $\sigma_{p}$. So the exclusive tritium to helium-3 ratio at NN SRC region can be written as:

$$
\frac{\sigma_{e p}\left({ }^{3} H\right)}{\sigma_{e p}\left({ }^{3} H e\right)}=\frac{2 p_{0}}{2 p_{0}+2 p_{1}}
$$

As shown in Fig. 5-15, the $p_{0} / p_{1}$ extracted from the exclusive scattering data from [28] is consistent with what we obtained from this analysis, but has a larger uncertainty.

This toy model separates the SRC pair's cross section contribution into 1) the probability to form a pair from pair counting, 2) the percentage of pairs with high momentum $\left(p_{1}\right.$ and $\left.p_{0}\right)$, 


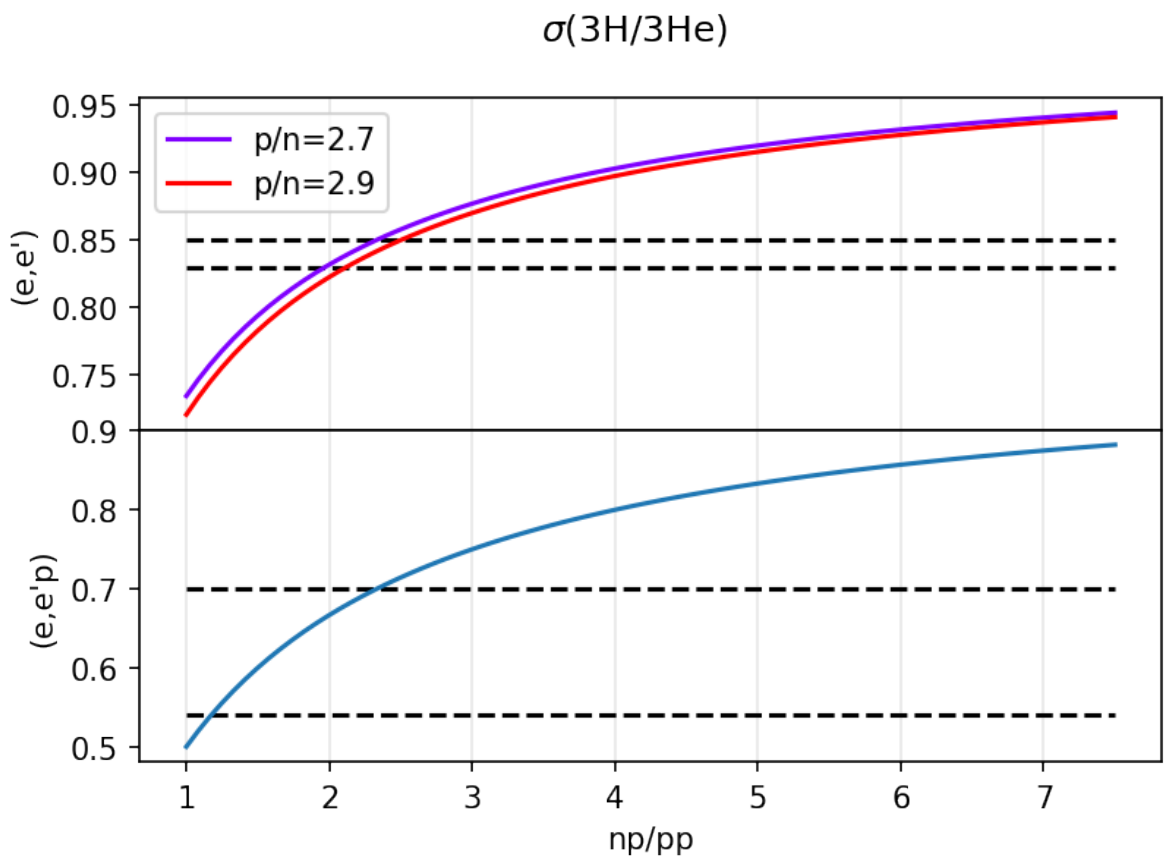

Figure 5-15: $n p$ to $p p$ SRC pair ratio extracted from the inclusive ${ }^{3} H /{ }^{3} H e$ cross section ratio in this thesis (top), and the exclusive $e^{\prime} p$ experiment [28] (bottom).

and 3) the electron-nucleon elastic scattering cross sections. The total cross section ratio of $\sigma 3 \mathrm{H} / \sigma 3 \mathrm{He}$ is used instead of $a_{2}(A=3)$ with the assumption that competing processes are either suppressed by kinematics or cancelled in the ratios, and the nuclear smearing effect is very similar in $A=3$ nuclei. Those assumptions will be carefully re-evaluated in the future analysis. This pair counting approach could be applied on the SRC ratios from other similar nuclei such as ${ }^{48} \mathrm{Ca} /{ }^{40} \mathrm{Ca}[25]$.

Fig. 5-16 shows the nucleon momentum distribution inside tritium as calculated with Av18+UX[74]. Our $n p$ to $p p$ SRC pair ratio of $2.2 \pm 0.2$ is consistent with the calculated $n p / p p \sim 2.4$ at $1.2<q<1.5 \mathrm{fm}^{-} 1$. The same calculation projects the pair momentum distribution into different spin and isospin (ST) configurations to give the $T=0 / T=1$ pair density ratio

$$
\frac{\rho(T 0)}{\rho(T 1)}=\frac{\rho(S T=00)+\rho(S T=10)}{\rho(S T=01)+\rho(S T=11)} \approx 1.23
$$



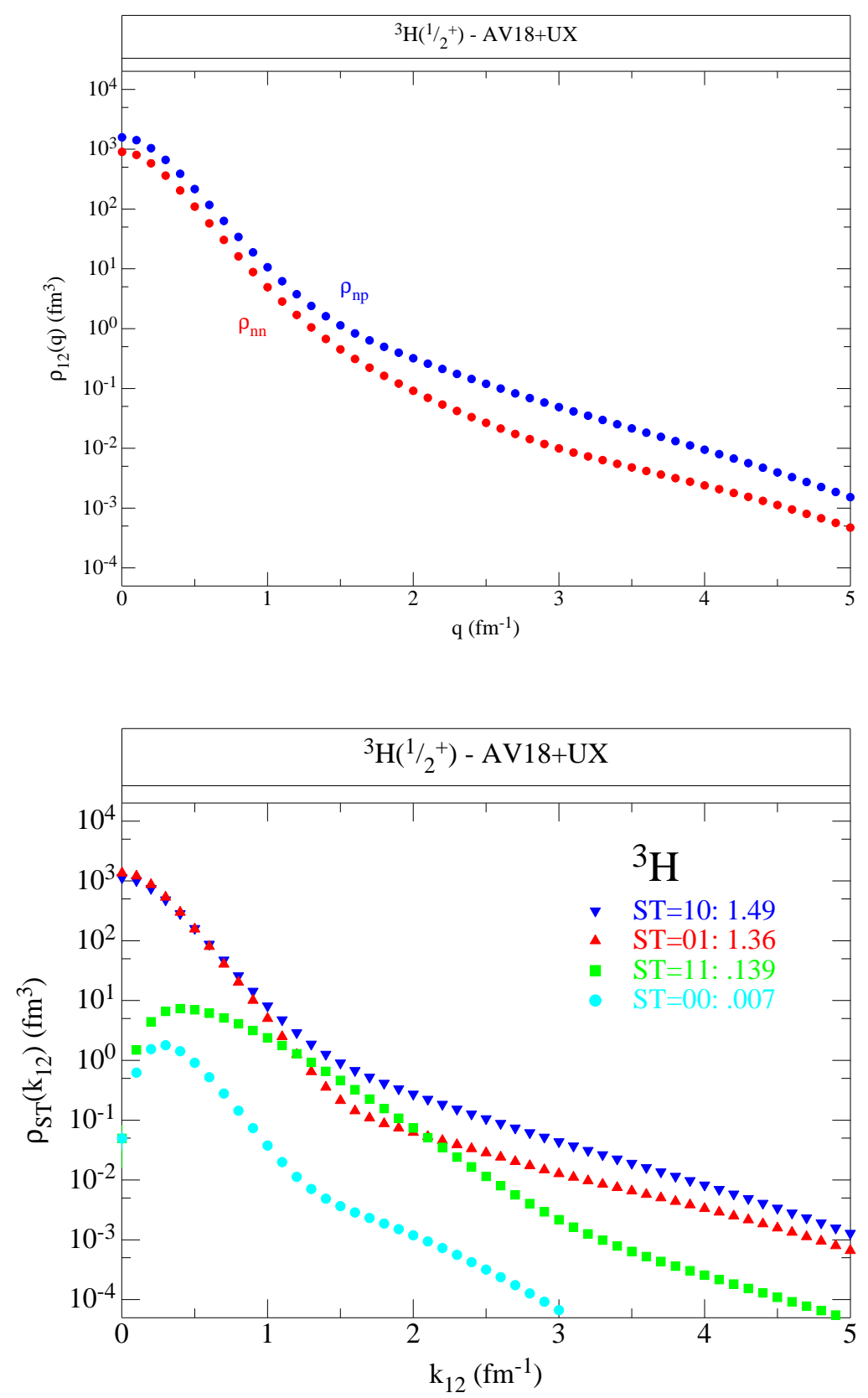

Figure 5-16: The nucleon momentum distribution between nucleon-nucleon pairs as a function of the pair's relative momentum $q=k_{12}=(k 1-k 2) / 2$. The distribution is projected into $n n$ and $n p$ pairs (left) and spin-isospin (ST) configurations (right). The figure on the right also gives the density integral of each configuration. Figures taken from [74]. 
at the Fermi level $\left(k_{12} \approx 1.3 \mathrm{fm}^{-1}\right.$. That is a $20 \%$ enhancement of isospin $T=0$ state in the $A=3$ SRC pairs. If we simply apply the known NN isospin configuration, which is, equal number of isospin $T=1 n n, n p$, and $p p$ pairs plus $n p$ pairs from isospin $T=0$ on the extracted $n p / p p$ SRC pair ratio from this analysis, we get the ratio of $T=0$ to $T=1$ SRC pairs is $\left(p_{0}-p_{1}\right) / p_{1}=1.2 \pm 0.2$. While this number is very close to the calculation, this is just a rough estimation of inclusive cross section ratio as the contribution from the lowest momentum nucleons. We are working with several groups of theorists to understand the cross section ratio results and its implication on the isospin dependence of NN SRC through QMC and $\chi \mathrm{EFT}$ calculations.

\subsubsection{Beyond 2N SRCs}

Two kinematic settings, L21 at $Q^{2}=0.6 \mathrm{GeV}^{2}$ and L17 at $Q^{2}=1.4 \mathrm{GeV}^{2}$ provide ${ }^{3} H /{ }^{3} H e$ cross section ratios at $2<x_{b j}<3$. As shown in Fig. 5-17, the ratio is around 0.7 with large statistical uncertainties. Due to the lack of statistics we can not make a statement on the 3N SRC plateau. But it's worth mentioning that recent calculations [16] suggests that $Q^{2}>5 \mathrm{GeV}^{2}$ is required to reach the possible $3 \mathrm{~N}$ SRC dominant region at $2<x_{b j}$. 


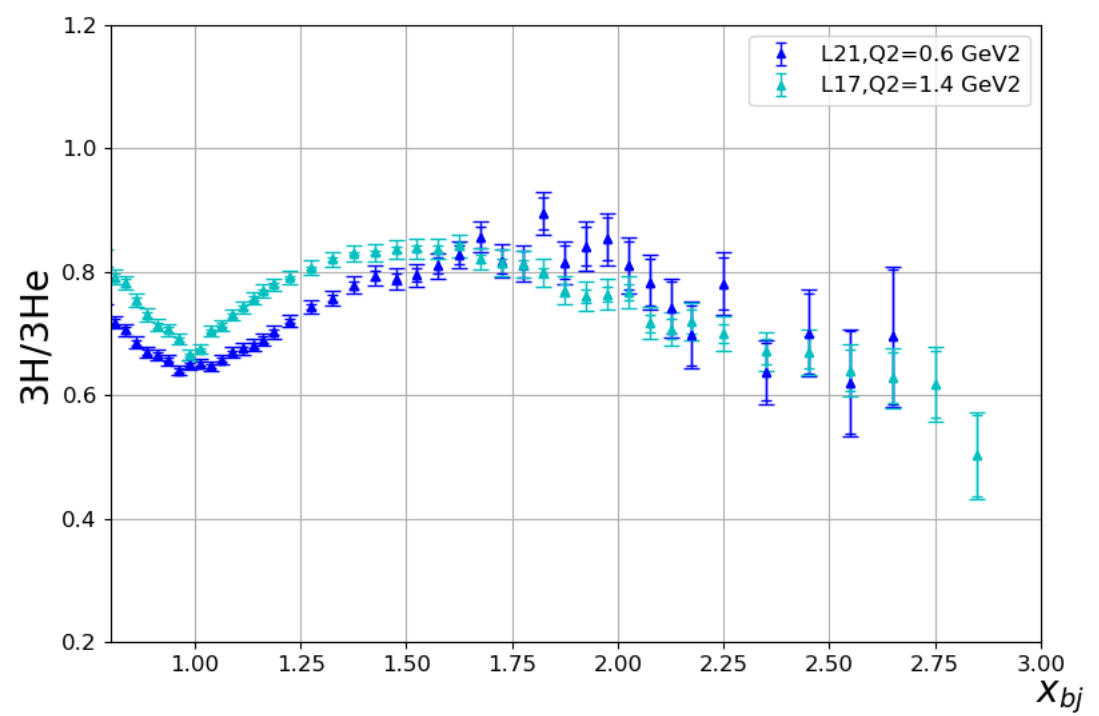

Figure 5-17: Tritium to helium-3 cross section ratio from L17 and L21. 


\section{Chapter 6}

\section{Conclusions}

This thesis presents the deuterium, tritium, and helium-3 inclusive cross sections at $0.6<$ $x_{b j}<3$ and $0.6<Q^{2}<1.9 \mathrm{GeV}^{2}$. The high $Q^{2}$ data shows scaling behavior at $-0.7<$ $y<0$. The tritium and helium-3 F(y) fitting parameters for the XEMC cross section model have been updated with this new measurement. The helium-3 to deuterium cross section ratio at $Q^{2}>1.3 \mathrm{GeV}^{2}$ shows a plateau at the $\mathrm{NN}$ SRC dominant kinematics. The plateau height of $2.10 \pm 0.01$ is consistent with previous measurements [72]. The first result of tritium to helium-3, tritium to deuterium cross section ratios at $x_{b j}>1$ are provided. The cross section ratios show strong $Q^{2}$ dependence at the onset of NN SRC plateaus. The height of plateau are fitted with $Q^{2}>1.4 \mathrm{GeV}^{2}$ data at $1.4<x_{b j}<1.7$. The tritium to helium-3 cross section ratio of $0.84 \pm 0.01$ at $\mathrm{NN}$ SRC dominant region is different from predictions with no isospin preference and with complete isospin singlet dominance. Assuming that nuclear smearing and many other effects are cancelled in the tritium to helium-3 ratio, we extracted the $n p$ to $p p$ SRC pair ratio of $2.2 \pm 0.2$ with a pair-counting model. This may imply a $20 \%$ more $T=0$ pairs in NN SRC comparing to the $T=1$ pairs. The $x_{b j}>2$ tritium to helium-3 ratio is between 0.6 and 0.8 . We cannot make any conclusion on the $3 \mathrm{~N}$ SRC plateau due to the lack of statistics.

This tritium and helium-3 cross sections and ratios can be used to test theory models at 
SRC region. The cross sections at the QE peak can also help determine the tritium nuclear corrections, which is important in the structure function $F_{2}$ extraction from DIS tritium data. On the other hand, we need theory input to extract the actual isospin configuration in $2 \mathrm{~N}$ SRCs from the height of $2 \mathrm{~N}$ plateau, and to understand the ratios at $x_{b j}>2$. There are on-going theory efforts including $\chi \mathrm{EFT}$ and spectral function calculations [75] [76]. We are expecting to see theory to data comparisons in the near future. A separate analysis of the tritium and helium-3 cross sections at QE peak is carrying on to extract the neutron magnetic form factor [77. Our tritium and helium-3 ratio at $2 \mathrm{~N}$ SRC region will be combined with the ratio of two in DIS region from the MARATHON experiment to study the EMC-SRC relation. Cross section tables and other results are available at https://wiki.jlab.org/tegwiki/index.php/Wiki-page_for_E12-11-112. 


\section{Bibliography}

[1] L. FRANkFURT, M. SARGSIAN, and M. STRIKMAN, Int. J. Mod. Phys. A 23, 2991 (2008), arXiv:0806.4412.

[2] C. C. D. Atti and S. Simula, 53, 40 (1995), arXiv:9507024 [nucl-th].

[3] L. Lapikás, Nuclear Physics A 553, 297 (1993).

[4] R. Subedi, R. Shneor, P. Monaghan, B. D. Anderson, K. Aniol, J. Annand, J. Arrington, H. Benaoum, F. Benmokhtar, W. Boeglin, J.-P. Chen, S. Choi, E. Cisbani, B. Craver, S. Frullani, F. Garibaldi, S. Gilad, R. Gilman, O. Glamazdin, J.-O. Hansen, D. W. Higinbotham, T. Holmstrom, H. Ibrahim, R. Igarashi, C. W. de Jager, E. Jans, X. Jiang, L. J. Kaufman, a. Kelleher, a. Kolarkar, G. Kumbartzki, J. J. Lerose, R. Lindgren, N. Liyanage, D. J. Margaziotis, P. Markowitz, S. Marrone, M. Mazouz, D. Meekins, R. Michaels, B. Moffit, C. F. Perdrisat, E. Piasetzky, M. Potokar, V. Punjabi, Y. Qiang, J. Reinhold, G. Ron, G. Rosner, a. Saha, B. Sawatzky, a. Shahinyan, S. Sirca, K. Slifer, P. Solvignon, V. Sulkosky, G. M. Urciuoli, E. Voutier, J. W. Watson, L. B. Weinstein, B. Wojtsekhowski, S. Wood, X.-C. Zheng, and L. Zhu, Science 320, 1476 (2008), arXiv:0908.1514.

[5] S. C. Pieper and R. B. Wiringa, Annu. Rev. Nucl. Part. Sci. 51, 53 (2001).

[6] B. Povh, K. Rith, C. Scholz, and F. Zetsche, Particles and Nuclei: An Introduction to the Physical Concepts (2006) p. 388. 
[7] R. Machleidt, Phys. Rev. C 63, 024001 (2001).

[8] V. G. J. Stoks, R. A. M. Klomp, C. P. F. Terheggen, and J. J. de Swart, Phys. Rev. C 49, 2950 (1994).

[9] R. B. Wiringa, V. G. J. Stoks, and R. Schiavilla, Phys. Rev. C 51, 38 (1995), arXiv:9408016 [nucl-th].

[10] N. Ishii, S. Aoki, and T. Hatsuda, Phys. Rev. Lett. 99, 022001 (2007).

[11] Z. Ye, Short Range Correlations in Nuclei at Large $x_{-} b j$ through Inclusive Quasi-Elastic Electron Scattering, Ph.D. thesis, University of Virgina (2013).

[12] R. Schiavilla, R. B. Wiringa, S. C. Pieper, and J. Carlson, Phys. Rev. Lett. 98, 132501 (2007), arXiv:0611037 [nucl-th].

[13] S. C. Pieper, (2007), 10.1393/ncr/i2009-10039-1, arXiv:0711.1500.

[14] G. B. King, L. Andreoli, S. Pastore, M. Piarulli, R. Schiavilla, R. B. Wiringa, J. Carlson, and S. Gandolfi, (2020), arXiv:2004.05263.

[15] C. C. degli Atti, E. Pace, and G. Salme, Nucl. Phys. A 497, 361 (1989).

[16] D. B. Day, L. L. Frankfurt, M. M. Sargsian, and M. I. Strikman, (2018), arXiv:1803.07629.

[17] L. Weinstein, "Electrons for neutrinos," Neutrino Cross Section Strategy Workshop (2018).

[18] J. Arrington, D. W. Higinbotham, G. Rosner, and M. Sargsian, Prog. Part. Nucl. Phys. 67, 898 (2012), arXiv:1104.1196. 
[19] E. Piasetzky, M. Sargsian, L. Frankfurt, M. Strikman, and J. W. Watson, Phys. Rev. Lett. 97, 13 (2006).

[20] N. Fomin, J. Arrington, R. Asaturyan, F. Benmokhtar, W. Boeglin, P. Bosted, a. Bruell, M. H. S. Bukhari, M. E. Christy, E. Chudakov, B. Clasie, S. H. Connell, M. M. Dalton, a. Daniel, D. B. Day, D. Dutta, R. Ent, L. El Fassi, H. Fenker, B. W. Filippone, K. Garrow, D. Gaskell, C. Hill, R. J. Holt, T. Horn, M. K. Jones, J. Jourdan, N. Kalantarians, C. E. Keppel, D. Kiselev, M. Kotulla, R. Lindgren, a. F. Lung, S. Malace, P. Markowitz, P. McKee, D. G. Meekins, H. Mkrtchyan, T. Navasardyan, G. Niculescu, a. K. Opper, C. Perdrisat, D. H. Potterveld, V. Punjabi, X. Qian, P. E. Reimer, J. Roche, V. M. Rodriguez, O. Rondon, E. Schulte, J. Seely, E. Segbefia, K. Slifer, G. R. Smith, P. Solvignon, V. Tadevosyan, S. Tajima, L. Tang, G. Testa, R. Trojer, V. Tvaskis, W. F. Vulcan, C. Wasko, F. R. Wesselmann, S. a. Wood, J. Wright, and X. Zheng, Phys. Rev. Lett. 108, 1 (2012), arXiv:1107.3583.

[21] L. L. Frankfurt, M. I. Strikman, D. B. Day, and M. Sargsyan, Phys. Rev. C 48, 2451 (1993).

[22] D. B. Day, J. S. McCarthy, Z. E. Meziani, R. Minehart, R. Sealock, S. T. Thornton, J. Jourdan, I. Sick, B. W. Filippone, R. D. McKeown, R. G. Milner, D. H. Potterveld, and Z. Szalata, Phys. Rev. Lett. 59, 427 (1987).

[23] P. Solvignon, J. Arrington, D. Day, and D. W. Higinbotham, "Precision measurement of the isospin dependence in the $2 \mathrm{~N}$ and $3 \mathrm{~N}$ short range correlation region," E12-11-112 proposal (2010).

[24] D. W. Higinbotham and O. Hen, Phys. Rev. Lett. 114, 169201 (2015). 
[25] D. Nguyen, Z. Ye, P. Aguilera, Z. Ahmed, H. Albataineh, K. Allada, B. Anderson, D. Anez, K. Aniol, J. Annand, J. Arrington, T. Averett, H. Baghdasaryan, X. Bai, A. Beck, S. Beck, V. Bellini, F. Benmokhtar, A. Camsonne, C. Chen, J. P. Chen, K. Chirapatpimol, E. Cisbani, M. M. Dalton, A. Daniel, D. Day, W. Deconinck, M. Defurne, D. Flay, N. Fomin, M. Friend, S. Frullani, E. Fuchey, F. Garibaldi, D. Gaskell, S. Gilad, R. Gilman, S. Glamazdin, C. Gu, P. Guèye, C. Hanretty, J. O. Hansen, M. H. Shabestari, D. W. Higinbotham, M. Huang, S. Iqbal, G. Jin, N. Kalantarians, H. Kang, A. K. Her, I. Korover, J. LeRose, J. Leckey, S. Li, R. Lindgren, E. Long, J. Mammei, D. J. Margaziotis, P. Markowitz, D. Meekins, Z. E. Meziani, R. Michaels, M. Mihovilovi \v, N. Muangma, C. M. Camacho, B. E. Norum, Nuruzzaman, K. Pan, S. Phillips, E. Piasetzky, I. Pomerantz, M. Posik, V. Punjabi, X. Qian, Y. Qiang, X. Qiu, P. E. Reimer, A. Rakhman, S. Riordan, G. Ron, O. RondonAramayo, A. Saha, L. Selvy, A. Shahinyan, R. Shneor, S. \v, K. Slifer, P. Solvignon, N. Sparveris, R. Subedi, V. Sulkosky, D. Wang, J. W. Watson, L. B. Weinstein, B. Wojtsekhowski, S. A. Wood, I. Yaron, X. Zhan, J. Zhang, Y. W. Zhang, B. Zhao, X. Zheng, P. Zhu, and R. Zielinski, (2020), arXiv:2004.11448.

[26] D. W. Higinbotham, J. Gomez, and E. Piasetzky, (2010), arXiv:1003.4497.

[27] G. Petratos, M. Katramatou, J. Arrington, D. Meekins, and R. Ransome, "MeAsurement of the F2n /F2p, d/u RAtios and A=3 EMC Effect in Deep Inelastic Electron Scattering Off the Tritium and Helium MirrOr Nuclei," .

[28] R. Cruz-Torres, S. Li, F. Hauenstein, A. Schmidt, D. Nguyen, D. Abrams, H. Albataineh, S. Alsalmi, D. Androic, K. Aniol, W. Armstrong, J. Arrington, H. Atac, T. Averett, C. Ayerbe Gayoso, X. Bai, J. Bane, S. Barcus, A. Beck, V. Bellini, H. Bhatt, 
D. Bhetuwal, D. Biswas, D. Blyth, W. Boeglin, D. Bulumulla, A. Camsonne, J. Castellanos, J.-P. Chen, E. Cohen, S. Covrig, K. Craycraft, B. Dongwi, M. Duer, B. Duran, D. Dutta, E. Fuchey, C. Gal, T. Gautam, S. Gilad, K. Gnanvo, T. Gogami, J. Gomez, C. Gu, A. Habarakada, T. Hague, O. Hansen, M. Hattawy, O. Hen, D. Higinbotham, E. Hughes, C. Hyde, H. Ibrahim, S. Jian, S. Joosten, A. Karki, B. Karki, A. Katramatou, C. Keppel, M. Khachatryan, V. Khachatryan, A. Khanal, D. King, P. King, I. Korover, T. Kutz, N. Lashley-Colthirst, G. Laskaris, W. Li, H. Liu, N. Liyanage, D. Lonardoni, R. Machleidt, L. Marcucci, P. Markowitz, R. McClellan, D. Meekins, S. Mey-Tal Beck, Z.-E. Meziani, R. Michaels, M. Mihovilovič, V. Nelyubin, N. Nuruzzaman, M. Nycz, R. Obrecht, M. Olson, L. Ou, V. Owen, B. Pandey, V. Pandey, A. Papadopoulou, S. Park, M. Patsyuk, S. Paul, G. Petratos, E. Piasetzky, R. Pomatsalyuk, S. Premathilake, A. Puckett, V. Punjabi, R. Ransome, M. Rashad, P. Reimer, S. Riordan, J. Roche, F. Sammarruca, N. Santiesteban, B. Sawatzky, E. Segarra, B. Schmookler, A. Shahinyan, S. Širca, N. Sparveris, T. Su, R. Suleiman, H. SzumilaVance, A. Tadepalli, L. Tang, W. Tireman, F. Tortorici, G. Urciuoli, M. Viviani, L. Weinstein, B. Wojtsekhowski, S. Wood, Z. Ye, Z. Ye, and J. Zhang, Phys. Lett. B 797, $134890(2019)$

[29] O. Hen, E. Piasetzky, and L. B. Weinstein, Phys. Rev. C - Nucl. Phys. 85 (2012), 10.1103/PhysRevC.85.047301, arXiv:1202.3452.

[30] R. Cruz-Torres, Two-Nucleon Short-Range Correlations in Light Nuclei, Ph.D. thesis, Massachusetts Institute of Technology (2020).

[31] R. D. McKeown, J. Phys. Conf. Ser. 312, 032014 (2011).

[32] J. Alcorn, B. D. Anderson, K. A. Aniol, J. R. Annand, L. Auerbach, J. Arrington, 
T. Averett, F. T. Baker, M. Baylac, E. J. Beise, J. Berthot, P. Y. Bertin, W. Bertozzi, L. Bimbot, T. Black, W. U. Boeglin, D. V. Boykin, E. J. Brash, V. Breton, H. Breuer, P. Brindza, D. Brown, E. Burtin, J. R. Calarco, L. S. Cardman, R. Carr, G. D. Cates, C. Cavata, Z. Chai, C. C. Chang, N. S. Chant, J. P. Chen, S. Choi, E. Chudakov, S. Churchwell, M. Coman, E. Cisbani, S. Colilli, N. Colombel, R. Crateri, D. S. Dale, N. Degrande, C. W. de Jager, R. de Leo, A. Deur, G. Dezern, B. Diederich, S. Dieterich, R. di Salvo, P. Djawotho, J. Domingo, J. E. Ducret, D. Dutta, K. Egiyan, M. B. Epstein, S. Escoffier, S. Esp, L. A. Ewell, J. M. Finn, K. G. Fissum, E. Folts, H. Fonvieille, B. Frois, S. Frullani, H. Gao, J. Gao, F. Garibaldi, A. Gasparian, A. Gavalya, O. Gayou, S. Gilad, R. Gilman, F. Giuliani, A. Glamazdin, C. Glashausser, J. Gomez, V. Gorbenko, T. Gorringe, M. Gricia, K. Griffioen, D. Hamilton, J. O. Hansen, F. W. Hersman, D. W. Higinbotham, R. Holmes, H. Holmgren, M. Holtrop, N. D'Hose, E. Hovhannisyan, C. Howell, G. M. Huber, E. Hughes, C. E. Hyde-Wright, H. Ibrahim, S. Incerti, M. Iodice, R. Iommi, D. Ireland, S. Jaminion, J. Jardillier, S. Jensen, X. Jiang, C. E. Jones, M. K. Jones, K. Joo, C. Jutier, W. Kahl, S. Kato, A. T. Katramatou, J. J. Kelly, S. Kerhoas, A. Ketikyan, M. Khandaker, M. Khayat, K. Kino, I. Kominis, W. Korsch, S. Kox, K. Kramer, K. S. Kumar, G. Kumbartzki, M. Kuss, L. Lagamba, G. Laveissière, A. Leone, J. J. LeRose, F. Marie, L. Levchuk, M. Leuschner, D. Lhuillier, M. Liang, K. Livingston, R. A. Lindgren, N. Liyanage, G. J. Lolos, R. W. Lourie, M. Lucentini, R. Madey, K. Maeda, S. Malov, D. M. Manley, D. J. Margaziotis, P. Markowitz, J. Marroncle, J. Martine, S. Mayilyan, J. S. McCarthy, K. McCormick, J. Mclntyre, R. D. McKeown, D. Meekins, R. L. van der Meer, Z. E. Meziani, R. Michaels, B. Milbrath, J. A. Miller, W. Miller, J. Mitchell, J. Mougey, S. Nanda, A. Nathan, D. Neyret, E. A. Offermann, Z. Papandreou, C. F. Perdrisat, R. Perrino, G. G. Petratos, A. Petrosyan, 
L. Pierangeli, S. Platchkov, R. Pomatsalyuk, D. Pripstein, D. L. Prout, V. A. Punjabi, T. Pussieux, G. Quéméner, R. D. Ransomez, O. Ravel, B. Reitz, Y. Roblin, R. Roche, M. Roedelbronn, O. A. Rondon-Aramayo, P. G. Roos, G. Rosner, D. Rowntree, G. A. Rutledge, P. M. Rutt, M. Rvachev, F. Sabatavenere, A. Saha, T. Saito, F. Santavenere, A. J. Sarty, W. J. Schneider, J. P. Segal, A. Serdarevic-Offermann, A. Shahinyan, K. Slifer, T. P. Smith, A. Soldi, P. Sorokin, P. Souder, S. L. Spiegel, M. A. Stevens, S. Strauch, R. Suleiman, J. A. Templon, T. Terasawa, L. Todor, H. Tsubota, H. Ueno, P. E. Ulmer, G. M. Urciuoli, L. van Hoorebeke, R. van de Vyver, S. van Verst, P. Vernin, B. Vlahovic, H. Voskanyan, E. Voutier, R. Walter, J. W. Watson, D. P. Watts, L. B. Weinstein, K. Wijesooriya, B. Wojtsekhowski, H. Xiang, F. Xiong, W. Xu, D. G. Zainea, V. Zeps, J. Zhao, X. Zheng, Z. L. Zhou, L. Zhu, and P. A. Zolnierczuk, Nucl. Instruments Methods Phys. Res. Sect. A Accel. Spectrometers, Detect. Assoc. Equip. $\mathbf{5 2 2}, 294(2004)$.

[33] L. Ou, Precision Measurements of Electron-Proton Elastic Scattering Cross Sections at Large Q2, Ph.D. thesis, Massachusetts Institute of Technology (2019).

[34] D. W. Higinbotham, "Determination of the Beam Energy (ARC Energy \& Spin Precession)," DVCS Collaboration Meeting (2017).

[35] M. Mihovilovic, Measurement of double polarized asymmetries in quasi-elastic processes $3 H e\left(e, e^{\prime} d\right)$ and $3 H\left(e, e^{\prime} p\right)$, Ph.D. thesis, UNIVERSITY OF LJUBLJANA (2012), arXiv:1208.0748 .

[36] K. B. Unser, 23, 266 (2008).

[37] J.-C. Denard, A. Saha, and G. Laveissiere, , 2326 (2002). 
[38] D. Mack, Hall A Beam Current Monitoring Infrastructure, Tech. Rep. (2015).

[39] S. N. Santiesteban, Unser Calibration Fall 2018, Tech. Rep.

[40] S. N. Santiesteban, BCM Calibration October 05 2018, Tech. Rep.

[41] T. Day, "Harps for Dummies," https://www.jlab.org/accel/beam_diag/harp/ harp_dummy.html

[42] J. Bane, BPM calibrations from May 3rd 2018, Tech. Rep.

[43] P. Zhu, K. Allada, T. Allison, T. Badman, A. Camsonne, J. P. Chen, M. Cummings, C. Gu, M. Huang, J. Liu, J. Musson, K. Slifer, V. Sulkosky, Y. Ye, J. Zhang, and R. Zielinski, Nucl. Instruments Methods Phys. Res. Sect. A Accel. Spectrometers, Detect. Assoc. Equip. 808, 1 (2016), arXiv:arXiv:1509.03510v1.

[44] T. Hague, Calibrating The Hall A Raster, Tech. Rep. (2019).

[45] R. Lindgren, A CDR for a New Scattering Chamber for Hall A, Tech. Rep. (2001).

[46] D. Meekins, Hall A Tritium Target Configuration for December 2017, Tech. Rep. (2018).

[47] H. Dai, M. Murphy, V. Pandey, D. Abrams, D. Nguyen, B. Aljawrneh, S. Alsalmi, A. M. Ankowski, J. Bane, S. Barcus, O. Benhar, V. Bellini, J. Bericic, D. Biswas, A. Camsonne, J. Castellanos, J.-P. Chen, M. E. Christy, K. Craycraft, R. Cruz-Torres, D. Day, S.-C. Dusa, E. Fuchey, T. Gautam, C. Giusti, J. Gomez, C. Gu, T. Hague, J.-O. Hansen, F. Hauenstein, D. W. Higinbotham, C. Hyde, C. M. Jen, C. Keppel, S. Li, R. Lindgren, H. Liu, C. Mariani, R. E. McClellan, D. Meekins, R. Michaels, M. Mihovilovic, M. Nycz, L. Ou, B. Pandey, K. Park, G. Perera, A. J. R. Puckett, S. Širca, T. Su, L. Tang, Y. Tian, N. Ton, B. Wojtsekhowski, S. Wood, Z. Ye, and J. Zhang, Phys. Rev. C 98, 014617 (2018). 
[48] "Short Orbit Spectrometer in Hall C," https://www.jlab.org/Hall-C/equipment/ short\{_\}orbit.html ().

[49] "E12-11-112 Optics Run Plan," https://wiki.jlab.org/tegwiki/index.php/Fall_ 2018_Optics_Runs.

[50] K. G. Fissum, W. Bertozzi, J. P. Chen, D. Dale, H. C. Fenker, J. Gao, A. Gavalya, S. Gilad, C. R. Leathers, N. Liyanage, R. O. Michaels, E. A. Offermann, J. Segal, J. A. Templon, R. Wechsler, B. Wojtsekhowski, and J. Zhao, Nucl. Instruments Methods Phys. Res. Sect. A Accel. Spectrometers, Detect. Assoc. Equip. 474, 108 (2001).

[51] K. Allada, C. Hurlbut, L. Ou, B. Schmookler, A. Shahinyan, and B. Wojtsekhowski, Nucl. Instruments Methods Phys. Res. Sect. A Accel. Spectrometers, Detect. Assoc. Equip. 782, 87 (2015), arXiv:1502.01772.

[52] M. Iodice, E. Cisbani, S. Colilli, R. Crateri, S. Frullani, F. Garibaldi, F. Giuliani, M. Gricia, M. Lucentini, A. Mostarda, L. Pierangeli, F. Santavenere, G. Urciuoli, R. D. Leo, L. Lagamba, A. Leone, R. Perrino, S. Kerhoas, I. Lugol, B. Mazeav, P. Vernin, and A. Zaccarian, Nuclear Instruments and Methods in Physics Research Section A: Accelerators, Spectrometers, Detectors and Associated Equipment 411, 223 (1998).

[53] P. Solvignon, PID Detectror Calibration for E01-012, Tech. Rep. (2001).

[54] R. Brun and F. Rademakers, Nucl. Instruments Methods Phys. Res. Sect. A Accel. Spectrometers, Detect. Assoc. Equip. 389, 81 (1997).

[55] "Tritium Analysis Scripts," https://github.com/JeffersonLab/HallA-Online-Tritium.

[56] Y. Qiang, Hall A HRS and Septum Optics Database Calibration, Tech. Rep. (2003). 
[57] N. Liyanage, Optics Calibration of the Hall A High Resolution Spectrometers using the new optimizer, Tech. Rep. (2002).

[58] C. Gu, Spectrometer Optics Study for E08-027, Tech. Rep. (University of Virginia, 2016).

[59] C. Grupen and B. Shwartz, Particle Detectors (Cambridge University Press, Cambridge, 2008).

[60] R. C. Torres, Energy loss corrections and variable offsets using hydrogen data, Tech. Rep. (2018).

[61] K. Olive, Chinese Phys. C 38, 090001 (2014).

[62] S. Covrig Dusa, private communication.

[63] S. Li, Test Gas Target Density for Time-dependence, Tech. Rep. (2018).

[64] S. Santiesteban, S. Alsalmi, D. Meekins, C. A. Gayoso, J. Bane, S. Barcus, J. Campbell, J. Castellanos, R. Cruz-Torres, H. Dai, T. Hague, F. Hauenstein, D. Higinbotham, R. Holt, T. Kutz, S. Li, H. Liu, R. McClellan, M. Nycz, D. Nguyen, B. Pandey, V. Pandey, A. Schmidt, T. Su, and Z. Ye, Nucl. Instruments Methods Phys. Res. Sect. A Accel. Spectrometers, Detect. Assoc. Equip. (2019), 10.1016/J.NIMA.2019.06.025.

[65] D. Meekins, private communication.

[66] "Hall A Single Arm MonteCarlo for Inclusive Analysis," https://github.com/ JeffersonLab/halla-xem-analysis ().

[67] M. Berz and K. Makino, "COSY INFINITY version 8.1 user's guide and reference manual," (2001). 
[68] P. Vernin, J. Le Bars, H. Fonvieille, G. Quéméner, J. J. LeRose, J. Hogan, B. Vlahovic, and J. Billan, Nucl. Instruments Methods Phys. Res. Sect. A Accel. Spectrometers, Detect. Assoc. Equip. 449, 505 (2000)

[69] Zhihong Ye, "XEMC: Quasielastic Cross Section Model," https://userweb.jlab. org/ yez/XEMC/.

[70] M. E. Christy and P. E. Bosted, Phys. Rev. C 81, 055213 (2010).

[71] L. W. MO and Y. S. TSAI, Rev. Mod. Phys. 41, 205 (1969).

[72] N. Fomin, Inclusive electron scattering from nuclei in the quasielastic region at large momentum transfer, Ph.D. thesis, University of Virginia (2008), arXiv:0812.2144.

[73] P. E. Ulmer, "MCEEP: Monte Carlo for (e,e'p) experiments," https://hallaweb. jlab.org/software/mceep/mceep.html.

[74] R. Wiringa, "Two-Nucleon Momentum Distributions," https://www.phy.anl.gov/ theory/research/momenta2/

[75] M. Sargsian, private communication.

[76] S. Pastore, private communication.

[77] N. Santiesteban, private communication (). 